\title{
FROM
}

NEBULA TO NEBULA
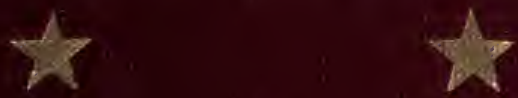

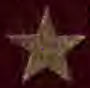

GEORGE HENRY LEPPER 


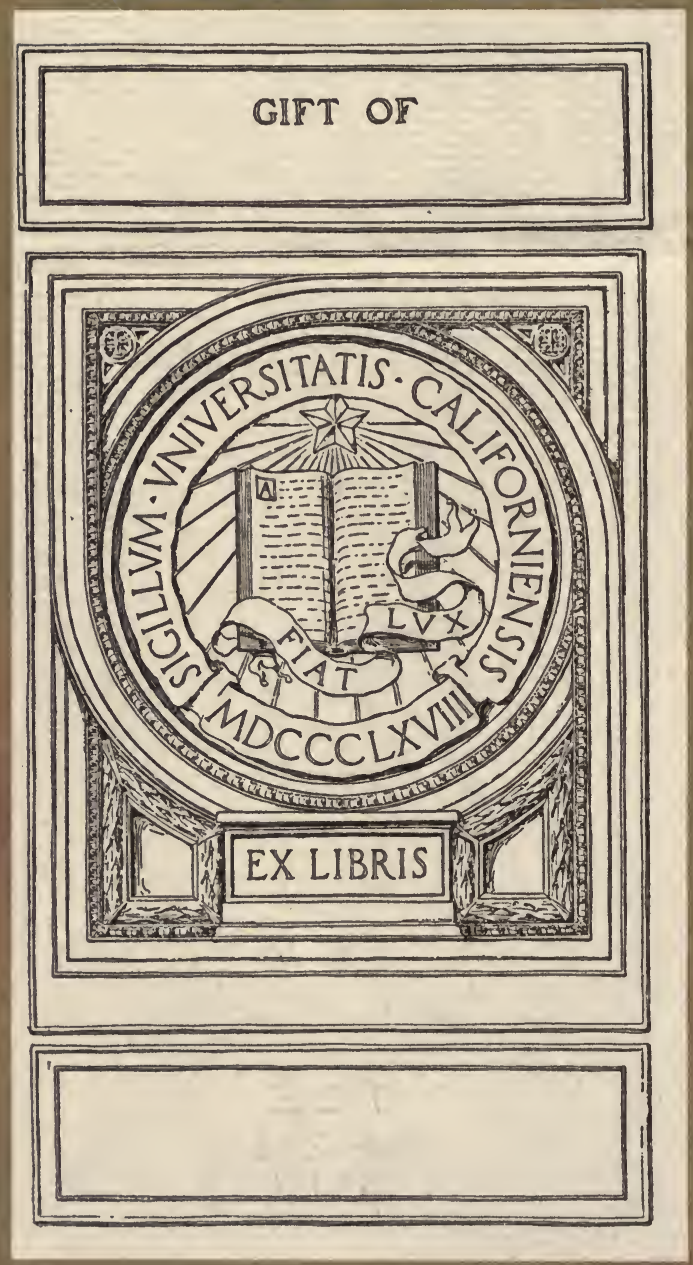




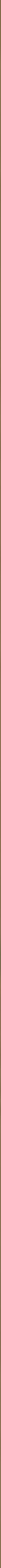


$100 x=0$

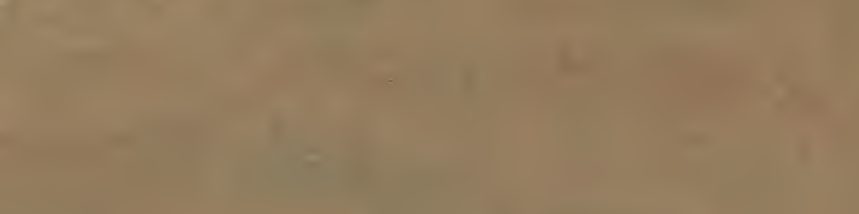

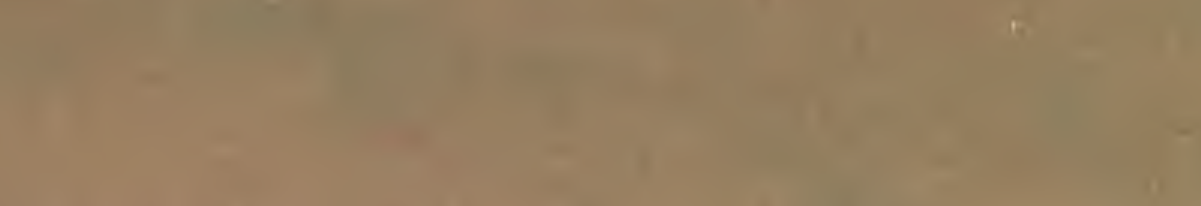

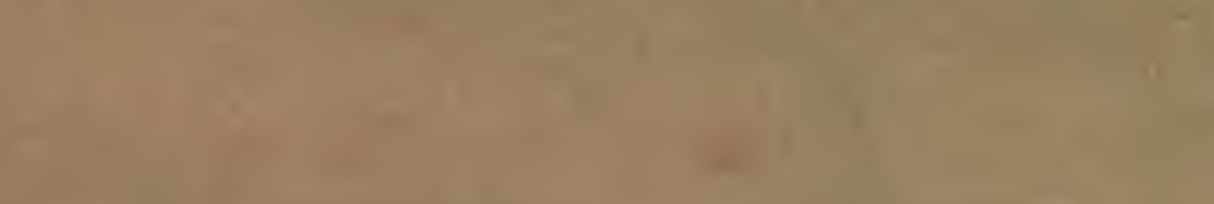

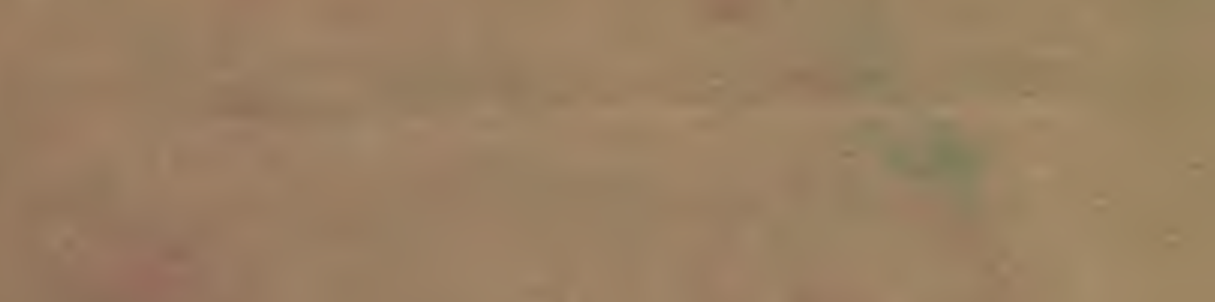

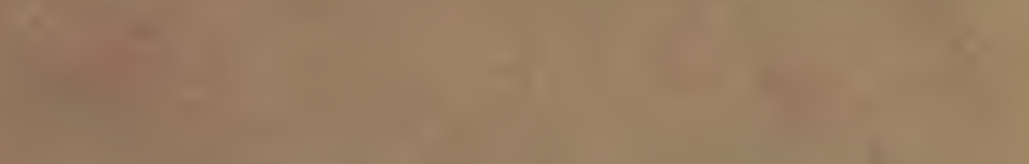
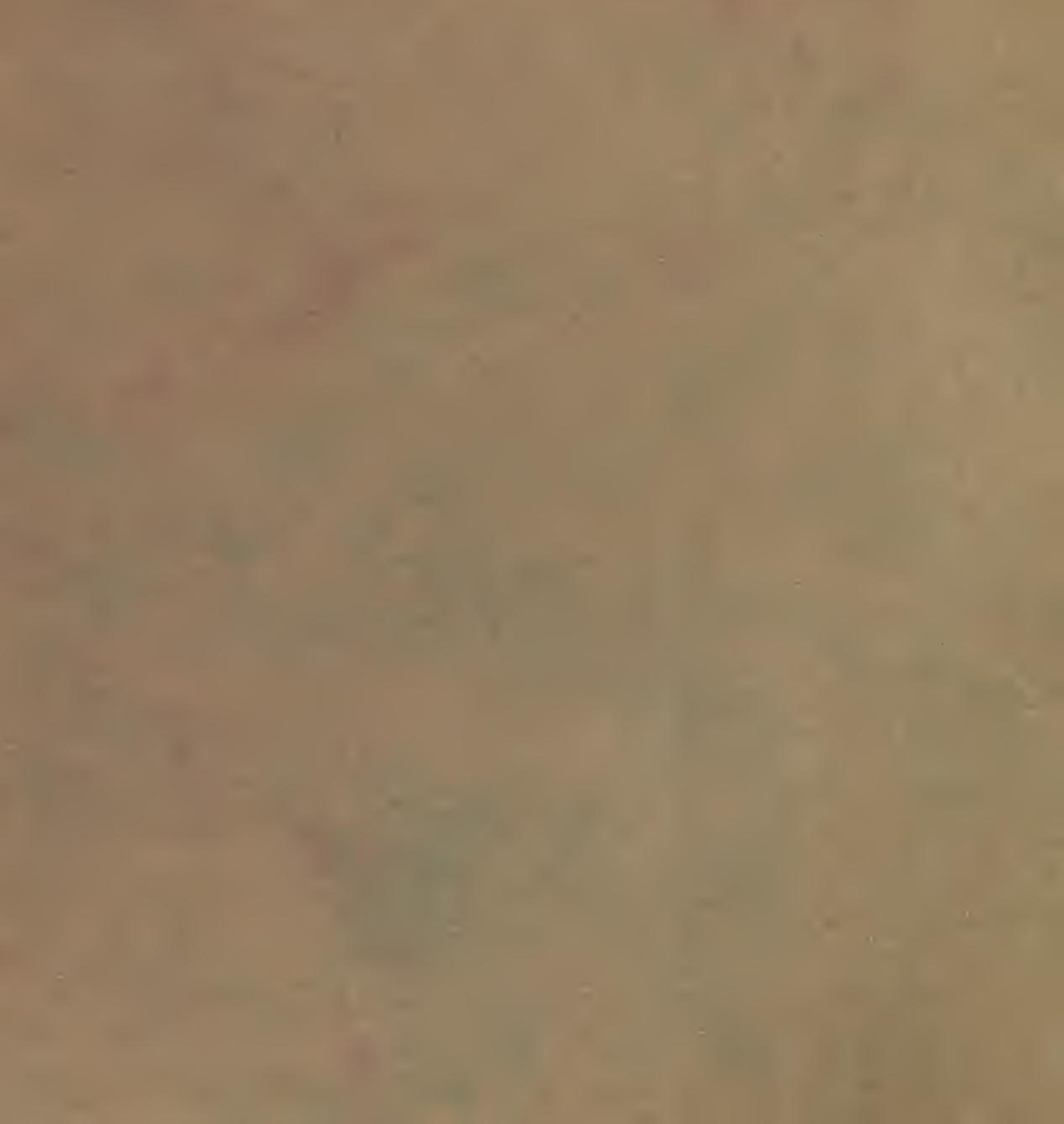




\section{$19-643 \%$}

\section{FROM}

\section{NEBULA ㄲo NEBULA \\ OR}

\section{The Dynamics of the Heavens}

\section{Containing}

A BROAD OUTLINE OF THE HISTORY OF ASTRONOMY A GENERAL SUMMARY OF ITS ACHUEVEMENTS

A SYNOPSIS AND CRITICISM OF RECENT COSMOLOGICAL, THEORIES

AND

espertally

AN EXPANSION OF THE PRINOIPLE OF UNIVERSAT GRAVITATION TO THE SOLUTION OF MANY OOSMIC PROBLEMS HERETOFORE DEEMEO INSOLUBLE

BY

\section{GEORGE HENRY LEPPER} MEMBER OF THE PITTSBURGH BAR

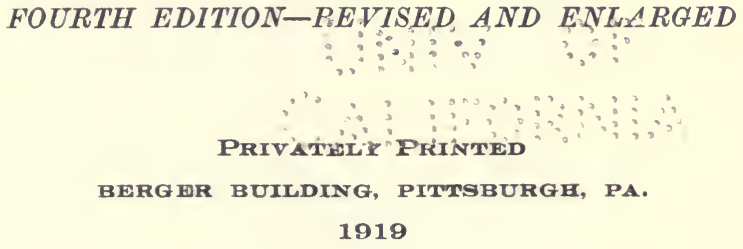




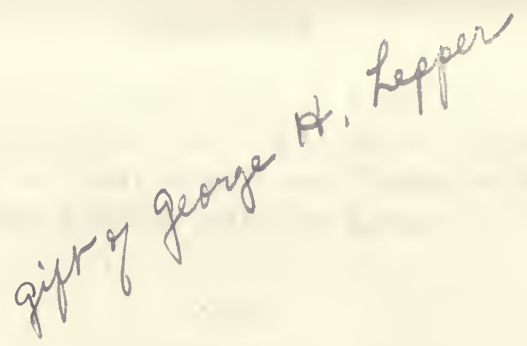

Copyright, 1912, 1913, 1917, 1919,

By George Henry Lepper

All Rights Reserved

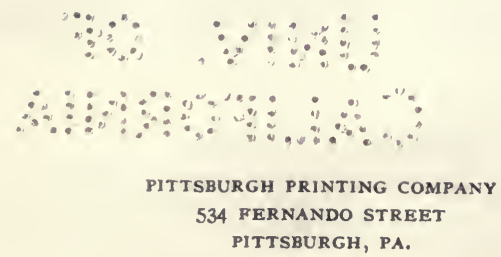




\section{CONTENTS}

Chapter

Page

I Introduction . ....................... 1

II The Author's Theories Outlined ......... 30

III Newton's Theory of Planetary Motions . ... 66

IV The Prime Resultant ................. 99

V The Law of Equilibrium ................. 128

VI The Tides $\ldots \ldots \ldots \ldots \ldots \ldots \ldots \ldots \ldots \ldots \ldots$

VII The Author's Theory of the Tides ......... 184

VIII The Nebular Hypothesis . . . . . . . . . . . . . . 213

IX Recent Cosmogonies ...................231

X The Sun $\ldots \ldots \ldots \ldots \ldots \ldots \ldots \ldots \ldots \ldots$

XI The Stars and Nebulex ..................293

XII Gravistatic Heat ......................... 314

XIII The Planets Mars and Venus ..............345

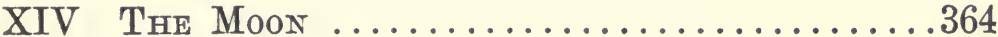





\section{INTRODUCTION}

THE object of this work is twofold: (1) to present a re-valuation of the time-honored doctrines upon 1 which modern theoretical astronomy is based, and (2) having shown wherein they are defective, to propose a new and far more comprehensive system revealing the entire visible universe in the philosophic aspect of a single organic unit co-ordinated throughout, as a priori it must be, by a single dynamical force. Like that of Newton, my system is founded upon the great underlying principle of universal gravitation, but it improves upon his, as a universe of three dimensions excels one of two, by extending this universality to include the clearly indispensable, yet hitherto strangely omitted, factor of the cumulative attraction of the stars and defining its wonderful functioning in the affairs, not only of our solar system, but of the stellar system as well.

During the two centuries which have elapsed since the publication of The Principia many important astronomical truths have come to light-the journey of the sun in space, the proper motions of the stars, star streams, the chemical constitution of the stars, the secular acceleration of the moon, the anomalous progression of Mercury's perihelion, the stupendous age of our earth, the existence and peculiarities of nebulæ, etc.-whose 
successful incorporation into gravitational astronomy demands not adaptation merely, but radical reconstruction.

By some strange spell our modern men of science have apparently succeeded in lulling themselves into the flattering notion that the astronomy they teach is an "exact" science and that all its really fundamental problems have been successfully disposed of. The truth is that not one of these great problems has been even approximately solved. The reason for this deplorable condition clearly lies in the failure of astronomers to have taken into account the greatest dynamical factor in the universe, namely, the mutual attraction between our solar system on the one hand and the rest of the universe on the other. Indeed, this factor, though occasionally alluded to in works on astronomy, is seemingly shunned as an impediment to astronomical progress; whereas, as I shall show, it is the one great indispensable agent required for the clearing up of all the myriad problems that concern planetary and stellar motions. Daring as Newton was to the eyes of his own generation in postulating gravitation as extending from the earth to the moon and from the sun to Saturn, he was yet not daring enough when he failed to extend the principle of universal gravitation to include the "fixed" stars. Nor have his followers repaired his error of omission. He construed our solar system as a universe unto itself utterly independent of and unaffected by the stars in general, the sun as stationary in space, and the orbits of the planets and the satellites as, literally, closed curves. In fine, he and his followers have been all along attempting to crowd into a grotesque $b i$-dimensional world, dynamically speaking, a real universe posessing three gravitational dimensions.

Before we proceed to this constructive branch of our subject, however, let us first listen to the testimony of the Newtonians themselves as to what they have achieved, and to what extent failed, in their self-appointed task of solving these 


\section{PARAMOUNT ASTRONOMICAL PROBLEMS}

1. The Origin and Maintenance of Celestial Motions.

2. The Law and Principle of Universal Gravitation.

3. The Tides.

4. The Comets.

5. The Source of the Sun's Heat.

6. The Individual Characteristics of the Planets and the Moon.

7. Stellar Problems in General.

8. The Genesis of the Solar System.

9. The Destiny of the Universe.

\section{Celestial Motions}

Newton, in common with his backward age, believed implicitly in the literal accuracy of the Mosaic cosmogony, namely, that only six millenniums before him Jehovah had created the heavens and the earth and, as part of the creative plan, launched the moon in her orbit around the earth and the planets in their orbits around the sun. By thus directly invoking divine intervention he evaded his obligation as a true scientist of assigning physical causes for what obviously are purely physical phenomena. Nor, as we shall later see, have his successors made good his delinquencies. The problem, however, was not simply that of getting the orbs into movement any which way, but to get them moving exactly tangentially, varying not by a single hairbreadth in a mile from mathematical accuracy, and adjusted to the strength of the central forces, in the matters of mass and velocity, with absolute precision. That Newton fully realized the demands he was thus making on teleological causes appears plainly enough from the following statement in Newton's own words as quoted by his biographer, Sir David Brewster(Memoirs of Newton, II. p. 81) :

To make such a system with all its motions, required a cause which understood and compared together the quantities of mat- 
ter in the several bodies of sun and planets, and the gravitating powers resulting from thence; the several distances of the primary planets from the sun and of the secondary ones from Saturn, Jupiter and the earth, and the velocities with which those planets could revolve about those quantities of matter in those central bodies; and to compare and adjust all these things together in so great variety of bodies, argues that cause to be not blind and fortuitous, but very well skilled in mechanics and geometry.

This enforced appeal of Newton's to supernatural agency to supply the chasms in his theory may have contented the two or three generations immediately succeeding him, but when a few score years later the new science of Geology had demonstrated the immense age of our planet, men's ideas of nature underwent a great philosophic change and they sought to escape altogether from the trammels of the Mosaic doctrine. Cutting themselves adrift, then, from the notion of a special creation, they attacked the old problems anew, as logically they were compelled to do. They asked themselves once more: How came the moon into her motion? How are we to explain the marvelously exact adjustments between the strength of gravity upon her on the one hand and her mass and velocity on the other? How came she, indeed, to be placed just where she is? What constitutes the "centrifugal force" by which she is enabled to react against the earth's gravity from year to year and age to age without sacrifice to her momentum?

It was just at this crisis that they should have bethought themselves of the one crucial factor that had not yet been considered by Newton; but if they thought of it at all, they probably spurned it as only an additional difficulty-of which they seemed then to have enough. That factor was the stellar resultant. Not knowing anything else to do, they resorted to shallow dogmatism: "Rectilinear motions", they decreed, "are self-existent and do not require explanation". This illuminating pronouncement runs current in all the so-called orthodox works on astronomy, and in corroboration I will quote here from two of them. The first excerpt is from the leading text-book on astronomy, by Doctor Charles 
Augustus Young, for many years professor of astronomy at Princeton University. He says (General Astronomy, Art. 400):

It has been customary with some writers to speak of a body thus moving "uniformly in a straight line" as actuated by a "projectile force", a very unfortunate expression, which is a survival of the Aristotelian idea that rest is more "natural" to matter than motion, and that when a body moves, some force must operate to keep it moving. The mere uniform rectilinear motion of a material mass in empty space implies no action of a physical cause, and demands explanation only as mere existence does. Change of motion, either in speed or direction-this alone implies force in operation.

The second is from the pen of that noted mathematician, Professor A. N. Whitehead (Introduction to Mathematics, p. 43) :

Galileo's successful experiment was not the result of a mere lucky guess. It arose from his correct ideas in connection with inertia and mass. The first law of motion, as following Newton we now enunciate it, is-Every body continues in its state of rest or of uniform motion in a straight line, except so far as it is compelled by impressed force to change that state. This law is more than a dry formula ; it is also a paean of triumph over defeated heretics. The point at issue can be understood by deleting from the law the phrase "or of uniform motion in a straight line". We there obtain what might be taken as the Aristotelian opposition formula: "Every body continues in its state of rest except so far as it is compelled by impressed force to change that state.

In this last -formula it is asserted that, apart from force, a body continues in a state of rest; and accordingly that, if a hody is moving, a force is required to sustain the motion; so that when the force ceases, the motion ceases. The true Newtonian law takes diametrically the opposite point of view. The state of a body unacted on by force is that of uniform motion in a straight line, and no external force or influence is to be looked for as the cause, or if you like to put it so, as the invariable accompaniment of this uniform rectilinear motion. Rest is merely a particular case of such motion, merely when the velocity is and remains zero. Thus, when a body is moving, we do not seek for any external influence except to explain changes in the rate of the velocity or changes in its direction. So long as the body is moving at the same rate and in the same direction there is no need to invoke the aid of any forces. 
To explain translatory motions by averring that they are "inexplicable" can scarcely be properly dignified as scientific or as a "paean of triumph". Granting, however, the possibility of such motions being inherent or self-existent, are we to understand that this is true of all bodies, or only of such as were primordial? If true of all bodies, it seems strange that Aristotle had not noticed it. On the other hand, if true only of primordial bodies, then must we not only class the sun and the planets as such, but asteroids and comets as well-which surely is absurd. Again, if celestial translatory motions are truly natural in the sense of being uncaused, then they might just as well have possessed velocities up to tens of thousands of miles a second and their directions have been equally diverse and erratic. To stipulate, $b y$ choice, the self-existence of such haphazard motions as these is only to begin with a chaos worse confounded and to render the task of Nature in the establishment of order all the more protracted and difficult.

And what of those marvelous adjustments subsisting throughout the universe between the centripetal forces on the one hand and the centrifugal on the other? How does it come that the earth, for example, is endowed with precisely the amount of velocity and physical momentum, and the exact and undeviating tangential motion, requisite to counterbalance the attraction of the sun? As to these adjustments our modern astronomers, less committal than Newton, maintain complete silence, leaving it to be inferred that the mere assumption of rectilinear motions carries with it, ipso facto, all the attributes with which we may find it expedient to endow them. They offer us nothing to take the place of Newton's "Cause not blind and fortuitous, but very well skilled in mechanics and geometry."

According to Newton's third law of motion, to every action there is always an equal and contrary reaction. It therefore follows that, inasmuch as the moon preserves her mean distance from the earth virtually unaltered from age to age, she is reacting against the latter's 
attraction with a force precisely equal thereto. But let me beseech you to compare these two forces of lunar action and reaction as defined by Newtonians to see whether they are in any sense equal. Granting to the moon an initial unearned momentum, that momentum is nevertheless a finite quantity which can neither be increased nor diminished of itself. If, therefore, it be ealled upon to react against an extraneous force, it must inevitably sacrifice some of its momentum, or, in other words, lose some of its velocity. On the other hand, the force it is pitted against, gravity, is a continuous force which, however long drawn upon, loses not an iota of its virtue. It can be shown that the attraction of the earth upon the moon at their present distance is equal to some $240,000,000$ million horse-power, for were she held for an instant motionless in her orbit and then let go, it would require that many horse-power to sustain her. In brief, Newtonianism has dared to marry, in undivorcible union, an imaginary, fortuitous, finite, unaugmentable and wasting force to that of centripetal gravitation, which in all things is precisely what the other is not. Astronomers are by no means oblivious to this absurdity (although they do not proclaim it from the housetops), as is shown by their studied avoidance of Newton's word "inertia" and the gratuitous substitution of the word "persistence" in its place.

Having now good-naturedly allowed ourselves to be persuaded by our Newtonian teachers that the reason why they have not succeeded in explaining translatory motions is no fault of theirs, but of the motions themselves in being inexplicable per se, that nice adjustments are so much matters of course that there is no need to discuss them, and that persistent and inertial are interchangeable terms, we look forward expectantly for a sweeping solution of all the riddles of the cosmos. Alas! only to be disappointed most grievously. Says Brewster, writing about the year $1850(M$. of $N$., II, p. 313) :

In concluding this brief notice of the progress of physical astronomy since the time of Newton in a few of its leading features, 
we are naturally led to ponder on the great truth of the stability and permanence of the solar system as demonstrated by the discoveries of Lagrange and Laplace. In the present day, when worlds and systems of worlds, when life physical and life intellectual are supposed to be the result of general law, it is interesting to study those conditions of the planetary system which are necessary to its stability, and to consider whether they appear to be the result of necessity or design. It follows, from the discoveries of Laplace, that there are three conditions essential to the stability and permanence of the solar system, namely, the motion of all the planets in the same direction,- -their motion in orbits slightly elliptical, or nearly circular,-and the commensurability of their periods of revolution. That these conditions are not necessary is very obvious. Any one of them may be supposed different from what it is, while the rest remained the same. The planets, like the comets, might have been launched in different directions, and moved in planes of various and great inclinations to the ecliptic. They might have been propelled with such varieties of tangential force as to have moved in orbits of great ellipticity; and no reason, even of the most hypothetical nature, can be assigned why their annual periods might not have been incommensurable. The arrangements, therefore, upon which the stability of the system depends, must have been the result of design, the contrivance of that omniscience that foresaw all that was future, and that infinite skill which knew how to provide for the permanence of His work.

\section{And a half century later Young says (G. A., p. 566) :}

In the present state of science many of the questions thus suggested seem to be hopelessly beyond the reach of investigation, while others appear like problems which time and patient work will solve, and others yet have already received clear and decided answers. In a general way it may be said that the condensation and aggregation of rarefied masses of matter under the force of gravitation; the conversion into heat of the (potential) "energy of position" destroyed by the process of condensation; the effect of this heat upon the contracting mass itself, and the radiation of energy into space and to surrounding bodies as waves of light and heat-these principles contain nearly all the explanations that can thus far be given of the present state of the he? venly bodies.

We see that our planetary system is not a mere uccidental aggregation of bodies. Masses of matter coming haphazard towards the sun would move as comets do, in orbits, always conic sections to be sure, but of every degree of eccentricity and inclination. There are a multitude of relations actually observed in the planetary system which are WHOLLY INDEPENDENT OF GRAVITATION AND DEMAND AN EXPLANATION. 
I. The orbits are all nearly circular.

2. They are all nearly in one plane (excepting the cases of some of the little asteroids).

3. The revolution of all is in the same direction.

4. There is a curiously regular progression of distance (expressed by Bode's law, which, however, breaks down at Neptune).

5. There is a roughly regular progression of density, increasing both ways from Saturn, the least dense of all the planets in the system.

As regards the planets themselves, we have:

6. The plane of the planet's rotation, nearly coinciding with that of the orbit (probably excepting Uranus).

7. The direction of the rotation the same as that of the orbital revolution (excepting probably Uranus and Neptune).

8. The plane of orbital revolution of the satellites coinciding nearly with that of the planet's rotation.

9. The direction of the satellite's revolution also coinciding with that of the planet's rotation.

I0. The largest planets rotate most swiftly.

In the first decade of the past century Laplace called the world's attention to these remarkable coincidences, and on the theory of probabilities demonstrated the chances of such an arrangement as now exists being fortuitous to be as many billions to one. At the same time he emphasized the utter helplessness of Newtonian theory to cope with these enigmas. All this he did as a prelude to the launching of his celebrated Nebular Hypothesis, the germ ideas of which were, (1) to seek for all the bodies in our solar system a common genesis, and hence concerted relations, and (2) to reduce to a modest single assumption of spontaneous motion the host of separate postulates theretofore so lavishly indulged in. All he asked of scientists was to concede him these two things: (1) incandescence for this nebula, and (2) that it possessed of itself, as something "natural" and "not requiring explanation", a rotation, round a fixed axis, as though it were a rigid solid. 
From time to time a few men have ventured to doubt the likelihood of so ethereal a thing as a nebula (a thousand times rarer than the air we breath) to rotate in this way, and have sought to assign mechanical causes by way of furthering the main purpose. Thus, in his First Principles (\$76) Herbert Spencer says:

If we assume the first stage in nebular condensation to be the precipitation into flocculi of denser matter previously diffused through a rarer medium (a supposition both physically justified and in harmony with certain astronomical observations), we shall find that nebular motion is interpretable in pursuance of the above general laws. Each portion of such vaporlike matter must begin to move toward the common center of gravity. The tractive forces which would of themselves carry it in a straight line to the center of gravity are opposed by the resistant forces of the medium through which it is drawn. The direction of movement must be resultant of these-a resultant which, in consequence of the unsymmetrical form of the flocculus, must be a curve directed, not to the center of gravity, but toward one side of it. And it may be readily shown that in an aggregation of such flocculi, severally thus moving, there must, by composition of forces, eventually result a rotation of the whole nebula in one direction.

Spencer's explanation, however, failed to satisfy his interpreter, John Fiske, who takes issue with him and in his magnum opus (Cosmic Philosophy, p. 360), adduces a version of his own, as follows:

Note first that we are obliged to accredit the various parts of this genetic nebula with motions bearing some reference to a common center of gravity; for the rotation of the resulting system must have had an equivalent amount of motion for its antecedent, and it is a well known theorem of mechanics that no system of bodies can acquire a primordial rotation merely from the interaction of its own parts. In making this assumption, however, we are simply carrying out the principle of the continuity of motion. It is not necessary to suppose, in addition, that all these motions primordially constituted a rotation of the whole mass in one direction. Such a hypothesis seems to me not only gratuitous, but highly improbable. It is more likely that these primeval motions took the shape of currents, now aiding and now opposing one another, and determined hither and thither according to local circumstances. In any case, such indefiniteness of movement must finally end in a definite rotation in one direction. For unless the currents tending eastward are exactly balanced 
by the currents tending westward-a supposition against which the chances are as infinity to one-the one set must eventually prevail over the other. And after some such manner as this our solar nebula must have acquired its definite rotation from west to east.

Why should we suppose, with Spencer, that the flocculi would fall only so far, instead of the whole way, and then obligingly take to rotating in accordance with Kepler's laws around a fixed axis; or why, with Fiske, that haphazard currents-proverbially fitful and inconstant-would forswear haphazardness and docilely $\mathrm{re}_{-}$ volve in ideally-ordered curves?

"But First Principles", the reader may interject, "was published in 1860, and Fiske's book in 1874; doubt. less something more definite and convincing has been forthcoming in the meantime." To satisfy the mind on this score I shall quote a late passage from the pen of Doctor T. J. J. See, the Director of the U. S. Naval O O servatory at Mare Island, California, and the author of the so-called Capture Theory (Art. Cosmogony, Americana) :

The solar system was formed from a spiral nebula, revolving and slowly coiling up under mechanical conditions which were essentially free from hydrostatic pressure. And spiral nebula themselves arise from the meeting or mere settling of unsymmetrical streams of cosmical dust. The whole system of particles has a sensible moment of momentum about some axis, and thus it begins to whirl about a central point, and gives rise to a vortex. In the actual universe the spiral nebulæ are to be counted by the million, and it is evident that they all arise from the automatic winding up of cosmical dust, under the attraction of their mutual gravitation. The two opposite branches of the spiral nebula, so often shown on photographs, represent the original streams of cosmic dust which are coiling up and forming gigantic spiral systems. *** The dust carried away (from the stars) by repulsive forces gathers here and there into clouds, and when such a mass settles the result is a cosmical vortex, which whirls and slowly develops into a cosmical system. Thus the stars by the expulsion of fine dust form nebulæ, and the nebulæ in turn, by condensation, form stars and systems. The clusters, like the whirlpool nebulæ, have a tendency to spiral movement. The attendant bodies, however, are never thrown off, but captured and added on from without. The heavy bodies drift toward the 
centers of attraction already developed, while the fine dust alone is diffused and carried away under repulsive forces to form other nebluæ which will sometime condense into cosmical systems.

The assumption that rectilinear motions are inherent is made to do duty to explain the flight of the sun, and, indeed, of the so-called "proper motions" of stars in general. How with every star, by hypothesis, moving at random there can exist so concerted a phenomenon as "star streams" has, so far as I am aware, remained not only unexplained, but even gone unremarked as food for reflection. As for the cause of the sun's flight, this is so far beyond the powers of Newtonian theory to cope with that the idea has seemingly never suggested itself; and the sun's velocity and the apparent direction of his course-bare facts in themselves devoid of philosophical interest-are all that Newtonianism can show for generations of laborious effort in this direction. Attend to this cheerless statement, if you please, from Doctor William Wallace Campbell's recent work (Stellar Motions, p. 194) :

These are frequent and legitimate questions: Is the solar system moving in a simple orbit, such as a conic section? Will it eventually complete a circuit in this orbit and return to the part of its orbit where it is now? The idea of affirmative answers to these questions appears to be prevalent in the human mind. It is natural to think that we must be moving on a great curveperhaps closed like an ellipse, or open like a parabola-the centre of mass of the universe being in the curve's principal focus. The attraction which any individual star is exerting upon us is certainly slight, owing to its enormous distance, and the resultant attraction of all the stars may not be very much greater; for since we are believed to be somewhere near the centre of our stellar, system, the attractions of the stars in the various directions should nearly neutralize one another, in accordance with the principle that a body situated within a concentrically homogeneous sphere is effectively acted upon only by the gravitational matter nearer the centre of the sphere than itself. Even though we may be following a definite curve at the present time, there is, in my opinion, little doubt that we shall be prevented from continuing upon it indefinitely. In the course of our travels we should be carried, sooner or later, relatively close to some individual star whose attraction would be vastly more powerful than that of all 
the other stars combined. This would draw us more or less from our present curve and cause us to follow a different curve. At a later date our travels might carry us into the sphere of attraction of some other great sun which would send us away in a still different direction. Thus, the chances are, in my opinion, that our path would, in time, be made up of a succession of unrelated curves.

The results deduced above define the direction and speed of the solar motion along a straight line; and, as a single line does not fix the position of a plane, we are without knowledge as to the plane in which the solar system is moving. It is of great interest that the present line of motion lies nearly in the plane of the Milky Way, making in fact an angle of about $I 7^{\circ}$ with the central line of the Milky Way. We need not concern ourselves at present with the question of the plane of our orbit, for the curvature of our path is undoubtedly so slight that we may consider it as a straight line for many generations of astronomers to come. (The italics are mine)

Another riddle presented in celestial motions for which Newtonianism can furnish no solution concerns the nebulæ, especially those known as spiral. Observation of these has revealed the unexpected circumstance that the more advanced the evolutional process the faster the rotation; indicating quite plainly that some centrifugal force must be at work gradually accelerating the gyration. Now, as everyone knows, Newtonianism regards all centrifugal force as independent of and opposed to gravity, hence what we must here look for to explain this phenomenon is either an increase of some force antagonistic to gravity or a diminution in the strength of gravity (centripetal attraction) itself. But this is not the Newtonian method, it seems; for, as will appear from the following quotation from Professor J. C. Kapteyn, he thinks to explain the increased centrifugalization by blaming it on an augmentation of its rival, the central attraction!

The phenomenon of the increase of velocity with the evolutional stage of the stars must give rise to speculation as to its cause. The observational results contained in our table naturally lead us to conclude that the matter from which the stars originate must have little or no velocity. How is this possible under the influence of the combined attraction of the rest of the system? Is it not as if gravitation had no effect on the cosmical matter in 
its primordial state? If this be so, as soon as matter changes from this state to another in which gravity [i. e. centripetal attraction] begins to act, or to act freely, motion [i. e. centrifugalization] will arise, and it is evident, that, as a rule, the motion must be accelerated, at least during immense periods, so that the longer the period elapsed since the birth of the stars the greater must be their average velocity.

\section{The Law and Principle of Gravitation}

Scientific students have become so accustomed to the idea of gravitation acting as a bond between cosmic bodies that they appear to have lost sight of the fact that, after all, it is essentially only an hypothesis, and that therefore its demonstration must be unequivocal and precise, or, as the lawyers say, beyond a reasonable doubt. Newton recognized this clearly, as was evidenced by his laying aside his earlier computations because of a conflict between the then existing data and the result he sought to prove. By a piece of rare good fortune the fatal error was subsequently corrected by others, and on making a second trial the new term was found to fit the case.

Now, there exist to-day two just such discrepancies between the observed and the computed places of bodies in our system which haunt the mathematical astronomer. The first is known as the anomaly of Mercury's perihelion movement, and the other as the secular acceleration of the moon's mean motion. There have been numerous attempts made to solve these irregularities by more and more exhaustive analyses, with the everpresent purpose of wiping them out of existence; but to no avail. As blots on Newtonian theory they are indelible. A few more-recent attempts have been made to explain them away as actualities, but equally without success. In the meantime the law of the inverse square stands gravely impugned. Empiricism is not science.But to quote the testimony of the Newtonians themselves: The first excerpt is from Young (G. A. p. 301):

There remains one lunar irregularity among the multitude of lesser ones, which is of great interest theoretically, and is still a 
bone of contention among mathematical astronomers, namely, the secular acceleration of the moon's mean motion. It was found by Halley, early in the last century, by a comparison of ancient with modern eclipses, that the month is certainly shorter than it was in the days of Ptolemy, and that the shortening has been progressive, apparently going on continuously $\circ \circ \circ$ In 100 years the moon, according to the results of Laplace, gets in advance of its mean place about IO", and the advance increases with the square of the time!

In passing, mark the highly significant fact that this law of the moon's advance coincides with the law of falling bodies.

My next quotation is from the pen of Professor Charles Lane Poor (The Solar System, p. 169):

Le Verrier discovered a slight irregularity in the motion of Mercury, which for over a half a century has been a source of trouble to astronomers and has led Newcomb to question the exactitude of the lawe of gravitation. The perihelion of Mercury's orbit has a secular perturbation, or regular forward movement, amounting to 579." 16 per century. Taking into account the disturbing action of all the known bodies in the solar system, Newcomb shows the law of gravitation will account for only 537. "62; or the perihelion of Mercury moves forward along the plane of the orbit by some $4 \mathrm{I} . " 54$ per Century in a manner that cannot be accounted for. In his Astronomical Constants Newcomb dismisses many possible explanations of this anomalous motion, and after careful treatment discards them all as untenable. $\mathrm{He}$ shows that this motion cannot be due to erroneous determination of the masses of the various planets, nor to hitherto undiscovered planets. For a readjustment of the masses,or the introduction into the system of new bodies sufficiently large to explain the discrepancy, will introduce serious discordances into the motions of the other planets. He seems to accept as the most probable hypothesis, first propounded by Hall, that the gravitation of the sun is not exactly as the inverse square but that the exponent of the distance is 2.0000001574 , instead of 2 . He provisionally accepts this as a working theory and introduces it into the computation of his tables of planetary motion.

Here we are confronted with a statement that may well give pause to those who harbor the belief that current astronomy is an exact science. It is not the law of the inverse square that our astronomers employ in their computations, but that of the inverse 2.0000001574 
power! Desirable though it may be-nay, commendable-to introduce short-cut methods for the purpose of getting at the results, the issue here is not one of expediency, but of philosophic truth. Why the necessity of this decimal? To this question, crucial as it is, Newtonianism has no defense to offer but the familiar excuse of the shoolboy: "It's the only way I can get the answer in the book." Incidentally, please to note that Mercury's perihelion problem, like that of the lunar acceleration, must also in some fundamental manner be governed by the law of falling bodies, as is evidenced by the fact that it is the exponent that requires "doctoring" in order to attain the desired result.

But not only does modern astronomy, albeit regretfully, thus impugn the great law of gravitation, it even repudiates the integrity of the principle itself! It is a long and tangled chain of ratiocination by which this desperate conclusion has been arrived at, but it is consistently Newtonian throughout. The following passages are taken from the work of Professor Frederick Soddy, of Glasgow University (Matter and Energy, pp. 19 and 112):

Before the doctrine of its conservation was established, energy was mysterious and unaccountable in its comings and goings. To-day it is no longer a mystery. The unaccounted-for appearance or disappearance of a quantity of energy in any process, however complex, would rouse as much scientific interest as the mysterious appearance or disappearance of matter. When it appears it must come from somewhere, and when it disappears it must go somewhere. Gradually this Law of Conservation has supplied the physicist with an experimental test of reality in a changing universe. What appears and disappears mysteriously, giving no clue of its origin or destination, is outside of his province. To him it has no physical existence. What is conserved has physical existence, whether it is tangible and ponderable like matter, or intangible and imponderable like energy. Early writers, when they really meant what is now called energy, often used the term force; and the idea of force, as will later be discussed, has confused the issues and retarded the growth of science to an almost incalculable extent. Carlyle says, meaning energy-"Force, Force, everywhere Force; we ourselves a mysterious Force in the centre of that "There is not a leaf rotting on 
the highway but has Force in it: how else could it rot?" The very idea of Force is, however, what would be termed an anthropomorphism; that is to say, it ascribes the behavior of inanimate objects to causes derived from the behavior of human beings. We have come to associate the motion of matter with somebody or something pulling or pushing it. When one body is observed to move toward another, like a stone falling to the ground, it has been supposed that, although no agent is visible, something must be pulling it. What, however, is actually observed is a change of position of the body, which acquires at the same time motion or velocity. The observation is correctly expressed by saying that energy, before associated with the position of one body with reference to another (potential energy), has changed into energy of motion (kinetic energy). To suppose that the one body attracts or pulls the other with a certain "force" is to imagine a cause which, if it existed, would account for the effect. Forces are not conserved, they have no physical existence, but they still survive even in scientific parlance, mainly because of the poverty of the language, which hardly allows effects to be expressed without some causal inference.

An ingenious theory of gravitation was put forward a century ago which, though not accepted, is very suggestive, and illustrates the difference between what science would consider a real cause and one that is fictitious, like the "force of gravity."

\section{The Tides}

The only excuse for our having a tidal theory at all is, of course, to connect the pertinent phenomena by their causal relationships. It so happens that Newton's hypothesis, for that is all it could fairly have been resarded in his day, was based on a single known circumstance and was conceived many decades in advance of a detailed knowledge of the observational data by which alone it can be established or disproved. These data Inay now be regarded as practically complete, after many decades of arduous and painstaking research, but unfortunately for the hypothesis the result is unequivocally adverse. Two of the most eminent scientists of recent years, Lord Kelvin and Sir George H. Darwin, collaborated for many years on this subject and the results of their inquiries are embodied in the latter's work, The 
Tides, from which the following condemnatory paragraphs are culled:

The equilibrium theory is nearly as much wrong as possible in respect to the time of high water. In fact in many places it is nearly low water at the time the equilibrium theory predicts high water. It would seem then as if the tidal action of the moon was actually to repel the water instead of attracting it, and we are driven to ask whether this result can possibly be consistent with the theory of universal gravitation (p. I6I).

In fact, the evanescence of the diurnal inequality is not much closer to the truth than the large inequality predicted by the equilibrium theory; and both theories must be abandoned as satisfactory explanations of the true condition of affairs (p. 180).

It is interesting to reflect that our tides today depend even more on what occurred yesterday or the day before in the Southern Pacific and Indian oceans than on the direct action of the moon today* the problem is one of insoluble mystery (p. I88).

Here, again, are two more to the same purpose, from Young's work (G. A., pp. 403 and 307):

The establishment of a port is the mean interval between the time of high water at that port and the next preceding passage of the moon across that meridian. At New York, for instance, this establishment is $8 \mathrm{~h}$. $13 \mathrm{~m}$. although the actual interval varies about 22 minutes on each side of the mean at different times of the month (p. 403).

In fact the siatical theory [of tides] becomes utterly unsatisfactory in regard to what actually takes place, and it is necessary to depend almost entirely on the results of observation, using the theory merely as a guide in the discussion of the observations (p. 307).

Besides this direct and obviously conclusive method of testing the validity of the hypothesis in question, there is another, which was first propounded by the philosopher Kant. Taking Newton's hypothesis for granted, Kant showed to the satisfaction of all who have come after him that, under the terms of the theory, the constant friction of the tides must, as a dynamical necessity, be gradually retarding the velocity of the earth's axial rotation, and that our day must consequently be 
lengthening. All his proofs, however, were in the nature of deductive reasoning, so that it devolved upon his followers to determine the question by interrogating the physical fact. This they have marvelously well succeeded in doing by mathematical means based on the records of certain ancient eclipses; but again with disappointing results. Says Young (G. A., p. 105):

At present it can only be said that the change, if any has occurred since astronomy became accurate, has been too small to be detected. The day is certainly not longer or shorter by I-IOO of a second than in the days of Ptolemy, and probably has not changed by $I-1000$ of a second.

What, then, in veiw of these indubitable disproofs, do you say should be done with Newton's tidal theory? Should it not be jettisoned at once and all haste made to find a better?

\section{The Comets}

These objects remain as great a mystery to men of science of the twentieth century as to Newton and his generation. It is true that some new information has been gleaned from the spectra of comets, and also from a critical study of their orbital vagaries; but as to their origin and nature, and the causes of the peculiarities exhibited by their tails, practically nothing has yet been satisfactorily determined. Young sums it up by saying (Art. 737) : "We have little certain knowledge on the subject."

\section{The Source of the Sun's Heat}

Newton sought to explain the light and heat of the sun by supposing the original chaos to have been composed of two main kinds of matter, luminous and nonluminous, and then picturing the Creator as segregating the first kind into the central body that we call the sun, and molding the rest into the earth, moon and planets. A century later Laplace postulated incandescence for his nebula and thence derived, not only our glowing luminary but the planets, also, in an original state of fusion. 
Shortly before the middle of the past century the theory of the mechanical equivalent of heat had its birth, and it was then immediately contended that the Nebula must have been cold to start with, but that the kinetic energy developed by the falling together of its component matter into the large bodies of the sun and planets initially heated them in a degree proportional to their respective masses. Mathematicians, however, soon showed that, so far at least as the sun was concerned, the new hypothesis was absurdly below requirements; and this finding it was that evoked the suggestion from Helmholtz that the sun is still a contracting body, that is to say, one in which the energy due to "position" is still in process of conversion into kinetic energy. Although this theory was tenaciously adhered to for decades, in spite of its geological contradictions, it finally succumbed to the joint attack of the mathematician's pencil and the new champion, Radium. But alas, and alas!, according to Doctor S. A. Mitchell (Pop. Astr., June, 1913) :

From theoretical considerations we are positively convinced that there must be radium in the sun. But to prove this is another problem! With the spectra we already have, we can prove nothing but coincidences.

In a nutshell, then, the source of the sun's heat is still a wide-open question.

\section{Individual Characteristics of the Planets}

Given sound principles of cosmology, scientists ought to be able to deduce from the main facts of the individual case, i. e., the mass of the planet and its solar distance, just what its surface, climatic and other conditions must be; resorting to instrumental observation more as a check than for a solution. That the principles on which they rely are not sound, however, is demonstrated by the controversies now going on between the "Vulcanists" and "Bolidists" as to the character of the moon's surface, and between the "Canalists" and "antiCanalists" as to the meaning of the Martian lines, etc. Indeed, it will be found difficult to cite a single instance 
where astronomers have been able to anticipate, deductively, the revelations of the telescope, the spectroscope and the bolometer. Thus, Jupiter and Saturn were believed to be ice-cold until the telescope showed them to be hot; the Martian canals were a complete surprise, and until the first edition of this work appeared that planet was assumed to be arid, whereas he is now known to be well provided with oceans and clouds; the full moon was believed as a matter of course to be roasting hot, till Langley's bolometer proved it to be intensely frigid, and so on. Surely the aim of science is to interpret Nature by the exercise of our reason and intelligence, not to stare her out of countenance by main force.

\section{Stellar Problems}

Very much the same sort of criticism may be made respecting the present scientific methods of dealing with the problems presented by the stars. Nothing could excel the care, skill and industry with which astronomers have performed the drudgeries of observational investigation and mathematical computation, bringing virtually to a state of repletion the stock of ascertainable data wherewith to erect a sound and comprehensive uranography. Just here, however, is where their progress halts. The workmen have indeed provided and delivered to hand the building materials, but the architect has been wanting. What we want to know, and what astronomers cannot tell us, is, how this plethora of material is to be fitted together. We want to know, specifically, such things as these:

Is there a universe beyond our own? What is the meaning of the great aggregation of stars in the Milky Way? Why are so many millions of the stars contemporaneously brilliant? Is there any rule by which we ean determine the intrinsic sizes of stars? What is the origin of the nebulæ? Why do they rotate? What is the explanation of their diversity of form? Why do the velocities of stars vary according to their spectral class? Are the stars all alike in chemical composition? How do they, in 
spite of gravity, preserve themselves aloof from one another? Why do "maturer"' systems revolve more rapidly than those more youthful? Is there acentral sun? Is there a maximum size to which stars can attain? What is the explanation of star streams? Why is the radius of the celestial circle of precession the same as the inclination of the earth's axis? What produces the sun's flight in space? What are the elements of its orbit?

To all such questions, elementary though they are, contemporary science returns no answers save of admitted speculation.

\section{The Genesis of the Solar System}

For nearly three quarters of a century, and until a decade or two ago, the Nebular Hypothesis of Laplace was generaliy esteemed as the true exposition of the modus operandi pursued by Nature in the evolution of the solar system. Now it is no longer so regarded by the great majority of astronomers, and, more particularly, by those who have made this subject a special study. Although it is quite true that numerous other hypotheses have arisen to compete for its vacated place, the fall of the Hypothesis has not been owing so much to their successful rivalry as to the belated recognition of its own glaring defects. As a matter of fact, never since Newton has the scientific problem of the genesis of the solar system been in a more chaotic and discouraging state than at this very time, in spite of, or perhaps because of, the distracting multiplicity of rival cosmogonies.

\section{The Destiny of the Universe}

From the standpoint of general philosophy probably the chief cosmological query is as to the permanency or impermanency of the Present Order. According to the hierarchy of modern science, this order is not permanent, the great cosmic machine is "running down", and it is only a question of time till it shall destroy itself. College professors who have grown gray in the teaching of this 
vicious and altogether erroneous doctrine have doubtless become hardened to its monstrosity, for they seem to proclaim it in and out of season with every mark of proud paternity! For my part, I regard such a conclusion as, on the face of it, so gross a reductio ad absurdum as by itself to refute the entire process of ratiocination by which it has been reached, even if in its wake lay, one after another, complete solutions of all the paramount problems above considered, instead of the lengthening trail of incompetence and failure that really does mark it.

\section{In the words of Balfour Stewart:}

We are led to look to a beginning in which the particles of matter were in a diffuse chaotic state, but endowed with the power of gravitation, and we are led to look to an end in which the whole universe will be one equally heated inert mass, and from which everything like life or motion or beauty will have utterly gone away.

Recapitulating, then, we find our modern Argonauts of science starting out to explore and interpret the universe by, first of all, methodically casting overboard that heretofore faithful compass of mankind, the natural law of cause and effect; stigmatizing, as behind the times, those of us who continue to cling to the belief that mechanical effects, whether in the skies or in the laboratory, can derive existence only from preëxisting causes; decrying the notion that gravitation is an objective reality, and in its stead substituting the metaphysical abstraction of "position"; proclaiming by precept the law of the inverse square, and inconsistently employing an admittedly empirical formula in practice; setting up, one after another, and again and again, spurious hypotheses as to the origin of cosmic heat, seemingly for the mere sport of knocking them down; tendering us a job lot of cosmogonies from which to make choice, not one of which but flouts Nature and her laws; promulgating a theory of tides, then conclusively disproving it, and afterward, knowing its falsity, continuing nevertheless to teach it as true and as a "guide" to the observations; depending helplessly upon mechanical implements rather than upon 
the intelligence to interpret the planets; asseverating the random, haphazard and spontaneous nature of stellar motions, and in the same breath professing to be searching out the laws governing this randomness; and, finally, impugning the integrity of Nature and recklessly condemning her to ignominious death!

Here, then, is the net result of two centuries of Newtonian philosophy - of the labor of hundreds of thousands of individuals who, in one capacity or another, have bent themselves heart and soul to the task of solving these great problems, and of untold millions of treasure unstintingly poured out by philanthropists and governments to the same end! Who is there so blind as not to see that Newtonian science has reached the limit of its development? Like the Ptolemaic system, it is only an approximation to the ultimate truth, though, to be sure, a very much closer approximation. The epicycle system broke down of its own weight because there always remained, after each cycle superadded, a diminishing, yet still appreciable, discrepancy to be accounted for. Slight as this excess must have seemed at the last, its correction was destined never to be achieved by the then time-honored method, but only by a complete overturning of the system by Copernicus and Kepler. The full development of Newtonian theory has conducted us of this day to a strangely similar impasse. There is now that apparently trifling, but really crucial decimal of Newcomb's to be accounted for! Every effort of mathematics has been exerted to iron it out, but in vain ; and it is only fair to conclude that it is permanently beyond the power of prevailing theory to cope with. I expect to prove to the satisfaction of the reader that the only way possible to overcome this obstacle is, by a revolution of the present-day science of astronomy no less drastic than that from the Ptolemaic to the Copernican, a revolution, too, which will abundantly justify itself by automatically solving all the paramount problems above enumerated. 


\section{The Elusiveness of the Obvious}

'To my mind, the most striking mental peculiarity of mankind in the past has been its proneness, in all ages, to focus attention on the things at a distance rather than on those close at hand, to prefer the abstruse to the simple, the miraculous to the natural, the obscure to the obvious. In fact, the epochal events in the life of Astronomy have all been spectacular revelations of truths that ought to have been almost intuitively perceived. The conspicuous dises of the sun and moon, for instance, and the rounded outline of the earth's shadow thrown upon the latter in eclipses, are phenomena too plain, one would suppose, for even a thoughtful boy to misinterpret. How, again, men in their sanity could for fourteen centuries on end-as did the Ptolemaists-prefer to believe and to teach that the vast heavens rotate daily around our little grain of dust, instead of the latter turning upon its own axis, surpasses our modern understanding. It took fifteen centuries, from Ptolemy to Kepler, to evolve a mind capable of conceiving the simple notion that circulating bodies-the moon and planets-might turn around their primaries in another kind of curve than a circle, and it took even the great mind of Kepler a score of years of toilsome effort to gestate the thought. And yet, again, not until a paltry two centuries ago did a Newton arise to suggest and prove that gravitation extends beyond our atmosphere. It has been said of Bismarck that his success as a statesman largely lay in frankly avowing his true motives, because he had observed that openness in a diplomat was the last thing generally looked for, and hence was a better cloak than any form of deceit could be. The reason the obvious in Nature goes unrecognized so long is because artful man misdoubts her plain message and in a spirit of subtlety reads in a farrago of irrelevancies between the lines. Lovers of Dickens will recall Mr. Pickwick's momentous antiquarian discovery, on the premises of one Bill Stumps, of a stone bearing a curious legend out of which Mr. Pickwick drew some twenty-seven recondite meanings, when, as a matter of fact, according to the 
veracious graver himself, the inscription read, simply, "Bill Stumps, his mark". If Mr. Pickwick could come to "life" again, I fancy that he would find himself very much at home in the society of many modern astronomers.

A great orator has said that most men find it easy enough to believe in miracles having occurred two thousand or more years ago, or that they will again occur two thousand years hence, but that it is next to impossible to find anyone willing to credit their happening to-day. Paraphrasing this epigram, I would say of Newtonians, in general, that they show no compunction about postulating mechanical miracles as freely taking place far up in the firmament, or deep in past time, while they would indignantly scout the possibility of such things occurring right here and now. Thus, they nonchalantly predicate the spontaneous rotation of the Laplacian nebula, though they would summarily classify as incurably defective a child of six who would expect as much of a mere squirrelwheel.

\section{UnUm versus OMnes}

On the theory of probabilities, the odds in every generation are a billion to one against the originator of an idea being right and the world wrong. This formidable preponderance of numbers against him has doubtless awed many a timid, but correct, thinker into silence, and cast many a stone in the path of human progress. Few of us, I dare say, ever stop to reflect that every valuable idea and item of information has incipiently been handicapped by identically these odds, and that all that mankind has garnered, or can ever acquire, in the way of useful knowledge is merely the sum of these minority-of-one discoveries that have won their way through gauntlets of criticism to general acceptance. In every ship's company, at the close of every voyage, there is invariably one who must be the first to sight port. Natural truth exists independently of both human wishes and opinions, and cannot be settled one way or the other by a plebiscite, or even by a council of the wisest of one's own generation. It is re- 
lated of Newton, for instance, that, although he survived for forty years the publication of his great work, The Principia, he did not have upwards of twenty followers in all England at the time of his death, and that it was not until ten years later that his doctrines were allowed to be taught at Cambridge and Oxford in competition with those of Descartes! Truly, a prophet is often without honor in his own generation as well as in his own country. O tempora! O mores! To-day Newton's fame fills the world, and a new generation has arisen that honors his opinions above Nature's truths, and even counts it a virtue to shield his errors against exposure and correction!

The generality of mankind appear to labor under the fond delusion that in the serene domain of pure science are to be found all the noblest scientific virtues in their highest perfection-receptivity, open-mindedness, frankness, catholicity, intellectual hospitality, and the like. In view of the pitiful failures they have scored all along the line, and which I have just outlined, we should especially expect of Newtonians a generous share of that divine humility which their renowned leader displayer when he uttered those memorable words: "I know not what the world will think of my labors, but to myself it seems that I have been but as a child playing on the seashore; now finding some pebble rather more polished, and now some shell rather more agreeably variegated than another, while the immense ocean of truth extended itself unexplored before me." But contrast with this sentiment the following passage from the pen of the distinguished Director of the Smithsonian Astrophysical Observatory at Washington, Doctor Charles G. Abbot. In his learned work, The Sun (p. 8), he says:

Every large scientific institution or observatory has almost daily communications from persons of very moderate attainments who presume to question, nay rather to spurn, the most well-attested facts of human knowledge. Such persons seem to prefer especially to direct their attacks on the following facts: the Copernican system; the law of universal gravitation; the first and second laws of energy; and, finally, the high temperature of the 
sun. No argument can refute them, because they have not the requisite learning to comprehend it, which is no disgrace, but which should make men modest enough to have faith in those who excel them immeasurably. Hence it is the policy of most scientific institutions to avoid entirely discussions of these subjects with such correspondents.

Since the initial publication of this work, in March, 1912, many of the ideas therein for the first time presented to the world have reappeared in the public prints, but always fugitively, in strange uniforms, and impressed into the service of alien doctrines against which I have taken up arms. This state of affairs leads me to emphasize the truism that a system of philosophy or science, such even as my own, like an automobile or any other complicated mechanical contrivance, is useful and valuable only as an assembled whole. What I have aimed to do, and what I venture to believe I have succeeded in accomplishing is, in Emerson's pregnant phrase, to reveal the universe "as a transparent law, not a mass of facts"; or, in concreter terms, as an eternal, self-energizing, selfregulating entity, coördinated throughout by a single (necessarily single) underlying dynamical principleGRAVITATION. Some, indeed, may say that I have cast my net too wide; that I have presumptuously attempted to solve too many problems at one stroke. I myself do not think so ; not, indeed, that I presume to insinuate that my offered solutions are conclusive and final, but because the very nature of my undertaking involves comprehensive treatment. A globe-map of the earth, however slightly filled in, is quite as essential to a correct knowledge of geography as a full complement of scattered maps of detail. In this book I seek to compress the universe about us into a single concept, a sort of mental universe-map, as it were, in which the sum of creation may be apprehended as the automatic unit that our intelligence tells us it must be in order to exhibit the infinite harmonies it does.

The process of building up a science such as astronomy has often been likened to that of erecting a great sathedral. The resemblance is palpable enough, but there is a contrast which here more deserves our notice. In the 
case of the cathedral, the design in the mind of the architect comes first in order of time, following which, bricks, stones and timber are all manufactured, cut and fashioned to fit their predestined places, and then respectively delivered upon the premises systematically so as to forestall possiblemistakes and confusion. Moreover, the architect takes the builder into his counsels, supplies him with duplicate plans and specifications, and in every other way coöperates with him throughout. In the case of constructing a science, all this is reversed. The designer never appears; there are no plans or specifications; facts - the building materials-are acquired haphazard and at awkward intervals; the very size of the foundation is unknown, as are likewise the height, the breadth and the general arrangement of the superstructure. Taking facts as they arrived, astronomers have tried to make progress as they went along, often mistaking capstone for cornerstone, cornice for girder, lintel for sill-in short, creating a structure, it is true, but one weirdly different from what it would have been had all the materials been available from the very outset. Now, thanks to time and human industry, all the facts are simultaneously before our eyes inviting and challenging us to test our architectural powers afresh. Shall we decline the challenge? 


\section{THE AUTHOR'S THEORIES OUTLINED}

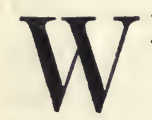

HETHER the universe is self-existent, selfcreated or the work of Omnipotence is not a problem for the scientist as such. His province is strictly limited, by choice as well as by definition, to the study of matter in its various forms, organic and inorganic, animate and inanimate, and the tracing out of the operations of the natural law of cause and effect as far as he is able. Of all men, the astronomer should rightfully be expected to be the most uncompromisingly scientific, inasmuch as his work deals with matter in the mass entirely uncomplicated by such things as chemical or biological activities.

Fortunately for the validity of our scientific conclusions, it is all one whether we regard the universe as self-existent or God-created. For if self-existent we have no choice but to accept the Present Order as the inevitable outgrowth of a past eternity of mutual accommodation of parts under the guidance of natural law, while if we regard it as the handiwork of Omniscience we thereby philosophically concede both its original and present perfection, and hence its insusceptibility of correction or improvement. In fine, the Present Order must be logically accepted as perpetual. Here we come upon one of the main points of difference between the accepted teaching and my own; for whereas I affirm the perpetual integrity of the universe, the world of science flatly denies it. 
Another preliminary question to consider is this: Is the universe finite or infinite in extent? According to the doctrine of the Conservation of Energy, the universe as a machine is "running down". We are taught that, precisely as matter in the "Beginning" existed of itself, so did its motions, and, further, that this original energy of motion has ever since been undergoing transmutation into thermal energy by the agglomeration of this matter into larger and larger, and by the same token into fewer and fewer, units. This process, they go on, is to continue indefinitely until every vestige of the primordial cosmic dust shall have been swallowed up in one final immense body, which by slow contraction will become solidly compact, dissipate all its heat into surrounding space, and eventually deteriorate into nothing less inglorious than a monstrous junk-heap of perished worlds. The only condition on which they will concede the universe to be eternal is that it be veritably infinite in extent, so that this procession of stars plunging to their common doom may go on interminably.

Against this whole conception I protest most vehemently; holding that, whether the universe be finite or infinite in extent, the Present Order is potentially permanent and enduring. I contend, with Aristotle, that rest is the natural state of matter, from which every departure must be susceptible of dynamical explanation; and in defense of this conviction I expect to show to the satisfaction of every open-minded reader that the so-called inherent motions of stars, planets, sun and nebulæ are legitimate gravitational phenomena easily explicable by the aid of the stellar resultant. Reason as we will, however, about gravitation, it will always remain in essence a force of concentration, of centralization; and if not efficiently counteracted it spells universal ruin in the end, however long deferred. Is there, then, no counterpoise to this of Nature's shrewd devising? Has not her duality, her trick of pairing opposites, long been the fertile theme of philosophers and scientists, and shall her ingenuity fail her in this, her 
direst need? Let us, then, consider her cosmic processes under these two heads:

1. The Principle of Concentration, or Attraction.

2. The Principle of Dispersion, or Explosion.

\section{THE PRINCIPLE OF CONCENTRATION OR}

\section{ATTRACTION}

When Newton conjured with that magical phrase, universal gravitation, he little realized how much more it meant than he construed it to mean. For ho seems never to have thought of extending the principle to the stars, but inconsistently restricted it to the solar system, which we all know is little more than a molecule in the greater cosmos. Accordingly, he assumed the sun to be stationary in space and the orbits of the moon and planets to be, literally, closed curves; quite as though they kept threading the same tunnels in the stagnant ether forever and ever. In short, he conceived our system to be a universe unto itself wholly independent of and uncorrelated with the stars in general. It is now known with absolute certainty that the sun is flying through space with a velocity of some ten miles a second, and that the orbits of the planets are far from being the re-entering curves that Newton thought them. Here it lay upon the surface that a discovery so amazing might well be fraught with far-reaching potentialities for extending our inquiry beyond the confines of our system into that of the stellar universe beyond. Unfortunately, however, with their customary genius for missing the obvious, instead of asking themselves here whether the cumulative gravitation of the stars might not be the cause for this new phenomenon, our Newtonian friends contented themselves with merely rehashing the old banality by saying that such motion is "natural" with the sun, as with all other cosmic bodies, and "demands explanation only as mere existence does". As for the planets sharing this same motion of translation, they 
say it is "because the sun pulls them with him by main force, that of his attraction".

It seems to me self-evident that our Present Order could never have existed as long as it manifestly has, or that it can continue to exist beyond to-morrow, without the presence of some single, all-embracing, co-ordinating dynamical principle underlying the whole cosmos. In casting about for this principle, where shall we find one so eligible or readier to hand than that of universal gravitation itself, with the emphasis on the adjective? Let this, then, be our line of inquiry: What effect, if any, has the factor of the mutual attraction between the solar system, on the one hand, and the remainder of the universe, on the other, upon the conduct of the former?

\section{Celestial Motions}

In the outset let me say that I shall not undertake to discuss the essence of gravitation, or to explain how it acts across the void. Let these for the present be taken for granted. All I desire now to interpolate in this connection is, that it is no greater tax upon our powers of comprehension to understand how gravitation can extend from the earth to a star than how it manages to span the blank between the earth and a balloon, or between the sun and Neptune. The only way to settle the question is to put our hypothesis to the test. If gravitation does not extend from the earth to the stars, the phrase universal gravitation is a misnomer. On the other hand, if it does so extend, the present theory of astronomy, which leaves the factor of the stellar attractions entirely out of the reckoning, cannot possibly be final. This work, so far as I am aware, constitutes the very first attempt to repair this grave omission.

The first important point to consider is the nature and composition of this resultant of the stellar attractions. Pick out one particular star and consider it separately with reference to our earth. Obviously, a mathematically straight line connecting the center of mass of the earth with the center of mass of the star will 
indicate the line of attraction between the two bodies; this attraction, of course, varying directly as the product of their respective masses and inversely as the square of their separating distance. As a consequence, the earth is impelled to fall toward the star, which in turn falls earthward. Now, a.falling body, unlike any other sort of motion we know of, "advances with the square of the time", a peculiarity which, as we shall presently see, supplies us with a very valuable corroboration of our main argument.

Now pick out a second star, a third, a fourth, a twentieth, in divers sections of the heavens and repeat the performance made with the first, and then try to imagine as well as you can (as by picturing them luminous) the net work of rays from all the stars simultaneously focussed on our planet. What, think you, should be their cumulative effect upon the ball of the earth? Should not this latter fall in the direction of the maximum pull? Does it not also seem natural that it should so fall with its center of gravity at the lowest point, that is, with its heavier hemisphere underneath? Should not so many divergent stresses, all acting in unison, work a torsion within the globe and incline it to gyrate upon its axis?

Turning now to contemplate the earth directly, we perceive at once that her axis is "fixed", which is just what we should look for under our hypothesis; and this fixation we note persists in spite of the fact that she revolves yearly around the sun. Secondly, we observe a very marked difference between our northern and southern hemispheres; the former comprising nearly two and a half times as much land as the latter, while a closer inspection discloses the continents so eagerly seeking the north pole as to seem to be shouldering each other away from it, like cakes of ice jammed by the current against a bridge pier. Thirdly, there is the $o b$ lateness of the earth's figure, which tells as plainly as words that the globe is settling upon itself, like a ball of soft putty, and that its present shape is but its "figure of equilibrium". Finally, we have here the vera causa of 
that mysterious "lateral pressure" by which mountain ranges are known to be lifted up, but whose dynamical origin has till the present day remained shrouded in mystery.

Let us pass now to the contemplation of the nebula out of which our solar system is presumed to have been gestated and apply to its study the same principles we have just been considering; and for the sake of simplicity let us picture it as having sprung from a spontaneous explosion of an ancestral sun to a size, say, double the diameter of Neptune's orbit.

Naturally the first query to arise at this point is as to whether a disintegrated cloud of dust can ever fairly be treated as a parallel case to that of a compact globe like our earth. To this question I reply in the affirmative, basing my judgment on these considerations:

1. Enormous as is the breadth of the solar system, being no less than five thousand millions of miles across, its span is still only 1-5000 the distance of the nearest star. This extreme isolation Newton must have sensed (though, of course, he did not definitely know) and it doubtless misled him into the fatal error of construing the solar family as a universe unto itself wholly cut off from and independent of the rest of the cosmos. In my philosophy, on the contrary, this isolation is an essential factor toward constituting our system into a gravitational unit, that is to say, a group of associated bodies tethered together by their mutual attractions and seeking their lowest common center of gravity with reference to the goal of their cosmic fall.

2. In its quality of penetration, gravitation is unique. It does not vary with the area of the surface presented, but with the mass: a nail standing on its head on the scale weighs precisely the same as when lying on its side. For this reason a solid body like the earth reacts to the Prime Resultant (i. e., the stellar resultant belonging specifically to our system) as "molecularly" as though scattered into cosmic dust. Herein lies the dif- 
ference, however, that whereas the planet necessarily revolves as a solid piece, every individual particle of the nebula revolves in a free orbit round the common center of gravity of the joint mass.

To cite a historical incident by way of helpful illustration: Until the year 1857, the rings of Saturn were supposed to be solid, but in that year James Clerk Maxwell wrote an essay, "On the Stability of Motion of Saturn's Rings" (winning thereby the Adams prize at Cambridge), in which he argued the thesis that they could not be stable if solid or liquid, and hence must consist of discrete particles. This conclusion was later confirmed by Professor J. E. Keeler by means of the spectroscope.

3. In my criticism of the Laplacian conception, it will be remembered, I pointed out how he sought to explain the remarkable coincidences and harmonies characterizing our system by the wholesale postulate that the nebula rotated of itself as one piece. I recognize as did he the necessity of this concert of movement, but instead of postulating the uncertain and impossible selfexistence of motion, I seek the explanation in a dynamical factor sufficiently powerful and comprehensive, not only to account for the starting of the gyrations, but to ensure automatically their manifold mutual adjustments and their continuance. Incidentally let me impress upon the reader that in postulating inherent motion Laplace was obliged to assume primordiality for his nebula, thereby implying an actual Beginning to the cosmos and definitely committing himself to the teaching that all nebulæ which show signs of rotation are necessarily primal. I, on the contrary, regard all nebulæ as intermediate forms between other states of existence, and amenable to natural laws without special privileges or immunities.

Let us return again to the contemplation of the nebula, which we assumed as being produced by a solar explosion. It could, perhaps, be fairly pictured as initially spherical in outline, but more probably it would 
be irregular and nondescript. Whatever its original shape, however, we have in the Prime Resultant a lever whereby to bring it into harmony and order. From its imaginary initial state of rest, it would, at the behest of the Prime Resultant, begin to fall, thereafter accelera ting its motion with lapse of time, each discrete particle accommodating itself to the vortical current by the necessity imposed upon it of contributing to the general balance.

Obviously, such a process of concerted equilibration implies two things as necessary consequences: (1) that the gyrations occur in the same direction, and (2) that, though they need not so begin, they must tend more and more toward rotundity of orbit.

Again, what should the natural figure of equilibrium of the nebula be when the process reaches the mature stage? To open the way to the answer of this question let me follow it with another: Given a miscellaneous group of solid objects free to seek their lowest common center of gravity, how must they arrange themselves so as to ensure that center being at its lowest level possible? Can there be any doubt of the answer? On the same plane, of course.

Thus far we have considered the two extremes, to wit, first, our own globe, as a simple body dropping into the void, the while automatically poising itself on its center of gravity, and, second, the solar system in its entirety going through the same procedure, but reacting somewhat differently because of the multiplicity of its detached members. Between these extremes are the subordinate systems-the earth-moon, the Jovian, the Saturnian, etc.-which also demand our consideration. Each of these minor systems is, by itself considered, a gravitational unit seeking its proximate, local, individual center of gravity, and like the parent system constitutes a separate vortex. It is not the planets, as such, but rather the centers of gravity of their vortices that revolve around our luminary. Is it too much to assume, provisionally, that our solar system is in turn a member 
of another vortex next higher in scale, that of one greater still, and so on to the greatest of all, the Milky Way itself?

But every rule, they say, has its exceptions, and is proved by them. How can we explain the elongated orbits of the comets and their generally high inclinations; or how the retrograde motions of certain of the satellites? Comets, as I shall later show, are not indigenous to our system, but are fragments hurled in our direction by exploded, or "new", stars. They consequently enter our nicely balanced order haphazardly, at all sorts of angles, and it requires time to drill them into line. As for the erratic satellites, these are invariably the outermost of their respective planets and their peculiarity is clearly due to nothing else than a conflict of impinging vortices.

The last problems of celestial motions yet to be considered are, (1) the cause of the sun's journey through space, and (2) the star streams so-called. These must be considered together, for it is obvious that an accurate knowledge of the first is as prime a requisite to a correct interpretation of stellar movements in the large as a clear conception of the earth's gyrations is to a just interpretation of the planetary scheme.

No principle of mechanics is more certain than that every action has its equal and opposite reaction, and that the integral rotation of the solar system around its axis, under the special principle of the gyroscope, peremptorily demands the recoil of the center of gravity of the system. Pursuing this clew, I have been enabled, by the utilization of existing data hitherto misinterpreted, to demonstrate the sun's path to be that of an immense spiral with a diameter of $1,530,000$ million miles and a total coil length of nearly seven trillions! Making due allowance for this heretofore unknown motion of our observation-platform, the alleged "star streams" and the "preferential motion of stars" turn out to be nothing more exciting than optical illusions due to our own change of place. Furthermore, it develops that the pre- 
cession of the equinoxes is not owing to the causes Newton assigned to that phenomenon, but rather to the earth's comradeship of the sun in his spiral descent; while the misapplied causes pertain instead to the more recently discovered phenomenon of latitude variations that is still awaiting elucidation.

Again. We are prepared to explain the phenomenon pointed out by Kapteyn, namely, that the velocity of the rotation of nebulæ and systems increases with the evolutional stage. The older a nebula, the longer, obviously, has it been subjected to the influence of its stellar resultant, so that not only is each falling at a faster rate than ever before, but by virtue of the torsional strains I have described, its velocity of rotation is likewise increasing. In brief, the stellar resultant is the evolving power.

\section{The Laws of Gravitation and Equilibrium}

One of the most disastrous as well as one of the most inexcusable blunders ever perpetrated by men of science is their denial of the principle of balancing to cosmic bodies. The principle of gravitation implies equilibration, and vice versa: the two are virtually identical. When the body is in stable equilibrium, it means that the action of gravitation on its one side exactly equals and cancels that on its other side: when the body is not in equilibrium it means that the force of gravitation on one side is greater than it is on the other. Hence it is the height of absurdity to suppose it to be possible for one arm of a balance-scale to ever be heavier than the other without the fact being indicated by a downward movement of that side. Equilibration on the part of a free body is the best, and in some cases the sole, evidence of gravity. An excess of weight on one side is a motive power of which, in a universe seething with dynamical activities and problems, we must aim to make the most, rather than to try to conjure it out of existence.

As between my conception of our solar system being a gravity-engine whirled round and round by the force of 
its own fall, and the Laplacian conception of a nebula "existing of itself", "incandescent by assumption", "rotating of its own accord", and centrifugalizing and ordering its parts by no more substantial a power than the scientific imagination-to which should our judgment incline us? But we do not need to rely solely upon deductive reasoning as a support for our new conviction, for the best and surest of proof, namely, an exact concordance between theory, observation and mathematical demonstration, is forthcoming. The problem here presented itself to me in this guise: If it be true that the gyrations of the planets around the sun are indeed pure and simple evolutions of equilibrism, then should not the laws governing their velocities, distances and periodic times coincide with the known laws of the ordinary lever or balance-arm revolving in a horizontal plane? To this preliminary question the answer was promptly ascertained to be in the negative. But, came this new reflection, our hypothesis is not that of Newton, to wit, that the plane of the ecliptic is stationary, but that the planets and the sun are falling. Do, then, their velocities, distances and periodic times conform to the laws of balanced weights revolving horizontally and falling at the same time? Here the answer was found to be triumphantly in the affirmative.

The mathematics involved in this demonstration is of the simplest and easily within the capacity of the highschool graduate to understand and verify for himself. As "by-products" it yields: (1) a new demonstration of the law of gravitation, (2) a demonstration of the law of areas, (3) a direct formula for the ratio of times to distances, and (4) a direct formula for the planetary velocities. In addition to these important results, the first law of Kepler, namely that the planets revolve in closed ellipses, is shown to be fundamentally false, although his second and third laws, which heretofore have been recognized as purely empirical, are substantially corroborated.

Now, the one peculiarity that distinguishes the velocities of falling bodies from most others with which 
we are acquainted is, that they accelerate, and this they do according to a well-settled law, to wit, their advance varies with the square of the time elapsed. From our premises, then, it follows that not only are the planets gyrating around the sun for the reasons given, but that they are travelling faster and faster from year to year and century to century. But how can we hope to prove this fact, seeing that all the movements are simultaneously accelerating? There is only one way that offers any prospect of success. This is, to pick out those circulating bodies whose times of revolution are most rapid and whose orbits vary most widely from the circle (the ideal curve for evenness of balance) in the hope of discovering some slight excess of speed. Our choice is soon made, and falls upon the moon our satellite, and Mercury a planet. Nor, fortunately, are we doomed to disappointment. For have we not found Young telling us about the secular acceleration of the moon's mean motion advancing with the square of the time, and have we not also learned from Poor that Mercury's perihelion progression is explicable on the supposition that the sun's gravity decreases in a slightly faster ratio than the square of the distance (of which there is no proof), the converse being (as I now show) that the centrifugal force correspondingly increases?

Again. The earth's line of apsides, which joins her perihelion and aphelion, has been proved not to point steadily, but to revolve, exceedingly slowly, in the same direction as the planet. Newton, himself, showed mathematically that just such an effect as this would follow an augmentation of the centrifugal force relatively to the centripetal. Now, this is exactly what the Prime Resultant supplies, for in every succeeding year the planet descends a matter of a score or so of miles farther than in the year immediately preceding.

\section{The Tides}

It cannot fail of the reader's perception that in thus invoking the principle of equilibrium as applying to 
cosmic bodies frankly declared to be falling through the void, I put myself in the position of challenging Newton's tidal theory. But let it not be forgotten that in doingthis I am marching in good company; for do not Young, Kelvin and Darwin-all staunch Newtonians-proclaim that "the statical theory becomes utterly unsatisfactory in regard to what actually takes place", that "both theories must be abandoned as satisfactory explanations of the true conditions of affairs," that "the problem is one of insoluble mystery", and that "it would seem then as if the tidal action of the moon was actually to repel the water instead of attracting it"?

Paradoxical as it may sound to those wedded to the old idea, I contend that the sun and not the moon is the vera causa of the tides. There is positively but one single circumstance that connects the moon with our tides, a circumstance which, when rationally considered, far from constituting a proof of the moon's claims, is instead a flat contradiction. This single circumstance consists in the fact that at any given port high tide occurs fifty-one minutes (on the average) later each succeeding day and the moon crosses the meridian from day to day in accordance with this same interval. On comparing the state of the tide, however, with the position of the moon it is found that when the latter is overhead there is actually low tide, while when she is some thirty degrees below the western horizon of the port, that port has flood tide. "It would seem then", to quote Darwin's words again, "as if the tidal action of the moon was actually to repel the water instead of attracting it; and we are driven to ask whether this result can possibly be consistent with the theory of universal gravitation".

From time immemorial it has been a tradition that the moon is somehow the cause of the tides; for this coincidence in the matter of the interval did not escape the sages of prehistoric races. With the natural zeal of the pioneer, Newton sought to explain this connection with his new solvent of universal gravitation. At the outset he had but these two facts to go by, (1) the coincidence 
in the interval between the moon's passages, and that between high tides, and (2) the contradiction, that high water occurs, not under the moon, but some thirty degrees below her horizon. As he then conceived it, his problem lay in harmonizing these two adverse facts with the traditional hypothesis of the moon being the true tidal cause, and with his own new theory of gravitation. He was thus presented with a choice between making the interval-harmonize with the positional factor, or bending the latter into accord with the former. Unfortunately for himself and the good of the science, he chose the latter course, and undertook to prove how, by attracting the waters under her, the moon creates the reverse phenomenon of elevating them at those places farthest removed from her. In other words, he resolved to make black white by proving the phenomenon of repulsion to be the effect of attraction. Is it to be wondered at, then, that after generations of observation of the physical facts this tidal theory should have proved as "much wrong as possible"?

Having begun wrong, Newton stumbled onward from one tragic blunder to another; in which course he has hitherto been followed with blind servility by his heroworshipping followers. Let us briefly recite his various steps:

The first obstacle he encountered was the fact that the sun's attraction upon the earth is some 180 times stronger than the moon's; why, then, should not the sun rather than the moon be the cause of the tides? Dismissing this latter suggestion as wholly unworthy of consideration, he seized upon the notion that in raising up the waters under her the moon really has to draw in the surplus waters from the marginal areas of the earth's disc. Arguing from this he concluded that the tidal forces of the sun and moon operate obliquely, and consequently that they vary, not as the inverse squares, but as the inverse cubes of their distances. Computing thus, he made out the moon's power to exceed the sun's in the ratio of about four to one. 
On checking up with the phenomena, however, he found that although the excess waters ought, under this ingenious theory, to $b e$ under the moon, they are actually found afar off at the marginal areas whence he had hypothesized them to have been withdrawn! In other words, by drawing the waters theoretically to where she is, the moon objectively forces them to where she isn't: by pulling them obliquely away from the marginal areas, she obliquely heaps them up at those very areas!

But Newton in the pursuit of his object was determined not to be downed, and so he invented still another expedient. The tide, he now said, is "dragged" around the earth, and, like all things that are dragged, it is naturally to be found at a distance behind the tractive power. When we ask, "Dragged from where"? he answers, "Dragged from the place where it was originally raised up". When we ask, "Where was it raised up?", he answers, "Under the moon." When again we question, "Here is the moon now over our heads, and here we are at the port; show us the tide in process of rising, for to us it looks rather as though 'the action of the moon was actually to repel the water instead of raising it.' How does the moon thus manage to drag about a tide which she manifestly never originated and from where it never existed? Or are the tides, perhaps, like rectilinear motions, self-existent? Or does the moon find it easier to drag tides than to raise them; and to drag them to where she isn't than to raise them where she is?"

Another obstacle that lay in Newton's path, and which he resolved to get rid of, was the law of equilibrium. A little reflection will show that if this law applies to the earth in its relation to the moon, then the former should depress her center of gravity moonward, or, in other words, transfer her lighter mobile parts (her ocean waters) from in front of the moon to the rear, thus lowering the water-level under the moon, in accordance with the physical fact. Newton saw this plainly enough, and he would no doubt have been glad to accept 
this solution save for this conflicting circumstance, namely, that in that case his improvised law of cubes would have to be ruled out and the main premise (as to the moon being the chief cause) would fall by the board.

Now, how was the law of lowest center originally discovered? Objects falling naturally through the air fall with such velocity that no human eye is quick and keen enough to follow their gyrations upon themselves. In order to discover the secret of their maneuvers, therefore, some true-born scientist in the dim past conceived the idea of slowing-up the fall of divers objects without at the same time depriving them of the freedom of turning upon themselves as Nature might prompt. In other words, he brought the evidence within the purview of human perceptions. Now, what did Newton do, with his vaunted vacuum-tube experiment? In gross defiance of all scientific rules and precedents he systematically set to work, not only to restore the natural difficulties which his predecessor had been at pains to overcome, but actually to accentuate them by every artificial means his ingenuity could devise. By limiting the choice of materials to glass, he incidentally shortened the practicable length of the fall of the objects to little more than a yard, abbreviated the time for critical observation to a small fraction of a second, and precluded the use of micrometric instruments. Not satisfied with all this, he exhausted the tube of its air and thereby made doubly sure of baffling the vision. If this mode of getting rid of unpalatable truths is sound and logical, why not abolish the compound microscope, as a means of destroying those disease germs of whose existence we should otherwise be blissfully ignorant?

\section{The Author's Theory}

As an alternative theory of the tides I submit the following:

In the course of her annual journeys around the sun the earth is compelled to change her angular position 
with reference to him and, in obedience to the law of equilibration, to depress her center of gravity sunward. Now, were she a solid body throughout, she would in the course of a short time settle into a fixed attitude and constantly turn the same face toward him, just as the moon does now toward us. However, the earth is not such a body, but is generously endowed with liquid oceans (less than five times as heavy as her solid parts), which she is compelled to shift constantly from the sunlit side in her efforts to preserve her center of gravity at the lowest point. It is this struggle for equilibrium, while all the time being forced to advance in her orbit, which I assign as the major cause of the earth's diurnal rotation; the other being the torsional influence of the Prime Resultant.

It is not only necessary to Nature's scheme that the earth respect her center of gravity, but the earth-moon system must also respect $i t s$. It follows that in shifting her oceans from place to place over her surface the earth must automatically take into account the angular position of the moon with reference to the sun, as well as her own lunar distance at the moment, in order that both equilibrating objects may be jointly accomplished. Under these conditions we should naturally expect to find the tidally-displaced waters seeking, not a place under the moon, but rather those "corners" of the earth farthest removed from her; just as the phenomena proclaim. Herein, then, lies not only the explanation of why "the moon seems to repel the waters under her", but also why she and the tide follow each other in such rhythmical sequence.

\section{The Planet Mars}

Until very recent years Mars' surface was supposed to be an arid desert, but during his last two or three oppositions the evidence-whether due to more critical study or more intelligent interpretation-has led to the conviction that it is exceptionally moist. This condition being admitted, the reason for construing the 
network of lines characterizing his surface as "irrigating canals" completely disappears, together with all the resulting speculations about the "existence of a Martian race" and their "high state of development". A different interpretation of these interesting marks is therefore in order. Inasmuch as I appear to have been the first to insist on the presence of extensive oceans on this planet (having been led to this conclusion by my theory of tidal rotation) I have ventured to invent a theory of my own to show how, under Mars' natural conditions, such lines might, and ought, to come into existence as a plain matter of physical necessity.

To begin with, Mars receives less than half as much solar heat, area for area, as our earth; and as we must estimate this ratio, not from the ordinary zero, but from the absolute $\left(-461^{\circ} \mathrm{F}\right.$.), his average climatic tempera-? ture cannot but be at least $200^{\circ}$ below our boiling point of water. It is therefore necessary to conclude that Mars' seas, rivers and lakes are frozen solid to a great depth the year round, and to reason about his seasonal changes as taking place upon this ice surface.

Here we might hastily suppose that under the conditions of so frigid a climate there could be no such thing as water evaporation, but that water once frozen would stay frozen. We must bear in mind, however, that gravity on Mars is only .38 of what it is on our planet and that, even conceding him as much air in proportion as the earth possesses, his atmospheric pressure cannot on the whole be more than one-seventh of ours. Furthermore, it seems reasonable to suppose that the gravity of a planet, regardless of the weight of atmospheric pressure, may affect the volatility of liquids and that, Mars' gravity being little more than a third of that of the earth, water on his surface may vaporize readily, and possibly? boil, at our freezing point.

Another significant factor demanding consideration here is, that the water-covered area on Mars is very much greater in comparison with his land surface than in the case of the earth. To many readers this statement may 
savor strongly of paradox, inasmuch as, under the rules of geometry, the surfaces of spheres increase only as the squares of their diameters, whereas their volumes increase as the cubes. That is to say, given supplies of water proportional to their masses, the share of each square mile of Mars' surface would be but four-ninths as large as of the like area of the earth's. While all this is very true, it is no less true that the extent to which water will spread does not depend solely on its quantity, but quite as much on the character of the topography of the surface on which it flows or rests. Now, one of the most striking peculiarities of Mars is his extraordinary smoothness as compared with either the earth or the moon. It is by reasoning such as this that I have been led to conclude that Mars' natural surface is half marsh and half open sea.

I take it that the interest displayed by the public in Mars centers entirely round the question as to whether it is inhabited by an intelligent race of beings; refute this hypothesis definitively and that interest will speedily die out. Whoever will give himself the trouble of weighing the evidence pro and con cannot but arrive at last at the conclusion that the Lowellian hypothesis rests solely on these two facts (if, indeed, they be facts), namely; (1) the presence on Mars of a seemingly systematic network of straight lines of very great length, with dots marking their numerous intersections, and (2) a total absence of seas and oceans. The theory holds that such geometrical accuracy as these lines exhibit betray a planning intelligence, that there must have been a pressing motive behind so elaborate an enterprise, and that that motive can have been nothing else than to utilize the meagre supply of water yet remaining. Ergo, the lines are cultivated strips of land bordering on canals.

Now, it is a sound legal maxim that when the reason for the law ceases the law itself ceases. Since closer observation, then, reveals indisputable evidences of ample water supplies on our neighboring planet, the motive for the building of the alleged canals fails, and the 
whole elaborate theory erected by Lowell falls away with it. Such evidence will be adduced in plenty in a later chapter.

But if these lines undeniably exist, and if they are not designed for irrigation, what in truth are they? Shall we still cling to the notion that they cannot exist naturally, and cast about for some other motive to explain them?

$\mathrm{My}$ conception is that these lines veritably exist, together with the dots at their intersections, but, in view of the immense difficulties in the work of observation, I do not accept these marks as anything like as regular and uniform as the Lowellians would have us believe. Assuming them to be due to natural causes, the question at once confronts us as to whether such geometrical outlines as we here have to deal with cannot more easily come into existence on an ocean surface than on a land surface. Heretofore it has been precipitately assumed that marks of any sort can be retained permanently only on the continental areas, and this has been the chief reason (inasmuch as the lines principally affect what appear to be ocean surfaces) why Mars has so long been believed to be a desert planet.

Having carefully weighed all the foregoing matters separately, as well in their mutual relations, I have arrived at the following conclusions:

1. That Mars is endowed as generously as the earth with atmosphere and oceans in proportion to his mass.

2. That on account of his greater distance from the $?$ sun and his consequent lack of warmth, his oceans are frozen to at least one-half their depth.

3 . That the remainder of his surface (corresponding with our continental areas) because of its slight elevation above the ocean level, is of a marshy nature, much diversified with ponds, lakes, inlets and connecting straits.

4. That with the alternation of seasons, and of day and night, rain and snow fall upon this ice crust and give 
rise to a great litter of loose detritus, which, by the washing back and forth of the tides, automatically arranges itself in long and fairly regular ribs, which not only traverse the ocean expanses but even, in a modified way, invade the marshy areas as far inland as the arms of the sea penetrate.

\section{The Moon}

Whereas the surface of Mars is remarkable for its smoothness, that of the moon is astoundingly rugged. Why should this be so? It would be natural to suppose that the difference in size might be the cause, but the moment we include a third body, our earth, for the purpose of comparison, we find this inference to be a mistake; for though much larger than either of the other two, our planet is neither the first in roughness nor in smoothness. Which of them constitutes the exception, and why? My answer is, the moon.

In casting about for points of difference and resemblance between these three bodies we cannot but be struck by this one outstanding fact, namely, that whereas the earth and Mars rotate in front of the sun in the space of about 24 hours, the moon so rotates but once in 28 days. This peculiarity gives every point on her surface a day one of our fortnights in length, followed by a night of the same duration. In fact, her day is really a summer and her night a winter. Now, there is no necessary equivalence between her summer and her winter, and one season is just as likely to overbalance the other as vice versa. This question only the resulting phenomena can decide.

Let us assume, then, the severity of the lunar winter to overmatch the geniality of her summer, and reason out what should be the natural effects; presupposing, first, that her natural surface was originally as smooth as that of Mars and, second, that she was once blessed with her proportionate share of air and water. Imagine, now, if you please, that during the first winter there fell on every foot of her land surface a depth of five feet of 
snow and that during the succeeding summer the whole of it was melted away save for a remnant, say, of one inch; that during the second winter another five feet fell, and again was cleared away save for another, additional inch, and so on indefinitely. Obviously, the logical outcome of such a process could not be otherwise than eventually to transform the entire lunar supply of water into snow and ice; and not only that, but to transpose it from its natural pools and beds onto the land.

This "disappearance" of the moon's oceans involves, of course, the simultaneous swallowing up of her a.tmosphere. Meteorologists inform us that a single inch of rainfall is equivalent to a 10 -inch snowfall, which is tantamount to saying that in changing from water into snowflakes a cubic foot of the former absorbs about 10 cubic feet of air.

Given the unlimited supply of building material thus provided, it becomes a simple and fascinating pastime to reconstruct in imagination the characteristic lunar sculpturings that heretofore have baffled selenographers: plains become mesas, deep ponds graduate into cavernous craters, islands grow into mountains or mountain ranges, rivers erect their level banks into precipitous gorges, great lakes disguise themselves as walled-plains, and so on ad infinitum. As for the changes of shading of certain areas from white to drab, and back again, they are obviously due to the sopping of the low areas with waters of thaw, followed by freezing and, later still, by belated falls of fresh snow. Again, inasmuch as there can be no building up of snow-mounds on the beds of the maria until they become uncovered, we find in these places the dwarfed and "ruined" replica of all the types that characterize their giant brethren on what originally was the land surface.

\section{THE PRINCIPLE OF DISPERSION OR EXPLOSION}

It has long been the dream of mankind to discover some method of utilizing gravity to do work contin- 
uously, but so far without success. The difficulty has always been found to lie in the impossibility of providing a clear track for the descending weight; the earth herself persistently barring the way. We have seen, however, how Nature, wiser than man, utilizes the divergent rays of the far distant stellar attractions to operate our cosmic machine and keep it going through the ages.

But here arises a misgiving: Must not this process of mutual approach of the stars, however ingeniously prolonged, end in any case in their final collision and common burial? Will not Nature encounter, sooner or later, precisely the same difficulty as man and find her operating weights blocked by the sources of gravitation themselves getting in the way? My answer to this is, No.

In our discussion of the causes of celestial motions we saw with what improvidence Newtonians cast aside the cumulative attraction of the stars as a thing of no account, or, worse still, as an actual stumbling-block to the smooth working out of their adynamic theories. Here again, in another field, we come upon a second example of how blunderingly they mistake gold for dross. Let me explain myself :

According to the letter of the law, gravitation increases inversely as the square of the distance. If this means anything, it means that when a cosmic body, say the sun, reaches its most condensed and compact state, its integral gravitation attains its maximum. Our scientists inform us that in progressing toward this compacted condition gravity causes the collapse of the nebula and by so doing converts its "potential" energy into "kinetic" energy, or heat. Now, common sense should lead us to conclude that when the power (in this case gravitation) is capable of producing certain well-defined thermal effects, then when said power is increased to its maximum it ought to produce maximum effects; nor this for a single moment only, but as long as the power remains at par. This, at least, is my contention, though it is quite the opposite of what orthodox science is now teaching. Its teaching is that force and energy are two 
different things; the former not being "conserved" while the latter is. Hence, no matter how "strong", gravity may be, they say, it cannot find resurrection in the form of heat or any other sort of power, but dies with the act it performs, whereas "energy", they add, can never die, but lives forever in a continuous chain of transmutations. Should you think to answer by saying: "But when an object falls and strikes the ground, does it not produce heat? And is its fall not due to gravity? And is not gravity, therefore, in this instance at least, conserved? they will answer you, as does Mr. Soddy, (v. p. 16) by denying that gravity is responsible for the fall and declaring it to be a mere "fiction." Against such resources in argument I cannot hope to prevail. Gravitation is a force, or it is not force. If only a fiction, let us blot it out of scientific parlance and change the law to read: A fiction varies inversely as the square of the distance and directly as the product of the masses. I reiterate, that just as our scientists wantonly thrust aside the greatest of dynamical factors, the stellar resultant, in their interpretation of the celestial motions, so do they here repudiate the incomparably great heat-generating factor of static self-compression. The heat thus generated-in perpetuity-I call gravistatic heat.

Compressive forces, however, have other dynamical possibilities. You may detonate a stick of dynamite by warming it, by striking it, or by immersing it in certain acids. You may also explode it by gradually increased pressure. Reasoning from multitudinous analogies, is there not every ground for assuming that all substances are explosible-given only the necessary degree of pressure? This, at least, is my hypothesis, and I base it on the well-known converse scientific principle that every gas has what is called its critical temperature point at which no amount of applied pressure is able to reduce it to a solid or liquid state. Now, taking the sun's intense temperature merely. as an overt fact without bothering about its explanation, his central temperatures 
are demonstrably so high as to guarantee the presence there of an immense accumulation of superheated gases defying condensation by the superincumbent pressures, great though these undoubtedly are. To complete my conception, then, we have only to suppose that when a given star attains excessive size, it generates so great a quantity of gas within itself that the equilibrium between the forces of compression and expansion is destroyed and the star disrupts into a "nova", with its necessary accompaniment of a nebula. It is not necessary, nor shall I attempt, to define dogmatically the severity of such explosions, leaving the phenomena to speak for themselves. This much, however, I take as certain, that their dispersive effects commensurate with Nature's needs for self-preservation against the constricting menace of concentration.

\section{The Sun}

My conception of the sun is that of an immense central chamber of superheated gases supporting, and in turn imprisoned by, a huge shell of molten matter. In its general structure it may be likened to a cocoanut, or better still to a cantaloupe, except that the solar shell is relatively much thinner, being in fact only about one twentieth of the radius.

That the sun is very eruptive, not merely superficially, but profoundly, is sufficiently attested by the phenomena called "prominences", some of which have becn observed to rise with initial velocities of as much as 100 miles a second and to reach heights upward of 200,000 miles. From this we may infer two things, (1) the deep-seatedness of the explosions that give these prominences birth, and (2) that in the course of time the entire substance of the sun has been carried out above his surface, perhaps repeatedly, and thence rained down upon his photosphere. Let us therefore trace the course of the various materials from the surface of the photosphere as the starting point. 
Here we are confronted with three controlling conditions, namely: (1) Plasticity of the solar shell, (2) Gradually increasing temperatures and pressures (explosive) as we descend below the surface, and (3) Great diversity between our materials in the two decisive respects of volatility and specific gravity (atomic weight). We thus have the elements of a sort of obstacle race, in which the test of merit consists in ascertaining which of the entrant materials can penetrate farthest before undergoing re-volatilization and re-expulsion. Obviously, that particular element or compound that possesses at once the highest specific gravity and the strongest refractoriness (qualities usually associated) may sink clear through the shell into the central retort, while the rest will penetrate in like manner to their several appropriate levels. In fine, the shell progresses toward homogeneity and refractoriness as we descend, and herein we behold in our sun, not alone a fountain of light and heat, but a natural smelter by which Nature assorts-for man's sake?-her diversified materials. These deductions suggest to us the chemical secrets of meteorites and the cause of the natural segregation of our ore deposits.

Going back to our obstacle race, we note that, inasmuch as each substance has its separate characteristics, its eruptive interval is lengthened or shortened accordingly. The cycle of the most volatile will be so short as to be in effect continuous and the resulting eruptions or geysers so countless as rather to deserve the title of ebullitions. Such are the so-called "rice grains". On the ther hand, the longer it takes a given substance, or class of substances, to round their cycle, the longer will be the interval between displays, the deeper the seat of the blast, and the more distinct the period and resulting phenomena. Of this nature are the "sun-spots", whose average period is slightly more than eleven years. There are, however, many shorter cycles, though not as yet clearly defined, and one, or perhaps more, long, epoch-creating ones, of which we have no historical but 
only geological proofs. I refer here to those cataclysmic explosions which, by their sudden cooling of our solar furnace, ushered in the climatic crises known as ice ages.

The foregoing interpretation of our luminary (a typical star) makes clear that, as such, he is as eternal as matter itself. Granting the indestructibility of matter, the indestructibility of the nature of matter, the non-impairment of gravitation, the constancy of the sun's mass, the absoluteness of the coldness of cosmic space-things which no one will care to debate-the process outlined is one of perpetuity. That I mention the absoluteness of spatial cold as a condition toward the generation of solar heat may seem to some strange; but a little reflection will reveal it to be the negative pole to Nature's thermal battery; for without its aid the expelled gases would dissipate themselves in space instead of suffering re-condensation and being regularly thrown back upon the sun or star.

The tremendous activity of the photosphere and the enormous proportions of the corona demonstrate the extreme density the latter must possess. This is a sore point to the Newtonian theorist, for this reason: In order to render his theory of planetary motions at all plausible, Newton was obliged to assume the ether to be absolutely non-resistant, else there was no disguising. the fact that the planets would inevitably fall into the sun in short order. Now, of all heavenly bodies the comets are recognized as the most ethereal, yet observation has proved that some of them that passed through the densest part of this corona, and within 40,000 miles of the solar surface, not only ran this gauntlet safely, but even showed no slackening of speed! Compelled thus to choose between theory and ocular evidence, astronomers decide in favor of the former, and make believe the corona to consist of nothing but the thinnest of gases and to be as non-resistant as the ether itself. Under my theory of the Prime Resultant, on the other hand, the body of the sun, the corona and the comets are all component parts of the systemal vortex and rotate 
compulsorily as a matter of equilibration, so that we are under no temptation to gloze the plain facts.

It is reasonable to suppose the sun and the earth to be composed, not only of the same kinds of materials, but also in about the same proportions. Then why, remembering the vastly greater self-compressive power of the sun, is his density only one-fourth that of our planet? Heretofore the reason given has been, that the sun is "contracting" in size and that he is in process of transmuting his original "energy of position" into "kinetic energy". No one, strange to say, has ever suggested the plain, common-sense view that the sun is distended because he is hot; for what physical fact is better known than that heat expands? In my conception the sun is a mechanical furnace in which his power of selfcompression by gravity is constantly churning his substances over and over again from fluid into gas, and, with the help of the cold of space, from gas back again into fluid. The more massive he becomes, then, the hotter he grows. Moreover, the hotter he grows, the more abundant the internal gases he generates within himself; the more he becomes distended; the greater the number of his elements kept in a perpetually gaseous state; the more refractory the average constitution of his shell, and the thinner, more fluid and well-mixed it is; the freer his surface ebullitions, and finally, the nearer he approaches the climax of bursting into the nebular state.

Again. To assert that the sun is, by growth, becoming hotter, is equivalent to saying that he is also increasing in brightness. But in the case of the sun (or any star, of course) here is this special reason: The more refractory to volatilization a substance, the more intensely bright is it when raised to incandescence; and since, by premise, the shell gradually becomes more and more refractory in constitution, while at the same time the ebullitions in it become more rapid, mere brightness accentuates into brilliance.

Let it not be supposed, however, that our sun can explode but once, or that he cannot undergo explosion until 
he attains the theoretical maximum I have indicated. What I mean to say is that even in the case of the most favorable and regular evolution of a star that may be imagined, its ultimate passage by disruption into a riebula is inevitable. Nor does this mean that the star, as such, necessarily vanishes. On the contrary, our sun, for example, has shattered himself thousands of times by virtue of deep-seated explosions, but with the exception of a very small fraction of his mass he has as often pulled himself together again, within perhaps a year's time, leaving the missing fraction in the shape of a nebulous cloud to be swept up by his planets as they leisurely continue their rounds under the steadying guidance of the Prime Resultant. Thus does Nature utilize gravity as her detonating agent for the dispersal of her overgrown stars and to keep the path of descent clear of obstructions.

If it were possible to step out of our system and view it from a great distance, which of its myriad bodies would appear to travel most slowly? Undoubtedly, the sun. For the sun has only the one translational movement, while his planets not only possess that in common with him, but, besides, revolve rapidly around him at the same time. Which, again, travels the faster, the earth or the moon? The latter, because in addition to partaking of the former's movement around the sun it has, superadded, its rotation around our planet. In general, then, it can be stated that the larger the star the slower it travels. Moreover, it follows mathematically from the law of gravitation that where two bodies approach each other, under their mutual attraction, their velocities vary inversely as the square roots of their respective masses.

Some of my readers, remembering that Jupiter and Saturn are recorded as being less dense than the sun, may suppose me to be in error in asserting that the density in bodies of stellar size is inversely a function of the mass. These particular discrepancies, however, are easily reconciled when we take into consideration the 
circumstance that the two planets are visible to us, not by their own light, but by that of the sun reflected from their very heavy atmospheres, which latter we thus necessarily include in appraising their volumes. Were the sun's disc to be suddenly darkened, and were we then to behold him by the light of another star, we should not be able to see his photospheric disc at all, but in its place there would come a dull disc of greatly enlarged dimensions, due to the inclusion of his dense corona. Under these parallel conditions his density as compared with Jupiter and Saturn would, of course, be very much reduced.

The rotation of the sun on his axis follows clearly enough from the principle of the Prime Resultant and its torsional stresses, but his so-called equatorial acceleration is a phenomenon that requires additional explanation. Under the strict letter of Kepler's third law, a planet near the sun's surface would revolve more than two hundred times as fast as does a spot on his photosphere. The reason for this apparent contradiction of my theory lies in the fact that the sun is astride the fulcrum of the system and consequently trims the general balance, not merely by rotating, but also by oscillating and tilting back and forth across the medial line. Naturally, the heart of the sun partakes most of the latter maneuver, while his superficial equatorial rings, from the photosphere on upward through the corona, partake most of the rotating tendency; and of these rings the uppermost, i. e., the farthest from his axis, obvicusly travels the fastest of all.

\section{The Stars}

It goes without saying that the only reliable method of interpreting stellar phenomena is to possess ourselves first of a clear knowledge as to what a star is in essence. Fortunately for our purpose there is one star very near to us-our sun-which we can view and study at close range and whose life-history is plainly written upon the stony tablets of our Mother Earth. 
In a certain sense every meteor that roams interstellar space can be called a star, because it may eventually develop into one; just as a babe may become a man, or a larva a moth. In my vocabulary, however, $a$ star is a heavenly body already so large as by its gravistatic heat to be self-luminous. In the animal and vegetable worlds the more highly developed types are the more complex, but, strange to say, this rule is reversed among the stars, for the largest and best developed are the very simplest in structure. Of this sort are the so-called Spectral-class B stars, which let us now consider in some detail by way of illustrating my method of star interpretation.

Astrophysicists in classifying stars by their spectra began to do so at a period when the Nebular Hypothesis of Laplace was universally accepted, and although that conception is now generally discredited, the old classification still prevails. It was assumed that stars freshly born of their nebulæ are then at their best and brightest, and from that time onward gradually cool, fade and decay. In this way the mass of stars as a possible key to their several peculiarities was altogether lost sight of and the spurious standard of age substituted. Thus it came about that the spectra of the very brightest stars, intrinsically, are denominated "early", while those of the duller sort are called "late". This to me seems a very unfortunate error, inasmuch as it not only decides the issues in advance of the investigation, but raises up one more prejudice to add to the multitude already crowding the path of progress. The age of a star has nothing to do with a star's brightness or dullness, save to the extent that the longer it has been in existence the more chance has it had to grow by feeding upon the flotsam of space. My rule is, simply, that the attributes of stars are functioned upon their mass. The bigger the star, caeteris paribus, the slower its velocity, the rarer its density, the hotter its temperature, the "earlier" its spectrum, the brighter and steadier its light, and the longer its distinguishing cycle of variation. 
Granting my contentions that gravitational self-compression perpetually generates heat, and that all substances are susceptible to explosion under adequate pressures, it follows:

1. That Class B stars (assuming these to be the most massive) are the hottest of all.

2. This being the case, the central gaseous chamber must be greatly enlarged and the shell correspondingly thinned, (1) by the enhancement of the temperature and consequent.intensified buoyancy of the imprisoned gases, (2) by the distillation of a maximum number of elements and compounds out of the shell, and (3) by the extension of the superficial area over which the magma of the residual shell has to be distributed.

3. Not only, however, is the shell relatively thinned in the sense of linear measurement, it is also rendered thinner in the matter of consistency, for the vastly augmented temperature fuses all of its component substances until they flow like molten steel from a blast furnace.

4. Now, it is common knowledge that the more refractory to volatilization a mineral substance is, the hotter and brighter it is when raised to incandescence; and there are degrees even to this state of incandescence, comparatively small increases of temperature returning truly amazing rewards in the way of enhanced illumination.

5. As our giant B star grows larger and larger, its shell becomes thinner in consistency, and thinner and thinner dimensionally (at least relatively to its diameter), thereby rendering it thermally unstable and its tenure of life insecure. Eventually the equilibrium between the internal buoyancy on the one hand and the weight and cohesion of the shell on the other is overcome by the former, the shell suffers rupture, and its substance is spattered to great distances, giving rise to a nebula of great size. Nebulæ thus produced are apt to be 
regular in outline. Such, however, are not the largest specimens of their genus; for there are a few nebulæ, e.g., Orion, that have probably been produced by the contemporaneous explosion (or perhaps collision) of more than one star.

6. It is a grave mistake to estimate the mass of a star by the amount of light it emits, as foolish, indeed, as it would be to select artificial lights by their physical size instead of by their intensity. Astronomers in general labor under the delusion that there is no natural limit to the sizes of stars, hence we find them speaking of such a star as Canopus, for example, as being a "thousand times the mass of our sun." Could we behold the surface of a B star at close range, we should see it, not as a smooth expanse, but as one stippled with millions upon millions of jets of incandescent materials rising thousands of miles skyward, literally pumping out the star's gravistatic heat. It may well be doubted whether a star can exist, as such, with a mass more than a hundred times that of our sun.

7. Another feature respecting these B stars is the broadening or blurring of their spectral lines, indicating at the same moment approach and recession, or, as the astrophysicist would express it, both minus and plus radial motions. This phenomenon is obviously due to the combination of jets, some in the act of rising and others in the act falling; both impressions, of course, registering themselves on the photographic film together.

8. Inasmuch as, like all other things, stars can grow only where there is food for growth, we should expect to find a greater proportionate number of these giant B stars in the Milky Way than in the regions of scantier pasturage. This is just what observation proves to be the case. Incidentally it may be stated as a general rule, that the "older" their spectra, the larger the membership of a given class, for according to my theory the so-called "old" stars are those of small mass. 
9. In the maturest stars the shell is so well diffused and fluid throughout that ebullition within it can go on freely, and consequently they experience no intermediate extraordinary eruptions, which are the only sort, of course, that can explain variability. In the second grade of stars, again, the shell is also very fluid, but not clear through, as in the first grade, so that there is a tendency to clog in the lowest strata, but only at very great intervals, while ebullition in the superincumbent layers proceeds freely. At the other end of the scale, on the other hand, we find the shell possessing a comparatively shallow fluid covering in which the distinctive variation-producing eruptions accordingly take place at only a slight depth, and, consequently, with greater frequency. As general conclusions, then, we may state, (1) that the variability of stars is functioned on their mass, (2) that the maturest stars have no distinct variation save that of final disruption into nebulæ, and (3) that the smaller the star the shorter and more uniform its period of display.

\section{The Genesis of the Solar System}

Nature, like man, often has a variety of ways for accomplishing a given result. Thus she may level the tree with the sword of her lightning, the breath of her storm or by decay, or the woodman may fell it with the blows of his axe or the blast of dynamite. That the Great Pyramids were built by human hands we well know, yet we count it no disgrace that our historians and engineers alike have failed to discover their architectural secret. So in our efforts to fathom the precise genesis of the solar system many details will doubtless have to remain unresolved, even though we may be able to define the methods which lay open to Nature's choice.

1. We may imagine, to begin with, a great nondescript cloud of primordial cosmic dust suddenly being galvanized into action by the divine edict, Fiat Gravitas, and then crystallizing into nuclei here and there; some, 
like the planets, afterward waxing into bodies of great magnitude; others, like the satellites, into orbs of lesser size, and yet another giant world-the sun. But concurrently with this process of agglomeration, from the very beginning, we must conceive of our cloud (picture it as large at the outset as you please) as in the act of falling and gradually accelerating in velocity, incidentally resolving itself into a main vortex, and many subordinate vortices, under the torsional and suctional influence of the Prime Resultant.

2. Or, secondly, we may view the sun as first developing to his full stature and later exploding at intervals; each time throwing out a small fraction of his mass to form a nebula. During the earlier of these crises we may postulate the nuclei of all the planets and satellites, or of as many of them as we choose, to have come into being and, under the constraining hand of the Prime Resultant, to have finally resolved themselves into an equilibrated general unit and subordinate units. This order once firmly established, we may picture a long series of violent disruptions of the luminary, each resulting in a tenuous nebulous cloud filling the Neptunian orbit,and then conceive of these nebulæ as being successively swept up by the gravitational suctions of the circling planets - a chain of events not yet ended.

3. We may differentiate between the superior and inferior planets, drawing the genesis of the latter from the sun, under the second described method, and postulating the former as independently evolved at a considerable distance away, and subsequently sucked bodily into the solar maelstrom.

There is no fundamental inconsistency between these methods, and there is good reason to believe that each of them has borne a share in the general result. Thus, the first may suggest the initial step, the second may account for the asteroids, and for the ore-pockets found on the earth's surface, and the third may serve to elucidate Bode's law and supply the answer to the more general 
problems affecting the diversity of the planets in the particulars of size and density and the number of their circulating attendants.

From Nebula to Nebula-from star-dust to stardust-through comet, meteor, satellite, planet, sun, then back into nebula again: this is the life-span of our, of every, planetary system: the ultimate physical cycle within the comprehension of finite man: the last round short of Infinity itself!

\section{The Destiny of the Universe}

Reverently acknowledging that the Hand that created the universe can at will destroy and annihilate it, I hold that, viewed as a purely physical contrivance, it is potentially indestructible, imperishable and eternal; that all the activities of the universe, including the planetary motions both of orbital and axial rotation, the fiight of the sun, the proper motions of stars, the gyrations of the nebulæ, the heat and light of the sun and stars, are born of the moment, and from moment to moment, and are not the inheritance from a dead and mythical past; that were the earth conceived to be gently brought for an instant to complete rest, it would on being released not drop to the sun, but would gradually recover its lost momentum; that were the sun by fiat chilled to zero, he would in the next-the fiat being lifted-immediately begin the generation of heat within himself until in a calculable period he would be radiating with the same intensity as now ; that, in brief, were the whole universe to doze off into cold and comatose inaction, it would on waking, by virtue of its inherent vitality, resume its suspended operations with only a temporary dislocation of its parts and a rapid return to its present swing and vigor. 


\section{III}

\section{NEWTON'S THEORY OF PLANETARY MOTIONS}

$\mathrm{P}$

ERHAPS the first system of celestial motions meriting the name scientific was that devised by Claudius Ptolemy, who lived about the middle of the second century of our era. According to him, the earth was the center of the universe around which revolved daily, in the order of their supposed distances from it, the Moon, Mereury, Venus, the Sun, Mars, Jupiter, Saturn, and the stars. Beyond the stars, again, was the Primum Mobile, or Prime Mover. To prevent the planets and stars from falling down upon the earth, Ptolemy conceived of them as set in separate crystalline spheres; and these latter, he supposed, by the noises of their rotation, produced the "music of the' spheres". Strange to say, this notion of celestial harmonies survived the discovery of Copernicus, for Kepler speaks of it, and assigns to Jupiter and Saturn the bass, to Mars the tenor, to Venus the contralto, and to Mercury the soprano parts in this heavenly chorus.

Having assumed that all the celestial bodies rotated around our earth, it was quite natural to surmise, in the first instance, that they did so in exact circles. In our practical age we should be apt to do the same, were we approaching the problem for the first time; but if we should then speedily find that observation materially contradicted theory, we would doubtless promptly try out some other sort of curve in the effort to find a short cut. 
This simple expedient the ancients seem never to have thought of, for those were the halcyon days of deductive philosophy, when man sought to solve nature by presumptuously trying to read the mind of the Infinite by divination, instead of indirectly, through His works. They reasoned, metaphysically, that the Creator would not choose any but the "perfect curve" for the paths of the celestial bodies; and they would have accounted it blasphemy in anyone who might have suggested an alternative idea. Accordingly, when systematic observation showed certain vagaries of movement on the part of the planets, instead of adopting the obvious course and experimenting with other possible curves, they invented the device known as epicycles; - that is to say, they imagined the planet, in addition to revolving in one great circle, to revolve also in a second, smaller circle, whose center, rather than the planet's center, progressed along the circumference of the main curve. One such epicycle proving insufficient, nowise daunted, they postulated a second epicycle grafted upon the first, and so on indefinitely, until, by the time of Copernicus, they had as many as seventy such arrangements piled one upon the other!

Such, then, was the state of theoretical astronomy at the close of the regime of the Ptolemaic system, whose rule, though not actually ended, was at least foredoomed by the publication of Nicholas Copernicus' (1473-1543) great work, De Orbium Coelestium Revolutionibus, in the very year of the author's death. In this treatise Copernicus taught that the sun, and not the earth, is the center of our system, that the moon revolves around the earth, and that the earth and all the rest of the then known planets revolve around the sun in circular orbits. The only material mistake he made lay in perpetuating this last doctrine, inherited, as it was, from the older system; but in extenuation it should be mentioned that the general reform brought about by him was so basic as to render for a time unavailable the tangled skein of mathematical material accumulated by his predecessors.

As things turned out, it would have been a lasting reflection on the perspicacity of philosophers had the dis- 
coveries of Copernicus been delayed a half century or so longer; for by that time Galileo (1564-1642) had improved the telescope, and with its help had observed the phases of Venus and followed the satellite system of Jupiter through several cycles of rotation. Had Copernicus been in possession of these significant facts, much of the glory of his achievement would have been lost.

Fortunately the cycle-and-epicycle fancy was likewise destined not to survive long; for John Kepler (15711630 ), in the year 1627, gave it its quietus when he published his three laws of planetary motion, as follows:

1. The planets revolve in ellipses.

2. The radius vector passes over equal areas in equal times.

3. The cubes of their mean distances are proportional to the squares of their periodic times.

Although later researches have shown these laws not to be exactly true, they are so close an approximation to the truth that modern astronomers seem to have tacitly agreed among themselves to shut their eyes to the observed discrepancies as immaterial-alas, to the very great detriment of the science! 'In estimating the true value of Kepler's contribution to astronomy, it is important to remember that his discoveries were purely empirical, and that they were arrived at without reference either to the principle of gravitation or to the fact of the sun's flight. The story of Kepler's labors and privations in the pursuit of his quest is a tragic one, all the more so because of the perverse genius he displayed in so long eluding the obvious. If only he had had the sagacity when he began his labors to ask himself the seemingly self-propounding question,-May not the planets move in curves other than circles? - he would unquestionably have shortened to a period of weeks, or even days, the score of years that his task actually consumed. What a travesty on human intelligence! Fourteen centuries to evolve the simple idea that the earth, instead of the universe, rotates on its axis once in twenty-four hours! Fifteen centuries to prompt 
the query,-Why may not the planetary orbits be other than circular? These two ideas, epochal though they proved, were by no means strokes of genius, but only the prosaic promptings of common sense erupting through the smothering strata of superstition, tradition, and mathematical abstrusities that the wiseacres of earlier centuries had heaped up. So far as the mathematical proof was concerned, that was only a matter of rules and industry, once given the clue. Clearly, the two most important lessons of astronomical history are, (1) that vigilant skepticism is the price of progress, and (2) that investigation should begin with the near and obvious.

The next great name in the development of the science is that of René Descartes (1596-1650), although he is seldom thought of as an astronomer, but rather as a philosopher and mathematician. I mention him here, partly because it was his cult whose ascendancy over the world of thought for almost a century after his death was great enough to bar out of England's schools the teachings of her own son, Sir Isaac Newton (1642-1727), during the whole of that philosopher's long life, and partly because he, Descartes, was the originator of the Vortical theory of planetary motions; a theory which, though utterly impractical as he conceived it, we shall nevertheless find to be true in principle when combined with the workings of universal gravitation.

It is with sincere regret that I must confess my inability to share the world's extravagant estimate of Newton as a philosophical astronomer. As a mathematician he may possibly have been supreme; but of this I am not competent to judge. Was it not Huxley who first said that you cannot take out of mathematics more than you put in it-meaning, that if you start figuring upon false premises you cannot arrive at useful results? Because a man is a mathematical genius, does not signify that he is equally great, or even great at all, as a theorist or constructor. Ptolemy was a mathematician of the first order, but his crystalline spheres and epicycles were, for all that, fantastic unrealities. In our day the Nebular Hypothesis of Laplace (perhaps a greater natural mathe- 
matician than Newton) was universally hailed as the supremest conception of man, but we have lived to see it laid aside. Newton, too, $\mathrm{O}$ hero-worshiping reader, was ordinary flesh and blood like ourselves, and fallible even as Ptolemy and Laplace. Not only that, but he lived in an age when the bible was still regarded as a scientific authority; when the Inquisition was not yet dead; when witch-burning was a religious rite; when the earth and the entire solar system were generally believed to be only a few thousand years old; when Uranus, Neptune and the asteroids had not yet been discovered; when the sun's motion was not even guessed; when the spectroscope was undreamed of; before the mechanical theory of heat was discovered; before the accuracy of his law of the inverse square had been impugned; and before his theory of tides had been weighed in the balance and found wanting. Shall we now wave aside these important considerations, and, with the bigot's finality, proclaim that "Newton's science is good enough for us", and that, hedged about though he was with the narrow limitations of his age, he could commit no error? Now that we know so many more basic astronomical facts than Newton did, why should we seek to crowd them all into the superstructure of theory only, rather than to employ them in broadening, strengthening, repairing, and remodeling the foundations that stand in such sore need of attention?

It seems wonderfully strange to me that, of all the brilliant minds that have heretofore applied themselves to the theory of astronomy since Newton, not one has so much as suggested the utilization of the energy inherent in our cosmic fall to account for the gyrations of the planets and satellites, and especially for their multitudinous concordances. Following the lead of Newton, one after another of them has fallen blindly into line and kept in the same old rut. Newton, of course, is more to be excused than the rest; for he was not in possession of certain essential factors, and, besides, he was so far ahead of his own generation that not even his modest postulate that gravitation extends as far as the moon was accepted by it during his lifetime, though he survived, as was 
stated before, the publication of his Principia for as much as four decades. The oversight, futhermore, appears all the greater when we remember with what assiduity mathematicians have devoted themselves to the investigation of the purely academical and altogether speculative "fourth dimension of space", the while remaining stone blind to this genuine third dynamical dimension of the real cosmos. To them, just as to Newton, the solar system is, to all intents and purposes, a world of two dimensions, in which the mutual attractions of the members take place in a single plane, the plane of the ecliptic; and they seem never to have taken thought to look up or down from that level in search of an extraneous motive power acting transversely. The universe of Newton's conception lacked the dynamical dimension of THICKNESS.

I trust that I have now said enough to convince the unprejudiced reader that there is plenty of room for improvement in the theory of astronomy as now taught in the schools, and to prepare his mind to receive new impressions and reconsider past judgments.

When, at the age of twenty-three, as the story goes, Newton saw the apple fall, the thought that occurred to him was not single but duplex. One phase of it was, Does the force of gravitation extend as far as from the earth to the moon?, and the other, If it does so extend, then why does the moon not fall, but preserve its uniform distance from age to age? Doubtless thousands of men before him had asked themselves the same queries, but given them up as unanswerable conundrums. Newton, however, did not. It occurred to him that, supposing the moon at every mathematical point of her orbit to be directed, with undiminishing velocity, tangentially forward, she might still fall like the apple, but fall no farther than just from the line of the tangent to the rim of the orbit. The first time he made his calculation the result was so far out that he gave up his hypothesis as unsound, and charged his labors to profit and loss. Some years later, however, as luck would have it, one of the chief data of 
fact upon which he had relied, namely, the length of a terrestrial degree, was found to be erroneous, and when this corrected quantity was incorporated in his earlier calculation the result came out satisfactory. His chief doctrines are:

1. Every body continues inits state of rest or of uniform motion in a straight line, unless it be compelled by impressed force to change that state.

2. Change of motion is proportional to the impressed force, and takes place in the direction of the straight line in which the force acts.

3. To every action there is always an equal and contrary reaction, or the mutual actions of any two bodies are always equal and oppositely directed.

His law of gravitation is: Particles of matter attract each other directly as the product of their masses and inversely as the square of the intervening distance.

By way of a supplement to these laws, I quote a passage from Young's work (Gen'l Astr., Art. 421) :

Newton was not satisfied with merely showing that the principal motions of the planets and the moon could be explained by the law of gravitation; but he went on to investigate the converse problem, and to determine what must be the motions necessary under that law. He found that the orbit of a body moving around a central mass is not of necessity a circle, or even a nearly circular ellipse like the planetary orbits, but that it may be a conic section of any eccentricity whatever-a circle, ellipse, parabola, or even an hyperbola, but it must be a conic.

In investigating the laws of circulating bodies, it has been customary from the time of Newton to take, as the typical case, that of the earth and the moon, and from that to generalize as to all. Let us follow their example; but let us first seek to familiarize ourselves with the nature and the difficulties of our problem.

Most people imagine that it is an easy matter to draw a perfectly straight line. Such a thing is not possible for any human being, and even if it were accidentally accomplished, there would be no available standard by which 
the fact could be attested. It is said that the "flattest piece of metal in existence, though fashioned with great care, is not flatter than the rotundity of the moon. The curvature of the ocean, level as it may seem to us when looking directly down upon it, slopes away from its tangent about eight inches in the first mile. Compare this now with the curvature of the moon's orbit, which, according to mathematical calculation, swerves from its tangent only $1 / 10$ of an inch in a mile, or .0535 inches in one second of time, during which it travels 3350 feet.

The mean distance of the moon from the earth is 238,840 miles, according to Young. Astronomers have attempted to explain how it got there, saying it was originally a part of the earth and by their mutual attraction has been gradually forced out to its present position; others, that it came from a distance, and when it approached near enough, was lassoed by the earth's attraction; and Newton himself, that the Creator placed it in position.

Now, considered merely as an isolated fact, it would make very little difference to us whether the moon were a few miles further in or further out; but it makes all the difference in the world when this distance is taken in connection with the velocity of the moon in her orbit. For the feasibility of the Newtonian hypothesis presupposes the precisest, undeviating correspondence between the length of the space fallen through by the moon in one second of time and the rate of her tangential velocity per second, else must she fall to, or escape from, the earth. This point may be made clearer by a reference to Figure 1, copied from Sir Oliver Lodge's book, Pioneers of Science (p. 171), with the text accompanying it:

Now consider circular motion in the same way, say a ball whirled round by a string.

Attending to the body at $\mathrm{O}$, it is for an instant moving towards $\mathrm{A}$, and if no force acted it would get to $\mathrm{A}$ in a time which, for brevity, we may call a second. But a force, the pull of the string, is continually drawing it towards $S$, and so it really finds itself at $\mathrm{P}$, having described the circular arc OP, which may be considered to be compounded of, and analyzable into the rectilin- 


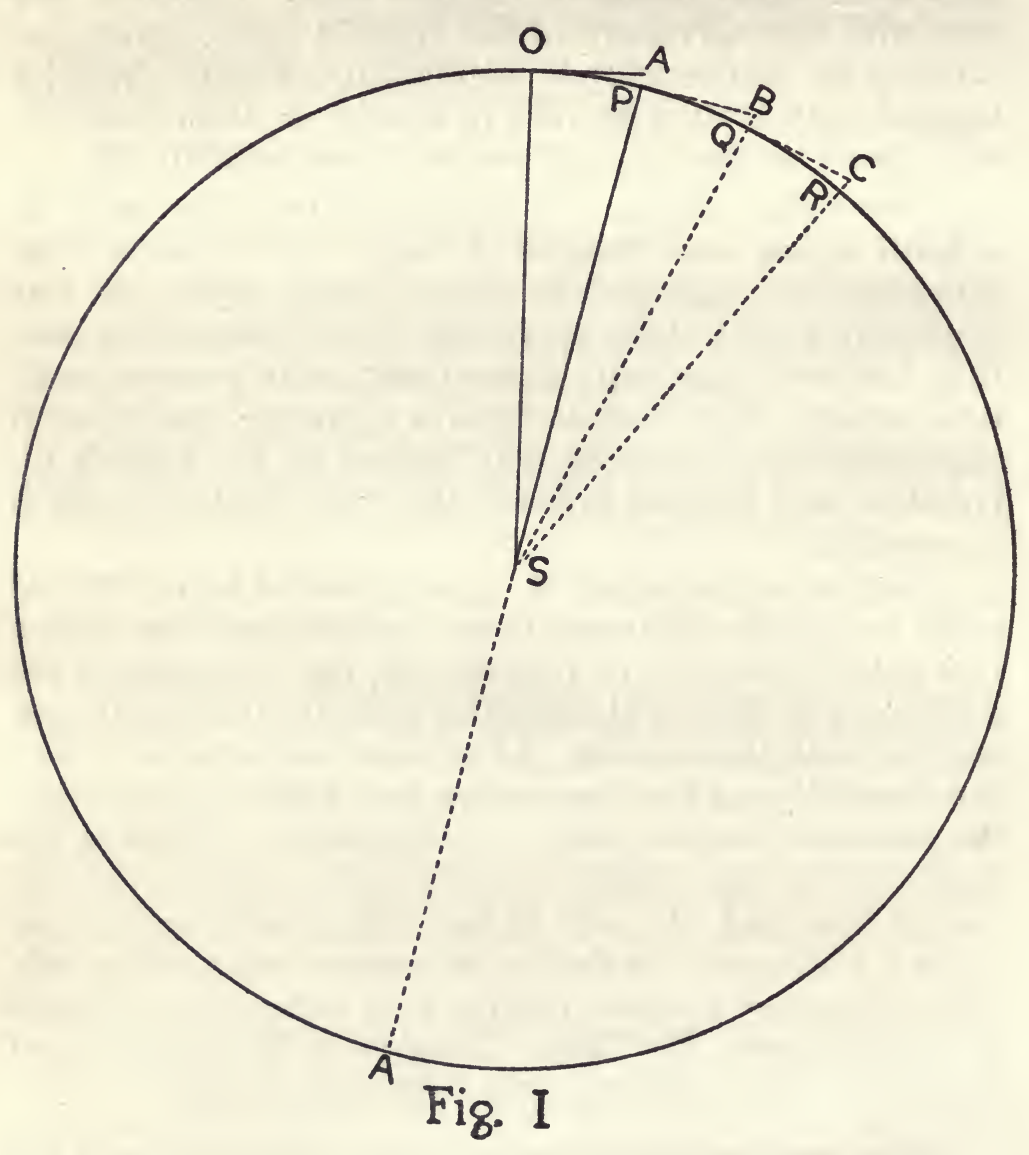


ear motion $\mathrm{OA}$ and the drop AP. At $\mathrm{P}$ it is for an instant moving towards $B$, and the same process therefore carries it to $Q$; in the third second it gets to $\mathrm{R}$; and so on: always falling, so to speak, from its natural rectilinear path, towards the centre, but never getting any nearer to the centre.

The force with which it has thus to be constantly pulled in towards the centre, or, which is the same thing, the force with which it is tugging at whatever constraint it is that holds it in, is $\frac{\mathrm{m} \mathrm{v}^{2}}{\mathrm{r}}$; where $m$ is the mass of the particle, $v$ its velocity, and $r$ the radius of its circle of movement. This is the formula first given by Huyghens for centrifugal force.

But suppose that, for any reason whatsoever, as by etheric or meteoric resistance, the moon's momentum (which, may the reader bear in mind, remains, according to Newtonian theory, altogether unexplained) should not avail to carry her clear to A, but only to some point by ever so little short of it, then the moon would inevitably sink at $\mathrm{P}$ within the line of her orbit, that is, nearer to the earth, where the latter's attraction would become even greater, absolutely and relatively, and consequently, during the succeeding second, overmatch the tangential velocity still more; a process which could not be stayed and would inevitably rapidly precipitate the moon upon our heads. On the other hand, suppose the tangential velocity to be ever so slightly excessive, so that the moon in its initial second of time could not at $\mathrm{P}$ drop clear to the orbital line, then would the earth's attraction, relatively and absolutely, acceleratingly decrease more and more, and the moon would consequently escape irrecoverably into space.

So precarious an arrangement as this surely cannot be the cosmic fact! Newton, with his hypothesis of divine agency, had a great tactical advantage over the proponents of spontaneous motions, who, no doubt, imagine that their hypothesis covers the requirements as responsively as his. But they are vastly mistaken. Were Newton here to answer us, he could consistently retort that the Creator is omnipotent; that it was within His power to place the moon just where He pleased; that He could at 
will fling it even more accurately than any mathematician could compute its proper course, and that He could impress upon it such velocity as He listed. Moreover, Newton might add, the Divinity still watches over His universe and by His mere fiat can readjust it, when and where needed, just as effectually as when $\mathrm{He}$ created it. Do I hear any astronomer make like claims of conscious design on the part of inanimate nature? (v. pp. 3, 7 ante.)

Now, in the solar system there are eight major planets, some twenty-five satellites, and more than 700 asteroids, all of whose orbits are very much larger than that of the moon, and whose trajectories consequently are infinitely straighter. Are we to believe that these, too, have uncaused motions, that these motions are all miraculously tangential, and that their speeds are magically accommodated by mere accident to the strength of their several central forces?

Again, returning to Figure 1, suppose the moon to have initially left the point $O$ with a velocity capable of carrying her in one second, under the first law of motion, to the point $\mathrm{A}$, would she on arriving at the point $\mathrm{P}$ (the earth's gravity having meanwhile acted) possess the momentum necessary to carry her in the succeeding second all the way to $\mathrm{B}$ ( $\mathrm{AB}$ being taken as equal to $\mathrm{OA}$ )? Most certainly not. For, as every tyro in mathematies knows, any are of any conic section is shorter than its tangent, $\mathrm{OP}$ is therefore shorter than $\mathrm{OA}$, and the moon's velocity at $\mathrm{P}$ cannot possibly be as great as when she left the original starting point. It matters not, therefore, how accurate the initial adjustment may be hypothesized, its perfection perishes in the very next moment.

By way of an attempt to parry this difficulty, Newtonians have succeeded in persuading themselves that mathematical exactness between the velocity of the tangential motion and the force of gravity is not vital to the practicability of their conception. They tell us, with every show of confidence in their words and manner, that if the velocity bears to gravity a certain ratio, the resulting orbit will be a circle; that if this velocity be exceeded, 
the orbit will be a parabola or hyperbola; and that if the velocity be deficient, the result will be an ellipse, broad or narrow, according to the degree of such deficiency; but that in no event can the circulating body fall in. When you come to scrutinize their argument closely, however, you will discover that they assume the tangentiality of direction at each and every point of the moon's orbit as a pure gratuity, for which they neither vouchsafe explanation, nor even concede the need of it. Newton, indeed, escaped this difficulty by boldly invoking Divine ordination in the placing of the moon exactly on the line of her predestined orbit, imparting to her the precise velocity requisite to offset the earth's gravity, and establishing with infinitesimal nicety her tangential motion; but this Aid, we have seen, his followers voluntarily renounce, non constat they offer us nothing to take its place. Be the orbit the shape of any conic section it may, Newtonian theory is compelled to postulate motion tangential to it at every point from the very beginning, else the body in question must, even in accordance with Newtonian premises, either escape from the central attraction or fall toward it, as the case may be. Further, even granting tangentiality of motion as a "natural" phenomenon "demanding explanation only as mere existence does", the most trifling excess of velocity, or the least diminution in the force of the centripetal attraction, or the slightest outward displacement of the body would, under the premises, mean its inevitable escape into space; while the antitheses of these, or of any one of them, would mean the prompt collapse of the whole system. I reiterate, Newtonian theory throws absolutely no light whatever on how the moon acquired her tangential motion in the outset, how she preserves it, how she acquired her original velocity, how this was, and remains, so miraculously adapted to the intensity of the earth's gravity, or how all these remarkable adjustments managed to come into existence simultaneously, as the theory presupposes.

Let us reduce the argument to mathematical form: According both to theory and observation, the moon 
at perigee has always exactly the same momentum. Considering, then, two successive perigees, let $M$ represent the Moon's momentum at the time of the first perigee, and $M^{\prime}$ that which she has at the time of the second, and we have,

$$
M=M^{\prime}
$$

Again, by general consent the centripetal and centrifugal forces are equal, which fact we may express, for one lunation, thus :

$$
C=C^{\prime}
$$

But astronomers inform us that the momentum, $M$, of the moon supplies the centrifugal force, consequently, on this theory, the momentum of the moon at the second perigee must be $M-C^{\prime}$; substituting which in place of $M$ in the first equation, we get,

whence, and, since $C=C^{\prime}$

$$
\begin{aligned}
& M=M-C^{\prime} \\
& C^{\prime}=0 \\
& C=0
\end{aligned}
$$

Reductio ad absurdum: There are no central forces: gravitation is a myth (v. p. 17, ante).

Newtonians are by no means oblivious to the insecurity of the ground they here occupy, so they have added, by way of an alternative or makeweight, the fiction that celestial motions are "persistent". This idea they have borrowed from the so-called doctrine of conservation of energy. They have come to realize that Newton was too modest in asking merely for the gift of rectilinear motions by way of capital on which to run his cosmic system, and so they have added, for the sake of expediency, this new notion. In other words, they have gratuitously substituted in the Newtonian vocabulary the word persistent for inertia, an antonym. Now, our terrestrial experience offers us not a single example of motion not plainly traceable to a definite physical cause; so, to mark the difference, and as a sort of honorary distinction, they call the one sort "celestial" mechanics and the other, "terrestrial", exactly traversing, as it were, Drummond's Natural Law in the Spiritual World, by 
showing that natural law does not even rise to the height of the physical heavens!

A little sober reflection ought to convince anyone that attempts to solve the problems of the cosmos by adopting in the outset a group of "laws" transcending, and even contradicting, human experience, and, with these as a base, trying to harmonize celestial phenomena in other respects interpreted by mundane standards-must prove abortive. Natural law is consistent with itself from the greatest to the least; pervert one part of it and you involve yourself in an endless maze of error. Were the matter less serious, it would be amusing to note the airs assumed by astronomers at having "discovered" this supposed departure of celestial from terrestrial mechanics-this inconsistency of Dame Nature, the chief offender of her sex. Thus we find Miss Mary Agnes Clerke the distinguished historian of astronomy (Modern Cosmogonies, p. 10) writing naïvely: Kepler's ignorance of the laws of motion precluded him from the conception of velocities persistent in themselves, and merely deflected from straight lines into curved paths by a constant central pull." Let it once be contended that mechanical effects, such as the rectilinear motions of stars and planets undoubtedly are, exist independently of physical causation, and you commit four grave follies; (1) You deprive physical science of the only ground she has to stand upon, namely, the law of physical cause and effect, the sole key to the interpretation of nature; (2) You throw wide the door to the perpetration of all sorts of other pious frauds; for if Nature departs from her laws in one case, why may she not do so in any other, according to the exigency of theory or the whim of any of her would-be interpreters? (3) You commit yourself irrevocably to the hopeless task of trying to correlate facts with miracles, instead of facts with cognate facts; and (4) You require of the present order of Nature not only to regulate itself, but likewise to overcome and discipline the erratic elements left over or inherited from a previous state of chaos. 
Newton supposed that by argumentatively eliminating the resistance of the ether, postulating it to have existence but no body, he overcame the only obstacle to his inertial hypothesis. In this he was culpably in error. Take two pails precisely alike and provided with lids. Fill one with sand, letting the other go empty, and carry them together for a furlong. Being exactly alike in size, they are, of course, equally resisted by the atmosphere. Which arm, however, will tire first, that carrying the full pail, or the other one? The former, by all means! The moon, too, has a load resistance to overcome, besides having to buffet the medium it traverses. We shall presently find that this load amounts to the steady downward pull of 240 millions of millions of horses, more than a million times greater than the etheric resistance to the moon would amount to were that medium even as dense as our atmosphere at sea level. Talk about straining at gnats and swallowing camels! Returning again to the pails; would you say that by running the furlong instead of walking it, you would lessen the work done proportionally? Certainly not, and neither can the imaginary impulse that fired the moon on her course relieve her of the task of carrying her own dead weight. You cannot sophistrize gravity out of existence. To support the moon during the 29.5 days of her monthly journey from apogee to apogee and bring her back again to the same altitude, she must from some genuine source-not from empty imagination or word-juggling-draw just as much lifting power as would be required to counteract the earth's gravitation did she possess no initial tangential translation whatever.

Have you, my reader, any adequate idea of the elastic strength of the earth's attraction upon the moon? Do you realize that, unlike a rubber band, gravitational attraction never rots or wears out, never relaxes, and, moreover, even increases its tension the closer the bodies are brought together? It is not like a strand of twine, or a ship's cable, or a dog's tether, that remains in a state of laxity until called into play only when stretched to full 
length. No! it is unrelentingly pulling at the moon with all its might, striving to bring her down.

Now, it is not a difficult matter to determine by calculation the approximate strength of this attraction, once we know the respective masses of the two bodies and the intervening distance, all of which we do. Expressed one way, this attraction is equivalent to the full tensile strength of a solid steel cable 400 miles in diameter (say from Pittsburgh to New York) capable of sustaining a load of 40 tons to the square inch. Expressed in terms of power (supposing a horse able to sustain a ton against gravity), it is equal to that of 240 million, million horses. Allowing 100 square feet for each horse, it would require to stable them a four-story building covering the entire surface of the earth, not excepting the space taken up by the oceans! Visualize all this to your mind's eye, and imagine the horses, or a steam engine equal to them in power, located at the center of the earth and pulling upon such a cable, and ask yourself whether it seems to you reasonable that the unsupported moon, without any influx of energy whatsoever from without, can, unenfeebled, hold her own against it forever, as astronomers declare she does? To the casual observer the moon looks like a silver wafer pasted upon the sky; in reality it is a globe of earthy matter 2160 miles in diameter (as far as from New York to Santa Fé) with a mass nearly one-eightieth that of the earth itself. So far as we know or have reason to believe, it has remained exactly at its present mean height for at least 3000 years, and possibly has been there for as many hundreds of millions-all the time pulling against a strain of 240 trillions of untiring horses! The moon has no pillar to support it, no pulley to suspend it, no atmosphere to buoy it, no breeze to waft it, no screw to propel it, no engine to haul it,-yet still it stays up. Why? Whence comes the centrifugal force to keep driving it away from the earth with identically the same energy that the latter perpetually prompts it to fall? Where is the equivalent of that flinging motion of your hand that supplied the centrifugal force to the little ball in Sir Oliver's experiment? 
Nor is it much more difficult to determine the approximatehorse-power seconds of energy resident in the moon's "momentum". Assuming that body to be moving at the exact velocity of 3350 feet a second and that it is falling at the behest of the earth's attraction at the precise rate of one-nineteenth of an inch in the same space of time, we need only divide the fraction into the larger number and multiply the quotient by 240 trillions to get the answer desired. Remember, however, and again I say remember, that, according to Newton's theory, the moon has no way to recuperate lost energy; hence, when the energy of her momentum is used up in wrestling against the earth's attraction, that momentum is done for, for good. Now, by the conditions of our problem, the moon's momental (inertial) energy is constantly pitted against the earth's attraction, which is always fresh, can never be used up, and is uniformly self-renewing. Dividing, therefore, as we did above, 3350 feet, or its equivalent in inches, 40200 , by 1-19 we obtain the quantity 763,800 , which is the number of seconds that it should take the centripetal attraction to wear out the moon's momental energy completely. Raised to higher terms, this period amounts to 8.8 days, which is reasonably close to the time generally estimated that it would take the moon to fall to the earth if dropped from a state of absolute rest. This result agrees well with the rule that "projectiles fired horizontally reach the earth simultaneously with like objects dropped from the same height."

Again, does the moon really fall toward the earth as astronomers allege? It is admitted, on all hands, that its mean distance is quite, or at least very nearly, the same from month to month and from century to century. If it be correct to say that the moon is falling simply because it is continually diverging from the tangent of its orbit, it is no less correct to assert that the dome on the Capitol at Washington is falling, because it, too, is continually diverging from the tangent of the circle in which it revolves by reason of the earth's diurnal rotation; specifically, three inches per second. According to the doctrine of 
the conservation of energy, loss of "position" involves transformation of potential energy into kinetic, and for every unit of the former that disappears one of the latter must appear. If such be not the case in every instance, the law is no law at all, and must be relegated to the limbo of exploded errors. In one ordinary month there are about 2,551,400 seconds, or 708.7, hours, or 29.5306 days. If in one second of time, as alleged, the moon falls .0535 ins., it should develop kinetic energy equal to 240 million, million horse-power as long as the falling process continues, which, humanly speaking, means forever. What becomes of this energy, I ask? If nothing becomes of it and it passes out of existence in the moment of its creation, then must we not admit that there exists a creative energy in nature and also a way whereby energy can perish, neither of which propositions scientists now concede? If no potential energy, on the other hand, disappears and no kinetic energy appears, then there is no loss of "position" at all, the moon is not falling, and the whole Newtonian argument breaks down. Lastly, if the moon is not falling earthward, it is not construable as a "body falling in vacuo," nor, for the like reason, is the earth; and the elaborate theories of Newton's Tides and Darwin's Tidal Evolution are rendered wholly irrelevant. and prima facie absurd.

As you, of course, know, the moon travels around our earth, not in a perfect circle, but in an ellipse; of which latter the earth occupies the focus. The long axis of this ellipse is called the major-and the shorter the minor axis. Now, there is one way, and but one, whereby you can divide this orbit into exactly similar halves (at the same time severing the earth in half) and that is, by slitting it along the line of the major axis. Along one of these semi-orbits, the moon, proceeding from her perigee to her apogee, constantly decreases her velocity; while along the other, on her return journey, she correspondingly increases it. Now, the doctrine of "persistent velocities" holds that, in coming inward, the moon is endowed with the occult property of gaining, not merely in velocity, 
but in strength of persistency, or, what is the same thing, centrifugal force; whereas, in going outward, the contrary is alleged to be the case. So you see that, not even satisfied with demanding both uncaused rectilinear motions and gratuitously persistent velocities, we are asked to concede, further, that this persistency is variable; nor that, merely, but intelligently so! Moreover, every planet, every satellite, every asteroid, every comet, every circulating body throughout the universe of matter, we are asked to believe, is blessed with a similar occult power, each with a special rectilinear velocity, and a unique rate of increase and diminution, to suit the mathematical elements of its individual orbit-and the exigencies of Newtonian theory.

Thus, after long and arduous beating about the bush the astronomers have come back to the same point from which they started, flushed with pride at being able to tell us the illuminating news that they have discovered: that the planets got started of themselves, that they maintain and vary their speed by virtue of some secret unfathomable power, and that, in short, the whole business is quite as inexplicable as we at first found it to be! How sad to think that Kepler had to die in his "ignorance"!

Again, Newton concentrated his whole attention on the fact that the moon falls from her tangent a trifle of 1-19 inch per second, equivalent in a lunar month of 2,551, 400 seconds to scarcely two miles; but he and his followers have passed over with but scant attention the far greater variation in her altitude above the earth as between perigee and apogee, when, in only one-half the elapsed time, she rises the enormous amount of some 31 , 000 miles, or 130 feet per second as against the 1-19 inch that she falls by gravity! - and this in the very teeth of gravity! Later, on the return journey, she loses this again, falling, now, 30,000 times as fast as she falls by gravity, but by poor marksmanship, or something else less understandable, she misses the earth that is calling her, falls around it, and again away. As to the two miles 
she falls by gravity, they furthermore tell us, she doesn't fall this distance after all, because she comes no nearer! In the text quoted from Sir Oliver Lodge, accompanying Figure 1, he says: "Now consider circular motion in the same way, say a ball whirled round by a string." This habit of drawing a parallel between the case of the moon revolving around the earth and that of a ball being whirled about at the end of a string, with never a word as to the vital difference between the two phenomena, is nothing short of a pious fraud. It is a common occurrence in one's astronomical reading to come upon such expressions as, "the earth whirls the moon around," "the sun whirls his planets around", "the star whirls its companion around," quite as though these things were "most well-attested facts of human knowledge". As a matter of plain fact, the difference is the same as between a dead horse and a live one, between an automobile charged with gasoline and one that has run dry. When I whirl the ball around, I supply the power that stretches the string and overcomes the resistance of the atmosphere. When I turn to the earth, however, I can perceive nothing to correspond with $m y$ part in the scheme. I can readily see the analogy between the tension of the string and gravity, between the ball and the moon, and between the position of the earth with reference to the moon and that of my hand with reference to the ball; but as to the flinging motion of my arm, its counterpart is conspicuous by its absence.

It is scarcely to be doubted that Newton cast about long and anxiously for the physical foil to the earth's gravity which enables her, from century to century, to maintain her aloofness. Nor is it any less likely that every beginner in the study of astronomy passes through a period of wholesome skepticism more or less prolonged and harassing before he can bring himself to believe, if ever he really can believe, the fable that "no such foil is necessary." By dint of incessant iteration, however, coupled with their avowed "policy of discouraging discussion," our scientific institutions have managed to 
keep alive the fiction, invented by Newton himself, that the only drain upon the moon's store of momental energy lies in the possible resistance offered her by the ether, and that by merely positing this last as "imponderable" the whole theory is irrevocably clinched. Their argument runs that, in the case of the ball and string, the flinging motion is necessary not to stretch the string, but only to overcome the atmospheric resistance, and that, in the case of the moon, there being no atmospheric resistance whatsoever, no live flinging force at all is required.

One of my critics, a professor of astronomy in one of the oldest New England colleges and an uncompromising Newtonian, writes me: "The experiment you describe is demonstrably false. As long as the ball is in motion it requires no 'flinging force' to prevent its flying back to the hand, and were friction absent it would never stop in its motion. The answer to your question, 'Whence comes the centrifugal force to keep driving the moon away from the earth' is found in Newton's first law of motion.' Let us test this view by following out its logical deductions and see whether we can conscientiously subscribe to them.

Suppose, then, we choose an elastic string exactly a yard in length and capable of extension without rupture to double its length; and to one end of it let us attach a solid lead ball one pound in weight. Suppose, again, that I set the ball awhirling round my head until the string is stretched just to its limit. Now according to the Newtonian view, all the muscular energy I thus expend is wholly and exclusively absorbed in buffeting the air, and not so much as one infinitesimal part of it goes toward, or is required for, the tensing of the cord. If this view be really sound, then these propositions, which to my mind are reductios ad absurdum, necessarily follow:

1. If, while in the midst of the experiment, it were possible for me to step into, and exist, in a vacuum chamber, I could there cease my flinging motion altogether, and the ball would nevertheless continue to gyrate about my hand indefinitely. 
2. Were it possible for me to step into an aeroplane or etherplane and be carried out into free space, other things remaining equal, I should there merely need to start the ball awhirling and it would then go on revolving of its own accord forever.

3. If I should beat the solid ball into a thin sheet and fashion the same into a hollow ball ten times the diameter of the first, the air resistance would then be multiplied one hundred times and I should be obliged to exert a hundredfold as much muscular energy as in the first instance in order to keep the ball revolving at the same speed.

4. If a 64-1b. weight were substituted for the original one-pound, the air resistance would be increased only sixteen times; and, supposing me capable of managing so ponderous an object, I should, once the ball was in full swing, be obliged to exert myself only sixteen times as much as in the first example and less than a sixth as much as in the third.

5. Finally, were an object held absolutely stationary at any height above the earth and then let go, it should continue so indefinitely, because, (1) since it is, by hypothesis, not in motion it has no lateral resistance to overcome, and (2) inasmuch as, by allegation, no energy is used up in stretching the cord (resisting gravity), it cannot possibly fall. Carrying this particular example farther, let us imagine this otherwise stationary moon to possess at this instant a projectile radial velocity away from the earth exactly equal to the force of the earth's gravity (i. e. 1-19 in. a second). What, now, should be the natural result? Obviously, the body would neither fall nor rise during the first second of time, but would remain quite stationary, whereas in the succeeding second it would start falling just as though no such projectile movement had ever existed. This conclusion we can make sure of by a simple experiment: At the earth's surface gravity causes objects to fall 16 feet, instead of only 1-19 inch, in the first second. Now fling a bullet straight up 
into the air with this same initial velocity, and it will rise to the exact height of sixteen feet, there stop short, and, turning back, descend into your hand by the end of the second second. Suppose that instead of merely pitching the bullet upward, you should elect to fire it horizontally with a force capable of carrying it in a straight line to a level higher by sixteen feet than the gun's mouth, would you expect thereby to defeat gravity indefinitely, or would you not rather look for the bullet to return to the earth in a very brief space? What possible advantage can there be in employing the projectile force horizontally in preference to vertically, if the purpose be to escape gravity or hold it at bay? They tell us that for a bullet, fired vertically, to keep itself permanently aloof from the earth, it must be propelled with the initial, parabolic velocity of 6.9 miles a second, and that in that case it can not stop short of infinity. Yet here is the moon remaining perennially aloof, neither falling or receding! To all these difficulties Newtonians are secretly and poignantly alive, and this is why they have so recklessly perverted our language by rhyming inertia with persistent.

I respectfully submit to the combined intelligence of mankind that the cosmic phenomena demand a welling source of centrifugal force to correspond with the "flinging motion" in the experiment, and, further, that the factor of the resultant of the stellar attractions not only precisely fulfils this need, but requires, in any case, to be given a place in cosmological theory before the latter can fairly be adjudged complete. Is it not the better part of wisdom to utilize it constructively rather than forever to cravenly dodge and ignore it?

Another shallow artifice is the drawing of an analogy between the motion of a pendulum and that of a planet in its orbit, "the latter in one half of its orbit swinging in toward the sun under the influence of gravitation, and in the other half swinging away from the sun in consequence of the increased velocity thus gained'. Heretofore the Newtonian policy has steadily been to foster this fallacy, 
not to investigate it. Let us, for once, discuss it openly and frankly without let or hindrance.

In the first place, what is the construction of the pendulum, and what its principle of operation?

It consists of three essentials : (1) a point of support against gravity, (2) a pendent string or wire, and (3) a bob. When the latter is raised an are and then gently released, it descends to the lowest point and rises thence, without stopping, to an equal height (provided there be no air or other friction) on the other side. The principie is quite plain : gravity causes the bob's descent during one unit of time, and stops its ascent during the next unit. Without gravity acting, the bob would stay wherever it might be placed in the pendular circle. Now, where, I demand, is the source of this gravity of which mention has just been made-within or without the pendular circle? Without it, of course. But where is this source in the earth-moon system, for example? It is within at the very center of the "pendular" orbit. And where is the "point of support"? There is none.

Where, I ask the fair-minded reader, is, then, the analogy?

Let us see now if we cannot convert the illustration of the pendulum into a true parallel. Substitute in place of the supporting hook some imaginary fixed object in free space, and conceive to be attached to this an elastic cord in lieu of the original common string or wire; and at the loose end of this elastic cord, again, affix the erstwhile bob. Now conceive yourself as taking hold of the bob and pulling it away until the string is at high tension and then flinging the weight, tangentially, as hard as you have a mind to, and ask yourself whether it would continue revolving around the "fixed point" forever, maintaining throughout the initial tension on the cord?

Reverting to my theory: The earth constitutes the fixed point; her attraction for the moon, the elastic cord; the moon herself the $b o b$-and the stellar resultant the extraneous source of gravity. A true pendulum, this, throughout every degree of the circle. 
It is one of the ludicrous fictions of astronomers that the moon, having once gotten started in her tangent, is continually falling beyond the limits of the earth and therefore can never actually alight, but must continue circulating round and round the planet indefinitely. At perigee, for instance, the moon's velocity, they say, is such that it shoots the body so far beyond the earth, and with such force, as to fire her clear out to apogee, where gravity finally regains the upper hand and compels her return. On this return journey, they proceed, the moon is so strongly attracted toward the center of gravity of the earth as to cause her to acquire thereby so much momental velocity as to enable her to foil the attraction to which she owes that velocity, and at perigee to swing clear of the snare! To see how well the principle works out in terrestrial practice, try it out with a ball attached to a rubber string and see whether the contraction of the elastic will let the ball play any such tricks-without the flinging motion of your hand to aid. The notion that the moon can fall beyond the earth is in itself very silly, for, wherever it may be in its orbit, it is always over the very center of the earth and is being incessantly drawn toward that point.

To "elucidate" this matter in a way "intelligible to non-mathematical minds," Dr. Newcomb, in his Popular Astronomy (p. 77) says:

To the mathematician the passage from the gravitation of an apple to that of the moon is quite simple; but the non-mathematical reader may not, at first sight, see how the moon can be constantly falling towards the earth without ever becoming any nearer. The following illustration will make the matter clear: Any one can understand the law of falling bodies, by which a body falls sixteen feet the first second, three times that distance the next, five times the third, and so on. If, in place of falling, the body be projected horizontally, like a cannon-ball, for example, it will fall sixteen feet out of the straight line in which it is projected during the first second, three times that distance the next, and so on, the same as if dropped from a state of rest. In Figure 2, annexed, let A B represent a portion of the curved surface of the earth, and A D a straight line horizontal at $A$, or the line along which an observer at A would sight if he set a small telescope in a horizontal position. Then, owing to the curvature 


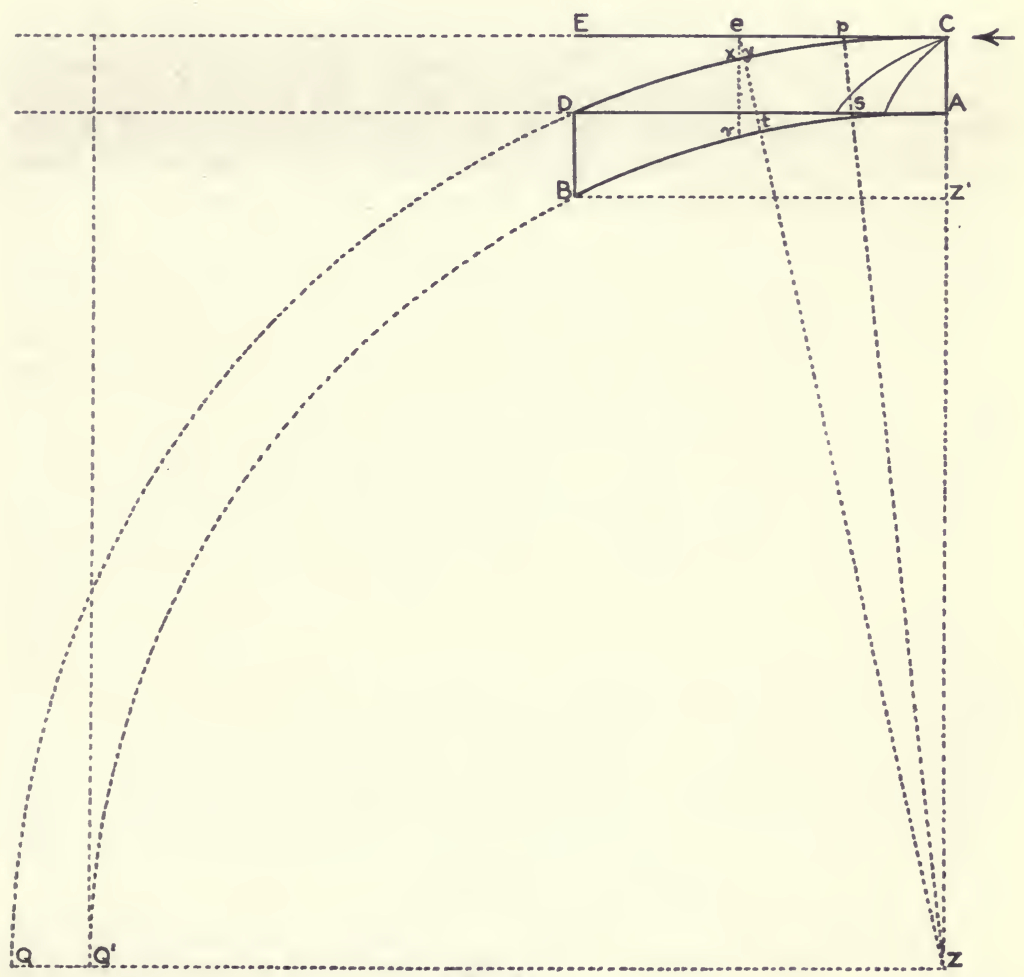

Fig. 2 
of the earth, the surface will fall away from this line of sight at the rate of about eight inches in the first mile, twenty-four inches more in the second mile, and so on. In five miles the fall will amount to sixteen feet. In ten miles, in addition to this sixteen feet, three times that amount will be added, and so on, the law being the same with that of a falling body. Now, let A C be a high steep mountain, from the summit of which a cannon-ball is fired in the horizontal direction $\mathrm{C} \mathrm{E}$. The greater the velocity with which the shot is fired, the farther it will go before it reaches the ground. Suppose, at length, that we should fire it with a velocity of five miles a second, and that it should meet with no resistance from the air. Suppose $e$ to be the point on the line five miles from C. Since it would reach this point in one second, it follows, from the law of falling bodies just cited, that it will have dropped sixteen feet below $e$. But we have just seen that the earth itself curves away sixteen feet at this distance. Hence, the shot is no nearer the earth than when it was fired. During the next second, while the ball would go to $\mathrm{E}$, it would fall fortyeight feet more, or sixty-four feet in all. But here, again, the earth has still been rounding off, so the distance D B is sixty-four feet. Hence, the ball is still no nearer the earth than when it was fired, although it has been dropping away from the line in which it was fired exactly like a falling body. Moreover, meeting with no resistance, it is still going on with undiminished velocity; and, just as it has been falling for two seconds without getting any nearer the earth, so it can get no nearer in the third second, nor in the fourth, nor in any subsequent second; but the earth will constantly curve away as fast as the ball can drop. Thus the latter will pass clear round the earth, and come back to the first point $C$, from which it started, in the direction of the arrow, without any loss of velocity. The time of revolution will be about an hour and twenty-four minutes, and the ball will thus keep on revolving round the earth in this space of time. In other words, the ball will be a satellite of the earth, just like the moon, only much nearer, and revolving much faster.

Our next step is to extend gravitation to other bodies than the earth. The planets move around the sun as the moon does around the earth, and must, therefore, be acted on by a force directed towards the sun. This force can be no other than the gravitation of the sun itself. A very simple calculation from Kepler's third law shows that the force with which each planet thus gravitates towards the sun is inversely as the square of the mean distance of the planet.

Only one more step is necessary. What sort of an orbit will a planet describe if acted on by a force directed towards the sun, and inversely as the square of the distance? A very simple demonstration will show that, no matter what the law of force, if 
it be constantly directed towards the sun, the radius-vector of the planet will sweep over equal areas in equal times. And, conversely, it cannot sweep over equal areas in equal times if the force acts in any other direction than that of the sun. Hence it follows, from Kepler's second law, that the force is directed towards the sun itself.

In transcribing the geometrical figure given by New. comb, I have taken the liberty of adding the dotted lines and using final letters of the alphabet to designate new points of reference. Let $\mathrm{Z}$, then, represent the center of the earth, Ze a radius vector of the moon (the projectile body), and let the line exv be drawn parallel with CAZ.

In this demonstration (for which, by the way, Newcomb is not primarily responsible) we have a concrete example of the difficulties that beset the path of those who persist in the attempt to compress into a scheme of but two dynamical dimensions the phenomena actually taking place in one of three. Observe, on the one hand, (1) that in Fig. 1 the circulating body (earth) is pictured-correctly-as forever falling toward the central attraction $\mathrm{S}$, and (2) that in the last paragraph of Newcomb's own statement he accepts this point, when he says: "Hence it follows, from Kepler's second law, that the force is directed toward the sun itself." On the other hand, however, when we turn to his diagram and the descriptive text accompanying it, we discover to our surprise that the path of the body, DB, instead of being pointed rarially toward Z as it honestly should, is cunningly drawn parallel with $\mathrm{CAZ}$, and points to nothing. Why does he assert that the earth is always falling sunward, but imply that the point of attraction races out along the line $\mathrm{ZQ}^{\prime} \mathrm{Q}$ pari passu with the projectile body and as if it will be at the end of the quadrant to greet the earth when she shall have descended to that point? Does he suppose the sun to possess an astral body which he can thus project out of his physical corpus and thereby prompt the planets to fall to places where he himself is not?

Now, for the sake of convenience, let us drop the example of the sun and the earth and substitute the more familiar one of the earth and the moon: 
Plainly, the moon instead of dropping to $x$, therefore, falls to $y$, and we have a repetition of the condition exhibited in Fig. 1, in which it was shown that the velocity of the moon, by the combination of the projectile force with the centripetal attraction, is slowed proportionately to that of an arc as compared with its tangent. That the two forces mentioned do not act cumulatively, but in opposition to each other, resulting in a logical diminution of speed, clearly appears from the direction of the line $p s \mathrm{Z}$, which depicts the mean direction of the action of the central force, and which visibly intersects the tangent at an acute angle. This loss of velocity, be it noted, is not in any sense attributable to the presence of a resisting medium, but is the inevitable and logical outcome of the principle of central attraction, and calls for the assignment of a substantial counteracting centrifugal force.

Suppose, for the sake of argument, that the moon really did fall acceleratively as Newcomb describes, then at the end of the first quadrant she will have gained a velocity equal to 3350 feet per second in addition to the "persistent" momentum with which she started out. Would not this constitute quite an embarrassment of riches? Furthermore, what shall be said of the contradictory statements: that the moon only falls two miles during the entire month; that she doesn't fall at all, since she perennially preserves her mean altitude; that she falls out to apogee and in to perigee 31,000 miles alternately; and, finally, that she falls not uniformly but acceleratively?

Suppose that you were called upon to run a hundred yards against time, starting from scratch and thence to and around a goal post and return; do you think you could make as good a showing and with no greater expenditure of energy as in a straightaway course of the same length? Of course you could not, nor could you find a normal boy old enough to know what it means to run such a race who would say you could. Astronomers, however, tell us that the moon can! Every month she starts from perigee, courses her way out to apogee, makes a wide detour, and 
returns to the starting point. At all points in her orbit 180 degrees apart, her compass direction is exactly reversed, showing that she must arrest all her motion eastward or westward, as the case may be, before she can acquire a new motion westward or eastward; yet month after month she has been doing this throughout the centuries, without, as alleged, drawing upon her stored energy to the extent of a single ounce, and without the loss of a shred of speed!

Imagine, if you please, some marvelous bird as large as the moon, whose wings could take hold upon the ether as those of our terrestrial birds do on the air, to approach us out of the depth of space, and to take up its course in the wake of the moon at precisely the latter's velocity; and when the race was full on, imagine, further, a hunter to shoot the bird dead in full flight. Would the slain bird and the inanimate moon be subject alike to the same laws of "celestial mechanics", and should we then possess two satellites in lieu of one? Would it, dead or alive, possess "persistent" motion by the magic spell of "entering an orbit", and be able, while living, to fold its wings and, without another effort, continue to fly forever in spite of its ponderous weight? Or could it, by an extra flap of its wings while in the throes of death, at the same time diverging outwardly from its tangent, thereby inaugurate for its carcass an unending flight into far distant space?

Another illustration drawn from the flight of birds may here be introduced by way of sharpening the distinction between the Newtonian theory of circulatory motions and my own. Instead of imagining one great bird, let us imagine two of them, the first as large as the moon and the other the size of the earth, and picture these as pursuing exactly parallel paths 238,000 miles (the mean distance of the moon) apart. According to Newton's first law of motion, were either of the birds unaccompanied by the other, once launched in motion it might placidly fold its wings, yet continue on its course, at unchanging velocity, forever. The fact of there being two of them, however, alters the case completely, for the gravitational 
attraction between them constantly tends to deflect them from their rectilinear courses, and to offset it the birds are obliged to resort to the use of their wings. At this stage, imagine the earth-bird to be struck fairly in the breast by a great comet with a violence, not only sufficient to slay it instantly, but also to bring it to a dead stop.

Up to this point, I take it, we are all in accord; but here we begin to disagree. According to the Newtonian view, the moon-bird may now fold its wings and cease its resistance to the earth-bird's attraction (which, of course, persists in spite of the creature's death), with the result of causing the former to circulate around the latter indefinitely; whereas I hold that in order to maintain its aloofness the living bird will have to exert its wingpower precisely as much as before. In a word, Newtonians while ostensibly representing the circulating body as traveling on the strength of its inertia, actually treat it as self-motored, i. e., as persistent, while I frankly face the truth and cite the Prime Resultant as the centrifugal agent.

As suggested before in the introductory chapter, it has been the habit of those who make a study of the heavens, from time immemorial, to anticipate finding a good deal of mystery and miracle intermingled with prosaic fact. The supposition that nebulæ rotate of themselves is a modern example of this primitive instinct. So is the crude belief in the spontaneity of celestial motions in general, and in the inherence in them of persistency as an abstract quality. Perhaps, however, the most typical instance of this superstitious streak in modern astronomers is their conception of the significance of the law of the conservation of moment of momentum. By observation and computation, the astronomers have discovered the fact (for such it is, subject to a modification to be explained later on) that the sum of the momenta, axial and orbital together, of any given system remains the same from year to year. (Upon this rock it was that the Nebular Hypothesis met final shipwreck, but of this in 
a later chapter). They find, for example, that the planets, in their revolutions around the sun, do not follow their courses without regard to what the others are doing, but, on the contrary, rhythmically swing in and out from their aphelion to perihelion in such a way that, no matter where a particular planet may be in its orbit, whether going fast at perihelion or slow at aphelion, the aggregate momentum of the group continues unchanged: just as though there were an understanding among them, or as if they were controlled by some guiding hand. Now, astronomers do not make any pretense of understanding this mystery; all they know is that it is a fact of observation. Unfortunately, however, they presume to build upon it, construing it not as the manifestation of an unknown physical cause but as a sort of teleological ordination transcending natural law.

It is upon this abstract, metaphysical, arbitrary, ununderstood "law" that the world of science has erected its proudest generalization, the doctrine of the conservation of energy, alias, the persistence of force, correlation of forces, transmutation of energy, mechanical equivalents etc.- high sounding phrases that all alike decree the death of Nature. In the following pages I shall peremptorily challenge this doctrine as absurd and unworkable, and a mill-stone around the neck of Science. "Beware of him of whom all men speak well," exhorted the ancient seer. Beware, say I, of this doctrine of conservation which all the world extols. I expect to prove to you, dear reader, that this mysterious conservation of moment of momentum is as simple a physical phenomenon in essence as the rotating of a top; also, that the molar movements of the heavenly bodies are not accomplished without the expenditure of power, but with a power, GRAVITATION, that is at once creative and coördinating.

To sum up, the planetary theory of Newton holds, in effect: That the planets are revolving around the sun by reason of certain original rectilinear motions having miraculously belonged to them under some pre-existing order of nature; that they travel through a medium pos- 
sessing objective existence without substance; that they can change their courses and bear loads without loss of momentum; that they have fortuitously happened to ally themselves to the same sun, to have hit upon approximately the same plane, to be traveling in the same direction, to have velocities bearing a fixed ratio to each other and to their solar distances, and, finally, that after hundreds of millions of years no accidents have happened to any of them, by meteoric collisions or otherwise, to disturb this precarious arrangement. It neglects to take into account the factor of the stellar attractions, the sun's movement in space, the age of the sun and earth, or the rotation of nebulæ. It does not clinch the law of gravitation, or explain the moon's secular acceleration, or solve the problem of the tides, or account for meteors, or explain the relationship between comets, asteroids, and planets, or give the physical basis for the phenomenon of conservation of moment of momentum. It harnesses bullets with horses, and pits finite moments against creative gravity. It pretends that an are is as long as its tangent, that the moon is falling to the earth's limb instead of toward its center, that persistent is a synonym for inertial, and that the whole minus an infinite number of times all its parts is still equal to itself.

Then why, you may ask, has the theory held sway so long? The answer is simple and lies in the nature of man himself, who finds in faith of some kind intellectual as well as religious repose. Just as many honest souls believe in this or that theological dogma under the mistaken conviction that denial thereof involves a denial of the Christian's God, so do men believe in this inertial theory under the hallucination that to deny it is to sacrifice the great cosmic principle of Gravitation itself. $M y$ system calls for no such sacrifice. 


\section{IV}

\section{THE PRIME RESULTANT}

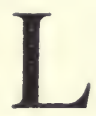

APLACE was right in assuming that the numberless concordances exhibited in our planetary system cannot be the results of blind chance. So far as the principles of the Newtonian system go, the planets are restricted only to orbits having the form of conic sections. These orbits might be as elongated as those of comets, or as inclined as the earth's axis, or possess any other eccentricity or tilt you please. Some might revolve clockwise, others contra-clockwise, others, still, straight up and down, backward or forward, through the celestial poles, for all the light Newtonian theories throw upon the matter. The fact, therefore, that the planets do not revolve thus indiscriminately, but conspicuously seek the same plane, revolve in the same direction, rotate on their axes in the same sense, and describe near-circle orbits, is decidedly too striking and significant to be dismissed as mere coincidence, and demands more than a teleological explanation. Indeed, what safe confidence can be placed in any cosmic theory that fails, as Newton's confessedly does, to clear up these remarkable concordances as one of its primary tasks and tests?

It is as plain as the noonday sun that the explanation we are groping for is a dynamical one, one, too, sufficiently comprehensive and general to compass in its mighty grasp the whole length and breadth of the system, so as to ensure that the effects it brings about may be universally consistent. The new factor, moreover, must 
allow as well for the exceptions to the general rule: it must be able to account, differentially, for the elongated orbits of comets as satisfactorily as for the rotundity of those of the planets; for the high inclinations of the asteroidal orbits as for the narrowness of the zodiac; for the rare cases of retrograde motion as for the vastly more numerous direct.

Doubtless the reader is familiar with the phenomenon of the miniature whirlpool that forms on the surface of a basin of water while escaping through a central outlet in the bottom. Next the circumference the rotation, as you know, is relatively slow, but it increases rapidly toward the center, where often a hollow cone is formed round which the water revolves with high speed. Do you know the reason for this? It is because the equilibrium of the water, as a whole, is constantly being unsettled by the falling away of its support, and the act of gyration follows as an automatic effort at a general balancing. If the basin were very deep and the process sufficiently prolonged, you might perhaps find it worth while to investigate the law of gyration by sprinkling some sawdust on the surface and timing the turns at different distances from the axis of rotation. I can tell you the answer, namely, the cubes of the distances are as the squares of the periodic times.

This phenomenon has several curious counterparts in nature-in the ocean, in the atmosphere, and in the skies. Thus Sir John Murray (The Ocean, p. 198) says: "The Michael Sars Expedition of 1910 measured tidal currents in the open ocean down to a depth of 400 fathoms. It was found that the currents at 274 fathoms ran in the opposite direction to that of the upper layers which again approached that of the currents at much greater depths. At certain moments the currents appear to be arranged in the form of a spiral staircase, the whole system turning in clockwise direction from top to bottom.' Again, Professor W. I. Milham in his standard work (Meteorology, p. 162), describes a similar peculiarity regarding the action of the atmospheric tides at 
the poles, even going so far as specifically to liken it to the "behavior of water in a wash-bowl while escaping through a central vent."

Now, if you will, slip on your wings of imagination and fly with me to a point in the heavens where we can obtain a good bird's eye view of our solar system spread broadside below us. But let us not view it, as customarily, from the north, but from the south side of the ecliptic, for there is a good reason which shall presently appear. Note the enormous size of the system. The sun in the focus is himself so huge that from his center to his circumference is almost 200,000 miles greater than from the earth to the moon. Yet that immense body, if viewed from Neptune, his outermost planet, 2,800,000,000 miles away, would barely show a disc to the naked eye. Suppose Uranus, whose distance is $1,800,000,000$ miles, to be directly opposite Neptune, on the other side of the sun, and then imagine a giant sun big enough to fill in the gap from one planet to the other. Retire now in spirit to the distance of the nearest star, Alpha Centauri, some thirty million, million miles away, then will this giant sun of our imagination appear to you almost exactly of the same size as would the real sun to an inhabitant of Neptune!

We see then that, scattered as the members of our system may be, and minute as they are as compared with their separating distances, they are nevertheless next door neighbors when contrasted with the stars in general. It is because of this relative isolation that they are enenabled, or rather compelled, to organize themselves automatically into a family or unit, in deference to the coercion of the cencentrated stellar attractions.

Universal gravitation I accept as an objective reality in all its plenary implication. If this force extends from the earth to the moon, from both of them to the sun, from the sun to Neptune, without sagging of the law of the inverse square, I contend that there remains no logical ground for doubting that it extends with unimpaired virtue throughout the visible universe to its outermost confines. If in this opinion I am in error, then the adjective 
universal has no significance in the phrase, and requires to be expunged. On the other hand, if I am in the right, it means that in failing to take into account the paramount dynamical factor in the universe-the stellar resultant-the current theories of astronomy cannot possibly be sound and, cost what it will, must be ruthlessly demolished to make way for an orderly reconstruction from the ground up.

Suppose only two bodies to exist in space, at rest, and separated from each other by a finite distance, they would then inevitably approach each other in a direct line and finally meet. Supposing the law of equilibrium to apply, namely, that freely moving bodies seek their lowest center of gravity; the direction of the axes of the two bodies would in that case point each other out in the sky until they met. Suppose, now, we add a third body and call them A, B and C, all of the same size, starting from rest, and so placed as to mark the points of an equilateral triangle. In this case the axes of the bodies would not point to each other, but would instead aim at the blank point being the mathematical center of the triangle, where eventually they would all simultaneously collide.

Now, we do not live in a universe of two or three bodies, but in one of many millions; not in one of two dimensions, but of three. How can we ever expect, or even hope, to be able to orientate ourselves in so complex a maze as this! Simply enough. All we need do is to reinstate the law of equilibrium, to wit, that all free bodies, whether in a vacuum or out of it, whether falling or in suspension, seek their lowest center of gravity. If we view the earth in this light, we must be blind indeed if we cannot discern that the reason its axis is "fixed" and points so steadily in one direction the year through, on whichever side of the sun it may happen to be, is because the northern hemisphere is heavier than the southern, or vice versa, and that the resultant of the stellar attractions is drawing our globe either due north or due south.

But how, you may ask, can we possibly tell which? I answer, by contemplation of the earth itself. Many at- 
tempts have been made from time to time to explain the great preponderance of continents in our northern hemisphere and their pronounced tendency to cluster around the north pole. No one needs to be told that rock is heavier than water, and where the contest is for the lowest place, the weightier substance will sink and cause the level of the lighter, liquid, to rise. Consider, also, that the longer a cause continues, the more its effects accumulate and thrust themselves into evidence. The earth has demonstrably been suspended over the abyss of space for millions of years, until, as in the case of a floral hanging-basket, its crust has, so to speak, protruded downward through the meshes. After this manner it is that mountain ranges are formed on so huge a scale. Like cakes of ice jamming against a pier, the hinder continents, or, often, the ocean beds grown irresistibly ponderous by the sedimentary accumulations of ages, press or lurch heavily forward, not only relegating the oceans to the rear (south), but crowding the land masses in front, bending, jamming, wrecking and crumpling them until, for the time being, further progress becomes stalled. Thus the earth's crust moves in gigantic billows and alternately rises above and sinks below the level of the sea. Most certainly, then, the northern hemisphere is the heavier, hence the under, and the earth is falling in the line of its axis northwardly. Logically, therefore, our school maps should be inverted, and it is my opinion that had they been hitherto so printed, this discovery would years ago have been anticipated. The Arctic regions are not the roof of the world, as they are often called, but the bottom of it.

It has long been one of the primary puzzles of geology as to why mountain ranges seemingly wait to be lifted up until the sedimentary rocks, of which they are mostly formed, attain their maximum thickness. Professor Joly, in his great work, Radioactivity and Geology (p. 97), dwells wonderingly on this singular freak of Nature's in seemingly waiting for her task to rise to maximum difficulty before exerting her powers, just as though 
she deliberately planned it thus in order to test the limits of her real strength; and in corroboration he quotes this from Dana :

A mountain range of the common type like that to which the Appalachians belong is made out of the sedimentary formation of a long preceding era; beds that were laid down conformably, and in succession, until they had reached the needed thickness; beds spreading over a region tens of thousands of square miles in area. The region over which sedimentary formations were in progress in order to make finally the Appalachian range reached from New York to Alabama and had a breadth of 100 to 200 miles, and the pile of horizontal beds along the middle was 40,000 feet in depth. The pile from the Wahsatch Mountains was 60,000 feet thick, according to King. The beds of the Appalachians were not laid down in a deep ocean, but in shallow waters where a gradual subsidence was in progress, and they at last, when ready for the genesis, lay in a trough 40,000 feet deep, filling the trough to the brim. It thus appears that epochs of mountain making have occurred only after intervals of quiet in the history of a continent. * * * Nor would the list of such crustal movements be complete save in the enumeration of every great range upon the earth.

Plainly, then, the formation of great mountain chains is analogous to the action of condensed moisture on the outside of an ice-cold pitcher. For a second or so, a small drop, after coursing down an inch or two, will often stop dead, held in its place by adhesion, until, being reinforced by a fresh drop following in its track, it will again start downward, perhaps to stop a second time and repeat the performance before attaining the bottom. So, before a mountain range can be lifted into place, a great dynamical agency must be accumulated for the work in hand. This Nature does gradually by laying one stratum upon the other on the bed of the ocean and incidentally eroding away the obstructing land in front out of which to form those strata, until the weaker becomes the stronger, or the lighter the heavier, and crowds its way onward toward the "bottom". Of course this process is exceedingly slow, and its results are normally very gradual, the strata bending rather than fracturing; but by no means is this always the case, for sudden slips are here just as bound to occur as landslips at the Panama Canal or iceslips in the Alpine glaciers. 
Thus we learn that the earth's general form, its topography, and its continental distribution and configuration, are determined by a tri-dimensional gravitational scheme, namely, (1) by the point of attraction connoted by its own center of gravity, whose endeavor it is to mould the planet into a perfect sphere; (2) by the plane of attraction, to-wit, the influence of the other members of the solar system, whose function it is to produce the tides in atmosphere and ocean, etc., and (3) by the third dynamical dimension, completing the "solid", being the joint or composite attraction of the outside universe that compels the earth, under the fundamental law of equilibrium, to keep lowering her center of gravity more and more by precipitating her heavier surface substances north-pole-ward. These three influences, then, all operating simultaneously, cannot but result in a deformation of the general contour of the earth; producing, as the most important effects besides those mentioned: (1) the squashing or flattening of the globe between the poles (much as a lump of soft putty behaves when set upon a shelf), an operation which, of course, implies (2) a corresponding enlargement of the planet's equatorial protuberance, heretofore erroneously ascribed to the running effect of the earth's axial rotation, (3) the special flattening of the northern hemisphere as compared with the southern, (4) the subsidence and partial submergence of the Antarctic continent, and (5) the "countersinking" of an arctic ocean bed by the shouldering of the continents against each other and the hunching up, as it were, of their backs.

In earlier eons, the migration of the continents northwardly was doubtless much faster, relatively, than it is to-day, for now they have become jammed, and hence much longer accumulations of depositions must precede every infinitesimal advance. Have you ever wondered how the Esquimaux came to settle in the Arctic wilds? Have you never marveled at the highly organized forms of life that inhabit those inhospitable regions? Do you think such life as that can have evolved there from prim- 
ordial forms? No! There is only one explanation that will serve, and that is, that the land which they and their forebears have inhabited from paleolithic days on, has not been the immovable platform for which they have all along mistaken it, but a land raft that imperceptibly has carried them through genial climates and latitudes to where they now find themselves.

Before proceeding to the more general problems of the solar system, let me emphatically warn the reader against inferring that the axis of the earth is pointing to the center of the universe. I do not mean to convey any such impression. An apple falling from the twig of the tree where you may be sitting as you read this, and one falling at the same time in the antipodes, though in absolute space moving in opposite directions, are really tending toward the same point, namely, the earth's center of gravity. But no one would think of arguing from this 'hat the earth's center is therefore the center of the unirerse! Mass for mass, proximate bodies dictate much more strongly than those more remote the course a celestial orb shall follow, and no two bodies are affected by the sum of attractions to the same extent, or impelled toward the same mathematical point in space. We know, furthermore, that all of the stars are in rapid movement, not only absolutely but relatively, for which reason the goal or focus of the attracting powers is forever rhythmically shifting about. When I assert, therefore, that the earth is headed toward a blank point in the heavens, I mean that the merging of all the attractions focussed upon her unite to coerce her movement, not only in a definite line but also to and through a definite, unmarked, mathematical point in that line. This line I call the Prime Resultant, signifying thereby the stellar resultant specifically pertaining to our solar system or any component member of it; and the point I denominate by the name Vertex.

Let it be thoroughly understood, however, that although, for the sake of convenience and brevity, I may speak of both these entities as if they were fixed in loca- 
tion, they are as a matter of fact continually shifting in position. This they do, not arbitrarily or capriciously, but rhythmically, and in strict amenity to gravitational law. All the stars are continuously tending together, and as they progress in their various courses it is inevitable that their composite attraction, varying as this does, not simply inversely as the distances, but as the squares of these distances, must likewise continuously undergo variation both as to locus and intensity. In short, our earth will never reach the end of her journey; wherever she may arrive, she will always be solicited toward some more distant point. Were she indeed this instant by fiat transported to her present Vertex, or gravitational goal, she could not there rest stationary, but would be obliged to resume her cosmic wanderings ad infinitum. In this process of translation we may discern some analogy to the circulation of the blood in the living animal; the systole and diastole of the heart, even, finding their counterparts in the gravitational contraction of the star and its reaction by explosion.

It is a habit with astronomers, repeating in their way the awkward method of geographers, to imagine themselves surveying the ecliptic from the north. According to their way of looking, the motions of the earth on its axis, of the planets around the sun, and of the moon around the earth, are all contra-clockwise (like a clock dial reflected from a mirror), whereas viewed from the south, as I advise, these motions all turn clockwise. It is well to get clearly in mind right at the very outset the fact that all of these motions run in the same direction. This is the rule throughout the system (with a very few minor exceptions among the satellites, which will be subsequently considered).

My object in viewing the ecliptic from the south instead of from the north is, however, of much greater significance than merely to aid the memory by substituting a right-handed motion in place of a left-handed one. In fact, it is for the same reason that I recommend the printing of terrestrial maps inverted, namely, to instill 
and cultivate the sense that we are falling, and that north means down. I wish the reader to realize as vividly as possible that in surveying our system from a point south of it he is looking down on the action of a whirlpool, physically as real as the surface of water emptying from a wash-bowl. By virtue of the suction of the Resultant, the weight is constantly slipping away, more at the center than elsewhere, necessitating continuous readjustment of the systemal balance. Due to its mobility and molecular attraction, the water in the basin reacts as a unit to central suction, and, in the same manner, the movability of the planets and their mutual gravitational attractions enable them to constitute themselves into a gravitational unit and sub-units, with similar power to readjust their common balance by a process which may be described as one of progressive equilibrium. There is the great whirlpool embracing the system as a whole, and there are also the eddies within it of Jupiter and his satellites, Saturn and his rings, and the rest. Or, if you like, you may think of our system as a prodigious clock, without visible springs, pinions, or hands, and with mere points to mark the various divisions of time, being driven by the force of its own fall. As the earth is a simple body conforming to the law of lowest center, so the earth and moon together form a compound body seeking their lowest common center of gravity, and so, too, do the sun and all the planets and their retinues summed together constitute a complex body, riding upon one common center of gravity. Where does the series end, or has it an end? May not our system be but a member of a system next greater, that of another larger still, and so on up to starstreams and the great whirling tire of the Milky Way itself, welding the entire universe, be it finite or infinite, into a single correlated Whole, all under the unifying, organic law of BALANCE?

Though they are scarcely necessary to prove the issue, it may be well before leaving this subject to point out two other considerations why and how the planets are thus caused to gyrate by the action of the Resultant. 
Were the members of our system actuated only by their mutual attractions, it is clear that they would speedily precipitate themselves into the sun. On the other hand, were they released from this bond and subjected solely to the action of the Resultant, they would necessarily fall in sheer lines. Inasmuch, now, as they are acted upon by both of these forces jointly, it follows that they can do neither the one thing nor the other singly, but must automatically effect a compromise between them. A little reflection will show that the only permanent compromise arrangement conceivable is, that the impetus of the cosmic fall of the planets, on the one hand, and their mutual attractions on the other, shall reciprocally balance, or, in other words, that the centrifugal force shall equal the centripetal. The second consideration, as already adverted to in Chap. II, is, that the Prime Resultant is not a mathematical line but a vast sheaf of rays of attraction which, in combination, operate at torsion. Returning now to our former (implied) equation (v. p. 78):

$$
M-C+C^{\prime}=M^{\prime}
$$

we perceive that $C$ and $C^{\prime}$ are no longer mere nullities, but immense real quantities, of a creative nature; though they cancel each other, indeed, in the shape of the work done in whirling about the huge boulders we call planets.

If this conception of mine, namely, that the Prime Resultant is the mother of the centrifugal forces, be sound, then we do not need to search any further for the explanation of those classical problems of celestial mechanics: the secular acceleration of the moon's mean motion, the rotations of the lines of apsides, and the anomaly of Mercury's perihelion motion; for all of these alike are soluble on the hypothesis that the source of the centrifugal power is accelerative, in unison with the law of falling bodies. The extreme importance which Newtonians have been attaching to these enigmas, especially to that concerning the moon, may be gleaned from the fact that ever since the time of Newton, when Halley first discovered the anomaly, scarcely one of the first rank of mathematical astronomers has failed to give it preced- 
ence over all other theoretical problems. The one great, obsessing aim has steadily remained, not to accept it as a friendly guide-post to new truth, but as an obstruction in the road which must be calculated out of existence at any cost. Fortunately for the salvation of the science, it is now all but universally conceded that "this deviatiou of the moon from its calculated path cannot be accounted for by any gravitational cause" (i. e., consistently with Newtonian theory) and numerous conjectures are being improvised to account for it on its own merits; none of which, however, possess any correlating virtues. Doctor See, for example, claims to explain it by postulating a "gravitational, screening effect" by the periodical eclipsing of the moon by the earth, and vice versa; thus obviously not only cutting this phenomenon off from all possible relation to others, like the anomaly of Mercury and the rotation of the apsides, but dangerously impugning the law of mass by implying a similar screening of the rear hemisphere of every planet by its fellow.

Had astronomers been as slovenly in their character as mathematicians as they have been in their character as theorists, this delicate deviation in the lunar motion would long since have been brushed aside and forgotten. For, as theorists, they have admitted terms which should have been excluded, while as mathematicians they have taken the utmost pains to incorporate even the most insignificant of genuine factors. The worst offender of all was Laplace, who in excess of zeal introduced the eccentricity of the earth's orbit as a cause, when under any view of the case, whether Newtonian or mine, this eccentricity should properly be treated as a concomitant effect of the underlying dynamical agencies at work, whether these latter be known, or simply inferred. As for the amazing and highly creditable care exercised by the mathematicians, let these extracts show:

Notwithstanding the accuracy of Mayer's tables, an irregularity had been discovered by observation which was not indicated by the theory of gravity. Halley and other astronomers had placed it beyond a doubt that the moon performed her monthly revolutions round the earth in a shorter time than formerly. 
This acceleration of the moon, as it was called, amounted to nearly ten seconds in a century, and various hypotheses were framed to account for it. The most plausible of these was, that all space was filled with an ethereal medium which opposed such a resistance to the motions of the planets, that the force which kept them in their orbit would gradually overpower their diminished velocity, and thus shorten their period round the central body. This hypothesis was supported by Euler, and by the abettors of the undulatory theory, who required the existence of a medium for the propagation of light, and it was adopted with equal eagerness by another class of theorists, who saw in the acceleration of the celestial motions the process by which the Almighty was to destroy the solar system, by precipitating the secondary planets upon their primaries, and the primary planets upon the sun. Laplace admitted the sufficiency of the hypothesis, but as he saw no reason for admitting the existence of a resisting medium, he did not consider himself warranted in adopting such an hypothesis till it was found that gravitation was incapable of accounting for the fact (Brewster, M. of N., p. 307).

The Academy of Science proposed the moon's acceleration as the subject of their prize for I770. Euler gained it, but came to the conclusion that it was not produced by the force of gravity. The same subject was again proposed in 1772 , and the prize was divided between Euler and Lagrange. Euler ascribed the acceleration to a resisting medium, and Lagrange evaded the difficulty. The prize was again offered in 1774, and was gained by Lagrange, and he now doubted the existence of the inequality. It was under these circumstances that Laplace took up the subject and found the acceleration to be ten seconds during a century, and to be caused by the eccentricity of the earth's orbit.

The curious subject of the moon's acceleration has recently excited much interest in consequence of Prof. Adams having recently proposed an important correction upon the theory of Laplace, by which the secular acceleration was reduced to 6.II". * * * The difference between the results of Mr. Adams (confirmed by Delaunay), if correct, and those obtained from ancient eclipses, is very remarkable, and indicates the operation of some cause which remains to be discovered (ibid., p. 3IO).

$\mathrm{Mr}$. Sterner and I in our investigation of the theory of the moon's motion, have probably occupied altogether about 8 or 9000 hours. There were about 13,000 multiplications of series made, containing some 400,000 separate products; the whole of the work required the writing of between 4 and 5 million of digits and plus and minus signs. Although the problem now completed constitutes by far the longer part of the whole, much remains to be done before it is advisable to proceed to the construction of the tables. (Prof. Ernest W. Brown). 
If the earnest reader will now be good enough to close his eyes for a minute or two and clearly picture to himself our solar system-segregated-falling-and having focussed upon it the thousand millions of stellar gravitational strands (like so many guy ropes in the hands of workmen setting in place a corner-stone), all, in a certain sense, pulling together, and yet, also, straining at cross purposes, with an effect of ceaseless unwinding; and if he will then rehearse the list of multiple planetary "concordances" enumerated by Brewster and Young as "transcending gravitational explanation," he can scarcely fail to perceive, as if by intuition, the physical necessity for the following actualities:

1. The vorticle gyration of the system as a whole, or unit.

2. Such gyration on the part of each subordinate system. tion.

3. Gyration, generally speaking, in the same direc-

4. Constant tendency and approximation toward orbital rotundity.

5. Similar tendency and approximation to one plane, partly because of centrifugalization, but mainly because of the constant struggle toward ensemble equilibrium.

6. Impingement of vortices, resulting in erratic evolutions at their margins of interference.

7. Elongated orbits and high inclinations as indicative of comparatively recent advent into our balanced order.

8. Gyroscopic reaction of the solar system, connoting for the sum a curvilinear orbit and betokening likewise the identity of his period of revolution with that of the precession of the equinoxes.

9. Conservation of the moment of momentum.

10. Tendency to axial rotations in the same sense sa the orbital revolutions. 
11. Commensurateness of orbits, conformably with the laws of equilibrium and of falling bodies combined.

12. Slow, gradual acceleration of the orbital times of revolution in accordance with the law of falling bodies, the evidence thereof being distinctly perceptible in the phenomena of the excessive progression of Mercury's perihelion, the rotation of the apses and the acceleration of the moon's mean motion.

Thus does the introduction of the Prime Resultant into astronomical theory justify its entrance by explaining, dynamically, all those numerous and amazing concordances that Laplace sought to explain by postulating the monstrosity of a self-rotating nebula. Indeed, it explains the rotation of all the nebulæ just as effectively, for these, too, are gravitational units. So are all genuine star clusters. Even the Milky Way itself is a gravitational unit, composed though it is of billions of separate stars, seeking its equilibrium with respect to the stellar resultant playing upon it from many distant universes like it, so far distant, indeed, that their light dies out before it can reach us. The facts are not susceptible of observational proof, but to my vision the Galaxy is nothing else than a dise of starry globes extending indefinitely beyond our keenest telescope, and revolving, like our solar system, about an axis passing through its poles.

\section{Retrograde Motions}

But what of the retrograde motions? These I divide into three classes: those of comets, those of asteroids, and those of satellites.

The first of these classes involves the solution of the nature and origin of the comets. These bodies, as I shall later explain more fully, are fragments cast off by exploded stars and enter our system as fugitives from their own land seeking an asylum in ours. When they first make their appearance, they exhibit certain eccentricities that proclaim them at once as strangers. For one thing, their orbits are very elongated, with diverse inclinations, 
and, for another, they are just as likely to have retrograde as direct rotations. It goes without saying that such erratic visitors cannot fail to disturb the balance of our system to some extent, and themselves be reacted upon in turn; just as the throwing of a basin of water into the sea theoretically affects the general level and the mutual relations of all the drops in it. From the instant of its haphazard injection into our system, the comet feels the influence of the gravitational current or whirlpool in which the planets are rotating, and immediately begins the process of accommodating itself to the balanced order of our regime. This process is vastly prolonged, but it is sure and steady, and it results finally in rounding out the comet's orbit, in reducing its inclination, and often in converting its retrograde motion, if such it had at the start, into a direct one. In short, the alien becomes a fullfledged citizen. Thus I deduce that many (not all) of the asteroids are simply domesticated comets, and that their departure from planetary regularity in the matters of retrogression and orbital eccentricity and inclination are just so many survivals of their preceding cometary phase, which time will largely wear away.

There is, however, a second cause for retrograde motions, and this applies more particularly to satellites. The three or four instances among these bodies exhibiting this peculiarity, it will be remembered, are the outermost members of their respective systems. Now, between the eddy of Saturn's group and that of the Jovian group, for example, there is a point or line where the circumference of the eddies may be supposed to impinge or "interfere," whirling there in opposite directions. This region, however, is not a well defined line but a rather nebulous zone, so that a body which for any reason has become entangled in it halts, as it were, between two allegiances, undecided whether to attach itself finally to Saturn's system or to Jupiter's, thereby giving rise to gyratory motions of a hybrid nature. In addition to this conflict between the minor vortices we must also not neglect to take into account the fact that the farther a satellite stands 
from its primary the more amenable does it become to the domination of the main vortex and the more apt it is to graduate into a planet.

Here, too, we have the complete physical explanation of the conservation of the moment of momentum, which, as shown in the preceding chapter, has heretofore been explained teleogically. The Prime Resultant is, as everyone can readily perceive, a physical force that compasses the entire system, so that all changes of momentum are accelerated throughout in like degree, thus all but concealing the fact of change. In other words, the yardstick with which we here measure shrinks and expands synchronously and commensurately with what it is employed to measure. The conservation theory is consequently not correct in the abstract sense. Our planetary system is altering its aggregate momentum, in keeping with the acceleration of its cosmic fall.

Descartes was right in diagnosing the gyrations of our system as vortical in principle, but he failed to substantiate his hypothesis because he conjured without the open-sesame of universal gravitation.

\section{The Precession of the Equinoxes}

In explaining the cause of the seasons, the books ordinarily speak of the earth's axis as remaining constantly parallel to itself, thus tilting the north pole toward the sun in summer and hiding it from him in the winter. This explanation suffices for beginners in the study of the science, but more advanced pupils are taught that the axis does not remain absolutely parallel, but revolves around like the axis of a common top when it is just getting ready to fall over. This movement in the earth's case is exceedingly slow, requiring almost two hundred and sixty centuries to complete it! The way astronomers originally discovered this fact was by noting the times of the recurrences of the vernal equinox. This has since been established to arrive about $20 \mathrm{~m} .23 \mathrm{~s}$. earlier each succeeding event, making what is known as our tropical (weather) year that much shorter than the sidereal one. 
But there is another way to measure this change, namely, by the length of arc described, and this amounts to 50.2" per annum. This circle of revolution can be and has been mapped upon the celestial sphere, or at least a considerable arc of it has been, from data which have come down to us through the past 3,000 or 4,000 years. Imagine the axis of the earth extended northwardly to the farthest sky, then the point of it, which now lies near the pole star, does not rest permanently in one spot, but travels anticlockwise around a central point, called by astronomers the pole of the ecliptic, but which is not marked by any star. The radius of that circle (mark the extraordinary coincidence) is $231 / 2^{\circ}$, which is just exactly the inclination of the earth's axis! What is even more remarkable is, that in all my reading. I have never found this curious fact alluded to, just as though coincidences such as this were quite the normal thing, or as though astronomers were all laboring under the erroneous notion that the causes which they assign for this phenomenon are likewise the causes of the inclination of the earth's axis.

Newton, groping in the dark, as I have already described, undertook to explain the fact, but not the coincidence. The phenomenon is known as the precession of the equinoxes. Newton's explanation may be thus stated: The equatorial radius of the earth exceeds the polar by about 13 miles, consequently there is a great ring of excess matter belting the planet and rotating obliquely with respect to the ecliptic. If this belt were in the form of a satellite, he reasoned, it would rotate around the earth agreeably to the same principles of rotation as the moon, and its orbit should therefore have nodes and exhibit a precessional movement of those nodes. But the ring, he went on, is not a satellite, but, on the contrary, is firmly affixed to the planet, consequently its natural nodal motion of rotation, being unable to express itself otherwise, must react upon the earth itself and cause a wobbling of that body's axis, technically called nutation. The tendency of the nodes to regress he found to arise from the fact, in the case of the moon, that 
that body is continually shifting from one side of the ecliptic to the other, and that the sun's attraction upon it is forever, though vainly, trying to bring it into the plane of the ecliptic, a condition that is no less true of the equatorial protuberance.

\section{Newton's Precession Theory Denied}

Now, the earth rotates on its axis once daily, thus bringing every inch of its equatorial bulge fairly under the sun's attractive influence. The cycle of causation is complete, why should not the cycle of effect be, also? Can you think of any sound reason why the earth's axis, if it is affected at all by this operation, should not make daily a complete circle of nutation, however minute, rather than the 1-365 part of the 1-26,000 part of a big circle? Again, the earth revolves around the sun in 365 days, presenting the equator in like manner to the sun's attractive influence at every angle of her axial inclination. Here the annual cycle of causation is complete, why should not its cycle of effect be annually complete also Why, indeed, should 26,000 complete cycles of causation, for no intelligible reason, save themselves up to make one big circle instead of 26,000 sucessive minute ones, and why, indeed, should the radius of that peculiarly generated big circle be so amazingly identical with the earth's axial inclination? What would you say of a man who, in cool deliberation, would protest that $9,490,000$ of our days and nights (26,000 of our summers and winters) should yield us a single day of daylight 13,000 years long and a single night of 13,000 years? Yet thus, in effect, is how Newton reasoned in this case.

Now as to the coincidence alluded to a moment since. Are the causes of precession assigned by Newton the causes also of the tilt of the earth's axis? Assuredly, they are not. For Newton's explanation of precession is, that because of the previously-existing obliquity of the equator, the causes of the former phenomenon were called into play. In short, his explanation of precession presupposes the inclination of the axis as an independent fact. 


\section{The True Cause of Precession}

Not only did Newton omit to take into account the outstanding factor of the stellar resultant, but he also violated his own third law of motion, namely, that every action has its equal and opposite reaction, by failing to deduce the fact of the sun's recoil. However, his delinquency in this respect was excusable and trifling in comparison with that of his army of followers who, despite their after-acquired knowledge of the sun's flight and of that remarkable instrument, the gyroscope, have lacked the acumen and initiative to divine that the sun's journey in space is not the ultimate, uncaused abnormality heretofore surmised, but the plain, dynamical effect of patent causes.

In order to present my own conception of the functioning of the Prime Resultant, of the cause of precession, of the nature and elements of the solar orbit, of the significance of the poles of the ecliptic and the geographical poles, etc., I shall request the reader to picture to his mind's eye an immense sphere suspended in space (compare Figs. 3 and 4) on the order of 4,000,000,000,000 of miles in diameter and to imagine inseribed thereon an equator and Arctic and Antarctic circles. We will call this the Gravisphere; and the center of it, to which the axis of the earth is continuously directed, will be the Vertex. Next, I will ask you to imagine the Antaretic zone of this sphere to be sliced off and removed, exposing to view the plane of that Circle. This plane, then, in my conception, is the invariable plane of the solar system and its boundary (Fig. 4), is the line of the sun's path, around which, as we look down upon it from the south, he revolves (carrying his system, of course, with him) in the contra-clockwise direction; he himself rotating on his axis, and the planets revolving around him, in just the opposite way (as indicated by the arrow points). The size of this orbit is indeed stupendous, measuring, as will later be shown, no less than $750,000,000,000$ miles from the Centrum (its center) to its circumference; that is, about 270 times Neptune's distance from the sun, or 8,000 
times our own distance. An observer, therefore, stationed at the Centrum would require a telescope of no less than 300 diameters to see the sun as more than a mere point of light.

It being out of the question to attempt to draw the figures to scale I have been compelled, in order to exhibit the earth's relations to the general scheme, to magnify the proportions, both of herself and of her orbit, countless thousands of diameters. Note, that in all the positions in which she is shown, her axis persistently points to the Vertex, the latter, as already explained, being a blank point in the line of the Prime Resultant. The consequence of this arrangement is, that in our circling of the sun's orbit with him, the Vertex is projected in the line of our sight against the celestial sphere, on which it consequently describes the so-called precessional circle, of radius $231 / 2^{\circ}$, around the pole of the ecliptic.

Turning our attention more particularly to Figure 4, it will be observed that our winter solstice occurs when the earth is farthest within, and our summer solstice when she is farthest without, the line of the solar orbit; also, that the vernal equinox occurs when the planet crosses the sun's path in front of him.

Thus at one point, say $\mathbf{A}$, the sun at vernal equinox would be projected upon the sky in a certain direction, while at B it would be projected one quadrant to the west. To an observer on the earth, therefore, viewing the sun at the time of the vernal equinox in successive years, the path of that body will seem to be toward $270^{\circ}$, right ascension. Again, the declination of the sun will likewise suffer a change in journeying the quadrant from $\mathrm{A}$ to $\mathrm{B}, \mathrm{a}$ change which can be resolved into two factors, namely; first, an uprighting of the axis to the extent of $231 / 2^{\circ}$ in one direction; and, second, a tilting of the axis to the same amount in a new direction at right angles to the former. The net effect upon the apparent movement of the sun in declination, then, will be, not the sum of $2312^{\circ}$ plus $231^{1}{ }^{\circ}$ but the hypothenuse of the right angled triangle two of whose sides are equal to $2312^{\circ}$ traced on the celes- 


\section{THE GRAVISPHERE}

Explanation:-This is an imaginary figure designed to portray the relation of our solar system to the universe at large. Observe that the south pole is placed at the top; this has been done purposely in order to impress the lesson that our cosmic fall is toward the north celestial pole. It has been found desirable, for the sake of avoiding confusion, to represent the Gravisphere as stationary and the orbit of the sun (along the Antarctic Circle) as closed; but the reader should conceive of the whole Sphere as falling the length of its own diameter $(3,836,000,000,000)$ in the period of some 20,630 years. The arrow-heads in the line of the Circle indicate the direction in which the sun is moving; but were it correctly represented, the path should actually appear as a deep spiral coil with a sheer fall equal to its horizontal circumference and a total length of $6,800,000,000,000$ miles. The Prime Resultant is shown in three different positions, but in reality it follows the sun's movement, describing the cone that has the Antarctic Circle for its base. The earth is drawn (vastly enlarged in proportion) in four different quadrantal positions, 6,452 years apart, in order to exhibit the nutation of its axis whereby the Circle of Precession is produced. 


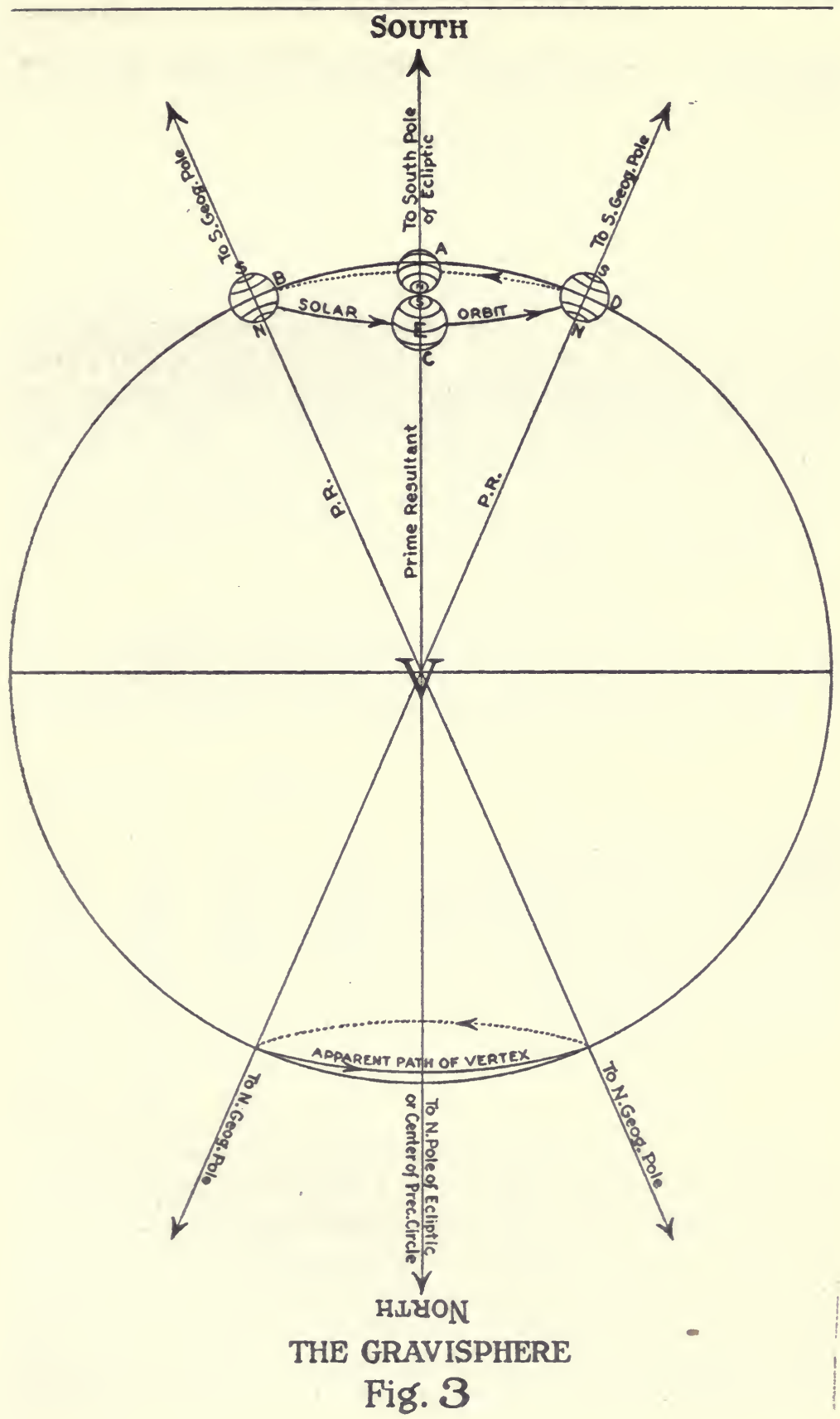


tial sphere. Applying the appropriate rule, then, we have:

$$
\sqrt{2 \times(231 / 2)^{2}}=33.23
$$

That is to say, the drift of the sun will seem to anyone not in possession of the key to be, not merely to right ascension $270^{\circ}$, but also to declination $+33^{\circ} 13^{\prime}$. On a similar line of reasoning, it can easily be seen that the vertices of preferential motion of the stars should appear to be respectively in R.A. $90^{\circ}$, Dec. $+11^{\circ} 45^{\prime}$, and R.A. $270^{\circ}$, Dec. $-11^{\circ} 45^{\prime}$, proving that the so-called star streams are nothing other than an optical illusion. Now compare these results with those derived by others by the laborious and conventional methods of empirical observation and analysis. I quote from Doctor W. W. Campbell's Stellar Moticns (p. 147):

Below are solutions for the apex of the sun's motion and for the vertices of the two star streams, by Kapteyn, Eddington, and Dyson; and for the positions of the solar apex and of the vertices of Schwarzschild's ellipsoid of preferential motion, by Schwarzschild, Beljawsky and Rudolph.

\section{Apex Vertex}

Kapteyn-Bradley stars

Eddington-Groombridge stars

R. A. Dec.

R. A. Dec.

Schwarzschild-Groombridge stars

Dyson-Stars of large proper motion

Beljawsky-Porters stars

Eddington-Zodiacal stars

Rudolph-Bradley stars

Rudolph-Bradley stars

\section{Means}

\begin{tabular}{llll}
$266^{\circ}+31^{\circ}$ & $951^{\circ}+13^{\circ}$ \\
266 & +33 & 93 & +6 \\
281 & +42 & 88 & +24 \\
281 & +36 & 86 & +24 \\
268 & +26 & 96 & +6 \\
& & 96 & +7 \\
& & 87 & +11 \\
\hline 272 & +34 & 93 & +12
\end{tabular}

Before we can label these conclusions final, however, we cannot neglect to take into account the optical effect of the sinking of the ecliptic on the apparent position of the stars. For it is obvious that, inasmuch as our descent is northward, those stars on or closest to this plane which are nearest us in point of distance will appear as drifting southward, whereas those more distant-and in the order of their distances-will seem to be traveling toward the 
north. Nor is this the whole story; for on keener scrutiny it will be discovered that the ecliptic is not sinking in continuous parallelism with itself, but is tilting at the rate of a small fraction of a second per annum, betokening in my opinion not a secular change, but a revolutional movement on the part of the Gravisphere, not, however, on its axis, but around a "centrum," vastly remote, indeed, yet not insusceptible of a rough approximation both as to location and distance.

A logarithmic spiral is defined as one that intersects all radiants at the same angle. This is precisely the ensemble arrangement we should expect of the planets in their orbits at successive instants of time, if, as I have been arguing, the planets are truly balancing themselves as a composite unit on the pivot of their lowest center of gravity. Strangely enough, astronomers have lately shown that spiral nebulæ (which for this purpose possess an advantage in having continuous arms to guide the eye, whereas a system like ours has only points) conform to this very shape.

It may be objected by some of my readers that the axis of the sun, Jupiter, and Uranus are not at all in alignment with the earth's axis. The objection, on the face of it, is sound and legitimate, and deserves a categorical answer. Let it be premised, however, that the only planet enough like our earth in constitution to serve for a criterion, to-wit, Mars, points its axis so nearly like the earth's that Doctor Lowell, late director of the Flagstaff Observatory, in a bulletin issued shortly before his death, asserted that its inclination is identically that of the earth's. Why this remarkable coincidence? Shall it be waved aside as immaterial? Moreover, Saturn's axial inclination is given by Flammarion as $25^{\circ} 42^{\prime}$ as against $23^{\circ} 27^{\prime}$ for the earth. Close enough surely to constitute a prima-facie case! As far as the definite exceptions of the sun and Jupiter are concerned, I might put astronomers in general on the defensive by saying, truthfully, that Newtonian theory doesn't account for the direction of even a single one of these axes, let alone for 


\section{THE SOLAR ORBIT}

Explanation:-On account of the immense disparity in the dimensions, it is impossible to draw this figure to scale. Its purpose is to illustrate the relation of the earth's axial inclination and her general movements with reference to the sun and his orbit. The large circle is, of course, the Antarctic Circle of our Gravisphere and represents the sun's path, or rather the path of the center of gravity of his system. The next smaller circle denotes the orbit of the earth, but relatively enormously magnified. Arrow-heads in all the circles indicate the direction of movement. The positions $A, A^{1}, A^{2}, A^{3}$ show the earth at the instant of her vernal equinox, $B, B^{1}, B^{2}, B^{3}$ at our summer solstice, and so on. The celestial point of vernal equinox is found by sighting through the center of the sun from the center of the earth at the positions $\mathrm{A}, \mathrm{A}^{1}, \mathrm{~A}^{2}, \mathrm{~A}^{3}$. Unfortunately, however, owing to the fact that the sun's center is less steady in its course than the center of gravity of his system, the data of observation are not as consistent as they otherwise would be. The letter $\mathrm{C}$ denotes the center (Centrum) of the solar orbit and $\mathrm{V}$ the Vertex, the latter, however, needing to be pictured, not in the plane of the paper but about five inches behind it. Here the orbit is shown as a closed curve, whereas in reality it is a spiral. To obtain the correct conception the book should be held horizontally and then slowly lowered away from the eye, at the same time imagining the rotatory movements as in progress. Note that our view is from a point south of the ecliptic. 


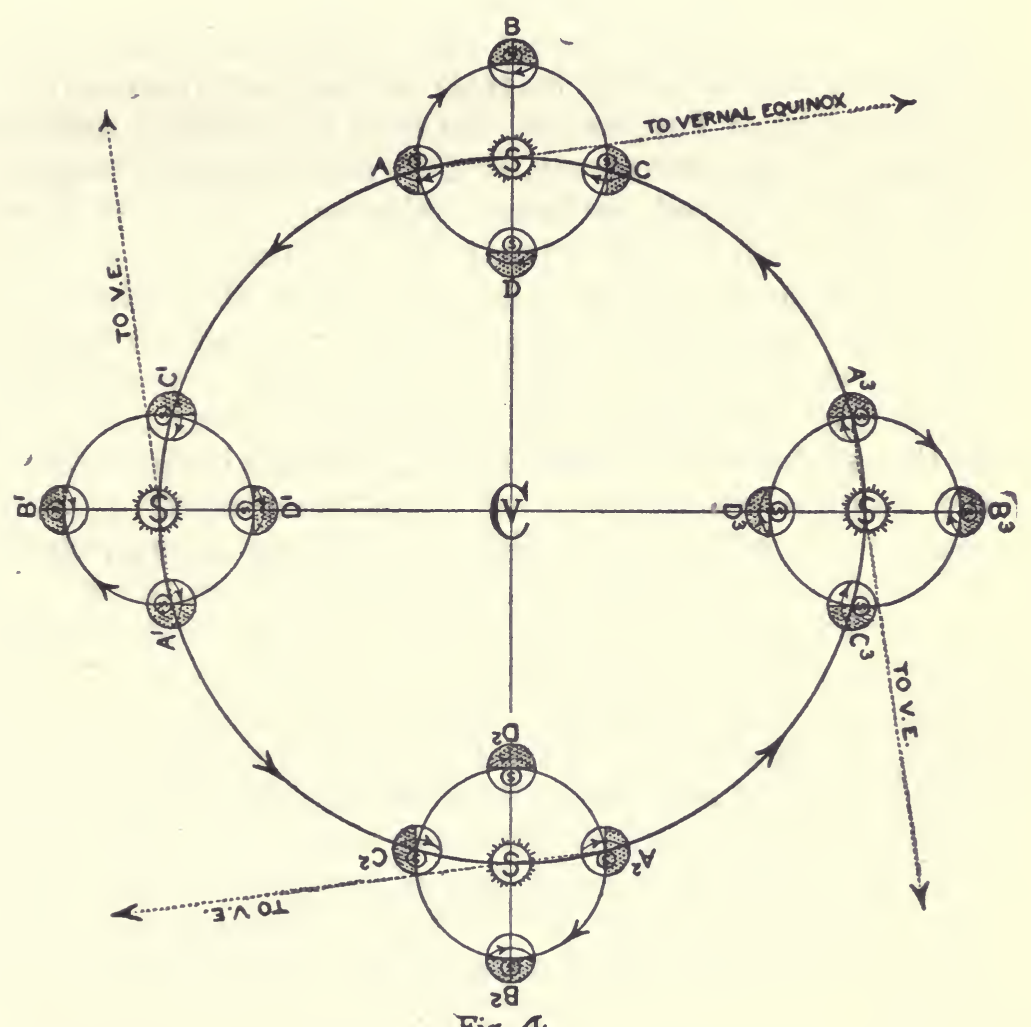

Fig. 4 
these three amazing coincidences. But I will not thus evade the question.

When you look at the earth, you see its crust; when you look at the sun or Jupiter, you see only their atmospheres, or, at least, fluid envelopes. You cannot, therefore, predicate with the same positiveness with respect to the hidden axial inclinations of these as you can with regard to the plainly visible ones of Mars and the earth. In the case of the earth, its interior (at least relatively to the interiors of Jupiter and the sun) is solidified, and incapable of free movement, so that her ballasting adjustments are confined, perhaps exclusively, to the surface, where they can be duly interpreted; whereas the sun, and, in all likelihood, Jupiter, also, are molten throughout, so that their ballasting process may go on inside, secretly, leaving their mobile surfaces freely open to centrifugal balancing. The force of this explanation is strengthened by the established fact that both these huge bodies exhibit the singular phenomenon of "equatorial acceleration"; that is, the rotation of neither is uniform from pole to pole, but is faster at the equator than in the latitudes.

You may ask me why the solar system rotates on its axis from west to east instead of just the reverse. I do not know, any more than I know why the water in the basin sometimes turns toward the right, and again toward the left. All I can say is, it had to move one way or the other, and then stick to that way. The die could have been cast by the first comet that crossed the nebular field, just as the mere touch of the tip of your finger on the water in the basin will determine the direction of its rotation.

It would seem to follow that, inasmuch as the rotations in our system are accelerating, the time periods are intrinsically shortening. On the other hand, however, the faster bodies are whirled the farther out they fly. For reasons which seem to me convincing, I believe that the earth is receding from the sun by infinitesimal degrees, and also that the year is shortening as well. This, however, is needlessly speculative and I will pursue the idea 
no further. The thing of importance is, that we have ferreted out the hidden force that whirls the planets around and counteracts the centripetal attraction. 


\section{V}

\section{THE LAW OF EQUILIBRIUM}

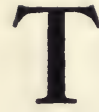

HAT objects near the earth's surface seek their lowest center of gravity, or, what is the same thing, their equilibrium, was known long before Newton's time quite as well as it is in ours. Indeed, it may truly be said to have been better understood then than now, for the reason that Newton, in his quest for the solution of the mystery of the tides, introduced a perversion of the law that has ever since obsessed the scientific mind and incidentally worked untold injury to the cause of astronomical science. This pseudo doctrine of which I speak teaches that bodies lose the power of self-balancing when falling in vacuo, and is founded upon what is commonly known as the vacuum-tube experiment, which is thus succinctly described in Ganot's Physics (Art. 77):

Since a body falls to the ground in consequence of the earth's attraction on each of its molecules, it follows that, everything else being the same, all bodies, great and small, light and heavy, ought to fall with equal rapidity, and a lump of sand without cohesion should, during its fall, retain its original form as perfectly as if it were compact stone. The fact that a stone falls more rapidly than a feather is due solely to the unequal resistance opposed by the air to the descent of these bodies; in a vacuum all bodies fall with equal rapidity. To demonstrate this by experiment a glass tube about two yards long may be taken, having one of its ends completely closed, and a brass cock fixed to the other. After having introduced bodies of different weights and densities (pieces of lead, paper, feather, etc.) into the tube, the air is withdrawn from it by an air pump, and the cock closed. If the tube be now suddenly reversed, all the bodies will fall equally quickly. 
On introducing a little air and again inverting the tube, the lighter bodies become slightly retarded, and this retardation increases with the quantity of air introduced.

Now, it is a sound and cardinal rule of experimental science that the experimenter shall not allow his personal predilections to color the result. Unfortunately, Newton and, worse still, all who have since been led to repeat this particular experiment have started out with no other possibility in mind than that the moon is the major cause of the tides; and it is therefore not at all surprising that all of them alike have fallen blind victims to the same blunder. Nor, when we recall the countless instances in the past of the tenacity of traditional error, should it be any more surprising that Newton's tidal hypothesis has survived in human opinion the positive disproof of the same by Nature herself.

The worst fault I have to find with modern astronomy is its impotency to rid itself of the ancient propensity of trying to interpret the phenomena of the firmament by systematically differentiating between terrestrial natural law and celestial natural law. The one great truth I seek to inculcate is that natural law is everywhere identically the same, from center to circumference of the physical universe, be it finite or infinite. Metaphysics and physics are independent worlds. You cannot rationally postulate uncaused rectilinear physical motions or assert that persistence and inertia are equivalent terms and still claim to be a scientist in the ideal sense. Here in this question as to the universality of the law of equilibrium we have another such case in point. It would seem that Newtonians look upon the principle of equilibrium as a sort of incubus upon matter, a mistake of Nature's as it were, and that they feel that they are performing a signal service to her by cogitating this base mundane impediment out of existence in the "nobler" universe above us. Terrestrial objects, say they, are bound by the law of equilibrium; celestial bodies, on the contrary, are of a higher order and are absolved from such servile obedience. With them "celestial me- 
chanics" is not terrestrial mechanics applied to stellar objects, but rather an elaborate transmogrification of it. Where Newton did not hesitate to invoke divine agency to explain such physical wonders of the heavens as he himself could not fathom, his followers, more materialistically given, have aimed to supply the place of the Deity by the banality of alleging such wonders to be "natural", without taking over the burden of showing why and how they are natural. They convince themselves that the sun and the stars are dashing through space, and then they go on and say it is "natural" for all celestial bodies thus to dash through space, and that the phenomenon "demands explanation only as mere existence does." They behold one member of a binary star circling about its fellow, so they declare "the central one whirls the other round and round," and that such behavior is natural, up in the sky. They see the earth rotating on its axis, and this again they sagely inform us is natural, also, else why, forsooth, should it be so rotating? Furthermore, seeing the equatorial ring, they say that such momenta as inhabit planetary bodies are different "by nature" from terrestrial momenta in this, that they are "persistent," and can go on doing work forever without any fresh feeding of the motive power. All these so-called laws (!) they affect to believe true of celestial mechanics; and, in line with this policy, they teach that the vulgar law of equilibrium is altogether too corporeal to consort with these supernal archetypes.

Far from being a clog upon matter, whether here or in the sky, whether in this paper weight or in the terrestrial globe of which it is a part, the principle of equilibrium is the indispensable attribute that ensures the preservation and regulation of the universe in all its activities and through all its broad extent. Here on earth, is not equilibrium the ruling factor that makes for order as against confusion, for system as opposed to chaos? Why should it be preferentially assumed, then, that, in the greater cosmos, the principle should prove itself otherwise? As well might one advocate the abolition of 
rudders as being an impediment to ships, which otherwise would be free to move indiscriminately. The one great truth, rising mountain high above all others in the realm of physical science, is, that natural law is irrevocably unchangeable, and that, for all its inflexible rigidity, the universe it controls is at once fraught with kaleidoscopic changes of infinite range, yet withal perennially maintained in order. So must it ever continue, as ever it has continued.

\section{Newton's Vacuum-Tube Experiment}

In the first place, let it be remembered that this experiment was designed and undertaken with but one object in view, namely, to lend color to Newton's rashly-conceived hypothesis regarding the cause of the tides. Had subsequent investigations of the tidal phenomena borne out the hypothesis, the fact would have constituted an all but conclusive confirmation of the basic lesson drawn from the experiment. Far from this being the case, however, we are informed by such lofty authorities as Darwin, Kelvin and Young-Newtonians all par excellencethat the tidal hypothesis is "utterly unsatisfactory in regard to what actually takes place," and that "it is nearly as much wrong as possible." If there is any merit in the logic of facts, then, it follows that, the hypothesis having been weighed in the balance of Nature and found wanting, every link in the chain of deduction is forcibly brought under suspicion. The staple link in this chain is the vacuum-tube experiment; why, then, should its soundness be taken for granted? In view of the disastrous outcome, is not the probability precisely the reverse?

In the next place let me ask, What is the physical or causal relationship, if any, subsisting between the surrounding medium and the equilibristic tendency of any object therein contained? Is there, perhaps, some secret virtue in the atmosphere that, passing into the object, imparts to it the power or imposes upon it the obligation to lower its center of gravity? And, conversely, does the withdrawal of the air from the receiver deprive it, pro 
tanto, of that power or absolve it from that obligation? If this is what Newtonians really mean to imply-and it is difficult to construe their attitude otherwise-they throw themselves open to a gross inconsistency, namely, in denying to the earth, in their tidal theory, the power of equilibrium, for the earth is blessed with a stupendous atmosphere. Moreover, supposing it true that the medium does possess such virtue, then unsymmetrical objects resting on the floor of the vacuum should be as exempt from the law as at any height within that chamber; whereas the undeniable fact is that such bodies upright themselves just as certainly as they do in air or water. It is as clear as day that in this case the medium functions only by moderating the velocity of the fall, thereby affording the investigator time and facility for studious observation.

Obviously, then, the principle of equilibrium exists altogether independently of the atmosphere. Its real essence-how can any one doubt it?-lies in gravitation, nothing else; and, like the latter, or rather because both are of the same essence, it can no more abandon any particle of matter, or be filched from it, than can gravitation itself. In olden days it used to be argued that when a balloon rose in air, it was because gravity had ceased to act upon it. One of the best answers to that fallacy was that the balloon, even when in the act of rising, continuously observes the law of equilibrium, keeping its heavier part underneath. To-day we have the same old dispute resurrected, but at the other end of the chain. Now it is the principle of equilibrium that is at issue. By means of an opposite sort of device, man has invented a way of causing objects to fall with even greater velocity than they do in air, and to fall so rapidly, indeed, that their subtle efforts at self-balancing successfully elude the keenest eye. Thus is the presence of the principle of equilibrium obscured now, as was the presence of gravity formerly in the phenomenon of the balloon. It is a poor rule, they say, that doesn't work both ways; Why not then apply the converse of the old reasoning? Why not 
say, inasmuch as the action of gravity on the balloon, in spite of its act of rising "against gravity," was legitimately inferable from the circumstance of the balloon's preserving its lowest center, so the action of the law of equilibrium, in the case of the object in the tube, though masked from our perception behind its high velocity, is reciporocally inferable from the bare fact of the fall?

How, do you suppose, was the existence of a center of gravity in objects originally discovered? By casually noting the fall of apples from trees? Certainly not! The unmistakable way was to choose a suitable object, immerse it in a liquid, and then watch its behavior. To this process two things were essential; the first being that the object examined be left perfetly free to turn upon itself ; the second, that it be kept steady enough to allow the eye time and opportunity for critical inspection.

Now, when Newton came to prove up his tidal theory, he found the knowledge of the law of equilibrium there before him, and he did not find it to his liking; for, as I have previously stated, it did not accord with his major premiss that the moon is the chief tidal force. Most of us have the faults of our virtues, and Newton was no exception. Always fertile in expedients, he was sometimes as inventive in the pursuit of error as he was at other times in the cause of truth. Precisely retracing the course by which the law had originally been brought to light, he one by one systematically restored all the obstacles that aforetime had blocked the road to its unearthing, and, by this unworthy subterfuge, undid discovery and relegated this inestimable diadem of knowledge back again into the limbo of the Unknown. His tube having to be of glass, to ensure transparency, and glass being by nature very fragile, it was manifestly impracticable to provide one of even moderate length, so it was actually made but five feet long. Moreover, the tube needing to be hermetically sealed as a necessary condition to the pumping out of the enclosed air, it became automatically impossible to introduce instruments for precise measurements, supposing such exactness to have been desired. Finally came the 
removal of the resistance by conscientiously exhausting the air. Having carefully gone through all these maneuvers and satisfied himself, for the last time, that Nature was now effectually gagged, he called to her: "Prove to me now in the wink of an eye that these objects, as they fall through this tube, obey your boasted law of equilibrium. If you fail, I will brand you for all time to come as the most inconstant of your sex." Newton made no allowance, nor wished to make any, for the brevity of the time, the extreme shortness of the fall, the persistence of retinal impressions, the notorious uncertainty of the human eye in general, his own mental biasall he aimed at was to confirm his already fixed opinion and to clinch his ill-fated tidal hypothesis. But Nature, after generations of misrepresentation passed, finds voice at last through Kelvin, Darwin, Young and the rest and proclaims that the hypothesis is "as false as can be." Alas, she has been silent too long, and not even her mouthpieces heed her testimony!

Ask any modern physicist whether a shell fired from a mortar observes the law of lowest center, and he will unhesitatingly answer, yes. He cannot see even the path of the shell, much less the gyrations of the missile upon itself, but for all that he is none the less positive. Why? Because he intuitively reasons to the truth beyond this negative evidence. He will sensibly argue, "I cannot perceive grass grow, but I know it does grow. I cannot detect the motion of the stars, and for ages mankind believed them absolutely fixed, but science has now demonstrated that they do move, and at astounding velocities. With my naked eye I cannot see animalcules, but the microscope reveals to me their existence". All this he will say, and more; yet, in the end, he will reiterate with unabashed finality, "I cannot descry any sign on the part of the object falling in the vacuum to seek its lowest center of gravity, ergo, I deny that it does so."

If there is anything that science can take just pride in, it is in those inventions which have extended human knowledge into domains beyond our direct sense percep- 
tions. The telescope revealed to Galileo the rings of Saturn, the four moons of Jupiter, and the phases of Venus; and improvements of the instrument have brought to light countless other wonders. No doubt the bigoted Libri, Galileo's bitterest critic, had he had the power, would have been glad to reverse the telescope for all time, as a means of guarding mankind from the perils of unbelief. In effect, that is just about what Newton did. He found humanity possessed of the knowledge that bodies seek their lowest centers of gravity, a knowledge which had previously been arrived at by painstakingly bringing within the purview of human perception what before had been hidden from sight; and, for mere expediency's sake, he deliberately suppressed that knowledge by snuffing out the torch by whose light it had been revealed. In short, he reversed the glass. For what use is it that man has invented the telescope, the microscope, the thermometer, the barometer, the bolometer, the spectroscope, the photographic plate, etc., if their valuable evidence is thus to be arbitrarily set aside to suit the exigencies of crazy hypothesis?

Of course, there is the possibility that many scientists are already convinced, not necessarily from any arguments I have here advanced, but as a result of their own private reflections. However, they have none of them, so far, seen fit to speak out; possibly because of a wholesome and well-founded dread of the hierarchical lash. Your Newtonian professor may, indeed, say with Darwin that the tidal theory is as false as can be, but he can always be depended upon to subserviently couple the heresy with the retraction that he believes in it notwithstanding, and that he will continue faithfully to teach it to his classes as one of the gospels of science. You may, however, test his sincerity, if you will, after some such manner as the following:

Provide yourself with a pair of false dice so heavily loaded as to turn up sixes at every throw, and let the casting of pairs of sixes constitute the winner. Exhibit these to any Newtonian of your acquaintance, not concealing 
from him their fraudulent character. Then, taking a perfectly true pair of dice from your pocket, hand them to him and say: "Here, friend, is a pair of honest dice for you. Let us throw for the dinners. Do you use your dice, casting them in the ordinary manner in the open air, but let me shake my false ones within a vacuum tubepairs of sixes to win." If he is consistent at heartscruples of conscience aside-he should take you up on the instant. But will he? Think the matter over.

Theoretically, it would be possible to extend the original vacuum-tube experiment to the other extreme; that is, we may suppose the tube lengthened to, say, ten miles. In that case the act of falling would consume just about one minute-surely not too long to grant Nature a fair chance to absolve herself from the stigma of vacillation that Newtonians have thrust upon her. Incidentally, we may imagine the tube capacious enough to allow for the introduction of suitable micrometrical devices to record the equilibristic gyrations of the object, not only at the instant of alighting, but also in the course of descent. Of course, all this is practically impossible, and if Nature must await her vindication until such a thing becomes practicable, she is likely to remain in disgrace until all human interest in the issue has died out. Fortunately for her and us, however, she has thought to set out documentary evidence of her consistency on the scroll of the sky. I refer to the moon. Why, do you suppose, does our satellite continually turn the same face toward us, unless it is because her visible hemisphere is the heavier; and why does she exhibit her librations, if not because of the conflicting attractions of the sun and of the Prime Resultant?

Now conceding-solely for the sake of argument, however,- - that the moon is really falling earthward at the rate of 1/19 inch per second, then the earth, being 81 times heavier, must be falling moonward only 1/171 inch in the same brief instant. This is tantamount to saying that the earth, if falling moonward at all, is falling only one inch in about 3 minutes! Suppose that by some de- 
vice, as by manipulating a switch, you could control gravitationally the velocity of a loaded die in the tube so that it would fall as slowly as this, thereby giving you ample opportunity to observe it closely, and it abundant time to adjust itself,-Do you conscientiously doubt that the die would turn its loaded side underneath, just the same as it would if sinking slowly in a glass of water?

Here I fancy the reader exclaiming, "But you are mistaken in asserting that Newton predicated his conclusion upon the behavior of the individual object; he, on the contrary, inferred it, and correctly, from the fact that objects of very diverse densities, such as a feather, cork, bullet and the like, fall with exactly equal velocity."

My first reply to this is, that when you use the word "exactly" in this connection, you display a confidence in your seeing skill that I am far from feeling in my own. For my part, I think that there is as much, and more, uncertainty in correctly deciding the race between two particles, such as a long feather and a tiny spherical bullet, traveling but a yard or two of height at great velocity, as there is in deciding, for example, close plays at second base in a professional game of baseball. Umpires, at least, know how little dependence is to be placed on the visual judgment of the average "fan." It is truly amazing the concordance of opinion between the umpire and the crowd when his decision favors the home team, and equally astounding how little that same umpire's judgment is respected when he renders his verdict in favor of the visitors. In the case of the particles, everybody, from Newton down, has been all along betting on the race as a tie, with no takers, and it is not to be wondered at that the verdict is enthusiastically unanimous.

But even admitting the cogent argument of "preponderant opinion," it is quite as much of a non sequitur to hold, that because objects fall in vacuo with identical velocities, they individually repudiate the law of equilibrium, as it is to assert, that a pellet of lead and a pellet of chalk of exactly the same size and shape, likewise falling together in a vacuum tube, will strike the bottom with 
equal force because they are traveling with equal velocities. Two racers may easily cover the specified distance in unison, but that doesn't preclude each from balancing himself separately, or regulating the length of his own stride.

But even waiving all that has gone before and taking the Newtonian experiment at its face value, I still take exception to it, not merely because it does not reproduce the cosmic conditions, which may be excusable, but because it does not sensibly parallel them. The Newtonian deductions, in the light of the experiment, may be thus itemized :

1. Objects in vacuo, irrespective of their varying densities, fall with precisely equal velocities.

2. Cosmic bodies are only objects of a larger growth, are constructively falling, and are falling through the void of space; therefore they, also, and all their component molecules fall with equal velocities.

3. All objects, great and small, thus falling in vacuo maintain throughout their descent both their initial inclinations and their original shapes.

Let us try this conception out by analyzing a simple hypothetical case:

Imagine, if you please, that some power should arrest the moon in her orbital flight, hold her steady for a moment, and then gently drop her. According to Newtonians, she would fall in one attitude straight downward to the earth, where she would arrive in something less than a week's time. Suppose, again, that the same power that arrested the moon should at the same moment cause to spring into existence, round a point just 3,000 miles this side her center, a globe of water of exactly the same mass, and that both these moons were allowed to drop simultaneously. What would be the result? Were we to apply the reasoning of Newton, as based on his aforementioned experiment, we should have to argue somewhat after this fashion: Here are two great "objects" falling together through a vacuum. They must 
therefore fall with precisely the same velocity, preserve throughout their respective initial inclinations and shapes, retain their separate individualities, and, finally, strike the earth one after the other. Or, as Ganot puts it, "Everything else being the same, all bodies, great and small, light and heavy, ought to fall with equal rapidity, and a lump of sand without cohesion should, during its fall, retain its original form as perfectly as if it were compact stone."

Such, I say, is the solution tendered us by Newton and his disciples. Let me now submit my own: Although it is quite true that each of the two moons is only 1/81 as massive as our planet, they are, on the other hand, eighty times nearer each other than their common center of gravity is to the earth. Let it be remembered, however, that attraction varies directly as the mass, but inversely as the square of the separating distance, whence it plainly follows that the net attraction between the moons is eighty times greater than that between their joint mass and the earth. This condition would result in a singular thing, namely: The aqueous ball, being solicited moonward far more powerfully than earthward, it would, for a time, actually rise away from the earth until it should meet the true moon on her way down. The impact of such a meeting would, of course, per se, deform both colliding bodies. But this would not be the sole result. The integral power of attraction of the coalescing mass would immediately come into play-a constructive, as the collision was a destructive force-and this would automatically remould the whole into a globular form, in which new state the merged moons, continuing their descent, would strike the planet as one.

Which of these analyses does the reader prefer, the first based on the denial of the law of equilibrium, or the second, founded on the law itself?

\section{KePLer's Laws}

Newton demonstrated to the satisfaction of expert mathematicians (who, by the way, alone can follow and 
comprehend his treatise), that Kepler's laws and the law of gravitation are in harmony. As the proponent of the proposition that the law of equilibrium is just as deeply rooted in this great law of gravitation as are Kepler's laws, it would seem to devolve upon me to establish, mathematically, the essential identity of all three. I shall therefore make the attempt.

The reader will remember that I recognize three sorts of gravitational units, namely: (1) a simple body, like the moon; (2) a compound body, like our binary earth-moon system, and (3) a complex body consisting of a multitude of planets and satellites, all balancing themselves around their common center of gravity. Moreover, I define a gravitational unit as a single body, or a congeries of cosmic bodies, seeking its lowest center of gravity. The proposition I am now going to try to prove is, that our solar system is a family of such bodies so intimately associated with each other, by virtue of their mutual attractions, and so distantly removed from the stars in general, as to behave as a consolidated mass in this: that, while in the act of falling in the direction of the resultant of the stellar attractions, they seek their common systemal center of gravity and revolve around each other according to the

\section{Law of the Lever or Balance Arm}

It is a fact already well recognized by astronomers, that the moon does not revolve around the center of the earth, but around the center of gravity of their joint mass, and that a similar principle holds good of the planets with respect to the sun. So far, then, the principle of the balance arm has been scientifically accepted. But this knowledge does not dispose of the riddle as to why these bodies rotate at all; it does not explain the impulsion that lay, or lies, behind those tangential or centrifugal motions, nor does it point out what keeps them going. My conception is, that the orbital movements of the circulating bodies is due to their act of falling at the 
command of the Prime Resultant, and that instead of falling down in straight lines, as they would do were their mutual attractions dissolved, they fall with a spiral twist that carries them perpetually round and round their common center of gravity. In short, the solar system, I hold, is an immense clock driven by its own descending weight.

This mechanical principle is capable of unlimited extension, upward and downward. It applies as well to molecules as to stars, to cohering particles as well as to cosmic orbs separated by the full span of the universe. The sun's next door neighbor is Alpha Centauri, ten thousand times farther from him than Neptune, his outermost planet. Think of this, or any, pair of stars, or of any combination of pairs or clusters of stars, in their relation to the sum of the universe, and there will be borne in upon you the realization that the whole body of the macrocosm is perpetually writhing within itself in the throes of equilibristic evolution. The physical universe is built on the principle of action, not stagnation; on that of automatism, not blind chance; on perpetuity, not finiteness.

Concentrating our attention on the solar system, we note that the planets revolve around the sun in (seeming) ellipses, that their radii vectores sweep over equal areas in equal times, and that the cubes of their distances are proportional to the squares of their periodic times. The problem before us is to prove that these phenomena are incidental to the normal operation of the principle of the lever-but, mark you, not of a stationary lever, but of one whose pendent weights are in the act of falling.

To begin with, what is the principle of the simple lever or balance arm? It is this: Suppose a bar to be supported on a pivot so as to rotate in a horizontal plane, then, in order that its arms, if unequal in length, shall balance, the weights at the ends must be inversely proportional to those lengths. That is to say, if one of the arms be half the length of the other, the weight on the shorter end must be doubled, if one-third the length, trebled, and so on. 
And what are the laws of falling bodies? These are given by Ganot (Art. 49) in these words:

1. The velocities are proportional to the times during which the motion has lasted.

2. The spaces described are proportional to the squares of the times employed in their description.

3. The spaces described are proportional to the squares of the velocities acquired during their description.

4. The spaces described in equal successive periods of time increase by a constant quantity.

Suppose a bar, whose longer arm we shall call $R$ and its shorter $r$, be rotated horizontally around a stationary pivot, it will then descibe two circles, and, by geometry, we get the equation,

$$
r: R:: 2 \pi r: 2 \pi R
$$

By our hypothesis, however, the pivot is not stationary, but is falling, and the ends of the bar are not describing closed circles in fact, but coils of open spirals. In still other words, the last two terms of our equation are, properly construed, "spaces fallen through." Under the second rule given above, then, our last two terms, in order to express the element of time instead of space, must be amended to read

$$
\sqrt{2 \pi r}: \sqrt{2 \pi R}
$$

At this point, let us not forget that we are not dealing with a simple bar, but with a lever, whose arms, in order that they may balance, must be weighted inversely as their length; that is to say, a weight $R$ must be attached to the end of arm $r$, and a weight $r$ to the end of arm $R$. Pound for pound, then, the time required to complete the running of the smaller coil as compared with that required for the larger is:

$$
\frac{\sqrt{2 \pi r}}{R}: \frac{\sqrt{2 \pi R}}{r}
$$

(3) whence, (parts of circles being to each other as their like parts)

$$
r \vee \bar{r}: R \vee \bar{R} \quad \text { (4) meaning; }
$$


The periodic times of planetary bodies are to each other as their respective orbital radii (distances) into the square roots of those radii.

Notice that this gives the ratio of the simple periodic times, not the ratio of their squares, hence is logically better; but if you wish to identify the ratio with Kepler's law, all you need do is to square the separate terms, thus:

$$
\begin{aligned}
r^{2} r & : R^{2} R \text { or } \\
r^{3}: R^{3} & \text { q.e.d. }
\end{aligned}
$$

Again, since under the third law given above, veloci ties are proportional to the square roots of the spaces (here circumferences, or orbits), they are necessarily proportional in like manner with respect to the radii, or as

$$
\vee \bar{r}: \sqrt{R}
$$

But we are not to forget that these velocities are directly proportional to the number of units of weight that run the course, or, in other words, they are inversely proportional to the radii, or lever arms, whence:

$$
R \vee \bar{r}: r \sqrt{R}
$$

(8) meaning,

The velocities of planets are proportional to their respective distances into the square roots of their oPPOSITES.

With this formula (8) before us, it is easy to derive the law of gravitation, gravitation being a form of ENERGY. Energy being proportional to the square of the velocities, we have, then,

$$
R^{2} r: r^{2} R
$$

Now, there is this further rule regarding energy of motion, namely, that it varies directly as the load that has been successfully carried; and, as we have seen, the heavier load on a balanced lever is at the end of the shorter arm. We therefore multiply the first term by $\mathbf{R}$ and the second by $r$, obtaining:

$$
\begin{aligned}
& R^{3} r: r^{3} R, \text { or, } \\
& R^{2}: r^{2} \quad \text { q.e.d. }
\end{aligned}
$$


Which is the law of gravitation itself, namely, that the energy of planets' motions varies inversely as the squares of the radii (distances). This method really gives the centrifugal force, but as the centripetal is, by the law of reaction, its equal, the expression given is true for both.

\section{The Law of Areas}

Kepler's second law declares that the radius vector sweeps over equal areas in equal times. This can be proven, on the principle of the falling lever, in this way:

Suppose a planet to describe a certain are at perihelion in the space of one hour and, later on, an are at any other part of its orbit, say aphelion, in a like space of time. Regarding the arcs thus described as ares of circles, and the differing distances as radii, $r$ and $R$, the arcs, geometrically, will be in the ratio of

$$
r: R
$$

We are not dealing with plain circles, however, but with the rotations of a lever. According to this principle, what is gained in power is lost in velocity, and vice versa. Moving the planet out to aphelion, therefore, modifies the equilibristic lengths of the arcs described in the inverse ratio of the radii, hence the arcs become,

$$
R r: R r
$$

But the ares thus related are not plain distances, but spaces, through which the weights (which in the present instance are equal, being, indeed, the same planet) are falling; hence we now find the opposite ares to be proportional, dynamically, thus

$$
\sqrt{R r}: \sqrt{R r}
$$

But, by geometry, the areas of circles are as the squares of their circumferences or of their like arcs. In our ratio we have what we may correctly describe as dynamical arcs, incorporating within them, as they here should, the ideas of geometrical relationship, equilibrium, and accelerative motion. Squaring the terms, then, we get,

$$
R r: R r \quad \text { q.e.d. (15), meaning, }
$$


that the areas of the respective segments at perihelion and at aphelion covered in equal times are equal. Of course the rule holds good whatever parts of the orbit be compared.

Kepler's first law, namely, that planets travel in ellipses, is true only in the most superficial of senses. It is not true geometrically, dynamically, philosophically, or actually. Planets travel, instead, in open elliptical spirals of an exceedingly complicated design. Astronomers, indeed, acknowledge that the orbits are not reentering, inasmuch as the translation of the sun is well established; but they assume that, even if the sun were stationary, the planets would, nevertheless, continue revolving, and that these orbits would then veritably be closed curves. They picture the sun as possessing a unique rectilinear motion not primevally shared by his planets, and that the reason they accompany him is because he is dragging them along as a horse does a vehicle, whether or no. Thus have the Newtonians, in a way become habitual with them, underestimated the most significant and potential facts of the cosmos, facts, too, gained by their own herculean labor and expense.

Even though the bores, or calibers, of planetary spirals admittedly exhibit elliptical curvatures, the fact conveys little hint of the dynamical agencies concerned in their generation. Profoundly interpreted, each infinitesimal arc in each and every one of the planetary- and satellite spirals is a coördinated resultant of the equilibristic adjustments of the aggregate system; that is to say, every movement and turn of movement of the planets is a concomitant. effect of each member balancing itself against all the rest. The solar system, in fine, is essentially a revolving, composite lever. To preserve the common equilibrium, when one member moves out from the sun another or others must, perforce, move in, and vice versa. These compensations automatically follow from the principle of balance, and extend to every part, even to the molecules and atoms that hang suspended in space; even, indeed, to the ether itself, if it be amenable to grav- 
ity. These compensating pulsations of the planets in and out from center have not escaped the keen eyes of astronomers, and have led to the empiricism and near-truth that the moment of momentum of the system preserves its uniformity. Unknowing the dynamical cause behind the phenomenon, they construe it as a teleological ordination, and point to it as the unimpeachable evidence of the socalled doctrine of the conservation of energy.

The fact that the planets have accommodated themselves all to practically one plane (that is, to a position of "flatness"), which, as I have shown before, is the condition of maximum stability, joined to that other significant fact that their orbits are near-circles, demonstrates either; first, that the planets are direct offsprings of the sun, or, second, that they have been members of the system so inconceivably long as to be in effect indigenous to it. It is otherwise with the comets and many of the asteroids, whose eccentricities of elongated orbits, high inclinations and retrograde motions are so many unmistakable proofs of their alien parentage and comparatively recent immigration. Like the bubble of a spirit level when first applied in test, a comet takes a series of pulsations across the field of equilibrism before attaining the state of relative rest that belongs to perfect balance.

\section{The Path of the Sun.}

Sir William Herschel (1738-1822), the illustrious discoverer of the planet Uranus, was the first to indicate the proximate point in the heavens toward which the sun is tending. That point astronomers refer to as the apex of the sun's way and now (erroneously) declare it to be "in the direction of the constellation Hercules, about $10^{\circ}$ southwest of the star Vega" - not many degrees, indeed, from where Herschel himself located it. Since Herschel's day, numerous astronomers have adopted this particular field of research as their life-work, and hundreds of thousands of dollars have been expended in one form and another in this quest. Every labor of great magnitude 
such as this is should have behind it an adequate motive. Has this inquiry such a motive?

It is a lamentable thing to have to say, but the truth should out, that from the first day to the last that this search has lasted, scientists have had no motive other than merely to ascertain the bare isolated fact, entertaining neither plan, expectation, or hope that the knowledge will, or by any possibility ever can, prove structurally useful. Herschel has been dead for nearly a century, yet in all these years, for all the efforts made to reduce to exactness what he only adumbrated, there does not appear to have been a single illuminating suggestion put forward as to how the knowledge might one day be made helpful in the upbuilding of the science, be that knowledge as exact as ever it may.

Newtonians rely on the fundamental sophism that the motions of cosmic bodies are self-existent; that they are no more susceptible of explanation than matter's existence. Of our sun they say, simply, that he moves. They do not ask why he moves, for that riddle they gave up from the start, supinely supposing it beyond the reach of human penetration. All they dare to ask, or tolerate cthers asking is, "How fast and in what direction is he moving?" for this question involves no radical innovation which might in the end spell disaster to their cherished prejudices. It seems self-evident enough to me that the Newtonians, in denying the causation of the sun's motion, and in asserting, with ever increasing assurance and solemnity, that his course through the ether is unique, random, and uncontrolled by any organic unifying law, are guilty of a grave folly and are needlessly renouncing in advance the choice fruits of their arduous labors. The only conceivable value the knowledge of the sun's course and velocity can have to science and humanity, lies in its possible far-reaching correlations, in its constructive potentialities. A sun moving, as they assert, randomly, unimpelled, and undirected, can have, per se, no correlations and no structural significance whatsoever. 
For all that, the practical astronomers, fired by the passion for physical accuracy for its own sake, are every day planning on an ever more and more ambitious scale to go over the same ground again and again, without any definite object in mind, it seems, save to see how near they may come to the previous marks. There is no way to test their accuracy, so far as they are aware, by final arbitrament of Nature, but only by indecisive comparison of their own results with those previously announced. In any case, all their labor goes for naught as long as their architects, the theorists, neglect and refuse to reform their ground plans so as to give structural place to the plethora of facts (never anticipated by the original architect, Newton) now promiscuously encumbering the ground. Whatever incentives one may have to remaining unpersuaded, it must be patent to all that $a$ radical reformation of astronomical theory has become imperative. What more likely way, I ask, than by giving to the phrase "UNIVERSAL gravitation" its plenary significance and genuinely universal applications?

The principle of the gyroscope is too well recognized to require extended discussion in this place. Rotation in one direction around an axis, under the law of action and reaction, must, and as a matter of everyday mechanics does, generate a reverse movement of the rotating mass in a larger circle around an extraneous point or axis. We are not merely angling at random, then, when we posit that the solar system, because of its integral rotation around an axis within the sun's mass, has a secondary motion of precession around just such an extraneous axis. The existence of such a reaction is a physical necessity, not a figment or invention, and any cosmological system that omits it is ipso facto valueless. The sun moves - must move-in an orbit. It needs no demonstration; for the law of action and reaction and the principle of the gyroscope leave no other possibility open.

It is by no means a novel suggestion that the sun may be pursuing a curved path. Ever since the time of the elder Herschel, and probably long before him, the 
idea has been a subject of speculation. But to the eye of Newtonian philosophy the sine qua non is, the presence of a central force. From time to time they have considered the eligibility of such great stars as Sirius, Canopus, Arcturus, Alcyone, Orion, and even certain star clusters, as hitching posts to which to tether the universe; never once suspecting the cosmic virtues inherent in the stellar resultant. At the present time the consensus of astronomical opinion appears to echo that of Doctor W. W. Campbell (v. p. 12, ante), namely, that the path of the sun is to all intents and purposes rectilinear and that "the curvature of our path is undoubtedly so slight that we may consider it as a straight line for many generations of astronomers to come."

So minute is the range of the annual revolution of the earth around the sun relatively to the major orbit of the latter, that you can readily see why the earth's axis, year by year, should remain (almost) parallel to itself, the secret of our change of seasons. The slight departure from parallel that it does suffer in one such revolution is, of course, according to the new hypothesis, reflected in the alteration of the projected position of the Vertex on the precessional circle traced on the sky.

Observe, furthermore, that in revolving around the sun, the earth is obliged to pass twice each year across the precise line of his path, once in front of him, when she is on her way out, and the second time behind him, when she is on her way in. The first of these two points is known as the vernal equinox, and the second as the autumnal equinox. When the earth is at her outermost point, you will see that her Arctic region is pointed sunward, so that then we of the north have summer, while our winter solstice occurs when the earth is innermost.

In the very nature of things, with every star in movement, there is no such thing as an absolutely fixed point or an unalterable direction. Astronomers understand this (though strangely forgetful of it at times!) and do the best they can, under the circumstances, by choosing the most stable points and using them for reference. As 
important as any of these is what is known as the celestial vernal equinox, and it is that sky point where the center of the sun (to the eye of an observer supposed at the center of the earth) crosses the plane of the earth's equator. In my theory this occurs at the precise instant when the center of the earth, being then in advance of the sun, cuts across the line of his orbit. Every year the astronomers spare no pains to locate this point with all nicety possible, in order to keep accurate track of general changes of position.

It is important to remember that the celestial vernal equinox does not remain fixed with reference to the stars, but progresses westwardly around the zodiac. To understand why, you will readily perceive that in traveling around the great circuit and looking back once a year past the sun, just when the centers of both are on the orbit, the latter will be necessarily projected just as many degrees to the west on the celestial circumference as both have advanced in the same time in their common orbit. This annually executed are has been measured with the most refined nicety and has been found to be $50^{\prime \prime} .2$. In other words, the sun covers an are of his orbit $50^{\prime \prime} .2$ in length each year, and at this rate should complete one lap in 25,810 years.

\section{The Orbit and Velocity of the Sun Computed}

Inasmuch as, by hypothesis, the forward motion of the system is due to a definite and single force, to wit, the Prime Resultant, we might be justified in concluding, without further corroboration, that in one year the earth, from one vernal equinox to the next, advances along the main orbit exactly the diameter of her own-a conclusion which, were we to apply the principle of falling bodies, associating with it the principle of the lever, would prove generally true of all the rest of the planets. However, there is another chain of evidence to which I wish now to direct your attention which correlates these several data, namely: the velocity of the earth in her orbit, the length of the are, the degree of curvature of the are, the length 
of the diameter of the earth's orbit, the principle of falling bodies, the velocity of the sun as deduced from the direct study of the proper and the radial motions of the stars, and, finally, the parallactic motion of the Vertex.

Given the length of the arc of a circle in degrees and also in linear measure, it is only a matter of high-school mathematics to find the total length of the circumference and, from that, all the remaining functions of the circle. Again, if we know the length of an are and the amount it deviates from its tangent (technically called the curvature), we can, by a simple formula, ascertain the radius in the first instance, and so on to the rest. In the case under consideration we virtually know these two things: the arc's length, and its curvature in miles. The latter is quickly ascertained in this way:

If you have been following this somewhat intricate explanation closely, you should be able to see with little effort, that, inasmuch as the sun has at the instant of the second vernal equinox failed to get back fully into line with the same star it was in line with at the preceding equinox, the earth must continue on in her orbit beyond her equinoctial position until the old star, sun, and earth are all brought again into alignment. Astronomers tell us that it takes $20 \mathrm{~ms}$. 23 secs. for the earth to move far enough in her orbital journey to correct this discrepancy -this curvature of the arc. Now, the earth's average velocity being known (18.5 miles per second), to reduce this curvature to terms of miles we need only multiply this quantity by the number of seconds of time to obtain the answer, 22,625 miles.

As said before, given the length of are in seconds and the curvature in miles, it is easily possible to ascertain the radius of the circle. The rule, stated in the form of an equation with the initial letters of the things referred to (see Young's Gen'l Astr., Art. 420) is:

$$
\mathrm{c}: \mathrm{a}:: \mathrm{a}: 2 \mathrm{r}
$$

Now the length of the radius in terms of degrees of circumference is called a radian, and is known to be 
$57^{\circ} 17^{\prime} 44^{\prime \prime} .8$, or $206,264.8^{\prime \prime}$. Substituting this and our other values in the equation given, we have,

22,625 (miles) : arc (in miles) : : $50^{\prime \prime} .2: 412,530^{\prime \prime}$ which, being reduced, yields

$$
\text { arc }=185,977,500 \text { miles, }
$$

the meaning of which is, that in one year the center of mass of our solar system travels exactly the width of the earth's orbit, as measured along the equinoctial colure, AND THAT THIS COLURE MARKS THE LINE OF THE SUN'S PATH. Choosing round numbers, we ascertain by simple computation the length of the sun's orbit, considering it as a closed curve, to be $25,810 \times 186,000,000$ miles, or 4,800,$000,000,000$ miles, its diameter $1,530,000,000,000$; the axis of the cone from Centrum to Vertex 1,760,000,000,000 and from the Vertex to our sun (that is to say, the stem of the Prime Resultant) 1,918,000,000,000 miles. Finally, there being $31,557,000$ seconds in a year, the sun's velocity is found to be just a trifle short of six miles per second.

"But six miles", you will say, "is only about half the actual velocity of the sun as found by the direct methods, how do you account for the balance?" I will answer you. Hitherto in this discussion I have, for the sake of simplicity, ignored the very material feature that all this while the plane of the ecliptic, though remaining sensibly parallel with itself, is falling, keeping pace with the fall of the system. In other words, the sun is falling vertically just as fast as it is "gyroscoping" laterally; or, to borrow the parlance of the astrophysicists, he has both a "proper" motion and a "radial" motion. You may retort, that in such case he is travelling along the hypothenuse, hence can be going only about 8.5 miles per second. This is true enough, but considering the two velocities separately and then adding, which I understand to be the practice of astronomers, the total comes to 11.8 miles. In any case, the net result is so close to that obtained by the laborious practical methods that either result might fairly be cited in corroboration of the other. Besides, it ought not to be presumed that we have exhausted all the possible motions of the sun, and a sub- 
stantial margin of his absolute speed must logically be held in reserve, as it were, to admit of the introduction of possible new discoveries.

According to Newcomb, the pole moves 20" per annum on the scroll of the celestial sphere. Taking the sun's annual movement of 186,000,000 miles as the base line, the question arises as to how distant the Vertex must be to possess this parallax, or in other words, to make the distance 186,000,000 miles subtend an angle of only 20 seconds. To ascertain this we need simply divide the $186,000,000$ by 20 and multiply the quotient by the number of seconds in a radian, yielding as a result, 1,918,$000,000,000$ miles, which is precisely the same value we found by the other process.

Again, it may be interposed that if, indeed, the system be falling at the rate specified, it would in the precessional period fall a sheer height equivalent to the full length of the sun's orbit-practically twice as low as the Vertex. This is precisely what I contend. But the Vertex is no more riveted to one spot than a horse drawing a vehicle. It is merely the crossing place of gravitational strands that, real and powerful though they are, are yet as imponderable, as elusive, as mobile as the rays of light itself. The Vertex, in fine, is a wili-o'-the-wisp, beckoning the system on and on forever, and, indeed, the Gravisphere itself should be conceived of as in the process of falling in an enormous spiral curve on the order of a hundred trillions of miles in diameter and a coil period of some two millions of years.

If the reader has acquired the impression that I identify the Vertex with the center of the stellar universe, he is mistaken. There may be such a center, perhaps; but if so, it is as shifting as the clouds, since all things are in motion. The Vertex is for the system a proximate center only, just as the earth is for the meteors that fall in upon it. The garden of the sky, indeed, is crowded with these umbels of forces carrying, perched on their stems, their planetary efflorescences. Beyond that Vertex is another far more distant, beyond that again, another 
more distant still, and so on, rising higher and higher in scale, and bearing upon their twigs systems, and upon their branches systems of systems of worlds. To my mind, the flatness and the well-proved depth of the disc of the Milky Way eloquently testify that it is a gravitational unit in a mightier invisible universe, invisible only because the light of its component stars is drowned in the vast sea of space. As such a unit, of course, the Galaxy should rotate around an axis in search of equilibrium, and it appears that astronomers have already detected some indications of such rotation! Underlying the whole structural scheme of the macrocosm, as under the humblest habitation of man, lies the great principle that Newton took such pains to eliminate-EQUILIBRIUM.

\section{The Obliquity of the Ecliptic}

Inter-related with this problem of precession is that of the change in the obliquity of the ecliptic. To quote from Young again (Genl. Astr., p. 137): "The ecliptic slightly and very slowly shifts its position among the stars, thus altering the latitudes of the stars and the angle between the ecliptic and the equator, i. e., the obliquity of the ecliptic. This obliquity is at present about $24^{\prime}$ less than it was 2,000 years ago, and is still decreasing about half a second a year. It is computed that this diminution will continue for about 15,000 years, reducing the obliquity to $2214^{\circ}$, when it will begin to increase. The whole change, according to J. Herschel, can never exceed about $1^{\circ} 20^{\prime}$ on each side of the mean."

The explanation of this phenomenon is not far to seek. We have only to assume the plane. of the gravAntarctic Circle to constitute the "invariable" plane of the solar system and to picture the oval of the earth's orbit as, so to speak, balanced on the rim of this Circle in such a way as constantly to preserve to it an inclination of $1^{\circ} 20^{\prime}$ throughout the 260 centuries that it takes for it to "slide" around the precessional circle. Or, to put the matter from another point of view, the revolution of the ecliptic around the solar orbit causes its pole (the Ver- 
tex) to project itself upon the celestial sphere in the shape of a minute precessional circle approximately $2^{\circ} 40^{\prime}$ in diameter, corresponding as to period and direction of rotation precisely with the precessional circle described by the gravitational pole.

In nature, however, nothing is absolutely fixed, but only relatively, and the so-called invariable plane is by no means an exception to the general rule. The charting of the precessional circle (see Flammarion's Popular Astronomy, p. 40), shows that its center is not stationary, but is heading, apparently, for the space between Hercules and Lyra. This phenomenon I attribute to a rotation of the Gravisphere, not, however, around a stationary axis, but because of its revolution in an enormous orbit (spiral, of course) whose definite curvature, period and velocity of description remain to be ascertained. If I may venture upon a rough approximation, I should judge the radius of the curve to be not less than ten light years and the period upward of $2,000,000$ years, with a maximum limit of, say, 20 and 10,000,000 respectively.

\section{The Rotation of the Apsides}

Another radical reform in mathematical astronomy made necessary by the discovery of the spiral as opposed to the rectilinear conception of the sun's path, concerns the problem of the rotations of the lines of apsides. Astronomers recognize three kinds of years, namely, (1) the tropical year, from vernal equinox to vernal equinox, (2) the sidereal year, from the time the sun leaves a given star's meridian until he returns to it again, and, (3) the anomalistic year, from one perihelion place to the next. Of these, the first is 365 d. 5 h. 48 m. $45.98 \mathrm{~s}$. long, the second $20 \mathrm{~m} .23 \mathrm{~s}$. longer, and the third longer still by $4 \mathrm{~m} .39 \mathrm{~s}$. Now, we have previously seen that the reason for the sidereal year being longer than the tropical year is because, while the earth is rolling forward in her orbit, the system as a whole is slipping backward, around a circle, the completion of which requires 26,000 years. The result of this arrangement is to cause the earth an 
annual loss in position with respect to the stars of $50^{\prime \prime} .2$; and since the anomalistic year is longer by $12^{\prime \prime}$ of arc than even the sidereal one, it follows that the perihelion advances, not merely $12^{\prime \prime}$, but $12^{\prime \prime}+50^{\prime \prime} .2$ annually. In short, the time of the revolution of the earth's apsides is not the apparent period of 108,000 years, as till now supposed, but

$$
\frac{360^{\circ}}{62^{\prime \prime} .2}=20,900 \text { years (about) }
$$

The periods of the other planets, to be derived by the same method, should therefore hereafter form our bascs of comparison and correlation. In passing, be it noted, one consequence of this interpretation is to take Venus out of the category of exceptions (in which it has stood alone) and include it in the general rule, namely, that the perihelia of all the planets advance.

\section{The Star Streams So-Called}

The reader may infer from what has just been discussed that the star streams that Kapteyn was the first to call attention to, may find their explanation in this principle. Such is not my own opinion. This phenomenon of star-streaming is, like the daily motion of the heavens around our earth, illusory. It is only apparent. It arises from a misguided attempt on the part of astronomers to generalize from data gained by observing from one moving body another moving body with reference to a third moving body, all of whose velocities and distances, relative and absolute, are highly conjectural, and, in the case of the earth at least, misconstrued. No! whoever cares to play the role of skipper of this good ship, Earth, and in imagination will take his stand at her south pole and sail her around the sun's orbit a voyage or two, watching the while the stars on shore, will soon come to realize that the supposed streams are but the optical effect of our change of position. 


\section{VI}

\section{THE TIDES}

$7 \mathrm{HE}$ ebb and flow of the tides is altogether too conspicuous and important a phenomenon of nature to have escaped the notice and observation of the ancients, and long before Newton's day it was shrewdly suspected that some sort of causal relationship subsists between the movements of the moon on the one hand and the behavior of the tides on the other. By the time Newton arrived on the scene, this suspicion had developed into a general scientific conviction, although no explanation had yet been suggested. The circumstantial evidence on which this conviction is founded may be briefly summed up as follows :

Besides her apparent diurnal revolution around the earth from east to west, the moon has a real motion around our planet in the opposite direction, from west to east, a circuit which she completes (from new moon to new moon) in about $291 / 2$ days. On account of this latter motion, the moon's time of rising is delayed from one day to another by an average period of 51 minutes. Now, the strange part of it is that high tide arrives at any given port by this same interval of 51 minutes later each successive day, and the natural inference arises that the moon is somehow the cause, and the tide the effect. But the coincidence extends even farther, for it has been demonstrated that the height of the tide varies rhythmically with the moon's changes of phase, being highest at the syzygies and lowest at quadrature. Furthermore, the tide is higher by about 20 per cent when the moon is at perigee than when at apogee. 
Newton himself was so completely persuaded by this evidence of the moon's being the mother of the tides that it never occurred to him, nor indeed does it seem to have occurred to anyone but the present writer, to cast about for any other solution. That a new solution is imperatively demanded, however, is rendered certain by the candid confessions of the Newtonians themselves, who, after two centuries of ceaseless striving to fit their theory to nature, have been reluctantly constrained (v. p. 18) to testify that the two are "utterly inreconcilable".

It is but one of many glaring inconsistencies of modern astronomical science that, in spite of these and an unlimited number of other such damning admissions of the objective falsity of Newton's tidal theory, our scientists nevertheless continue to teach it in the schools as one of the great scientific gospels. All the so-called modern cosmogonies-the Planetesimal hypothesis of Chamberlin and Moulton, Arrhenius' theory of LightPressure, See's hypothesis of a Resisting Medium, and the rest,--are based in whole or in part on this admittedly false doctrine. Indeed, in the very book in which he gave utterance to the paragraphs just quoted, Darwin goes on and elaborates his grotesque theory of Tidal Evolution, of which, in concluding, he himself speaks in these slighting words (The Tides, p. 284) :

There is nothing to tell us whether this theory affords the true explanation of the birth of the moon, and I say that it is only a wild speculation incapable of verification.

To cap the climax of absurdity, the article Tides in the new Britannica was contributed by this same Darwin, and it is given up almost wholly to the exploitation of this "wild speculation incapable of verification" to the exclusion of virtually all other pertinent matter! What a travesty is this on science! Here we have, in chronological order: first, Newton's theory; second, universal admission of its unsoundness; third, a complicated elaboration of the theory by one who had already condemned it; fourth, condemnation of the elaboration by its own author; and fifth, the memorializing of the author and 
his "wild speculation" in the chief publication of the world!

\section{NEWTON'S THEORY OF THE TIDES}

Inasmuch as the tides rise it was natural enough for Newton to surmise, in the first instance, that they are caused by gravitation lifting the waters away from the earth proper; and inasmuch, also, as there exists a coincidence between the moon's schedule and the schedule of the tides, it was not less natural for him to infer, at least provisionally, that the moon, rather than the sun, is the primary tidal force. Accordingly, he conceived the moon as drawing the oceans immediately under her away from the earth's solid part or kernel, and the kernel in turn away from the nether oceans, thus causing the double tide that observation reveals to exist.

When he came to compute the relative attractions of the sun and moon, however, he found that, after making due allowance for their respective masses and distances, the attraction of the former upon our earth is 180 times stronger than the lunar attraction. Such a result was, of course, incompatible with his primary hypothesis that the moon is the greater tidal force (a hypothesis which, alas! he took to be axiomatic), so he cast about in his mind for a way to reverse the order of their potency, and to twist things to fit.

Continuing his argument, he reasoned that the waters that actually compose the tidal hillock are to be differentiated from the level-mass of the ocean in this, that they are the part displaced, having to be drawn in from the surrounding regions. Such being the case, the attraction of the moon which drew them in could not have been, at first, vertically exerted upon them, but obliquely, and the same reasoning applied equally to the sun's attraction. It consequently followed, mathematically, that the tidal forces vary, not as the inverse squares (the law of gravitation) of the distances of the sun and the moon, but as their inverse cubes. Computing anew on this basis Newton made out the moon to be about four times 
stronger, tidally, than the sun, instead of 180 times feebler.

Observation, from Newton's day to this, has failed to reveal any signs of a separate double solar tide, which theory indicates should exist. According to modern computations, the ratio of the two tidal forces is, roughly, two to one instead of four to one; hence the academical argument for the visibility of such a separate solar tide looms stronger to-day than it appeared in Newton's age. He sought to explain the physical absence of this tide by postulating a merger of the solar with the lunar tide, at one time augmenting and at another offsetting it, according to the relative angular positions of the two bodies with respect to the earth.

Now, although it is quite true that there is a startling coincidence between the measure of the daily advance of the moon eastwardly in her orbit and the interval between the arrival of successive high tides at any given port51 minutes in both cases-there is a no less startling discrepancy as to the meridianal places of the moon and high tide at any given moment. According to the main hypothesis, high tide should occur immediately under the moon; for instance, when the moon is on the meridian of New York, high tide should then be filling that city's harbor. Instead, we find that when this latter event occurs the moon has preceded it by about eight hours, and is already some thirty degrees below New York's western horizon! Why this great hiatus between cause and effect? Newton claims it is due to the "dragging" of the tides, arising from the presence of interposing land masses, friction on the ocean beds, and the inertia of the water itself.

The assumption that the moon draws the oceans away from the earth's solid part clearly implies a superiority in the degree of the intensity of her attraction upon the oceans, as contrasted with her pull on the kernel, and a relative holding back of the latter. Newton knew as certainly, although not so accurately as we, that the density of the earth's solid part is several times greater than that of water and that, by the strict law of mass, the 
kernel, notwithstanding its slightly greater distance from the moon, should be attracted much more powerfully than the oceans in front of it. He knew, also, that the resultant effect of such action could only be to shallow the seas on the earth's moonward side instead of deepening (raising) them. This conclusion, however, did not suit Newton's preconceptions in the least, for it meant retracting his previous reasoning, restoring the original, to him obnoxious, ratio of 180 to 1 in the sun's favor, and, in short, relinquishing altogether his cherished theory of the lunar causation of tides. Unequal to this sacrifice, he sought a way out of the dilemma by tampering again with his own law of gravitation. As one lie leads to another, so one basic misinterpretation of nature's laws leads to an endless chain of absurdities. He had already dared to distort the second clause of the law to read that tidal forces vary, not as the inverse squares but as the inverse cubes of the distances, hence he now felt driven, in the interest of consistency, to go on and alter the first clause to match its changed companion. Accordingly, he made that to read, that gravitational attraction, when operating as a tidal force, disregards differentiations of density, and here it was that he introduced in support his famous vacuum-tube experiment which we discussed in the preceding chapter.

Newton's next step was to invent some method, favorable to his theory, for computing the tidal heights. Making use of his rule of inverse cubes, he ascertained the tidal force of the moon to be $1-2,871,400$ of the earth's gravity and that of the sun, 1-12,868,200. (Young gives 1-8,640,000 and 1-19,600,000 respectively). The question with him was: How are these quantities to be translated into terms of tidal height so as to obtain plausible results?

It was well-known to the generation before Newton that the figure of the earth is not that of a sphere, but of an oblate spheroid, and her equatorial ring seems always to have been assumed, as a plain matter of course, to be the running effect of the centrifugal force of her axial 
rotation. This view was blindly adopted by Newton himself, and is the one universally acquiesced in to-day by all recognized scientists. Some skeptical lay readers may infer that the ring, to some extent at least, may be the cumulative effect of the long past, but let me assure him solemnly that this is not the orthodox interpretation. In the time of Newton the thickness of this ring at the equator was supposed to be 85,472 Paris feet (a Paris foot being about 1-15 longer than the American standard), but the modern estimate is only $13-\mathrm{r} / 2$ miles (71,280 feet).

By a combined process of pendulum experimenting and computation, Newton arrived at the conclusion that the centrifugal force of the earth's rotation at the equator serves to lighten any given body at that place by 1-28. of the weight it would have were the globe at rest; that is to say, the centrifugal force there is equal to 1-289 of gravity. Continuing, he reasoned that, inasmuch as this force, being equal to 1-289 of gravity, sufficies to elevate her equatorial regions by 85,472 feet, the attraction of the sun, which is $1-12,868,220$ the strength of gravity, should be able to elevate those regions by 289 times 85,472 feet, or $24,701,408$ feet divided by $12,868,200$, or very nearly 2 feet. But let me quote his own words (Book III, Prop. 36, Principia) :

Cor. Since the centrifugal force of the parts of the earth, arising from the earth's diurnal motion, which is to the force of gravity as I to 289 , raises the waters under the equator to a height exceeding that under the poles by 85,472 Paris feet, as above, in prop. I9, the force of the sun, which we have now shewed to be to the force of gravity as I to $12,868,200$, and therefore is to that centrifugal force as 289 to $12,868,200$, or as I to 44,527 , will be able to raise the waters in the places directly under and directly opposed to the sun to a height exceeding that in the places which are 90 degrees removed from the sun only by one Paris foot and II-I/30 inches, for this measure is to the measure of 85,472 feet as I to 44,517 .

At this stage Newton appears to have rested his case, but not so his successors, who have had the desperate courage to pursue his fatal logic further, even to the bitter extreme of swallowing the inescapable reductio ad absurdum that neither the moon or the sun is the dynami- 
cal cause of the tides, but that the centrifugal force of the earth's axial rotation is! Nor, granting the premises laid down by their leader, can one find any fault with their logic, as such. Suppose, say they, that the earth did not rotate on its axis at all, then there would be no centrifugal force and, by the same token, there could be no equatorial ring and, incidentally, no tide. Test the matter for yourself: Substitute in the Corollary zero wherever the quantity 85,472 feet appears and you will find that, no matter how stupendous the tidal forces of the sun and moon might otherwise figure out, Newton's odd method of computing would, did the planet not rotate, inevitably reduce them all to nothing. Here is what no less a personage than the late lamented Sir Robert Stawell Ball, the most famous English-speaking astronomer of our day, says on this subject (Story of the Heavens, p. 539) :

The tides are, however, doing work of one kind or another. A tide in a river estuary will sometimes scour away a bank and carry its materials elsewhere. We have here work done and energy consumed, just as much as if the same task had been accomplished by engineers directing the powerful arms of navies. We know that work cannot be done without the consumption of energy in some of its forms; whence, then, comes the energy which supplies the power of the tides? At a first glance, the answer to this question seems a very obvious one. Have we not said that the tides are caused by the moon? And must not the energy, therefore, be derived from the moon? This seems plain enough, but, unfortunately it is not true. It is one of those cases, by no means infrequent in Dynamics, where the truth is widely different from that which seems to be the case. An illustration, perhaps, will make the matter clearer. When a rifle is fired, it is the finger of the rifleman that pulls the trigger; but are we, then, to say that the energy by which the bullet has been driven off has been supplied by the rifleman? Certainly not; the energy is, of course, due to the gunpowder, and all the rifleman did was to provide the means by which the energy stored up in the powder could be liberated. To a certain extent we may compare this with the tidal problem; the tides raised by the moon are the originating cause whereby a certain store of energy is drawn upon and applied to do such work as the tides are competent to perform. This store of energy, strange to say, does not lie in the moon; it is in the earth itself. Indeed it is extremely remarkable that the moon actually gains energy from the tides by itself absorbing some of 
the store which exists in the earth. This is not put forward as an obvious result, it depends upon a refined dynamical theorem.

We must clearly understand the nature of this mighty store of energy from which the tides draw their power and on which the moon is permitted to make large and incessant drafts. Let us see in what sense the earth is said to possess a store of energy. We know that the earth rotates on its axis once every day. It is this rotation which is the source of the energy. Let us compare the rotation of the earth with the rotation of the fly-wheel belonging to a steam engine. The rotation of the fly-wheel is really a reservoir, into which the engine pours energy at each stroke of the piston. The various machines in the mill, worked by the engine, merely draw upon the store of the energy accumulated in the fly-wheel. The earth may be likened to a gigantic flywheel detached from the engine though still connected with the machines in the mill. From its stupendous dimensions and from its rapid velocity, that great fly-wheel possesses an enormous store of energy which must be expended before the fly-wheel comes to rest. Hence it is that, though the tides are caused by the moon, yet the energy they require is obtained by simply appropriating some of the vast supply available from the rotation of the earth.

It is quite true that many astronomers, without, however, making any pretense of logical demonstration, hypothesize the existence of a statical tide, that is to say, a tide on a non-rotating body. Standing by itself, this is an eminently sensible idea; but, taken in connection with Newton's process of computing it, it is a wholly unwarranted assumption. Besides, does not Young say, "In fact the statical theory becomes utterly unsatisfactory in regard to what actually takes place"?

Boiled down to its dregs, then, this is Newton's Tidal Theory:

1. The moon is the primary cause of the tides, and the sun the secondary.

2. The actual, or dynamical, cause of the tides is, nevertheless, neither sun nor moon, but the centrifugal force of the earth's axial rotation. In fact, the moon even feeds upon the energy of this rotation.

3. The elevation known as the earth's equatorial protuberance is the running effect of that centrifugal force. 
4. The tides are likewise the running effect of this same centrifugal force.

5. This centrifugal force exists of itself without any pre-existing cause.

6. There is no way by which this force is replenished. The earth is on the order of "a fly-wheel detached from its engine". What sort of an engine it was we cannot say, but we do know that it is not attached and operating now.

7. To sustain in place the equatorial ring, this centrifugal force continuously expends out of its stored energy 1-289 of the power of gravity, and, to produce the lunar tides, 289-2,811,400 of gravity.

8. Notwithstanding this lack of an engine and these dissipations of energy, the centrifugal force remains intact forever.

9. The explanation of this mystery is that the centrifugal force is persistent in character, this word having been suggested by Spencer as an improvement on Newton's word inertial. The discovery of this synonym is esteemed one of the great triumphs of cosmic science.

10. The moon's tidal force is employed in dragging the tides from where the major premiss of the theory says they are generated and ought to appear, to exactly those places where the same theory says they are out of place and ought not to be.

11. The theory is based on the law of gravitation by converting the clause that reads "varies directly as the product of their masses" into "varies regardless of their densities," and the clause "inversely as the squares of their distances," into "inversely as the cubes of their distances."

12. It denies the law and principle of equilibrium in its application to cosmic bodies.

13. Weighed by the physical facts "it is nearly as much wrong as possible."

14. It is one of the fundamental gospels of cosmological science. 


\section{Newton's Theory Criticised}

Since the death of Newton in 1727, the accumulation of scientific data has proceeded with Brobdignagian strides, and this is true of astronomy no less than of the other physical sciences. Linnaeus, the father of modern botany, was only twenty when Newton died; Dalton, the originator of atomic chemistry, was not born until nearly forty years later, 1766; Cuvier, the founder of systematic zoölogy, in 1769; Charles Darwin, in 1809. These celebrated names imply that there is more for man to do than merely to acquire stores of isolated items of information; they connote classification and generalization of these data into systems that not only broaden our concepts, but supply keys to much that remains unknown.

Now, if the fundamental truths were the first to come into our knowledge, the building up of a science would be a comparatively simple matter. But they by no means do so. Even so, little harm would come of it, were it not for the proneness of all of us to think and act mechanically, along conventional lines, and to look with disfavor and often with contempt on the "crank" who presumes to set his opinions up against those of the reactionary majority. Since Newton's day, numerous such fundamental truths in astronomy have come to light that our scientists have been treating as minor and tacking here and there onto the most convenient niches in the superstructure, instead of courageously razing, as they should, the ramshackle structure to the bed rock and rebuilding solidly with free hand and fresh initative.

Who among us, I ask, gifted with ordinary intelligence, a heart of perseverance, sufficient leisure, an overpowering love for and interest in the subject, and means of access to the great stores of modern data, should not be capable of constructing de novo a better system of cosmology than Newton or any other man or genius could be expected to do in a day when such basic indispensable truths as the following were unknown and unsuspected?

1. The age of the earth. Newton conceived it as especially created for man only 6000 years ago, and that, 
in another paltry millennium or so, it is doomed to perish in flame and ashes. This notion of the earth's transiency narrowed his outlook on the greater universe most pathetically, insomuch that, in all his deliberations, he overlooked altogether the relations of the solar system to the stars, and treated it as a universe unto itself. He did not know of the sun's motion, or that the stars move in regular courses. (3) He took no account of the greatest dynamical factor in nature, namely, the composite of the stellar attractions. (4) He never heard of the existence of nebulæ, for these were not discovered by Sir Wm. Herschel until two score years after Newton's death, hence the characteristic forms of these wonderful objects carried no suggestion to him. (5) He knew of the existence of only six great planets. Had he known of the asteroids, he might have thought twice before predicating divine agency to set these, like the great planets, in what he conceived to be miraculous and studied motion. (6) He knew of the secular acceleration of the moon, but he did not foresee that mathematics alone would never solve it. (7) The problem of the origin of the sun's heat troubled him not, for to him it was transient as the earth. (8) He had no ideas whatever about the genesis of new stars, or about their variability. (9) The use of steam as a source of mechanical power was unknown, and, of course, so was the mechanical theory of heat as well. (10) Spectrum analysis had not yet demonstrated that the sun and stars are essentially of the same chemical composition as our base earth. (11) Finally, Newton lacked the advantage we possess of factual knowledge that his tidal theory is all wrong. Had he had this knowledge he might have taken a second thought and, retracing his steps, hit upon the true solution. After severally weighing this formidable array of his handicaps, ask yourself whether the presumption should be in favor of, or against, Newton's theory of tides or, for that matter, any of his theories, and what should be our duty in the premises?

It is curious that our Newtonian friends, who lay such great store upon the value of the concordance of 
moon and tide in the single particular that each has the same habit of arriving at any given port 51 minutes later each succeeding day, pedal most softly on the dissonance between the meridianal places where these phenomena respectively appear at the same instant of time. According to Newton's main hypothesis, the tidal crest should exist at all times nowhere but exactly beneath the moon. Were this, indeed, the case, the causal relationship between moon and tide would be all but conclusively demonstrated. But the uncompromising fact is that the tide which the moon is alleged to raise up under her, never by any chance appears anywhere near there, but invariably hides below her horizon some eight hours in her wake!

Having, let us say, been freshly taught by our Newtonian savants as to how the moon causes the tides, and, furthermore, that gravitation acts instantly over very great distances, our first impulse is to glance at the nearest expanse of ocean to note the evidential phenomena. But instead of beholding the expected hillock, we really see a hollow! Dumfounded, we conjure up apologies for the solecism, as, that the water here is too shallow, or the expanse too restricted, and the like. Accordingly, we decide to test out the hypothesis under the most favorable conditions possible, and for this purpose select the central meridian of the Pacific, on the equator, where the ocean is at its deepest, when the full moon is in the zenith, and where there can be no suggestion of continental resistances to hinder the tidal process. But even here, here, under ideal conditions, we behold, not a rise, but a more pronounced depression than ever! and we recall Darwin's plaint once more, "It would seem, then, as if the tidal action of the moon was actually to repel the water instead of attracting it; and we are driven to ask whether this result can possibly be consistent with the theory of universal gravitation!

Now the tide must come into being somewhere and somewhen, and it must exist before it can be "dragged". If it be not lifted into being under the ideal conditions 
mentioned above, when or where else can it be generated with greater certainty and facility, or more promptly? Again, if it arise not there until many hours after the moon has passed, how can she drag thence against resistance what she could not and did not, lift, unresisted, on the spot? While, amazedly, we are pondering these contradictions, our Newtonian cicerone pulls us by the sleeve and learnedly says, "The tide never forms where it is created, save only in lofty scientific contemplation; but look away off into the mid-Atlantic. There is the tide," he explains, "that the moon gave birth to yesterday while here over the Pacific; but, in the meantime, she has dragged it thence overnight around the curve of the globe. Eight hours hence, when the moon, now in her zenith, has descended 30 degrees below our western horizon and is soaring over India", he goes on, "the crest of the tide will arrive here in the midst of the Pacific, and we shall speak of it as this day's tide." Ask him at what meridian yesterday's tide dies out and to-day's begins; whether the tide of the Atlantic dissipates itself by contact with the eastern shores of the American continent, or is dragged around Cape Horn into the eastern Pacific; whether, if it be thus dissipated, the Pacific tide is not also dissipated upon the eastern shores of Asia, India and Africa and never enters the Atlantic at all-and he will answer you somewhat as Darwin does, "It is interesting to reflect that our tides to-day depend even more on what occurred yesterday or the day before in the Southern Pacific and Indian oceans than on the direct action of the moon to-day-the problem is one of insoluble mystery."

Again, according to Newton, the terrestrial waters located on the moonward side flow toward that body, while the waters on the nether side tend toward the lunar antapex. Now, the crests of all the tides at all times are invariably located below the moon's horizon, consequently they belong to the nether hemisphere and, by premiss, should seek the antapex. But observation shows otherwise, for the main tide keeps coursing up the earth's side incessantly, seemingly determined to rise into the moonlit 
hemisphere,-a feat, however, which it never achieves. In short, whether we regard the tide as part of the moonlit hemisphere or as part of the other, its behavior is equally contradictory of the Newtonian hypothesis.

Only a little insight is required to reveal that Newton pictures the moon as doing and undoing its alleged work at one and the same time-a house divided against itself. Could you look down from the moon you would see no sign of the tide, for the crest of it is perennially hidden from her sight and even some thirty degrees around the bend of her horizon. Now let me ask you to translate yourself in spirit to that point in the moon's orbit diametrically opposite her, and thence to look down upon that side of the earth. According to Newton's primary hypothesis, where ought you to find high water? Why, at the point vertically under you, in the very center of the disc. But where do you really find it? I answer you, along the marginal rim of the disc. Strange contradiction! Here we are told that the moon's function is to gather the waters to the places under and opposite her, and in the next breath we are solemnly informed that by virtue of that same power she foils herself and drags those waters away from herself to the places most distant from those central spots, and what is more, perversely keeps them away! According to theory, the tidal crest should not roll toward the rim as it does, but toward the center or antapex, which in fact it really FLEes. Inconstant moon! Bringing to bear her full lifting power directly upon the upturned sea, with no resistance to counteract her, all she can do is to indent the surface! With everything against her-continental resistances, the curvature of the earth, even her own contrary pullshe drags the tide after her without a slip or a break! Every process of dragging against resistance implies retardation in geometrical ratio: how comes it, then, that after, through this cause, having fallen behind the moon 120 degrees, the tidal crest thereafter eternally holds its full integrity and speed, instead of softly diffusing into a neutral sheet? 
We are informed that the moon causes a double tide, that is to say, one tide on the side of the earth that faces her, and a secondary tide on the earth's face hidden from her. The first of these is understood to be the tidal crest which follows the moon by the interval of about eight hours (120 degrees). For brevity, let us call this the "follow" tide. Assuming that the secondary tide is normally diametrically opposite this, or 180 degrees behind, the former should in fact be just 60 degrees in front of the moon's position; hence we may, for convenience, call this secondary tide the "leader." Now, if there is any merit in the conceit that the moon makes a business of dragging tides around, why does she not lasso the leader tide, where it rolls less than a quadrant ahead, and drag it back to her, thereby fulfilling the major hypothesis? This argument, in itself so reasonable, is made stronger by the circumstance that, whereas the follow tide, in its effort to reach the moon, is obstructed by the frictional resistances conjured up by Newton, the same sort of resistances rising up in the path of the leader tide ought to abet the moon's effort to catch up, and drive that tide back into her corralling arms.

It will scarcely be disputed that the tidal elevation is necessarily subject to a continuous levelling or wasting process, tending toward the general smoothing over of the ocean surface, and that, in order that the tide may persist, it must be continuously recuperated. Such restorations must, perforce, occur on the spot, not thousands of miles away. Between the Cape Verdes and the Hawaii Islands stretch about 120 degrees of longitude, so that when the moon soars over the latter, the tide washes the shores of the former. By what dark magic does the moon thus ventriloquize her tidal mandate from where she stands at work, back around the bow of the earth, to the eastern Atlantic? A straight line drawn from the moon at any time to the tidal crest would pierce the earth hundreds of miles below the surface, and it is plainly to be seen that the attraction of the moon, acting directly along such a line, will attack the tide through the earth 
from underneath, and should tend to level, not to augment its height. The Newtonians, however, in their vaunted wisdom, picture the attraction of the satellite as turning the curve of the earth, as round a pulley, and laying a prehensile hand upon the tide and hauling it forward by main force.

Newton's idea of how the tides are formed, namely, by the drawing away of the nearer waters from the kernel, and the latter in turn from the rearward waters, contemplates, as its vital feature, an actual physical displacement of all three in the line of the moon's radius vector. Let us see just what such possible displacements would mathematically amount to and whether or not they measure up to the requirements.

According to the principle of gravitation, any object, for example an apple, falling earthward, attracts as much at it is attracted, for, viewed from either end, the tractive tension is identically the same. This does not signify, however, that in coming together earth and apple will meet half way, but rather that they will traverse distances inversely proportional to the square roots of their masses. A while back, we saw that the moon has been computed to fall 1-19 of an inch per second. Since, however, she is only 1-81 of the earth's size, the latter should theoretically fall moonward only 1-171 of an inch in the same brief period. This computation, be it noted, is based on the original law of the inverse squares, which Newton repudiates in favor of his improvised rule of inverse cubes. Adopting his rule, we shall have to reduce our already small fraction by multiplying the denominator by 60 (the moon's distance being 60 times the earth's radius), whence we derive the quantity 1-10260 of an inch as the measure of the lunar tidal deflection of the earth per second. But this quantity, again, must be halved, for the reason that there are hypothetically two tides, fore and aft, each of which must be allowed an equal share of the provided space, yielding us only 1-20,520 of an inch for each. Summing it all up, Newton's conception contemplates that the moon by drawing the 
oceans on the near side away from the earth's kernel the infinitesimal fraction of 1-20,520 of an inch per second, at once creates and provides room for a tide $81 / 2$ feet high, or more than 2,000,000 times its own magnitude! Doubtless some will retort that the tide is the work of a day and not merely of a second. Very well, grant even this for the sake of argument and say the effect is cumulative for a full day and thereafter remains uniform. In a day there are 86,400 seconds: multiply this number into $1-20,520$ of an inch and you get, even with this improvident concession, the paltry space of only $4 \frac{1}{2}$ inches, or 22 times less than what the case requires. For the tide to reach the high altitude of $81 / 2$ feet that Newton gave it, the tidal force would have to accumulate for nearly a month! To my mind, however, it is quite as preposterous to suppose such cumulativeness to take place for the period of a day as for a year, and I hold that the tidal force and the tidal effect are mutually commensurate from one second to another, and that it is for each individual second to tell its own tale. To get an idea of the woful inadequacy of this ratio of 1 to $2,000,000$, then, the reader may mentally compare a tub of water to Niagara's overflow in the space of a full minute! To just this extent does Newton's hypothesis fall short of the actual dynamical requirements.

In making his computations of the tidal heights, even on his own theory, Newton committed two serious oversights. The first of these was in erroneously treating the entire thickness of the equatorial ring as being centrifugalized instead of only one-half of it. The other half of the ring is, of course, rightfully in place, being a part of the original ideal spherical shape. For it should be plain to any person of intelligence that subtracting a unit from one of two equal quantities and adding that unit to the other quantity will make a difference of two units, and not merely of one. Accepting as correct the estimate of the earth's polar diameter at 7,899 miles, and of the equatorial at 7,926 miles, given out by the National Geographical Society, the disparity between the two is 27 miles. Were the earth molded into a perfect sphere, the terres- 
trial diameter would be uniformly 7912.5 miles. To deform the earth, then, into its present shape required the centrifugalizing to the equatorial belt of $13 \frac{1}{2}$ miles of thickness from the polar axis. But we must not overlook the circumstance that this excess matter is evenly distributed all around the equator, consequently the thickness of the ring at any given point exactly on the equator though $131 / 2$ miles, the part centrifugalized is just $63 / 4$ miles, or only 35,640 feet as compared with the 85,472 feet adopted by Newton. Had Newton done what rightly he should have done, and divided his quantity by two, he would have reduced his tidal heights for sun and moon to about $51 / 2$ inches and 4 feet 4 inches, respectively, and, on the like supposition, Flammarion's estimates would fall to 4.7 and 9.9 inches.

Newton's second blunder lay in assuming that the entire equatorial ring, the whole 13.5 miles, is composed exclusively of water, though he could not help but know that not more than one-sixth of it is so constituted. A strict regard for accuracy should have prompted him to allow for the probability that the land masses and the rocky bed of the ocean, which together make up the other five-sixths, are upheld not by centrifugal force at all but by their solid supports. As a matter of fact, however, he made no such allowance, nor do his successors to this day, nor has anyone in their behalf attempted to offer any apology for the omission. The reason is plain enough, for to do so would be to render their estimates too ridiculously trivial for consideration, namely, Newton's, 1 inch and 9 inches, and Flammarion's, .8 and 1.8 inches respectively.

Although astronomers, as a class, openly profess to subscribe to every jot and tittle of Newton's tidal theory down to the final deductions that non-rotating bodies cannot bear tides and that the axial rotation of the earth supplies the power for the terrestrial tides, yet such is the saving power of common sense over abstract theory that these same astronomers flirt with the heretical notion of the possibility of the existence of a statical tide on non- 
rotating bodies. However, this new notion may be rendered compatible by employing another method than that of Newton to compute the tidal heights, a method, too, that on the face of it is decidedly more logical in every way, for it not only permits of the postulation of a statical tide, but also shifts the task of producing the tides in general from the centrifugal force of the earth to the moon's attraction, where Newton initially meant to place the credit.

According to Sir John Murray, the average depth of the oceans is 12,480 feet, or about 149,760 inches. Let us, if you please, consider this water as a prize being wrestled for by the earth, on the one hand, seeking to maintain it in a quiescent state of equilibrium, and the moon, on the other, striving to capture it from her. As we have already seen, Newton estimated the moon's tidal force as only 1-2,871,400 of gravity. To get a concrete basis of comparison between these two forces, then, in terms of ocean depth, all we need do is to divide 149,760 inches by 2,871,470; giving for a result approximately one-twentieth of an inch, or about the thickness of shoe leather. Of course, this result is ridiculously out of proportion to the phenomena to be explained, but let the blame fall on the theory, where it belongs, and not on my method of computing it, which is logically sound. The average daily rainfall for the whole earth is almost exactly one-tenth of an inch. Assuming the average daily evaporation from the surface of the sea to be the same, there looms out the astounding reductio ad absurdum that, on Newton's own showing, the sun's evaporating effect is just twice as great as the moon's tidal force! Besides, the sun raises his burden clear to the clouds.

Most of my readers, I dare say, imagine, with Sir Robert Ball, the earth to be a body so enormous, and the energy of its axial rotation so immeasurably great, that the theoretical drains scientists impose upon it are relatively too trivial to cut any material figure. Suppose we investigate this matter in the spirit of the Missourian and let us first take up the factor of the dynamical sustentation of the equatorial ring. 
You cannot have forgotten our former experiment of whirling the ball at the end of the string, and how I tried to make clear the fundamental distinction between the principle of that experiment, embracing as it does a "flinging motion" outward from the center, and the case of the earth and moon, in which such a flinging motion is conspicuous by its absence.

That instance has a parallel. The experiment I now refer to is the one purporting to illustrate and explain to advanced pupils the cause of the earth's equatorial protuberance. An elastic hoop is provided and so adjusted on a vertical axis that by means of a crank it can be rotated around its axis at a high velocity. As the crank is turned faster and faster, the hoop is seen to elongate horizontally and its vertical axis to shorten correspondingly, making an ellipse out of what was before a true circle. Here the experiment is invariably brought to an abrupt close. The lesson it is intended to teach is that the earth's oblateness is the physical effect of her axial rotation.

Now it is most earnestly to be hoped that no undergraduate can be discovered so preternaturally dense as to require to be shown this silly experiment in order to teach him no more than the a priori knowledge that such ENFORCED rotation must produce just such effects. What he wants to know, what you want to know, what the world wants to know, and what these blind teachers of the blind cannot answer is, What keeps the earth turning on her axis? The experiment of the hoop demonstrates admirably that as long as you are content to turn the crank the hoop will not only dutifully rotate, but that it will also remain distended. But it also most emphatically demonstrates that the hoop will infallibly cease doing either of these things the moment you suspend your exertions.

Suppose that, while the hoop is rotating at its highest speed and consequently expanded to the limit, you were sufficiently deft to insert in it a horizontal brace, you could then shut off the driving force without impairing the deformation attained; otherwise the natural elasticity 
of the steel will cause the hoop to spring back into its original shape. Again, you might accomplish this distension of the hoop in other ways, as, by setting a heavy weight upon its rim while it stood in an upright position, or by stretching it with your hands, or by compressing it in a vise. And yet again, if the hoop were very large, or relatively very thin, or composed of some softer material, as lead, its own weight would tend to flatten it. In short, there are more ways than one of deforming a hoop, and there is certainly a better way of accounting for the earth's oblateness than by calling upon the energy of her rotation; for which, both as to its origin and recuperation, Newtonians confess their inability to account. They resemble the thriftless wife who could not be made to comprehend why her credit at the bank had run out, when there was the check-book to prove she still had a plenty of blanks in reserve. Drafts on the Bank of Imagination are never returneed N. S. F., and Newtonians have certainly not been chary of availing themselves of its unlimited resources.

To ascertain just how much energy is required to distend our hoop to any given degree of elongation, all that is necesssary is to hang it on the hook of a balance scale, lend your weight to the hoop until the desired elongation is obtained, and then read the scale. To effect this elongation by cranking requires power-a steady flow of power or energy - exactly equal to the elastic strength of the steel, as previously ascertained on the scale, or, what is the same thing, to the sustaining against gravity of a weight of that same magnitude. It not only required a great expenditure of energy on the part of the earth's momentum to whirl the excess water to the equator in the first instance, but it takes a steady supply of power to keep it there.

Again. Suppose that the hoop instead of being made of spring-steel were made of some material that would not give while rotating, but continue to retain its original circular shape, what then? Which operation, think you, will require the greater expenditure of power; to rotate 
the rigid hoop at a given number of revolutions per minute, or, the elastic one? The deformation of the latter cannot, of course, be effected without the absorption of power; shall we then conclude that to rotate it at the same rate of speed as the other will require just this much additional power? Assuredly not! "But," you may say, "then the act of deformation does not absorb energy as at first supposed? How can we explain this apparent paradox?" The answer is plain enough. That part of the centrifugal force which in one case manifests itself in elongating the elastic hoop, that is to say, causes visible distortion, transforms itself into heat in the case of the rigid one by forcing its molecules violently against each other, from within outward: Let this pressure pass a certain mark and we have the phenomenon of an explosion, as, indeed, often occurs in the case of fly-wheels.

These reflections bring out still another radical distinction between my views and those of Newton, namely, that whereas he claims that, having once been given its rotatory motion, no matter how, a planet will continue to revolve forever from sheer inertia, I contend that the earth, even if it were absolutely rigid and possessed a mathematically spherical shape, would automatically bring itself to axial rest by converting and yielding up its centrifugal force into kinetic energy, or heat, and radiating the same out into space. There simply must be some driving power behind the earth's rotation. What is it? Is it natural or supernatural, mechanical or miraculous, real or imaginary, physical or verbal, inertial or creative? Calling a fly-wheel persistent will not enable us to dispense with the engine, nor will it serve any better for the earth's case.

The reader will doubtless remember that in estima1ing the energy of the earth's attraction upon the moon I adopted as a convenient, though perhaps somewhat arbitrary, standard one horse-power as equivalent to the sustentation of one ton, stationary, against gravity. This is obviously an overestimate, but as it is an error on the safe side, and as only approximate results will here be 
required, we may agree to ignore what moderate discrepancy there may exist.

We have seen how Newton, in his effort to make as plausible a showing as possible in the computation of his tidal heights, chose to construe the entire equatorial bulge, " 85,472 Paris feet" in thickness, as being peren-i nially centrifugalized from the polar regions; a mode of figuring in which our modern mathematicians servilely concur, albeit they have reduced this amount to some 70,000 feet. Let us take them now at their word and try to ascertain just what is meant by their allegation that this" enormous mass is being upheld, not by dams or any other natural bulwarks whatsoever, be it remembered, but by. sheer energy filched from the gratuitous; unrecuperated momentum of the earth's axial rotation. Of course, there is not as much water on, or in, the earth as these figures imply, but as our Newtonian friends reason on the basis of this assumption it is fair for us to follow their example. Allowing for the tapering of the thickness of this: aqueous sheet from the line of the equator to the poles; we derive as the total mass of water thus declared to be energially upheld $197,000,000$ square miles of area, 35,000 feet in depth. Computing the weight of this water on the basis of 63 pounds per cubic foot, redueing to short tons, and translating these into horse-power units under our improvised rule, we obtain the inconceivable quantity of, in round numbers, six quintillions of horsepower! Pray, dear reader, do not miss the point. Newton and his adherents do not contend that this feat of uplifting was performed ages ago, in one great, spasmodic, transient throe, or that natural barriers (mountain ranges, for example) intervened to prevent the fluid from immediately flowing back to the lower levels of the arctics, but that this great sea is continuously being whirled up from moment to moment, from century to century, and from age to age. If, pardonably, you should expérience difficulty in crediting our hard-headed men of science with being capable of standing sponsor for such preposterous opinions, let me ask you to ponder this 
explicit statement from no less an authority than Sir John Herschel (Outlines of Astronomy, Art. 224) :

Now, it is highly satisfactory to find that the general elliptical figure thus practically proved to exist, is precisely what ought theoretically to result from the rotation of the earth on its axis. For let us suppose the earth a sphere, at rest, of uniform materials throughout, and externally covered with an ocean of equal depth in every part. Under such circumstances it would obviously be in a state of equilibrium; and the water on its surface would have no tendency to run one way or the other. Suppose, now, a quantity of its materials were taken from the polar regions, and piled up all around the equator, so as to produce that difference of the polar and equatorial diameter of 26 miles which we know to exist. It is not less evident that a mountain ridge or equatorial continent, only, would be thus formed, down which the water would run into the excavated part at the poles. However solid matter might rest where it was placed, the liquid part, at least, would not remain there, any more than if it were thrown on the side of a hill. The consequence, therefore, would be the formation of two great polar seas, hemmed in all around by equatorial land. Now, this is by no means the case in nature. The ocean occupies indifferently all latitudes, with no more partiality to the polar than to the equatorial. Since, then, as we see, the water occupies an elevation above the center no less than I3 miles at the equator than at the poles and yet manifests no tendency to leave the former and run toward the latter, it is evident that it must be retained in that situation by some adequate power. No such power, however, would exist in the case we have supposed, which is therefore not conformable to nature. In other words, the spherical form is not the figure of equilibrium, and therefore the earth is either not at rest, or is so internally constituted as to attract the water to its equatorial regions, and retain it there. For the latter supposition there is no prima facie probability, nor any analogy to lead us to such an idea. The former is in accordance with all the phenomena of the apparent diurnal motion of the heavens; and, therefore, if it will furnish us with the power in question, we can have no hesitation in adopting it as the true one.

Now, everybody knows that when a weight is whirled round, it acquires thereby a tendency to recede from the centre of its motion; which is called the centrifugal force. A stone whirled round in a sling is a common illustration; but a better for our present purpose will be a pail of water, suspended by a cord and made to spin round, while the cord hangs perpendicularly. The surface of the water, instead of remaining horizontal, will become concave. The centrifugal force generates a tendency in all the 
water to leave the axis, and press towards the circumference; it is therefore, urged against the pail, and forced up its sides, till the excess of height, and consequent increase of pressure downwards, just counterbalance its centrifugal force, and a state of equilibrium is attained. The experiment is a very easy and instructive one, and is admirably calculated to show how the form of equilibrium accommodates itself to varying circumstances. If, for example, we allow the rotation to cease by degrees, as it becomes slower we shall see the concavity of the water regularly diminish; the elevated outward portion will descend, and the depressed centre rise, while all the time a perfectly smooth surface is maintained, till the rotation is exhausted, when the water resumes its horizontal state. (The italics are all his.)

Inasmuch, however, as I previously contended that Newton erred in not construing only a half of the equatorial protuberance as being centrifugalized, and in not allowing for the fact that the oceans are only a little more than two miles deep, let us generously accept even these low figures. According to the best authorities, the weight of the actual oceans is, in round numbers, 1,400 quadrillions of tons, of which, let us agree, only one-half is energially upheld by drafts upon the earth's axial momentum. Then it would appear, recourse being had to our rule, that the planet is using up only 700 quadrillions of horse-power, perennially, instead of the six quintillions that we found before. (Compare, also, Young, G. A., Art. 154.)

Again, accepting Sir Robert Ball as spokesman for his fellow astronomers to the effect that the energy that produces the tides emanates not from the sun or moon, but out of the earth's store of centrifugal momentum, let us see what this, also, amounts to. Flammarion gives the sum of the lunar and solar tides as (approximately) 30 inches at the equator. Taking their average height for the whole world as 15 inches, and limiting this again to only half the ocean surface (75,000,000 miles), gives a weight of water thus alleged to be perpetually whirled upward by the earth of, in round numbers, 80 trillions of tons. As this action is going on all the time, it means that, on this second count, the centrifugal force is wasting away at the steady gait of 80 trillions of horse-power. 
The complacent reader may think to himself that, huge as these drains upon the rotational energy of the earth seem to be, they must, nevertheless, be negligible in comparison with their source, else our Newtonian friends would not dare to draw upon that source so extravagantly as they do. Now, it is easily possible to ascertain the magnitude of this supply in terms of horsepower within reasonable limits of accuracy. Assuming that one-half of the earth's mass is contained within an inner core 6,000 miles in diameter, and that the average velocity of the whole mass is the same as the velocity of any point on the equator of that core, the distance covered in a single second would be 1160 feet. This is manifestly an overestimate, seeing that, in the higher latitudes, the velocity is necessarily much less, and, for convenience, we may arbitrarily reduce this quantity to $\mathbf{1 1 1 6}$ feet per second, which, though still too high, happens to be one-third of the orbital velocity of the moon and therefore simplifies the present calculation by furnishing us with a convenient standard for comparison.

Now in Chap. III, if you remember, I computed the energy value of the momentum of the moon, traveling as she does at the rate of 3350 feet per second, to be 2,112 trillions of horse-power days ( $8.8 \times 240$ trillions). Multiplying this quantity, then, by 81 because of the earth's greater mass, but dividing the product by 9 (the square of 3 , since the energy varies with the square of the velocity) because of her slower (axial) speed, we obtain the sum of 20,000 trillions as the total number of horse-power days "stored". in the earth's axial momentum at this given instant. Compare this solitary energy asset now, if you please, with our liabilities as previously appraised, and you will be startled to find that were it to be applied solely to the sustentation of the equatorial ring it would on the basis of Newton's version be 300 times too feeble to so much as even budge the weight, and on mine it would exhaust itself in the brief space of less than a single hour, or if applied wholly to the raising of the tides, in just 250 days! What is the answer? How comes it that our asset 
remains obviously intact in spite of these stupendous drains? Can Sir Robert be right in likening the earth to a fly-wheel detached from its engines? Did you ever see a "fly-wheel detached from its engine, but still connected with the machinery" fail to betray signs of slowing up almost immediately, and shortly come to a dead stop? Furthermore, what sort of an engine was it to which the terrestrial fly-wheel we call the earth was once attached, and how did it become detached, and where is it now?

Tide or no tide, equatorial ring or none, it is not true that a great ball like ours, even were it the only one in existence, could, once started, continue to rotate forever "because there would be nothing to stop it." There is such a something-gravity. It has been estimated that the earth would have to rotate 17 times as fast as it does before a loose rock, say, on its surface "would lose all its weight and fly off to become a self-sustaining satellite." Now, it seems quite plain that if the earth as a whole is to keep on turning itself round and round forever, every particle of it must be capable of doing its own carrying and not depend for levitation or propulsion on its neighbors, who have equal need of like help themselves. According to Newtonians, the rotation of the earth lightens any given object at the equator by only 1-289 of its weight, so that a mountain weighing, say, 289,000 tons may be provisionally construed to be self-sustaining by virtue of its "persistent" momentum to the extent of 1000 of its tons, but as to the remaining 288,000 tons it must be regarded as a burden imposed upon some carrying agency underneath it. The bed of rock on which the mountain reposes is, in turn, along with its load, a still weightier burden on the next stratum beneath, and so on down to the center of the earth. But the earth's center is only a mathematical point! Nowhere do we come across any sign of a motive power. There are only two ways of dealing with this mystery. One is by appealing to the miraculous, alias "celestial mechanies," as the Newtonians do; the other by placing the finger on the substantial cause. That cause, I hope to convince the reader is none other than the tides themselves. 


\section{VII}

\section{THE AUTHOR'S THEORY OF. THE TIDES}

$\mathrm{I}$

$\mathrm{N}$ this chapter I hope to convince the reader that tides are not created in the manner depicted by Newton, that is to say, in antagonism to the principle of equilibrium, but rigorously in accordance therewith.

In the diagram (Fig. 5) let $\mathrm{E}$ represent a planet endowed naturally with the property of gravitation. It consists, let us say, of a solid ball 10,000 miles in diameter surrounded by an envelope of water five miles in depth, and, as an aid to the imagination, suppose this water frozen to a depth of one mile. Let us now bring into touch with $\mathrm{E}$ a second body, $\mathrm{M}$, which has no seas and which, in the outset, possesses no gravitational power. Under these conditions E, of course, will not be in the least affected by M's proximity. But suppose we had a method whereby, as by the turning of a tap or switch, we could charge $M$ with as much of this force as we pleased, and let the current be then turned on. What would be the natural effect upon E? Surely its solid part would immediately begin to sink in its own seas and finally rest upon the inner surface of the ice crust (Fig. 6 ). In other words, the tidal action of $\mathrm{M}$ on $\mathrm{E}$ would be to depress the seas intervening between them and not to elevate them there at all. Now, if you please, turn on the discharging tap and see what will happen next; obviously the planet $\mathrm{E}$ will return to its original condition. 

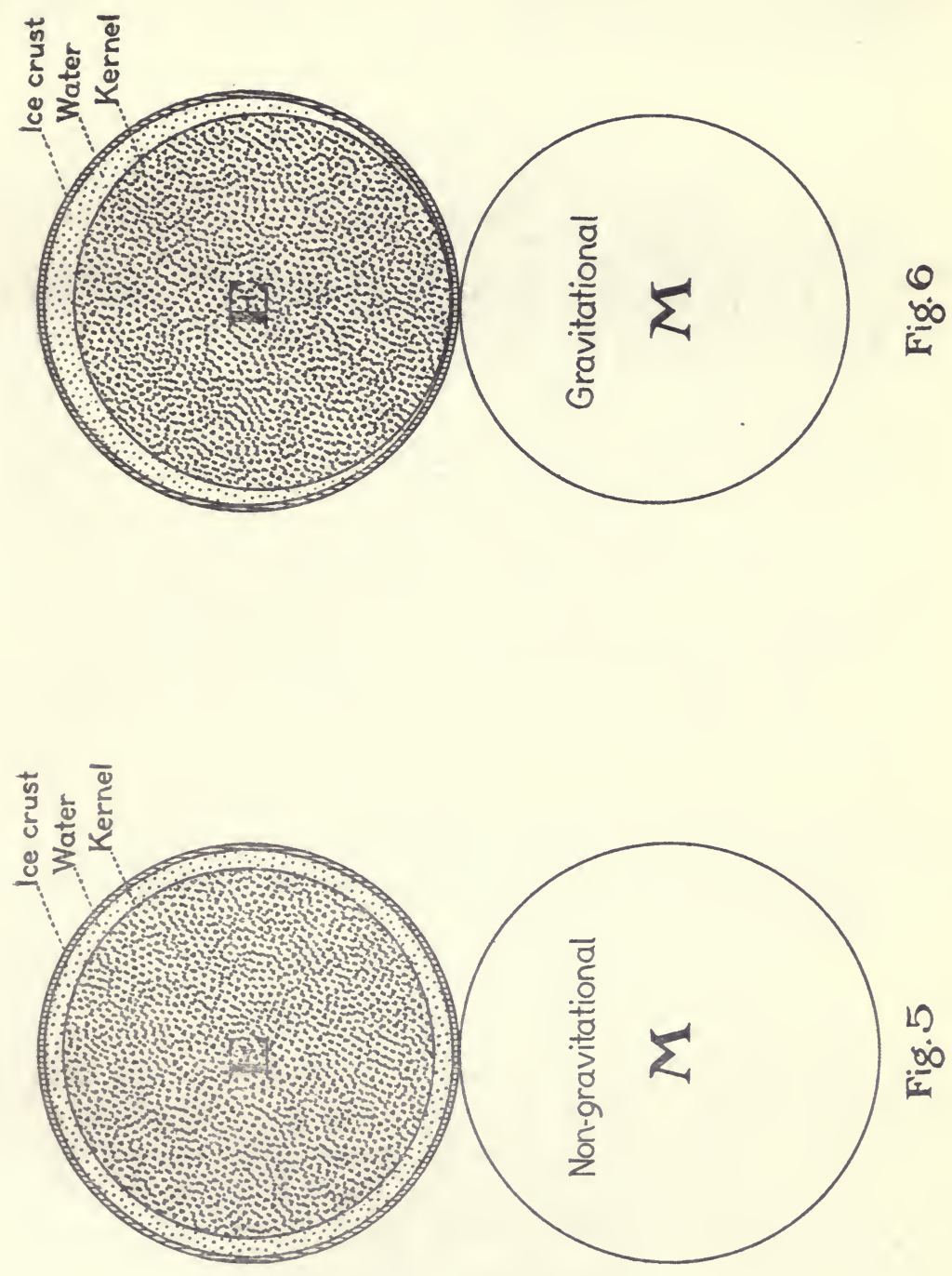
The same phenomena would have occurred, in like sequence, had we imagined the body $\mathrm{M}$ approaching $\mathrm{E}$ through a great distance. M might then have been postulated as gravitational from the start, the same as E, the element of distance taking the place of our artificial regulation of the gravitational intensity. Tides thus produced, by repelling (differentially) the water under the tide-causing body, would obviously conform to the phenomena, respecting whose "perversity" Darwin mistakenly complains.

Let us consider a second illustration: Imagine the universe blotted out save for a single cloud of aqueous vapor of, say, the same mass as the moon; then, under the principle of gravitation, the cloud would eventually condense into a watery sphere. Suppose, again, that instead of consisting of water vapor alone, it comprised equal parts of mercury and water; then there would result a planet containing an inner core of mercury, and, around it, a concentric sphere of the lighter material constituting a universal sea of uniform depth.

We will now conceive an exactly similar planet to spring suddenly into existence at a distance of ten million miles away and both planets to gravitate toward each other by virtue of their mutual attraction until they collide. Query: What would be the nature of their tidal deformations in transitu, assuming that they possessed no axial rotation?

To this question present-day astronomy vacillatingly returns two contradictory answers, consistent only in the respect that both are equally inimical to the principle of equilibrium; which, indeed, is precisely what they are meant to be. One of these answers is Newton's own, namely, that inasmuch as the power that causes the tides resides in the fact of rotation, there being in this case, by premiss, no rotation, there could be no deformation at all; hence the two spheres would arrive at their point of impact as perfectly spherical as when they began falling.

The alternative answer is that of the hypothesis of statical tides, a notion which astronomers adopt without 
hesitancy whenever it suits their purpose, in spite of its gross logical inconsistency. This view asserts, that the spheres in question would elongate in the direction of the line of descent, so that by the time they reached the point of collision they would somewhat resemble row-boats meeting bow on, both their pyramidal prows and sterns consisting wholly of water, while the heavier element, mercury, would comprise the middle section.

In order to expose the fallacy of both these views, let us try the time-honored plan of picking up the other end of the skein, and working backwards. Suppose, then, the collision to be a thing of the past and the resultant body to have had time to compose itself, as it naturally would, into a new sphere in all respects like our two original ones, only, of course, twice their size. Conceive this major sphere to be cleanly severed in half, and the divided parts to be gently removed from each other to their original separating distance. Without doubt, by the time they arrive at those extreme points, the two hemispheres will no longer be such in shape, but will necessarily have acquired their aforetime sphericity. The question then arises as to what should be the intermediate or transitional shapes.

May it go without argument, that the chain of transformations, going and coming, should be the same, only in reverse order? If this be conceded, then, according to Newton's idea, the hemispheres immediately following their sundering should instantly leap into spheres then and there, without waiting for further removal. On the other hand, according to the Statics, they should, instead, but quite as spasmodically, reassume their boat-like forms, and from then to the end of the outward journey gradually exchange these for the spherical.

According to my theory of tides by equilibration, on the contrary, each of the two small spheres, in the course of their mutual approach, would undergo very gradual modification of shape through forms less and less gibbous, imitating the phases of the moon between full and last quarter; while the hemispheres, on the other 
hand, in the course of their separation and removal to the original distance, would naturally repeat these phases, but in just the reverse order (See Fig. 7).

Speaking specifically of the earth, then (though the principle applies universally), her tides are caused by a conflict between two ruling gravitational forces, namely, (1) the integral attraction of the earth upon her own mass, and (2) the extraneous attraction of the sun; the first seeking ever to concentrate her heavier substances at her center and to deploy the lighter on the outside, the latter ever striving to compel her to recognize the sun's rival power and advance her heavier parts toward him. Were the earth solid clear through, as the moon appears to be, she would turn the same face to the sun continuously, but having great mobile oceans, she compromises by shifting them from in front of the sun, thus giving her center of gravity, as it were, a chance to make obeisance to him.

\section{Computation of the Tidal Forces}

It will be remembered that, according to Newton's idea, the waters making up the tidal mounds are construed as being drawn thither obliquely from the rounded sides of the earth; whence he argues that the tidal forces of the sun and moon do not vary in accordance with the rule of inverse squares, as the law of gravitation has it, but of the inverse cubes. As a result of this hypothesis, he estimated the ratio of the sun's effect to thar of the moon as (about) one to four. If we are right, however, in our new hypothesis that the tides are acts of equilibrism, the solar and lunar tidal forces vary, not according to the exception, but strictly according to the letter of the law itself. Thus calculated, they vary as 180 to 1 , this time with the sun having the advantage-a total change of 720 points!

Knowing as we do the relative masses of the earth and sun (1 to 332,000) and the earth's radius as compared with the solar distance ( 1 to 23,000 ), we can easily find by applying the law of gravitation that the earth's at- 


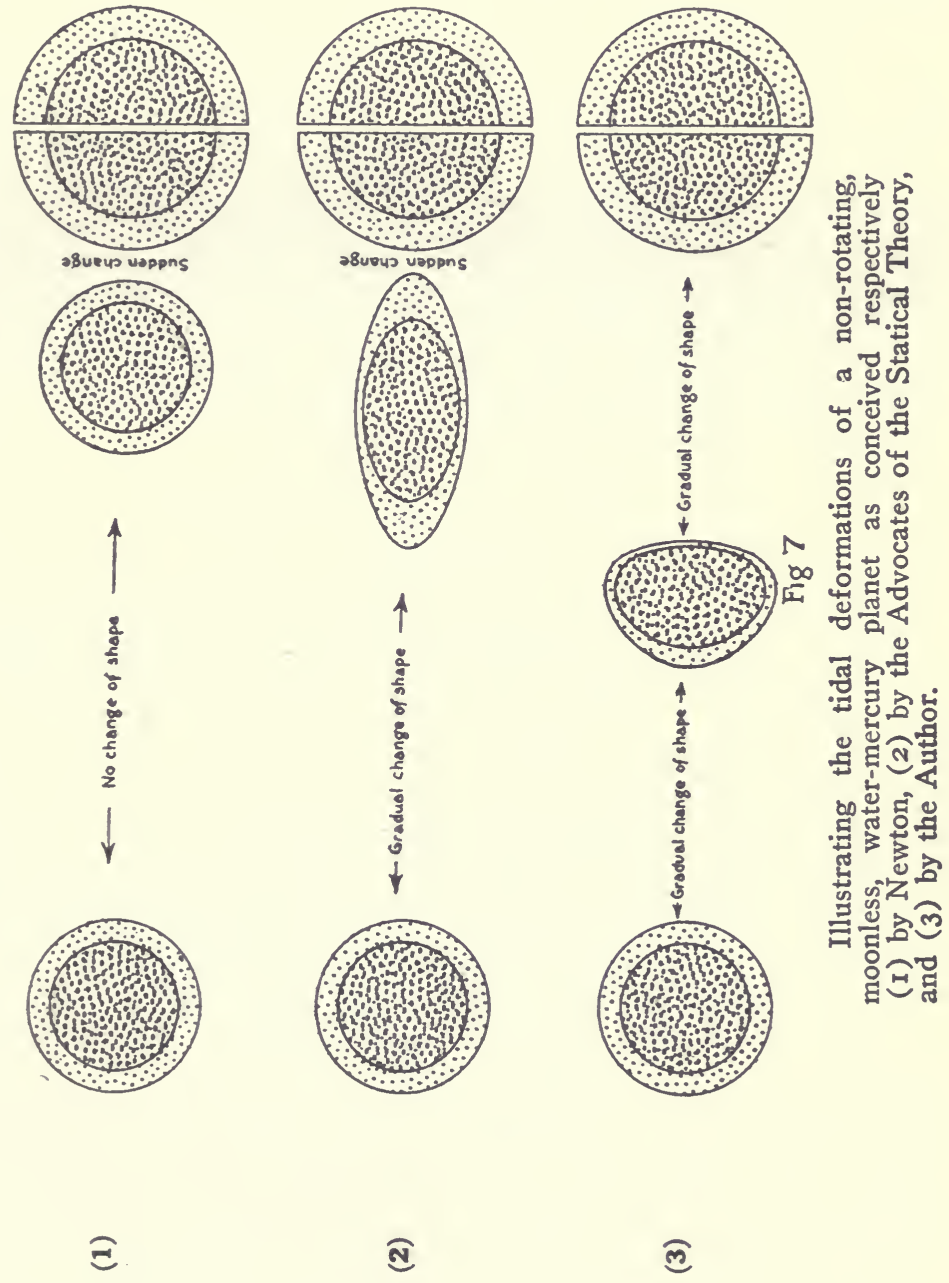


traction on her oceans is, in round numbers, 1600 times as powerful as the sun's. Here, then, is the measure of the conflict of authority between the earth's integral attraction on the one hand, and the sun's disturbing attraction on the other; the former commanding the oceans to remain where they are, the latter ordering them to give way and let the earth's center of gravity step forth to greet him. In order, now, to ascertain the tidal force of the sun we have only to divide the average depth of 12,480 feet by 1,600 , yielding 7.8 feet; and by dividing this quantity in turn by 180, we find the moon's effect to be barely half an inch. That is to say, the tidal action of the two bodies tends to depress the level of the ocean directly under them by the amounts stated; the waters thus displaced causing flood tides elsewhere.

\section{The Cause of the Earth's Diurnal ROTATION}

It has been previously demonstrated, not only by the author's original arguments, but by the quoted admissions of such orthodox authorities as Sir Robert Ball and Sir John Herschel, that Newton's premises presuppose a miraculous origin of the earth's axial rotation and, what is more, provide no recuperating source to make good current losses. As if this were not taxing human credulity enough, we have been further taught: first, by Newton and Herschel, that the equatorial ring is being centrifugalized throughout the ages by this mysterious power; second, by Kant, that the rotation is being slowly destroyed by the friction of the tides; thirdly, by Ball and the rest, that all the energy that goes into the lifting of the tides flows from this same store of unearned momentum; and, lastly, that, marvel of marvels, the historical records of some five thousand years of eclipses inform us that, in spite of all these monstrous drains, the earth's day has not lengthened by so much as a hundredth of a second of time! Truly, celestial mechanics as taught by our "great scientific institutions" is quite as far above the heads of common mortals as Dr. Abbot says. 
Counting myself as one of this plebeian caste, I confess, without any sense of shame, my simplicity in supposing that the earth's axial rotation had a dynamical origin, that it is being spun round and round by a live power, and that the much taking of something from a finite quantity, though possible to the hypercultivated imaginations of our Newtonian friends, is neither good mathematics nor good sense.

That some physical cause lies behind the earth's rotation is axiomatic; and what cause more eligible to this purpose than this very phenomenon of the tides, superinduced as they are by that great cosmic force-gravitation? Where can power be more effectively applied than at the circumference of the fly wheel? Has it never occurred to you before, that Newtonianism has found no use for gravitation save as a restraining means-to leash planets and retard rotations? Given this unique, this all-sufficient creative energy, modern science has systematically resisted its logical development. Like the unworthy servant in the parable, it has buried its talent.

Here we have the precise elements requisite to account for the earth's diurnal rotation. In Fig. 8, let $A B$ represent an arc of the earth's orbit, along which she is being hurried by the vortical action of the Prime Resultant, and E, our planet itself (the direction of the movement in each case being shown by the arrows). As previously explained, the tidal action of the sun at the point indicated by $\mathrm{x}$ is to drive back the oceans with a strength equivalent to 1-1600 of their weight; it is as if the sun were applying a very powerful brake, or as if he were blowing a strong blast against that side, causing the solid kernel to trip, as it were, and roll over and over upon its face, like a barrel on an incline. Or, as some might say, the principle is rather that of buoyancy, the kernel being "lifted off its feet", as by a sudden rise of water, rendering it perennially unstable against the pull of the Prime Resultant. A stately ship, our Earth, driven by wireless power from the stars; herself the compass, humanity her passengers, eternity her port! 

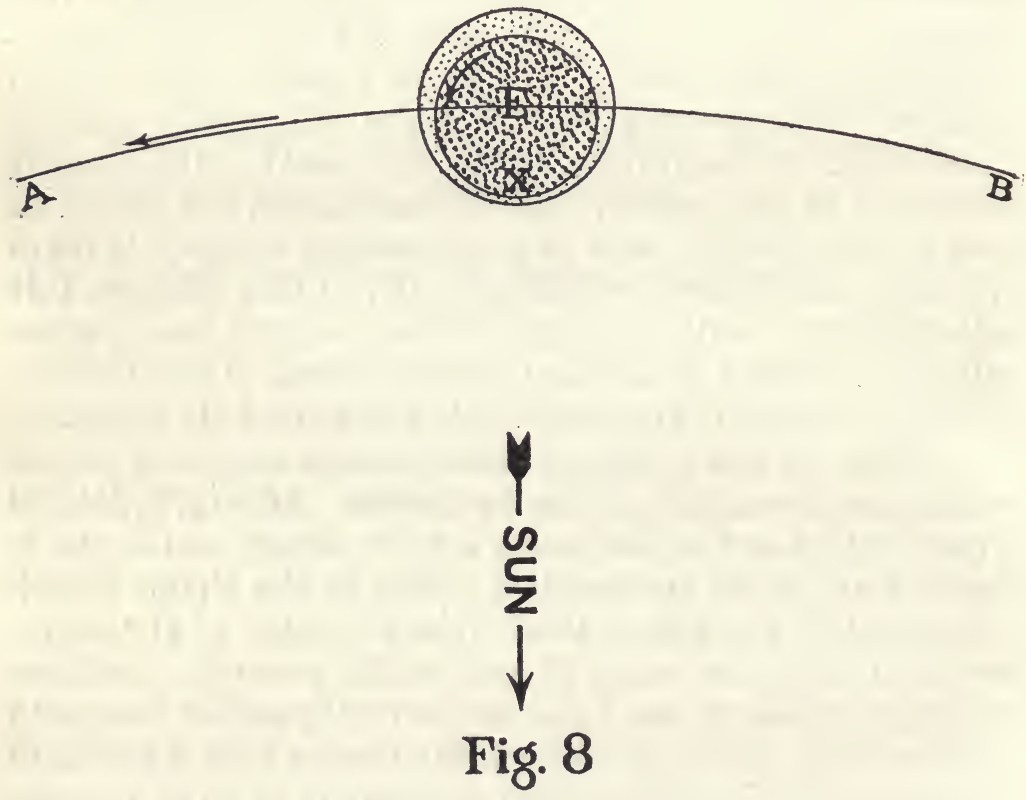
If we care to, we can imagine a beginning to this pro. cess from a previous state of rest, and picture to ourselves a gradual acceleration of the movement until, like a fly-wheel responding to its engine, the planet attained the uniform velocity it now seems to possess. However, the applied forces are not absolutely uniform the year through, for not only is the earth nearer the sun at perihelion than elsewhere in her orbit, but that place happens also to be the position of the winter solstice, when a greater ocean expanse is exposed to the sun's tidal influence; besides, the earth travels faster in her orbit then than at other times. For these reasons, the spring days (24 hours) must be a few seconds shorter than those of autumn, but whether our chronometers are reliable enough to register these nuances is doubtful. Before the time of Kepler the world mistakenly supposed the planets to revolve at uniform speed. Why, then, may not also axial velocities periodically vary? Such variations might easily exist without incurring observational discovery, inasmuch as they would not necessarily affect either the length of the average day or of the year.

Lifting our eyes to other planets, the first they behold is Mars, whose mass is only 1-9 that of our earth. Situated as he is about half again as far as we from the sun, the latter's attraction upon him is less than half as great as it is on the earth. Now, Mars, too, rotates on his axis, and his day is only slightly longer than our own, all of which harmonizes admirably with our hypothesis of tidal rotation. There can ultimately remain only one conclusion, namely, that Mars is richly endowed with oceans, proportionately more expansive, though shallower than ours. When the first edition of this work appeared in March, 1912, it was difficult to discover in the books any testimony in support of such a deduction. In fact, the reports were almost if not wholly the other way, making the planet out to be a veritable desert. During the last two oppositions, however, overwhelming evidence of a contrary nature has grown apace, some of which will be adduced in a later chapter. As for the 
axial rotations of the sun and Jupiter, with their pecul. iar problem of equatorial acceleration, and the rotations of the other planets in general, these must be postponed to a later chapter.

\section{The Turning Force Computed}

Before leaving the subject, it may be well to ascertain the degree of this buoyancy, or, if you prefer, the pressure of this brake.

The sun illumines only one-half of our planet at a time, and on that half he does not shine all over vertically, but mostly obliquely. Accordingly, in estimating his tidal action we may average it up as affecting only one-fourth of the ocean area at one time, but this much with maximum effect. This area is given by Murray at $140,000,000$ square miles, of which the fourth part is $35,000,000$ square miles. A sheet of sea water (63 pounds to the cubic foot) 7.8 feet in depth (the solar "tide"), extending over this immense expanse would weigh 240,000,000 million tons, an amount significantly identical with my former estimate of the intensity of the earth's attraction upon the moon. Converting this into horse-power, according to our chosen formula, it becomes quite evident that the earth is not turning on its axis simply "because it cannot stop a motion that never was started", but because Nature intelligently supplies a quarter-quadrillion-horse-power engine to do the work. This, too, in addition to the coopperation of the Prime Resultant, pulling the earth along in her spiral orbit.

\section{The Moon's Relation to the Tides}

Although Newton estimated the ratio of the solar and lunar tides as 1 to 4 , modern computation places it at 1 to 2.25. Were either of these ratios true, it is hard to understand why no trace of a solar tide has ever been detected. To say that the lesser merges in the greater, even though the circumstances for their separate existence are at times most favorable, is only a subterfuge. 
The absence of such a double set of tides is, however, easily understood under my theory, for the lunar tide is reckoned at only 1-180 of the other relatively, and only a half-inch absolutely-far too insignificant to show.

A more important problem, however, is : Why do the passage of the moon and the appearance of the tide, even though the latter is some eight hours in her wake, ex. hibit such a singular correspondence in their schedules?

The whole mystery dissolves away with the discov. ery of the true tidal cause. Without being able to fathom the physical reason, astronomers have discovered that changes of momentum in this or that member of a cosmic system, whether orbital or axial, are invariably compensated by reverse changes elsewhere. They call this "the law of conservation of moment of momentum". With them it is only an empirical fact, an arbitrary provision of Nature's, dictated neither by discoverable reason or necessity.

Our present inquiry as to why the moon and the tidal crest keep pace, affords us an excellent opportunity to emphasize and illustrate the advantage of the principle of equilibrium over the denial of it as a key to the structure of the cosmos, and to point out the superiority of physical causation over cryptic abstractions. The studious reader who has done me the honor to read thus far must now be as familiar as myself with the concep. tion of what a "gravitational unit" is, and how our binary earth-moon system maintains its balance, progressively, in the face of all adverse outside attractions. One-the chief-disturber of this equilibrium is, of course, the sun. By virtue of his differential attraction upon the kernel of the earth and her seas, the planet is obliged to redispose her mobile parts-to wit, her oceans -in such a way as not only to trim her own balance, but that of the little system of which she is the main cog. The question is, whither shall the waters squeezed out from under the sun go, so as restore the system's shaken balance? Certainly not to the spot under the moon, for that would only be making matters worse by still more 
overweighting that already overweighted side! No, the natural way is for the migrating waters to heap themselves up at two points on the surface equi-distant from the moon and from each other, forming together, as it were, the three corners of an isosceles triangle. These locations, of course, are ideal rather than actual, inasmuch as the moon, because of her monthly revolution, is constantly changing her angular position relatively to the earth and the sun, thus dynamically complicating the phenomena themselves, as well as the mathematics of them, to an extreme degree.

It is here very important to guard against the mistake of supposing that the sun's displacing power upon the waters is necessarily always uniform. Were this true in fact, it would but cloud the principle involved. The principle is that the earth must maintain her own and her system's equilibrium, and so she reacts to the attraction only to the extent required by the conditions of the moment. For instance, when the sun is mid-over either ocean, his effect is at a maximum, and when he is looking down upon the continents, at a minimum; likewise, in winter, when he is farthest south, where the oceans are twice as expansive, the tides are in general higher than when he is north of the equator. The position of the moon in her orbit is another factor that rules the heights of the tides. When she is in line with the earth and sun, the system is top-heavy, and needs more ballasting than when she is elsewhere, hence it is then that we have our "spring" tides; but when she is at quadrature, the contrary is the case, and we have "neap" tides; and so on.

This principle of what may be called tidal equilibrism furnishes the sufficient explanation of the singular movements of the inner satellites of Jupiter and of Saturn, described by Sir John Herschel in Articles 542 and 550, respectively (Outlines of Astronomy):

An extremely singular relation subsists between the mean angular velocities or mean motions of the three first satellites of Jupiter. If the mean angular velocity of the first be added to twice that of the third, the sum will equal three times that of the 
second. From this relation it follows that if from the mean longitude of the first added to twice that of the third, be subducted three times that of the second, the remainder will always be the same or constant, and observation informs us that this constant is $180^{\circ}$, or two right angles; so that the situation of any two of them being given, that of the third may be found. *** One curious consequence is, that these three satellites cannot be all eclipsed at once; for, in consequence of the last mentioned relation, when the second and third lie in the same direction from the centre, the first must lie on the opposite. $* * *$

A remarkable relation subsists between the periodical times of the two interior satellites of Saturn and those of the two next in order of distance; viz., that the period of the third (Tethys) is double that of the first (Mimas), and that of the fourth (Dione) double that of the second (Enceladus). The coincidence is exact in either case to about I-800 part of the larger period.

That the atmosphere should be affected by tides in much the same manner as the hydrosphere goes without saying, but on account of its invisibility, its tidal behavior cannot be so exactly investigated and defined. One point of this relationship is revealed by the observed fact that when the ocean level is low, the barometer is always higher than normal. It is atmospheric tides that constitute the chief cause of trade winds, but I shall not weary the reader by discussing this collateral subject in the present work.

\section{Terrestrial Magnetism}

By substituting, in theory, tides of depression for lifting tides, we not only gain in frictional effect, but we likewise transpose the locus of it to the floors of the oceans instead of to their coasts. On the other hand, I have given reasons to justify the deduction that the metals, being the heaviest substances, are mainly centrally located within the earth, and ranged along its axis. The semblance of the earth, therefore, to a helix and core, taken in connection with the dynamical effect of the tides, leads easily and naturally to the conclusion that the earth's magnetism is tide-induced. If added proof be demanded, it is to be found in the remarkable coincidence of the magnetic needle's variations with 
those of the diurnal movements of the sun and moon, and, quite as remarkable, with the change of seasons as well. Indeed, on careful investigation it will be found that whenever the water is severely shaken in the ocean bowl, by tides or by any other cause (solar explosions, for instance, which temporarily unbalance the earth as well as all other bodies in the system), extraordinary electrical phenomena are certain to follow. Heretofore, attempts have been made to connect the needle's variations with the fluctuations of the solar radiation, but unsuccessfully, because of the conflicting fact that the moon, whose radiation is comparatively nil, has been found to exercise a similar magnetic influence rivaling the sun's own. Orthodox astronomy has not dared to broach this tidal explanation, because it would involve too brazen a drain on inertial momentum; but the explanation becomes fully available under my own theory of gravitational centrifugal motions.

According to this logic, the earth's poles, being the termini of the supposed "core", should be the centers of greatest electrical activity, the charge passing from one or the other of them through the atmosphere, along meridianal lines, to the opposite pole, thereby completing the circuit. This surmise accounts at once, and equally well, both for the northern lights, or aurora borealis, and for the direction and deflection of the magnetic needle, besides offering a most alluring field for speculation as to the possible future exploitation of another natural resource.

\section{The Comets}

Beautiful though some of them are, the comets have ever been regarded by the ignorant as forerunners of disaster, and as disasters have a way of occurring right along, the comets have not failed to sustain their unenviable repute. Scientifically, they are generally looked upon as strays from neighboring systems, and this character they undoubtedly possess, notwithstanding that many of them have succeeded in insinuating themselves 
as permanent occupants of our solar hearth. Their manners it is that proclaim them strangers; they move eccentrically, redden when they near the sun, and presently turn tail and hurry away, perhaps never to return. But what, in truth, are they, and why do they behave as they do?

Imagine two bodies of equal mass away off in space and separated from each other by a considerable interval, and, midway between them, a cube of lead and a similar cube of ivory glued firmly face to face. Under these circumstances, clearly the bi-cube would take up a neutral position, disposing its longer axis at right angles to the line joining the attracting bodies. Suppose, now, one of the planets to remain stationary while the other is violently projected straight toward it. In this case, according to my interpretation, the bi-cube will incline its lead end toward the nearing planet and, by the same token, turn its ivory end toward the stationary one, more and more until, finally, the long axis, instead of remaining transverse, will run parallel with the line of force. An unreflecting person, watching the whole proceeding, might misinterpret this action of the double cube as attraction upon the lead and repulsion on the ivory; but we know better than that, because we know that ivory by itself will fall as well as lead, so it too must be attracted. Indeed, had we mated the ivory cube with a cube of cork instead of lead, it would have enacted the very same role as the latter. Stated generally, differential attraction often creates the illusion of repulsion.

Let us carry the experiment a step farther and imagine, in lieu of the bi-cube, a sealed tube many miles in length, nearly filled with equal volumes of a dozen mutually insoluble liquids, like mercury, water and benzol, and repeat the first process in the same order. Can you doubt for a moment that the tube, like the bi-cube, will at first orientate itself transversely to the course of the equal attractions, and, afterward, as the projected body draws nearer and nearer, will incline its mercury end more and more toward that body and its benzol end cor- 
respondingly toward the distant stationary one? And when, finally, the moving body comes close up, while the other continues still as distant as ever, don't you know, as well as you can reason yourself to any knowledge, that the tube will stand altogether erect, as did the soldered cubes?

But our picture is not yet complete, for if we do not speedily find some way to support the tube against the major attraction, there will presently be a collision and the contents of our vessel will be ingloriously spilled. For this sustaining purpose, let us invoke the vortical suction of the Prime Resultant, and, incidentally, let the sun's powerful attraction supply the place of the proximate body. Then we shall have the odd spectacle of a great long rod circling the sun-veritably a visualized vector. Alas for our oddly constructed planet! In nearing perihelion, the sun's heat has proved too much for it; the mercury, indeed, has weathered the test, but the water and benzol have evaporated and, expanding, burst the tube and behold-a comet!

In the next chapter I' shall explain how the real comets are formed by the explosion of neighboring stars spraying their molten materials in all directions, some of them toward us. One such big spray, being hurled with great force, quickly finds itself in a region of intense cold, where its globules soon congeal, sealing within their chilled exteriors all sorts of stellar gases cooled down to inaction. Continuing onward in our direction, at high velocity, the comet, once fairly caught in our systemal vortex, acquires an orbit, necessarily greatly elongated, but varying in inclination and direction of motion according to the angle at which it made its entry.

During transit from its old home to its new, a journey which consumes literally thousands of years, the comet (because its motion is one of explosive and not gravitational origin) soon finds itself so far away from strong rival attractions that it is able to make its integral attraction among its scattered parts dominantly felt, and, eventually, though its aggregate mass be slight, to con- 
solidate them into a single ball, or perhaps into a vortical swirl, or gravitational unit, of small diameter. After entering the sphere of the sun's influence and connecting up with its predestined orbit, as it were, the comet forges on in regular course to its perihelion. Had it now nothing but its projectile momentum to depend upon, it would lose so much of this in "turning the corner" that it would inevitably fall in upon the sun. But, thanks to the torsional force of the quiver of rays of attraction that make up the Prime Resultant, the comet is upheld against the straining sun and whisked past his eager grasp, thwarting that Tantalus of his expected feast.

The crisis, however, has not been without its serious effects. The fierce heat from the luminary falling upon the massed particles, though perhaps not intense enough to fuse their crusts, is certainly able to raise their general temperature to a high state, thereby causing expansion of the confined gases, and so giving rise to multitudinous explosions of every degree of intensity. These phenomena, in the very nature of things, are most pronounced when a comet is very near the sun; which is unfortunate for human observation, because of the blinding contrast of the solar rays. The effects, however, are sometimes sufficiently pronounced to be seen and studied even despite this handicap, and reveal themselves in such strange phenomena as glowing nuclei, jets, envelopes, coma, and tails.

\section{Cometary Phenomena Explatned}

In nearing the sun, the integral attraction of the comet becomes dwarfed in competition with the rapidly augmenting solar attraction and a new disposition of its component substances is rendered imperative. Instead of preserving its old sphericity, therefore, it tends to the columnar form, with its heavier elements at the base (sunward) constituting the nucleus, and its lighter elements, mounting rearward one upon the other in the order of their levity, making up the tail. Newtonians, consumed as they are with their unreasoning antipathy 
to the principle of equilibrium, argue that the tail ought to point toward the sun, his attraction upon it being superior to that of the nucleus; just as though the latter were something nailed to the sky and had itself no affinity, not to mention superior affinity, for the sun. In. nature, comets exhibit tails only for a few weeks, or at most months, before and after their perihelion passage, and invariably point them away from the sun. Sometimes these tails are upwards of a hundred million miles in length, and in order for the tip to remain behind the nucleus, the former has been known to cover a distance. of 40,000 miles in a single second!

Imagine such a comet suddenly clapped into a giant test-tube and the tube gently lowered to the sun; where would you expect to find the nucleus? Surely not in the top of the tube! And the lighter gases, would you look for them to precipitate to the bottom? Of course not! Conceive the tube with its contents translated back again to its old place in the heavens and the container annihilated, can you conjure up any good gravitational reason why the tail should now alter its poise? No! Cometary tails, far from contradicting the principle of gravitation, positively illustrate and confirm it, as do they likewise vindicate the universality of the natural law of equilibrium.

Yet such is the singular perversity of Newtonian: philosophy that it has actually gone to work and invented a new cosmic "force" to counteract gravity in order to bring about the very thing gravitation implies! Astronomers call this chimerical thing "light repulsion", and, to eke out its already developed shortcomings, they have tacked on to it an even more ethereal idea, which they call "electric repulsion". They tell us, with Pickwickian seriousness, that the sun's light exerts a pressure upon the infinitesimal motes of cometic matter, not only strong enough to counteract the solar gravity, but also to carry the particles outward with the velocity of light itself. Expanding this idea, they go on and picture to a wondering laity how come- 
tary tails are shorn away in the very act of growing on, and, waxing still more eloquent, how they are carried away on the caudaliferous ether to distant bournes where, foregathering with other such ghostly moultings, they hatch out new planetary systems. Several mathematicians have gained esoteric fame by calculating this and that about the size of these motes and defining the relative intensity of light and electric pressures as contrasted with gravity, etc., etc., so that the theory may now be said to have as respectable standing among Newtonians as the old standbys of uncaused motions, irreducible momenta, and the rest.

On the occasion of its initial visit to the sun the comet is naturally charged with a greater cargo of gases than at any subsequent apparition; hence, after a few returns, it ceases to be visible to the naked eye and then becomes telescopic only, or is lost altogether to human sight. As long as the comet continues rich in occluded gases (whether the visit he its first or not) and its perihelion distance is sufficiently short, its advent is accompanied by an interesting display of celestial pyrotechnics. After playing its harlequin tricks for a gay month or two and casting its fiery confetti in the eyes of the staid old planets, it returns on its long road through the dark to its home in aphelion. As it so retires, it collects its scattered elements-its integral attraction more and more reasserting itself as the sun is left behind-until, after no long while, it rolls itself up into a ball as self-containedly as the earth herself. With each return, the aging comet becomes less flamboyant, its orbital eccentricity less pronounced, its inclination less arbitrary; in fine, it grows domesticated and turns into an asteroid. It is not true, however, that all asteroids have once been comets or, conversely, that all comets become in time asteroids; for many of the asteroids are native to our system, the same as the planets, while the comets may graduate into satellites, or, indeed, may end their careers by falling in upon the sun or one of the planets. This much, nevertheless, can be asserted without qualification, 
namely, that those asteroids whose eccentricity of orbit is strongly marked are of cometary origin.

Comets have their little brothers-called meteors. This is only to be expected from the very nature of the case. It would be extraordinary indeed if by the explosion of a star only one fragment, or one congeries of fragments, should reach us; rather should we expect a long train of scattered débris, many millions of miles in length, composed of congealed globules of all sorts of miscellaneous sizes. Thus Comet I, of 1861, has been identified with one stream of meteors, Biela's comet with another, and so on. Not only this, but there is a great deal of spectroscopic evidence that comets possess a chemical constitution strikingly similar to that of meteorites, which latter, of course, are only meteors that have managed to reach the earth. All of these objects, jssuing in a fused state from the shattered hulk of their parent star, and consequently more or less finely divided, cool quickly in the frigid realms of space, and so remain until their projectile motion is suddenly arrested, or they come near the fiery furnace of the sun. When either of these events occurs, the meteor explodes because of the sudden expansion of its freshly heated gases within. The reason why only the larger meteors attain the earth in safety is because the friction of our atmosphere is insufficient, by reason of its shallowness and lack of greater density, to do more than slightly fuse their outside, as actual cases have proven. Sizable meteors that do explode are known as bolides.

Here I fancy some of my readers exclaiming: "You are very inconsistent. Once you argue that flatness is the best condition for stability, again, that the gibbous and hemispherical shapes are, and now you champion the columnar form-how can you possibly reconcile these contradictory statements?" My defense is, that if there be any inconsistency, the fault is Nature's, and not mine. No one, for example, will think of questioning the anomalous phenomena of the gyroscope, or that a heavilytipped pestle, though laid flat on the table, will spring into 
an erect position, or that oil, though possessed of weight, will float on water, or that an inflated balloon will rise away from the earth that attracts it, or that a ball of dough will flatten into a thin layer. The circumstances in each case control, and our judgment must guide us in making our interpretations. Before leaving this subject, however, I deem it proper to forestall one possible misconception. I do not wish to be understood as implying that any representative fractional part of the mercurywater sphere would possess a hemispherical shape at the moment of collision. On the contrary, each such fractional part at the crisis mentioned should be similar in form to a symmetrical part of like mass already incorporated in the equilibrated unit.

In holding that comets are intruders into our solar system I am knowingly opposing the latest teaching of the astronomical fraternity. They used to tell us, only a few years ago, that some comets were indigenous and others not, basing conclusions on whether their respective orbits were elliptic or hyperbolic. Now they tell us that all of them have their origins within the system, because, forsooth, later mathematical investigations have shown that the hyperbolic curve may be produced by the perturbations of the planets.

But mathematics is only one phase of this manysided problem. It lacks correlating virtue. It throws no light whatsoever on how comets come into existence, on their relative ages, on their chemical constitutions, on how they acquired their translatory motions, on why their orbits are so elongated as compared with those of the planets, on the reasons for their usually high inclinations, on why some are retrograde and others direct, on their relation to the asteroids, etc.

\section{Remarkable Comets}

In order to appreciate the interpretive value of the principles just elucidated, the reader should compare the descriptions of past comets given in such books as Flammarion's Popular Astronomy. Take, for example, the 
great comet of 1843, which passed its perihelion within 33,000 miles (less than 1-25 of the sun's diameter) of the solar surface, at a velocity of 342 miles a second, through a hot blast capable of vaporizing steel, and in the teeth of a coronal resistance of undoubted immensity-yet came through the ordeal apparently unscathed, and without any perceptible retardation. More than this, it brandished a tail 198 million miles in length! 'The tail kept well behind the comet, consequently the tip of it must have swept through space at the velocity of about 40,000 miles in one second. Supposing that the tail averaged this length for as much as a single week, then, according to the hypothesis of light repulsion, the comet must have simultaneously grown and lost some 700 of these appendages!

Of Encke's comet we are told, first, that its period is diminishing, second, that it is not doing so as fast as formerly, and, third, that its head gradually contracts as it nears the sun and expands again as it recedes. Astronomers have been able to suggest only one way to explain the first two phenomena, namely, by postulating the ether as slightly resistant_- "not enough to hurt, yet just enough to serve". Newton, you remember, was scrupulously particular to have it understood that the ether is absolutely imponderable, knowing as he did full well that even the least possible retardation of the planets would inevitably destroy the "divinely-ordained"' equality of the centripetal and centrifugal forces and bring the whole system to grief. But even if we are gracious enough to wink at this modern indiscretion, or to concede the paradox of a medium by its resistance causing a hastening of the comet, as these ingenious savants declare, we cannot decently go further and admit that the degree of that resistance is declining in the same galloping ratio as required by the premises.

The mystery is easily cleared when it is recognized that Encke's comet is a comparatively recent intruder into the solar system; as is indicated by the fact of its having the highest eccentricity among the short-period 
comets. This, taken in connection with the additional fact that it is the innermost of its kind (where the vortical force is strongest), renders the disciplining process, at its present stage, especially noticeable and rapid. In short, its peculiarities of motion are owing to the gradual rounding of its orbit, the effects naturally dwindling as the process of approximation to perfect balance progresses. As for the contraction of the comet's head on approaching perihelion, this is obviously due to nothing else than the columnifying of the fused cometary nucleus by the sun's differential attraction-a phenomenon of equilibrism pure and simple.

\section{Latitude Variations}

About the year 1890 astronomers began the investigation of a unique phenomenon. This consists in the circumstance that the latitudes of every place on the earth's surface vary slightly from day to day the year through, though they always return periodically to what may be termed their home parallel-much as the sun crosses and recrosses the equator. In more graphic language, the earth seems to wabble on its axis as though that were a material axle and had worn itself thin in its bearing, giving room for play. To illustrate: Let A be a point on a given parallel and $B$ another point on the same parallel, but $180^{\circ}$ distant in longitude; then when observation shows the latitude of A to be slightly above the parallel on which normally it belongs, the latitude of $\mathrm{B}$ on that same day will be found just the same distance below, and vice versa. To explain this phenomenon a dozen hypotheses of various sorts have been advanced, but, so far, none has attained the stage of demonstration. However, all astronomers are a unit in believing that the phenomenon can be due only to an actual shifting of the earth's axis with reference to the crust. In this view I, for one, do not concur.

Doctor S. C. Chandler, the eminent American astronomer, has ably and carefully analyzed the observa- 
tional data and found that this movement of the pole can be expressed by a formula containing two terms, one of which varies between $85-1000$ and 185-1000 of a second of are, covering a period of about 430 days, and the other between 115-1000 and 155-1000 sec., whose fluctuating cycle is one year. These displacements, in terms of feet, are respectively between 9 and 20, and 12 and 15 (a second of arc of the earth's surface being almost precisely 100 feet) and they exhibit themselves in the form of minute rotations of the pole, produced, like the precessional circle, contra-clockwise.

In another place I have sought to emphasize the circumstance that since Newton's day many fundamental scientific truths have come to light for which he made no anticipatory provision. Here is a case in point. Had he known of the existence of this phenomenon, in addition to all the other wonderful facts astronomy has unearthed since his time, it might have occurred to him to consider, at least provisionally, whether the torsions of the sun and moon upon the earth's equatorial ring could not better apply to this problem than to that of precession, for which he did employ them. He might then have raised in his mind the reflection that CYCLICAL CAUSES should produce CYCLICAL EFFECTS, and discarded, before he had gone too far, the absurd hypothesis that the annual attraction of the sun on the earth's ring produces a diminutive are of a big circle, rather than the diminutive complete circle it should naturally produce. In the new problem there is pressing need of the very causes Newton thus preempted for precession, but no astronomer dares to broach such a thing as the diversion of them to this new use, because the greater problem of precession would then be revived and make necessary a complete reconstruction and readjustment of the whole theory of the science-for which drastic course the powers that control are not yet ready.

So, instead of adopting the true reasons to explain the phenomenon in question, be the consequences what they may, astronomers are groping about for some plau- 
sible subterfuge that may serve to allay their troubled spirits and strengthen their faith in what they must know, deep down in their hearts, is not a science at all, but a pseudo science almost as visionary as astrology itself. I am speaking now of theoretical astronomy, remember, not of practical astronomy; for of the latter I have no words but of praise. We have just seen how, in the case of cometary tails, astronomers have invented light repulsion to take the place of the principle of equilibrium, which they wrongfully discarded, and we may now expect them to settle upon some equally grotesque theory to account for the present phenomenon so as not to disturb Newton's irrational explanation of precession. Lord Kelvin, Newcomb, and others have sought to explain the mystery on the basis of Newton's tidal theory by first deducing the coefficient of the earth's elasticity and employing this as a new basis on which to found further deductions. Others try to connect the anomaly up with continental risings and sinkings, with earthquake cycles, with atmospheric shiftings, with the alternating deposition and melting of snow and ice, and whatnot.

Paradoxical as it may sound to the ears of astronomers, it is Nот TRUE that the earth's axis shifts within her crust; nor is it true, as they imagine, that the cycles described are basically due to long-period gyrations. On the contrary, the phenomenon is produced by diurnal oscillations of the planet as a whole and the annual and 430-day periods are simply periodical changes in the amplitudes of those diurnal movements. An illustration may help to make the matter clearer: You remember how, in Foucault's celebrated pendulum experiment (by which he proved the rotation of the earth), the tip of the pendulum was made, at the end of each swing, to cut into a little circular ridge of sand. At the close of the experiment the pendulum tip left its tiny dents all round the circle as the only record of the oscillations, so that a stranger happening upon the scene after the pendulum had been removed might easily have supposed that the 
marks were caused by a toothed wheel rolled round the ridge, or in any way, indeed, save the right one.

Now, astronomical observations, especially in cases of such super-delicacy as required in this case, cannot be made at any hour of the day or night, but only at the most propitious time of all-midnight. That is to say, the marks or "indentations" are registered twenty-four hours apart-just as Foucault's pendulum's dents were spaced by a second or so. A record being kept of these latitude observations would speak only for themselves, but would give no hint as to what occured between their makings. Now, astronomers have mistakenly assumed out of hand that the condition between times is the same, or virtually the same, as at the moments when the observations are made-in other words, they see the dents in the act of being made in the circular ridge of sand, but they do not see, or allow for, the vibrations of the pendulum in between; they recognize the existence of the long-period variations, but remain blind to the even more significant diurnal ones.

We have only to suppose that the earth, besides its ordinary diurnal revolution, wabbles once around on its true axis during each day, and from day to day, in a rhythmical manner, with only the lengths of the amplitudes varying seasonally and monthly according to the changing positions of the sun and moon, to which latter we naturally turn for the dynamical explanation. Thus may we combine consistently the actuality of daily oscillations with Chandler's long periods of a year and 430 days respectively. These oscillations, be it observed, are not simple rocking motions, back and forth like a pendulum (which would not harmonize the phenomena), but a rotatory motion, which the term wabbling aptly describes. The conditions require that when $\mathrm{A}$ is on the meridian of midnight with a latitude plus, B at its midnight, twelve hours later, must show a latitude by the same amount minus. A wabble of a period of twentyfour hours, coöperating with the diurnal rotation, supplies this requirement. Were it possible to make accurate 
observations at all hours the same as at midnight, it would be found that both A and B shift their latitude all day long with a double wave-like motion, each being half the day above, and the other half below, its home parallel. This wabble varies in radius according to the season of the year and the position of the moon, hence the variational showings of long period.

What causes this phenomenon? is naturally the next question. I attribute it to the same causes that Newton erroneously employed to explain precession; only instead of referring the solar and lunar attractions specifically to the equatorial ring, I prefer to refer them to the more general entity of the earth's center of gravity. I am logically privileged to make use of these causes, because I have freed them by employing others to explain the phenomenon of precession.

The earth having been described already in these pages as a body seeking its lowest center of gravity with reference to the sum of the stellar attractions, it follows that that center is eccentrically located with respect to her center of figure; that is to say, the two do not coincide, but the c.g. is slightly north of the c.f. In this condition we have a repetition of the basis upon which I predicated the explanation of the moon's longitudinal libration (Consult, also, Chapter XIV). The distinction between that case and this, however, lies partly in the fact that the earth is supplied with mobile oceans, whereas the moon is solid clear through; and partly in the distances, sizes, configurations, and relative motions of the attracting bodies. The principle of purchase, or leverage, accordingly again comes into play, necessitating application of the rule of inverse cubes in passing upon the relative perturbations of the sun and moon-our old ratio of 1 to 2.25. That such a complicated combination of dynamical factors as this should produce diurnal oscillations of the earth seems to me self-evident, and I shall therefore not labor the point. Nor do I think it necessary to enlarge on the presence of the annual term found 
by Dr. Chandler's analysis. The 430-days term, however, demands elucidation:

Were the sun and moon always in alignment with the earth, and did they not move from their places, the sidereal day would be the same as the solar day and the "lunar day" (if we may permit ourselves this last expression for the sake of simplicity). In that case, there would be no fluctuation of latitude at all. But the moon is not stationary; she steadily gains over the imaginary position we have just pictured her in, a position, however, which is monthly realized when she is said to be new. Now, the time elapsing between new moon and new moon is known as the synodical month, and its length is 29.53059 days, whereas the sidereal month is only 27.32166 days, consequently the latter in one year gains on the first 2.20893 times as many days as 27.32166 days is contained times in $365 \mathrm{r} / 4$ days. Performing the operations, we obtain 29.5 days, which, because the moon's torsional effect is 2.25 times as great as the sun's, must now be multiplied accordingly, yielding 66.4 days. Adding this to the annual period, we have 431.6 days, which is a very close approximation to Dr. Chandler's second term, considering the many uncertainties and difficulties involved.

A very interesting, not to say significant, coincidence brought out by this avenue of investigation deserves to be mentioned. If we average Dr. Chandler's four fractions (125-1000 sec.) and the two periods (398) days, and multiply them together-this on my hypothesis that the variation is of diurnal causation-we obtain $49^{\prime \prime} .75$, a quantity startlingly near the $50^{\prime \prime} .2$ are annually added to the precessional circle! 


\section{VIII}

\section{THE NEBULAR HYPOTHESIS}

$\mathrm{W}$

HAT militated most against the earlier acceptance of Newton's doctrine of the universality of gravitation was the unreadiness of his generation, accustomed though it was to the acceptance of ideas on faith, to believe that the force of gravity, any more than any other force of which they knew, could act across a void. It took the world of science half a century to assimilate this generalization, familiar though it may now seem to us. Unfortunately, the very fact that, in spite of its initial season of incredibility, it nevertheless won acceptance in the end, became a powerful lever in support of wild hypotheses whose only merit lay in their novelty. Since then, a premium seems to be placed on extravagance in invention, regardless of the element of probability or plausibility, and the only rule that appears to be recognized is, that inherited prejudices shall not be antagonized or impugned. All comers are welcome who will lend a hand at shoring up or patching up the superstructure of theory, but woe to such as I who would raze the crazy structure, in whole or in part, with intent to broaden and strengthen the foundations. In this chapter and the next my purpose is to give the reader as clear an idea as possible of the various cosmogonical theories that the hierarchy of science recognizes as orthodoxically sound and eligible, and then to criticize them from a practical, rather than a technical, standpoint. 
The most famous name in the annals of astronomy since Newton is that of Pierre Simon, Marquis de Laplace (1749-1827). The greatest debt we owe him, in my estimation, is not his so-called Nebular Hypothesis, not his mathematical contributions to astronomical science, but his specific and authoritative confession that Newton's system is deplorably and fundamentally deficient. I feel sure that the laity of the present day labor under the impression that the foundation provided by Newton was sufficiently broad and substantial to support the entire superstructure of the science for all time, but such is very far from being the case, as professional astronomers well know. In fact, it is astonishing how little Newton really did explain, when we come to compare his actual achievement with what still remained to be accomplished; verily was he right when he said, "I know not what the world will think of my labors, but to myself it seems that I have been but a child playing on the seashore; now finding some pebble rather more polished and now some shell more agreeably variegated than another, while the immense ocean of truth extended itself unexplored before me." Here are a few of the many things he did not explain:

The origin of the earth's rotation on its axis, and the reason for its continuance.

The origin of the earth's "rectilinear motion", and the cause of its continuance.

The origin of the axial rotations and orbital motions of the other planets and of the moon.

The origin of the sun's axial rotation and equatorial acceleration.

The genesis of the sun and of the planets.

Why the moon does not fall in upon the earth, or the earth and planets upon the sun.

Why the orbits of all the planets are nearly circular.

Why the planets rotate on their axes in the same direction.

Why they all revolve around the sun in the same direction. 
Why they revolve so nearly in the same plane.

What comets are.

Why these revolve in elongated orbits so dissimilar to planetary orbits.

Whether the sun moves in space, and why, and how.

What effect the stellar attractions exert upon the solar system, internally and externally.

The source of the sun's heat.

The nature of the stars and their mutual relations.

The cause of the moon's secular acceleration.

The origin of the earth's equatorial ring and her continental configurations.

It was to supply some of the deficiencies above enumerated that Laplace invented his celebrated Nebular Hypothesis, which he first announced in 1796, and twelve years afterwards republished, with certain corrections and additions. In brief, his scheme was this:

The solar system originally existed in the form of a gaseous nebula that extended out from the center of the sun (which he regarded as stationary) to somewhat beyond the orbit of the outermost planet (at the time of his death Neptune was yet undiscovered). This nebula had an inherent motion of rotation around an axis at right angles to its plane and passing through the center of the sun. At first the nebula was probably spheroidal in shape, but by virtue of its axial rotation it was gradually flattened out into lenticular form. In the course of time the rotary motion, by reason of the contraction of the mass, became accelerated, so that finally the nebula spun around so swiftly that it cast off an outside ring. Relieved of the weight, the residuum revolved all the faster, and a second ring was cast off. This process continued then until as many rings had been thus shed as there are planets, and there finally remained only the central residual mass which now constitutes our sun. This latter, being eternally hot and bright, naturally suggested to Laplace that the nebula was incandescent to begin with and retained its temperature throughout the process, an inference all the more plausible because the in- 
terior of the earth, also, is known to possess a high temperature.

The several rings thus thrown off (or abandoned, as some insist) in good time gathered into balls of nebulous matter possessing axial rotations, like the parent nebula, and, following precedent, likewise exchanged the spheroidal for the lenticular shape and eventually flung off smaller rings, which later developed into satellites.

Granting the premises, then, Laplace, up to this stage, had ostensibly accounted for these things: first, the revolution of the planets in the same direction; second, their revolution in (about) the same plane; third, the near-circularity of their orbits, and, fourth, the solar heat.

He had still, however, to account for the fact that all the planets rotate, like the earth, on their axes, and in the same direction in which they orbitally revolve. To do this, he gratuitously assumed that the nebula revolved in hydrostatic equilibrium, that is to say, like a rigid solid. His reason for so doing was this: the planets roll on the perimeters of their orbits like wagon wheels along a cartway, that is to say, their distal halves (as viewed from the sun) move forward while their inner halves move relatively backward. It was essential, therefore, for the sake of mechanical consistency, to depict the outer edge of the cast-off ring as traveling faster than the inner edge, just as the circumference of a flywheel travels at a faster rate than the inside of its rim.

As the data stood during Laplace's life, the Hypothesis correlated them all quite faithfully, a circumstance which doubtless accounts for the amazing hold it so long retained upon the scientific mind. The first notable departure from it came in the fifth decade of the past century, when heat was identified as a mode of motion. However, this departure was more in the nature of a development or vindication than a contradiction, inasmuch as it relieved the theorists from defending the absurdity of the nebula's maintaining its temperature in the face of the cold of space. But the day of disaster ir- 
retrievable was on its way. In 1877, Prof. Asaph Hall, of Washington, discovered the two moons of the planet Mars, Deimos and Phobos, neither one of them more than seven miles in diameter, and incidentally ascertained that the latter (and inner) of these revolves around its primary more than three times as rapidly as the planet rotates on its own axis. Some five years later, Keeler proved, spectroscopically, what Clerk Maxwell had previously shown deductively, that the rings of Saturn are neither liquid or solid, as theretofore supposed, but consist of multitudes of minute satellites, and that the inner ones revolve faster than the planet rotates on its axis. These two incompatible facts, added to the more recent discoveries of the so-called retrograde motions of three or four of the satellites, and taken in connection with its violation of the doctrine of conservation of moment of moments, have virtually given the Hypothesis its quietus.

\section{Criticism of the Nebular Hypothesis}

You may ask, of what use is further criticism of Laplace's Hypothesis if the death blow has already been administered? My answer is, that science has not yet sufficiently learned the lessons the career and final fate of the Hypothesis are capable of teaching. The truth is, it should never have required killing; it was too fatuously and inherently absurd on its face to have merited more than passing consideration. Of what use is it to postulate the axiomatically impossible and then to ring the changes on it for a century, only to learn in the end, by painful experience, what was but too glaringly patent at the outset? I give everybody out of Bedlam credit for knowing, a priori, that a gaseous nebula six billion miles across, far thinner than atmospheric air, could by no possibility, whether in the past, the present, or the future, rotate like a solid, spontaneously and without motive power, for thousands of years on end! If our intelligence cannot teach us obvious things like this, it can teach us nothing, and we may as well give up all efforts at learning. Should scientists have needed to await the discovery 
of Phobos in order to learn from that midget so rudimentary a lesson? But let us expose to ourselves the full extent of our recent folly in the hope of deriving wisdom not to go on perpetrating similar lunacies in the future.

To begin with, what think you was the degree of density of the postulated nebula? This is easy of calculation. Spheres being to each other as their cubes, and Neptune's distance from the sun being 6400 times the solar radius, it follows, that when the nebula filled the Neptunian orbit, every cubic inch of the sun's substance was dispersed in a cubical space 533 feet each way, that is to say, a space whose six sides are each equal to a largesized city block! Imagine a great air-tght box of this magnitude, filled with air of sea-level density, and then imagine this air pumped out and condensed into liquid form; it would fill a hall sixty feet each way. Reserve but two tablespoonfuls of this liquid (pouring all the rest away) and heat the same as hot as your imagination can picture, and then inject it back again into the big exhausted space from which it was pumped, and you will have a mathematically accurate idea of the nature of the Laplacian nebula! Physicists inform us that, despite their utmost care and ingenuity, they are unable to produce artificial vacua within 1-1000 part as tenuous as this. Let fall from your eyes, I pray, the glasses of prejudice and look, and, looking, do some sane and sincere thinking. Do you, in your inmost soul, believe that such a hypervacuum could and actually did rotate on its axis LIKE A RIGID solID and, so rotating, fling off great rings by centrifugal force? Have you in all your reading come upon a fairy story, a superstition, a dream, an hallucination so utterly abandoned and nonsensical as this? Yet for nearly a century has this absurdity been taught by a science that prides itself upon being the only "exact" one outside of mathematies! If this be astronomy, then let astrology be hailed as true gospel.

Let us now inquire what is the extent of the sun's attraction at the distance of Neptune, in order to ascertain the degree of "hydrostatic pressure" brought to bear on 
the material of Neptune's ethereal ring. Not to weary the reader with too much detail, let me give a few calculated results. The attraction of the sun on a given particle as distant from him as Neptune is about one fourteen-hundred-thousandth as great as that of our earth on a like particle at her surface. It is only about one-fourth as great as that of the moon upon the ring on your finger, and is proportionally about equivalent to

- that of the attraction of your own body upon the clock resting on the mantel a few feet from you.

Now, gravitational attraction is one thing, but tangential torsion by it is altogether another. It is quite admissible to conceive of the particles of which we are speaking as being held back by their gravitational attraction from escaping into outer space; but it demands the maximum of credulity, scientific or otherwise, to believe that the friction between particles so sparse and minute as here demonstrated, and cohering only by grace of a vivid fancy, could ever, under any circumstances, sustain a general torsional motion. To refer again to our illustration, this friction is relatively the same as the act of turning around on your heels (while still preserving the intervening distance) would have on the clock, not to draw it toward you, remember, but to $r u b$ it to one sideno, even less, because the air intervening between the clock and you is many thousands of times denser than the postulated nebula, and a proportionately stronger frictional medium.

Again, Laplace made no attempt to explain how his unique nebula came into existence-he merely postulated it. According to modern molecular theories, be they true or false, the molecules are perpetually seeking freedom and to scatter themselves as thinly as possible through the wide reaches of space. Yet here we have our doctrinaires soberly telling us, that not only did the Laplacian molecules, without any particular reason, congregate into a vast heap, but that they cohered to each other with a tenacity many thousands of times more firm and rigid than the staunchest steel! Moreover, after the rings 
severally parted, why did they not continue in the "solid" state, seeing that they were then relieved from torsional strains, instead of immediately dissolving, and later reuniting, as Laplace described; and, once dissolved, why and how did the minor nebulous masses manage to reacquire the "solid" attributes in preparation for the casting off of the secondary rings? Yet not for plain, practical, common-sense reasons like these have scientists thrown off the Laplacian incubus, but for such relative trivialities as the behavior of little Phobos; that cosmic gnat upon which science foundered after banqueting upon this caravan of French-served camelopards !

In short, the Nebular Hypothesis of Laplace removed no difficulties whatsoever, but only set up futile others. In its initial statement he frankly assumed, as much as did Newton himself, a physically uncaused motion for his matter; second, he assumed its incandescence, despite its envelopment by the unspeakable cold of space; third, he postulated an unnatural cloud-form; fourth, he made no attempt to explain how the nebula originated; fifth, he took no account whatever of the fatally disturbing factor of the sun's motion through space; and, sixth, granting him all his egregious postulates, the machine he invented was geared too high to keep going, even had it ever got started. To speak frankly, the Hypothesis bore upon its face such crass imbecilities as should have condemned it instanter. Even had it provisionally solved all the details it was intended to do, its palpable and inherent defects should have discredited it nevertheless, and such apparent responsiveness been laid to the credit of mere coincidence. As it has turned out, science has frittered away a century in laboriously putting this grotesque wraith through a series of imaginary gymnasties, only to have it perversely turn and twist the wrong way like a double-jointed harlequin. In its application to details the Hypothesis has been found consistently disappointing.

From Laplace down to Mayer and Joule, scientists peacefully believed in the possibility of this nebula being superheated; but no sooner did the Mechanical Theory of 
Heat appear than they confessed their previously concealed misgivings and welcomed the newcomer with open arms. With a great sigh of relief they improvidently cooled with its breeze the nebula's incandescence down to the zero of space, and began to build with the chilled embers the burning sun and molten worlds. New phrases, such as "kinetic energy", "energy of position," etc., sprang up, which, interpreted, mean, that by the mere coming together of the particles of the nebula under the constraining influence of gravity, heat was produced by their mechanical impacts sufficient to melt the substance of the forming planets, and to endow the sun with a supply of caloric capable of lasting that prodigal milliards of years. Thus science rested in smug content until Helmholtz, the great physicist, fortified the theory with his idea of a slowly contracting sun, so that certain sleepers who had given some signs of waking were lulled into still deeper slumber.

Of late, however, it has begun to dawn upon scientists that their theories involve the ultimate destruction of the universe by the dissipation of all heat, when the last impact shall have sounded, and all the worlds shall finally have been gathered into a single inert mass. In the succeeding chapters I shall endeavor to disprove this dismal forecast.

In order to convey to the reader in as concrete and graphic a form as possible the current scientific notion of how the earth's internal heat came about, as well as the heat of the sun and the major planets, let us imagine the substance of the sun divided into flakes, say a hundred to each cubic-inch of matter. It is a pet idea of modern science that the farther apart the particles of matter are, the greater their "energy of position"; so let us meet her views, as nearly as we know how, by picturing the nebular field divided into cubical chambers 100 feet each way, and allot to each chamber one such flake-its full share. Now, although Laplace assumed these flakes (of course he did not use this particular illustration) to be incandescent, modern science magnanimously admits they 
could not have been so, exposed as they were to the absolute zero of space. But, says science instead, these flakes immediately began to attract each other and to cause their mutual collision with such force as not only to keep themselves and their neighbors warm, but to store up so much excess heat that after several hundred millions of years the four major planets are still in a molten state, and the earth's interior so hot as to melt granite and every instant to threaten her cataclysmic disruption! Assuming that the earth was actually formed in this manner, that the process took the moderate period of five million years (which is much less than scientists ordinarily allot), and that the accretions were gradual and uniform, figures will show that the average daily sprinkle in the earth's case could not have exceeded one-fourth of an inch! When, in addition, we take into account that the flakes and the planet itself were continually exposed to a far colder than arctic temperature, and that the sun had his own future to look after the while, one begins to feel, does he not, that the effects of this cosmic snowstorm have been somewhat Munchausenized?

But here I may be accused of unfairness in choosing the simile of a snow-storm, as no doubt the substance of the nebula was more compact than that, say in the form of meteors. To this I answer, first, that Laplace specified gaseous matter, which is vastly lighter, and, second, that, even so, the larger the particles the fewer the impacts and the farther between, hence the arithmetical aggregate would be just the same; but, fortunately, there is a second answer, which may fairly be held to be experimental in character; and in weighing it let it be remembered that the earth is now at its maximum of attractive power. I quote verbatim from Professor Percival Lowell's book, The Evolution of Worlds (p. 41). He says:

Most meteorites are stones, but one or two per cent are nearly pure iron mixed with nickel. When picked up they are usually covered with a glossy thin black crust. This overcoat they have put on in coming through our air. Air-begotten, too, are the holes with which many of them are pitted. For, entering our atmosphere with their speed in space is equivalent to im- 
mersing them suddenly in a blowpipe flame of several thousand degrees Fahrenheit. Thus their surface is burnt and fused to a cinder. Yet in spite of being warm to the touch their hearts are still cosmically cold. The Dhurmsala meteorite falling into moist earth was found an hour afterwards coated with frost. Agassiz likened it to the Chinese culinary chef d'oeuvre, "fried ice". It is the cold of space $200^{\circ}$ or more Centigrade below zero, that they bear within, proof of their cosmic habitat.

I ask, How many such meteoritic impacts as this of Dhurmsala at $200^{\circ}$ below zero would be required to make up a sun as hot as ours? or how many million years would be required for them to "cool" up to the molten condition of the major planets?

Here is another piece of testimony from the recent work of Professor T. C. Chamberlin, of the University of Chicago, The Origin of the Earth, (p. 163):

Meteorites, even after they have plunged through the whole atmosphere and into the earth, are said sometimes to retain a very low temperature within. They are reported even to freeze the earth in which they imbed themselves. At any rate, the low temperatures brought in from space must be set over against the heat of atmospheric friction in the ledger of temperature effects. Very significant, in this respect, is the almost incredible existence of a small class of meteorites largely formed of volatile and combustible hydrocarbons. These have reached the earth without either complete vaporization or combustion.

The shorteomings of the collisional theory became early apparent, so that, in the year 1854, Helmholtz was led to propose a new explanation of the solar heat. Discussing this theory Doctor Abbot says (The Sun, p. 277):

Helmholtz pointed out that the shrinking together of the sun converts potential energy of position finally into heat. Several authors have made computations of the quantity of energy which would be available from this source. Their results have generally been based on the assumption that the sun was originally a nebula filling a sphere whose diameter was the orbit of Neptune. It appears that the condensation of such a nebula having the mass of the sun would have furnished thus far about $25,000,000$ times as much energy as the sun now loses each year.

According to Helmholtz's view, a contraction of about 250 feet per year in the sun's diameter would suffice to sustain the present solar radiation. At this rate it would require about Io,000 years to reduce the apparent diameter of the sun by one 
second of arc, so that, so far as telescopic observation is concerned, the contraction theory is tenable, for a change of $\mathrm{I} / \mathrm{IO}$ second in the solar diameter is unrecognizable. From calculations of Newcomb the sun will require to have shrunk to onehalf its present size if it maintains its present rate or radiation for about 7,000,000 years longer. 359) says:

On the same subject Doctor Young (Genl. Astr., Art.

As to the past of the Solar history on this hypothesis (Helmholtz's), we can be a little more definite. It is only necessary to know the present amount of radiation, and the mass of the sun, to compute how long the solar fire can have been maintained at its present intensity by the processes of condensation. No conclusion of geometry is more certain than this,- that the contraction of the sun to its present size, from a diameter even many times greater than Neptune's orbit, would have furnished about $18,000,000$ times as much heat as the sun now supplies in a year, and therefore that the sun cannot have been emitting heat at the present rate for more than $18,000,000$ years, if its heat has really been generated in this manner.

But this conclusion rests upon the assumption that the sun has derived its heat solely in this way, and the recent discoveries with respect to radium and radio-activity strongly suggest other causes which may have added large contributions, and may still be operative in maintaining the solar radiation.

Apropos of the same subject we may add this from Doctor Newcomb (Art. Sun, Americana):

If the sun were merely losing energy like an ordinary hot body cooling off, a very simple calculation will show that it would be so cooled off in the course of 3,000 or 4,000 years as no longer to radiate much heat. It is clear that such has not been the case. Yet the most careful study shows no possibility that it can be receiving energy from any outside source. Moreover, the geologists assure us that the stratification of the rocks, as well as many other phenomena associated with them, proves that the sun has been radiating heat to the earth at not much less than its present rate for hundreds of millions of years.

How fertile in expedients, these astronomers, and how well they love to eat their cake and have it too! Consider, if you please, these facts: Away back in 1853, when Helmholtz put forth this explanation, the Laplacian hypothesis was in its heyday, and not a voice in the land 
of science was there heard against it. At that time it was regarded as "one of the most well-attested facts of human knowledge". Now, if there was anything clear about that conception, it was, that absolutely the whole of the matter that composed the Neptunian annulus went into that planet and his satellites; so in the case of the Uranian annulus, and so in turn with all the planetary annuli down to and including Mercury's. Yet in all the estimates I have come across in my reading, I have failed to find a single one that does not construe the Helmholtzian sun as having contracted from the very periphery of the original nebula. What is the meaning of such crass misrepresentation? Is it due to innocent oversight, or to stupidity, or to intentional duplicity? Be the reason what it may, the truth remains that the sun cannot be consistently predicated as having collapsed upon itself from a greater distance than from the line of Mercury's orbit. Now, Mercury is only 1-80 as far from the sun's center as Neptune, and if we take the square root of 80 (to allow for gravitational variation) and divide Young's estimate by that quantity, the life of the sun becomes theoretically reduced to a paltry $2,000,000$ years!

The situation is in no way improved by postulating a meteoric in lieu of a gaseous nebula, should such a substitution be attempted. In the contemplation of science the original nebula, however constituted, possessed a concerted movement of rotation about an axis, and there is no logical warrant for asserting that part of it took on orbital motion while the rest was free to fall. Even conceding that this last may have been the case, that part which was free to fall would inevitably have traversed the distance from Neptune to the sun within a few years at most, and its collisional effects been ages ago dissipated. Indeed, it was precisely to escape this galloping culmination that caused astronomers to look with so much more favor on the gaseous hypothesis. In all cosmological speculations, duration is of the essence, and particularly is this the case here. So far, then, as the Helmholtzian explanation of the solar heat is concerned, the 
only feasible hypothesis is that the primordial nebula was strictly gaseous and that the sun contracted to his present volume from a maximum radius no greater than thirty million miles, equivalent to a radiating longevity of less than 3,000,000 years, liberally estimated.

So much for the quantitative deficiency of the contraction theory, now as to its qualitative merits. Although all astronomers, it appears, accept the theory, there is a division of opinion among them as to whether the sun is getting hotter, as time goes on, or colder. Such an elementary disagreement as this certainly does not inspire confidence, especially when neither view accords with the phenomena demanding elucidation. The sort of sun geology prescribes is one which, though having evidently suffered many vicissitudes, has nevertheless managed to preserve a rather uniform average of temperature throughout geological time. That is to say, if we conceive geological history to be divided into periods of equal duration, ancient, medieval and modern, the mean temperature of the sun during each of these was virtually the same as it was in the others. A secularly cooling or warming sun, such as Helmholtz's hypothesis contemplates, even were it quantitatively sufficient,.is therefore qualitatively unacceptable. Geologists, moreover, inform us that genial and frigid ages have alternated with fair regularity in each of these enormous periodsanother essential qualification on which the Helmholtzian lamp casts no light.

Until about the year 1880 it was supposed as a matter of course that because the sun is, in theory; conceived to be shrinking on account of his cooling, his temperature must be consequently falling. It was reserved for J. Homer Lane, however, to propound the weird paradox that the more that luminary cools the hotter he gets! I quote Sir Robert Ball's exposition of the idea (Story of the Heavens, p. 522):

And now for the remarkable consequence, which seems to have a very important bearing on astronomy. As the globe contracts, a part of its energy of separation is changed into heat; that heat is partly radiated away, but not so rapidly as it is pro- 
duced by contraction. The consequence is, that although the globe is really losing heat and really contracting yet that its temperature is actually rising. A simple case will suffice to demonstrate this result, paradoxical as it may at first seem. Let us suppose that by contraction of the sphere it had diminished to onehalf its diameter; and let us fix our attention on a cubic inch of the gaseous matter in any part of the mass. After the contraction has taken place each edge of the cube would be reduced to half an inch and the volume would therefore be reduced to oneeighth part of its original amount. The law of gases tells us that if the temperature be unaltered the pressure varies inversely as the volume, and consequently the internal pressure in the cube would in that case be increased eightfold. As, however, in the case before us, the distance between every two particles is reduced to one-half, it will follow that the gravitation between every two particles is increased fourfold, and as the area is also reduced to one-fourth, it will follow that the pressure inside the reduced cube is increased sixteenfold; but we have already seen that with a constant temperature it only increases eightfold and hence the temperature cannot be constant, but must rise with the contraction.

What sophistries are perpetrated in the name of Science! Here we are told, in so many words, that to warm the sun the way to do is to cool it, and in order to cool it we must heat it. Let us take the statement exactly at its face value and see how it works out in practice.

The world of science is pretty well agreed, I think, that the chemical constitution of the earth and the sun is much the same, and that if there be any appreciable difference at all, the sun should possess a higher percentage of the heavier materials than the earth. Nevertheless, the sun's density - that is, his ratio of mass to volumeis very much less, being only 1.4 that of water as against the earth's 5.5, and a simple calculation will reveal that in order for the former to become as compact as our globe, he shall have to contract his diameter by 312,000 miles.

Now, why is he so distended? Surely not because of his axial rotation; for, according to accepted ideas, were centrifugalization the cause, he should possess an immense equatorial protuberance, whereas he exhibits no oblateness of figure at all. There is but one answer, namely, it is because of his exceedingly high temperature. 
But if Lane is correct in asserting that the sun's temperature is rising, then his volume must be expanding from the effects of the increasing heat, and there follows a double paradox, namely, that besides growing hotter because he is cooling, he is expanding because he is shrinking.

On the other hand, if the Laneites are in error, it does not necessarily follow that those who claim the sun is falling in temperature in the process of shrinking are right. The sun may, in fact, not be shrinking at all, but simply holding his own! For let us hark back to the time when the luminary may be supposed to have possessed double his present diameter, and to have had a temperature, say, twice as high as at present. Then, according to Stefan's law, his radiation, area for area, must have been increased in the ratio of the fourth power, or sixteen times, and his surface area being concurrently multiplied by four, his total radiation must, according to figures, have been 64 times as copious then as it is to-day-obviously contrary to the biological facts. Indeed, the sun dare not even be postulated as at that time possessing the same temperature as now, inasmuch as he would then have been radiating four times as much heat, on account of his greater area;which would have meant the total destruction of all terrestrial life then extant.

That the Helmholtzian hypothesis leaves much to be desired is well recognized now, though scientists were loth to admit it prior to the discovery of radium in 1898, when a new hope dawned. Since then; zealous efforts have been made to discover traces of radium in the solar spectrum, but so far without success. Professor S. A. Mitchell, of Yerkes Observatory, writing in 1913 (Pop. Astr., No. 206), reports thus:

From theoretical considerations we are positively convinced that there must be radium in the sun. But to prove this is another problem. With the spectra we already have we can prove nothing more than accidental coincidences. One of the problems for the solar eclipse of August, I9I4, will be to obtain the spectrum of the chromosphere on a large scale with good definition, so that we may prove what we think we know, that radium is in the sun. (Later eclipses have developed nothing new). 
If I interpret this quotation correctly, here is an intimation that if ever astrophysicists succeed in finding even so much as an indubitable trace of radium existing in the sun, the whole vexing problem of the source of the solar heat will be labeled "solved" and put away on the shelf for keeps. But the radium hypothesis can never solve this problem finally, no matter if the sun be proved to contain any desired percentage of radium, or radioactive materials. Even if it successfully disposed of the quantitative question, it would still leave all the qualitative difficulties untouched. But it does not by any means measure up to the quantitative requirements. Professor Joseph Barrell, in a recent Bulletin of the Geological Society of America, says:

But the sun is only one of the numberless host of stars, and the source of its energy is a far-reaching cosmic problem. To warm the earth through the vast length of geologic time, gravitational condensation of the solar mass is found to be totally inadequate. The energy supplied by the atomic degeneration of uranium and thorium would have ample endurance in time; but, even if the sun were composed entirely of these elements, their decay could not supply the quantity of energy which is daily expended. Geologic time brings to light, consequently, the evidence of unknown sources of energy, cosmic forces which must constitute a fundamental factor in any satisfactory hypothesis of stellar evolution, a factor which has not as yet been taken into full consideration in its bearings. Even if, as a lesser diffculty, it should be sought to deny the validity of the radioactive measurements of the earth's age, escape cannot be had from this conclusion, for various lines of purely geological evidence indicate an age many times greater than that which could be granted if the solar energy were due simply to contraction of the sun's mass. The depths of geological time leave us face to face with the unknown.

One of the most baffling problems of the geologists is to explain the now well-established periodical recurrence of ice ages throughout geological history. As to these I quote from the pen of Professor T. C. Chamberlin of the University of Chicago. He says (Origin of the Earth, p. 4):

But this theory of a simple decline from a fiery origin to a frigid end, from a thick blanket of warm air to a thin sheet of 
cold nitrogen, consonant with the current cosmogony as it was, logical under the premises postulated, pessimistically attractive in its gruesome forecast, already in possession of the stage, with a good prospect of holding it-this theory of a stupendous descensus none the less encountered some ugly facts as inquiry went on. It seemed to accord well enough with an ice age, if the ice age came only in the later stages of the earth's history, but it was ill suited to explain an ice age in the earlier geologic eras. Unfortunately for it, there began to appear signs of ice ages far back in time, and, besides, some of these had their seats much nearer the equator and, in other respects, were even stranger than the latest great glaciation. The evidence of these earlier and stranger glaciations was at first quite naturally received with incredulity, but the proof grew steadily stronger with every new test, and the range of the evidence was found wider and clearer as exploration advanced. While all this should have weakened, and did weaken, the fundamental concept of great warmth and a rich atmosphere in the earlier ages, while it should have roused skepticism as to the verity of the cosmogony on which it was based, and perhaps did so, still the old thermal concept and the old cosmogony continued to hamper all attempts at a radical revision of glacial theories.

Again. Summing up a luminous chapter on the numerous theories as to the causes of ice ages, Professor W. B. Wright, in his monumental work, The Quaternary Ice Age, condemns them all as worthless. He says (p. 451):

It must be admitted that, among the theories that have been brought forward to account for the phenomena of the Ice Age, there is not a single one which meets the facts of the case in such a manner as to inspire confidence. An almost fatal objection to Croll's theory is the date it assigns to the end of the Ice Age, which it places some 80,000 years back. If as Doctor Geer seems to have clearly established the ice margin retreated north past Stockholm only about 9,000 years ago, this practically excludes any possibility of a connection between glaciation and changes in the eccentricity of the earth's orbit. 


\section{IX}

\section{RECENT COSMOGONIES}

\section{The Capture Theory}

K

EPLER'S second law, namely, that the radius vector of the several planets passes over equal areas in equal times, has since been generalized to include all circulating cosmic bodies, and is usually referred to as the law of the conservation of areas, or the law of the conservation of moment of momentum. It appears that through some unaccountable oversight neither Laplace or those who came after him ever thought of applying this test to the Nebular Hypothesis until the year 1861, when the French physicist, Jacques Babinet (17941872), contributed an article to the Comptes Rendus, in which he showed that the Laplacian conception grossly violated that requirement, and that when the sun was supposedly expanded to the orbit of Neptune, or to the orbits of any of the nearer planets, the rotation was much too slow to detach any of them by centrifugal force. At that time, however, such was the prestige of Laplace and his cosmogony that Babinet's work received but scant consideration, and even Babinet himself kept on teaching the same old fallacy, notwithstanding, until his dying day; much as Sir George H. Darwin did the Newtonian tidal theory, which he himself had so conclusively disproved.

About a half century later, Doctor T. J. J. See, the world-renowed astronomer and Director of the U. S. 
Naval Observatory at Mare Island, California, in the course of his extensive researches came upon this longforgotten article of Babinet's and brought it again to the attention of the scientific world. Even then it would have received no consideration, it is safe to say, either from Doctor See himself or from other professional scientists had the Hypothesis not in the meantime become discredited by the discovery of Phobos, the resolution of the Saturnian rings into discrete particles, and the revelation of the existence of retrograde motions in the system.

Adopting Babinet's criterion as final and conclusive, Dr. See reasoned that since the planets and satellites could not possibly have been flung off by the centrifugal rotation of the solar nebula, there was but one other way by which they could have become members of the sun's family, and that was by capture or, in other words, by falling inward toward the sun from a great distance. If, he argued, the sun could directly form out of the cosmic dust, why not the planets, satellites and comets, severally and independently? It requires but little reflection to see that to give plausibility to this scheme, if for no other reason, the original nebula would have to be expanded to vastly greater dimensions than that of Laplace, and accordingly Dr. See himself affirms, that "it was between one trillion and five trillion miles in radius."

It was not only obligatory but natural for Doctor See to seek to correlate, as part of his cosmogonical scheme, all the modern developments of the science. One of these innovations was, of course, the doctrine of light and electrical repulsion. Laplace, as you know, had taken his nebula for granted; but this, Dr. See supplies by derivation from the impalpable dust expelled from the stars "gathering into clouds in the opposite parts of the heavens." His nebula, then, differs fundamentally from that of Laplace in being of the meteoroidal and not the gaseous type-a very material distinction, especially in relation to the applicability of the Helmholtzian explanation of the solar heat.

In Chapter I (q. v.) I quoted from Spencer and Fiske, showing how they respectively sought to account 
for the rotation of the Laplacian nebula-the former, by its direct condensation, and the latter, by the resolution of its multitudinous interior conflicting currents into a uniform gyratory resultant. The primary desideratum in both these hypotheses, in the hypothesis of Laplace himself-in all the hypotheses, indeed, that center about this nebular conception-is not so much to account for the rotatory motion of the nebula, as such, as to supply the agency for starting the planets and satellites into concerted vortical movement. In brief, the ruling idea is that these bodies derived their motions immediately from their parent nebula as integral with it, and not from any independent, extraneous source. That Doctor See shares this view clearly appears from the quotation already given (v. p. 11), in which he says, inter alia: "Spiral nebulæ arise from the meeting or mere settling of unsymmetrical streams of cosmical dust. The whole system of particles has a sensible moment of momentum about some axis, and thus it begins to whirl about some central point, and gives rise to a vortex. ${ }^{*} * *$ The dust carried away from the stars by repulsive forces gathers here and there into clouds, and when such a mass settles the result is a cosmical vortex, which whirls and slowly develops into a system".

It will be remembered with what scrupulous care Newton strove to impress upon the world the notion that cosmic space is absolutely clear of any ponderable medium. Why? Because he recognized, even if his successors affect ignorance of it, the absolute necessity, under his theory, that the infinitely accurate adjustments between gravity on the one hand and the tangential velocity of the circulating body on the other, ordained, as he postulated, by the Creator, dare not on any account be altered, even in the slightest, without immediately and irretrievably destroying (not merely imperiling) the whole system. Since his day, however, a few phenomena, such as the moon's acceleration and the acceleration of Encke's comet, have come to light that have put the mathematicians sadly at a loss to explain gravitationally, 
save on one perilous assumption, namely, that the ether, or some other cosmic medium mingled with it, is opposing the free passage of the revolving bodies.

It may strike the lay reader as paradoxical to be told that the acceleration of an orbitally revolving body can be explained by its retardation, but the acrobatic astronomer of to-day is equal to any emergency. His explanation is, that a body so retarded in its orbit is thereby forced to fall to a lower level, where, he tells us, under the mystical law of conservation of areas, "it is natural for bodies to move and revolve faster than at greater distances.' Not only, says he, does the resisted body miraculously recover the velocity lost, but it actually acquires a tangential velocity greater even than at first! Such is "celestial"' mechanics!

In addition to thus cajoling themselves into believing that retardation makes for speed, just as Lane persuaded them that the sun, by cooling, becomes all the hotter, our learned doctrinaires, proceeding along the same lines, have succeeded in convincing themselves that a concomitant effect of this process is to cause the orbit of the retarded body to become more and more rounded.

This myth is the chief text and stock in trade of our author, who, in spite of his affectation of originality, is quite prosaically orthodox. He conceives the planets and the satellites, whose orbits are all subcircular, to have nucleated in the borders of the nebula, on the order of a trillion miles distant from the central sun, and thence to have descended through the nebular matter, which he defines as increasing in density toward the middle. At first, he says, their orbits were very elongated, but as they severally came nearer and nearer to the sun, they encountered stiffer and stiffer resistance from the nebular matter enveloping them, causing them to fall to still lower levels, and consequently to hasten their velocity and take on greater rotundity of orbit. To quote him again:

In the writer's recent researches on the origin of the solar system, however, it has been shown that the orbits of the embryo planets were originally hundreds and perhaps thousands of times. 
larger than they are now, and have since been reduced in size and rounded up by the secular action of the resisting medium formerly pervading our system. As the planetary orbits were once of vast extent, and have since contracted their dimensions, it follows that the embryo planets once formed a connecting lin $\bar{k}$ with the present system of comets. In the course of immeasurable ages, the planetary system contracted its dimension enormously, and at the same time eat out the interior portion of the primordial solar nebula; leaving about the sun and planets a hollow shell of nebulosity, from which, under the effects of certain mutual perturbations, fragments drop down to visit us occasionally. These are the comets, and this is why they come from the shell or envelope at a great distance from the sun, an observed fact which has puzzled astronomers and mathematicians for centuries.

In explanation of the axial rotations of the planets he says:

We may conceive that there is revolving about each planet a vortex of cosmical dust, some of which falls against the surface of the globe, and thereby accelerates its axial rotation. As the predominant motion of the vortex is forward, the planet's rotation naturally tends to become direct and the obliquity tends to disappear.

Doctor See's own appraisal of his theory can be gathered from this, his concluding paragraph:

The most remarkable fact about the capture theory of cosmical evolution is the harmony which it introduces among the most diverse phenomena. This shows that it rests on true causes and is approximately an ultimate truth, which may indeed be modified by future discovery, but will never be radically changed. It gives us a simple and natural conception of the processes by which cosmical systems are formed and unites and harmonizes all the known phenomena of the sidereal universe, so that, with the exception of universal gravitation, it is believed to embody the most ultimate law of the starry heavens.

\section{The Capture Theory Criticized}

Before proceeding to the more technical criticism of Doctor See's theory it may be well enough to get a clear idea of the nature of this so-called nebula with which he is conjuring. In considering Laplace's nebula, we found its density to be that of a cubical void 535 feet each way charged with the equivalent of but a single cubic inch of 
liquid air. Let us compare that density with the density of Doctor See's nebula. Taking the minimum radius mentioned by him, namely, a trillion miles, and dividing by the radius of Neptune's orbit, 2,800,000,000, gives the number 350, which being cubed reveals that See's nebula is, in round numbers, $43,000,000$ times as rare as Laplace's! We can get a more concrete idea of its extreme tenuity by multiplying the side 535 feet by 350 and erecting about this distance, as one edge, a larger cube, 35 miles each way. Such a cube would cover with its base the entire area of the State of Rhode Island, land and water. The air from such a cube, supposing it throughout of the density at sea level, if reduced to liquid form, would fill a cubical vat four miles each way (64 cubic miles). Discard this all, retaining but a single cubic inch of it, as in our former illustration, and restore it to the great void of 42,875 cubic miles out of which it came, and you acquire a mathematically accurate idea of the sort of nebula Doctor See is dogmatizing about. However, one distinction it is important to note, namely, that in place of using the substance air, which is a gas, we should substitute, say, a heaping teaspoonful of fine flour. Such, then, is the, by courtesy, "density" of the so-called "resisting medium" which Doctor See so trustfully adopts as the corner stone for his cosmogonical edifice, or shall we say, as the barrier on which he relies for turning back the charging planets and training them to course in rounded paths.

But Doctor See has been guilty of even a worse solecism: he has incautiously fallen into the selfsame blunder for which he (rightfully) criticises Laplace, and which, according to his explicit declarations, is the chief, if indeed it be not the sole aim, inspiration and justification for his own cyclopedically elaborated theory. Laplace's conception, he informs us, was fatally defective "because it violated the law of areas" (Kepler's second law), namely, that the radii vectores pass over equal areas in equal times. Let us see, now, if Doctor See's does not commit the same offense. 
In the first place, how does he theoretically get his planets into gyratory motion? I answer, by assuming them throughout their period of growth as being component parts of the nebula and partaking, as such, of its ensemble motion. What else can he mean than this whon he says: "When such a mass settles, the result is a cosmical vortex"? Again, if he does not mean this, then he has obviously failed altogether in accounting for the planetary revolutions at all, and it devolves upon him to find some independent explanation; failing in which latter quest he leaves the whole business in as dense a cloud of darkness as he found it.

Now, since, by premiss, the planets are obeying the law of areas, and since they are doing so because the nebular cloud in which they are immersed is so doing, how in the name of commonsense can the planetary body meet with any resistance whatsoever? If we assume that the body is traveling faster than the immediately surrounding nebulous matter, then it is transgressing the law of areas by going too fast, or else the nebulous matter is coursing too slowly to conform to the law. In brief, by the mere act of positing in his very first premiss the possibility of the planetary bodies encountering nebular resistance, he stultifies his whole scheme and treats the law of areas as a scrap of paper.

Proceeding thus, Doctor See "solves" nearly every imaginable enigma that has baffled astronomers in the past; but I shall not pursue the ungrateful task of criticising his weird speculations further than to cite a single sample of his art of deductive reasoning from his chosen premises :

The extreme roundness of the orbit of Neptune is a clear indication that this planet moved for a long time against a vast amount of nebulous resistance. Therefore, it is very improbable that our planetary system terminates with Neptune. In all probability there are several more planets beyond the present boundary of the system, some of which may yet be discovered.

Now it so happens that Mercury, the innermost planet, which ostensibly has had many thousands of times more schooling at the hands of this alleged resisting medium 
than did Neptune, is by far the most eccentric of any, whereas, next to Venus, Neptune's orbit is the most rounded. In fact, there is no element of our planetary orbits more irreducible to a regular rule than just this one of ellipticity, as you may see from a list of the planets arranged in the order of this peculiarity: Mercury, Mars, Saturn, Jupiter, Uranus, Earth, Neptune, Venus. Furthermore, we cannot decently concede that it is in any sense consistent with See's general hypothesis that Neptune, in particular, could ever have enjoyed the benefit of the resistance of any matter now incorporated in the sun and interior planets, for all of that matter must inevitably have preceded and beaten him in the race of centralization. Making allowance for this factor, it can easily be figured that the nebular density of the zone actually traversed by Neptune was a billion times less than the vacuousness already computed, yielding a result comparable to that of a single cubic foot of atmospheric air to the volume of our earth! And it is from this nothingness revolving, mirabile dictu, in the same direction, and at equal speed, with the planet, that Doctor See predicates a "vast amount of nebulous resistance!" And, finally, because he sub-consciously sees the preposterousness of his pretensions, he strategically diverts attention to an already general divination that other planets may one day be discovered beyond Neptune, as if laying in advance the foundation for a future claim to prophecy and corroboration should the expected come true.

\section{The Tidal-Evolution Theory}

It was in the year 1755 that Kant published his work on The History of Nature and Theory of the Heavens, in which he maintained that, because of tidal friction, the earth's axial rotation is slowing up and our day consequently lengthening. His argument was, of course, based on Newton's theory of tidal formation, and, granting the correctness of that, Kant was undoubtedly right. He was one of the very few who have had an inkling of the truth that celestial mechanies is essentially the same 
prosaic thing as everyday terrestrial mechanies and not the poetic-license monstrosity that Newtonianism and Laplacianism have pictured it. He looked upon the earth gemuinely as a physical object capable of being stopped or retarded like any other, given the natural forces; although it must be confessed he seems to have found nothing amiss in the silly notion that the earth perennially centrifugalizes her equatorial waters without the consumption of power. Though straining on the gnat, he experienced no difficulty in negotiating the camel.

Remarkable as it may seem to the uninitiated, astronomers have found a way to test Kant's conclusion. This they have done by comparing the records of ancient eclipses as far back as the 8th century B. C., almost three milleniums. The result, however, has been negative; demonstrating, according to Young (Genl. Astr., p. 105):

At present it can only be said that the change, if any has occurred since astronomy became accurate, has been too small to be detected. The day is certainly not longer or shorter by I-IO0 of a second than in the days of Ptolemy, and probably has not changed by I-IO00 of a second.

In recent years Sir George H. Darwin (1845-1913), more than any other single investigator perhaps, has made a special and practical study of the tides; and, like the rest of mankind, he fell naturally into the habit of viewing the universe through the spectacles of his specialty. There were three peculiarities about our earthmoon system that struck him as possibly susceptible of correlation under the tidal principle, and to the pursuit of this object he devoted many years of his studious life. These three peculiarities, according to his interpretation, were the following:

1. The moon constantly turns the same side toward us.

2. Her surface is obviously volcanic.

3. The earth is the only planet having a single satellite, and this satellite is the largest, relatively to its primary, in the whole solar system. 
Between Darwin pére and Darwin fils there lay an antipodal distinction. The former was a pioneer in the true sense, caring little for established opinions, but everything for the realities of Nature. The latter, on the contrary, gave the lie to Nature and servilely followed on the heels of Tradition. What chance, think you, has the cause of progress, when one such as he, having uttered the statement: "The equilibrium theory of tides is nearly as much wrong as possible in respect to the time of high water. In fact in many places it is nearly low water at the time the equilibrium theory predicts high water. It would seem then as if the tidal action of the moon was actually to repel the water instead of attracting it, and we are driven to ask whether this result can possibly be consistent with the theory of universal gravitation"-yet deliberately flew in the face of the truth and rushed on in the same old rut?

So Darwin, following perversely in the footsteps of the mistaken Newton and Kant, pursued the following train of hyper-speculative reasoning:

Since, according to Kant, the day is longer (or at least theoretically ought to be longer) than it was a millenium ago, there was probably a time millions of years ago when the earth rotated on her axis much more swiftly than she does now. Granting this supposition, there is no reason why we may not suppose, further, that she even rotated in from three to four hours; at least there is no one in a position to disprove it. However, this supposition is orthodoxically permissible; for, according to Laplace, the earth-moon system was originally a spheroidal nebula rotating on its axis, and, by common acceptation, it rotated faster and faster in the course of its shrinking. Suppose, then, that when the nebular earth had shrunk to a girth not much larger than it has at present, it had already formed a weak crust and, incidentally, was rotating in the brief period mentioned. In that case it is possible that the earth may have flung off the moon with sufficient violence to detach her permanently from the planet and make a satellite of her. It is not necessary to stipulate 
just how far away the moon was thus hurled, but for the sake of illustration we may suppose she was flung to a height of some 4,000 miles, and we may further assume that both parent and offspring were in the molten, or a quasi-molten, state. Under these circumstances the two bodies would doubtless raise great tides upon each other's surfaces and these protuberances would, by their eccentric attractions upon each other, retard the rotational velocities of their respective globes. This would bring into play the mysterious law of conservation of moment of momentum, and the moon would consequently go out further and further by way of compensating for her slowly expiring axial rotating energy, until, finally, that rotation would become reduced to a minimum and the satellite would turn the same face constantly earthwardas it does now.

Careful computations have demonstrated that on account of the relatively small mass of the moon, her temperature under theimpact theory could nothave excerded, at the very beginning, more than $0^{\circ}$ Farenheit. This finding is plainly incompatible with the observed volcanis rugosity of her surface. It is also to be noted that whereas, in the order of size, we have the earth, Mars, and moon, their order of roughness is, moon, the earth, Mars. By deriving the moon from the earth, then, we may theoretically endow the former with the same temperature as our 80-times more massive planet. When, now, we take into consideration the fact that the lunar gravity is only about 1-6 of the terrestrial, it is easy to see why the explosive effects on the satellite have been so much more telling than upon either Mars or the earth.

Inasmuch as the nebular earth did not give birth to the moon as early in the process of condensation as did the other planets to their satellites, but reserved its energies until it had attained a very compact stage and, under the law of moments, a commensurately high velocity, it was only to be expected that the child should prove the Titan it did. 


\section{Criticism of the Tidal-Evolution Theory}

Any theory that breeds enigmas faster than it dispels them is ipso facto false and worthless. Having explained, as he imagined, the moon's present posture by a series of assumptions on assumptions, Darwin should have gone further and proved all those assumptions. In this long series, not one of his assumptions is universally conceded by the astronomical profession-the Laplacian hypothesis is obsolescent, if not actually obsolete; the lunar volcanic theory is contradicted by a large and growing faction who insist that the lunar asperities are due to meteoric impacts ; the Planetesimalists assert that the internal heat of the planets is of later genesis than the original ingathering of the nebulous matter; and so on. In any event, it is quite as sensible and scientific to accept the moon's fixed attitude as an ultimate fact of nature, as to swallow a dozen other even greater uncertainties and incomprehensibilities along with it in the effort to appear wise. But let me show you the physical absurdity of this tidal-evolution theory by a simple calculation which anyone can follow and verify:

The mass of the earth is estimated to be about 6,000 , 000 quadrillions of tons, hence that of the moon, which is about 1-81 as large, is, in round numbers, 74,000 quadrillions. The question is, how thick would have to be a cable of steel, of the tensile strength of 40 tons to the square inch, in order to equal the gravitational attraction between the earth and the moon when the latter was supposedly at the earth's surface, as it must have been in order that it may have been flung off as Darwin describes. Dividing, first, by 40 gives us 1,850 quadrillions of square-inches as the area of the cross-section of the cable. Now, the formula for the area of a circle being $\pi \mathrm{r}^{2}$, performing the operation gives us the thickness of the cable, in round numbers, as 24,000 miles! That is to say, Darwin and all who agree with him (which is to say, all the scientific world, with very few exceptions) gravely assert that by reason of her slow contraction due to cooling the earth acquired so much increased axial velocity as to en- 
able her to sunder a steel cable more than thrice her own diameter! Did I say sunder? That is the wrong word, for the bond of gravitation may be strained, indeed, but not broken. When the moon was hurled, as alleged, to the distance of 4,000 miles from the earth and became a satellite, their mutual attraction was thereby by no means destroyed, but only reduced to 1-4 of what it was before, equaling still the strength of an elastic steel cable 12,000 miles in thickness, or 1-1/2 times the earth's diameter. From that point on outward, Darwin assumes, by convention, that the momentum of the moon possessed at the instant of severance persists undiminished forever, sustaining her in her orbit automatically; and then he goes on to explain how the gravitational attraction of the ansae, or tidal protuberances of the moon, lift her further and further away - by her boot straps, as it were.

The velocity from infinity, or parabolic velocity, is that which a body falling from infinity would acquire on reaching the cosmic body under consideration. Thus, a cannon-ball falling to the earth from infinity would acquire, according to mathematicians, a velocity of 6.9 miles a second. Conversely, in order that any object expelled forcibly from the earth shall never return to it again, it must depart with this same velocity of 6.9 miles a second. Now, according to Darwin's premises, the moon must have been flung off with just that speed, since it appears she is never to return to the earth.

The query here suggests itself, what was the nature of the energy that accomplished this stupendous cast? It could not have been heat, because Darwin lays the cause of the earth's axial acceleration to its having cooled and shrunken. Furthermore, it can be shown that as much flinging energy would have been required as there would be heat generated by the reverse process of the fall of the moon from infinity upon the earth; that is to say, more than enough to vaporize both of these great bodies completely. Comparing the moon to a projectile being fired from the earth, against the latter's retarding attraction, out of a suitable cannon, at the initial velocity 
of 6.9 miles a second, would have required a charge of smokeless gunpowder several times heavier than the satellite's own weight.

That Darwin was not, however, entirely devoid of the saving salt of common sense, in spite of the indications, is shown by his concluding sentence (The Tides, $\mathrm{p}$. 284): "There is nothing to tell us whether this theory affords the true explanation of the birth of the moon, and I say that it is only a wild speculation incapable of verification." I leave it to the reader to make his own comments.

\section{The Third-Body Theory.}

In his very interesting little book, "The Birth of Worlds and Systems", published in February, 1911, A. W. Bickerton, Professor of Physics and Chemistry in Canterbury College, of New Zealand, has collected together a series of papers, dating from the year 1878, setting forth a unique speculation, to which he has given the title of "Theory of the Third Body." In order that I may not fail to report him correctly, I shall use his own words quite freely. Fortunately his first chapter is itself an excellent summary of his views :

A pair of dead suns, that is to say two dark stars, colliding, would possess energy sufficient, if suddenly converted into heat to account for the phenomena of temporary stars. All suns possess proper motion. Hence it was evident that the orbits of two suns, in approaching one another, would be similar to that of an ordinary errant comet. There would be mutual deflection and mutual distortion, and it would be extremely improbable that the suns should meet centre to centre. Much more frequently there would be partial impact or grazing collision; therefore, the problem to consider was, what would happen were a pair of dead suns to graze? $* * *$ The portion of each body actually in the path of the other would be torn from the main portion, and these torn-off portions would coalesce into a new or third body, explosively hot and of surpassing brilliancy.

The two diminished suns would pass on, each with a fiery scar where it had been cut. Each would be set rotating, and each would be like a policeman's lantern hung by a string and set spinning. Each would present alternatively its bright and dark face to any point on its equatorial plane. $* * *$ 
Returning to the central body, which the two retreating torn suns were leaving behind between them, one saw that, at the impact, the different elements would be given a temperature that would be proportional to their atomic weight. Oxygen would be 16 times as hot as hydrogen, lead 207 times as hot as hydrogen, each and every one of these elements moving at velocities of hundreds of miles a second, yet all would be tending toward an equality of temperature, as, for example, the hot lead would be robbed of its high temperature by the cooler hydrogen. Then when something like a balance or equality was gained, the energy of unit mass of each element would tend to be inversely as its atomic weight, hydrogen having 4 times the power of escape of helium, 16 times that of oxygen, and 207 times that of lead.

Their velocities would tend to follow the law of Graham, and a kind of atom-sorting would ensue, to which the term "Molecular Selective Escape" was applied. This atom-sorting tells us that the new-born star would soon consist of a brilliant nucleus of heavy elements, surrounded with a set of ensphering shells of different gases; the lightest, hydrogen, being on the outside. $* * *$

The dense nucleus would be rotating, hence the outward rush would not finish with the particles coming to rest; the motion would end in a curve, and all that mass of heavy elements would form a revolving meteoric swarm, which, if the colliding bodies were small, would be a comet. If, on the other hand, it were very large, the swarm might develop into a star cluster, which in turn might become a sun surrounded with countless satellites, a nebulous star. Soon after impact the swarms might become entangled with the variable stars, and might produce the nebulosity at minimum, so characteristic of these bodies $* * *$. This new third body would exercise a retarding influence on the two escaping torn suns, and ought often to wed them into stars. $* * *$

In studying the depths of graze of colliding suns, it was found that when the graze was greater than a third of the whole mass, a kind of whirling coalescence must ensue. Such an event was thought to have given rise to our own Solar System. In this view the planets were pre-existing bodies revolving in any azimuths about one or both of the original colliding suns. These were swung into a plane by the whirl following upon the impact. The moons were pieces of cosmic dust captured by the planets when rarer than they are at present $* * *$.

There is a tendency for the light elements to be expelled from old systems by the high speed to atoms. These tend to congregate in positions of high potential, where matter is sparse. Agencies were found that elevated dissipating energy, and others that tended to disperse matter, until a complete mechanism disclosed itself. That rendered it possible that we exist in a cyclic scheme of creation, in which there is no evidence of a beginning 
or promise of an end, but a cosmic whole infinite and immortal **.

Long ago [p. 2I] both Ritter and myself, by different modes of treatment and different modes of statement of results, showed that in a complete collision of similar gaseous suns, the new sun would be only expanded to one-fourth the density; that is to say, the diameter of the new sun would be the sum of the two diameters of the two similar colliding gaseous suns. I also worked out the interesting result that all the colliding energy was exactly turned into potential energy of expansion, in this way leaving the new sun in possession of the same temperature as the old pair. Moreover, the condition was one of gaseous equifibrium and hence stable $* * *$.

Then [p. 96] what possibly is the right explanation (of the solar system) occurred to me, a suggestion that improves as more and more study is bestowed upon it. It is a kind of combination theory. The planets were captured by the revolving nebula, but they were independent bodies revolving in any azimuth, about one or both of the original bodies whose impact produced the revolving solar nebula. Perhaps the four inner dense planets belonged to one original body, and the four outer rarer ones belonged to the other. Further study showed that all might have belonged to only one of the original bodies. We have had much talk over it, and opinions are still divided $* * *$.

This, then, is the state of our idea of the origin of the Solar System, as far as we have got at present. We have not appreciably altered the idea for thirty years $* * *$.

\section{Criticism of the Theory of the Third Body}

Given a universal affinity of cosmic bodies for eách other, their collision should seemingly be the rule rather than the exception. Indeed, to account for their remaining aloof from each other instead of long ago accumulating in a single heap at the center of things has been one of the chief concerns of Newton and Newtonians. One way which astronomers have adopted to escape this logical difficulty is by the invention of the idea of so-called "proper motions" for stars, on the implied assumption that in the capriciousness, randomness and diversity of such motions lies the desired life-saving principle. In other words, they seek in lawlessness the foil to law. To clinch the matter, they postulate such arbitrary "inherent" motions as being likewise irreducible and self-perpetuating. A second way of escape which they have 
adopted is by assuming that bodies thus mutually attracted cannot fall to each other, but must arbitrarily rotate around their common center of gravity.

Now, we cannot allow Professor Bickerton to blow hot and cold at once; he must in duty bound stick to the precepts he ostensibly professes, or frankly disavow them. That he believes in the reality of proper motions as arbitrary entities he explicitly states, and I think we have no reason to infer aught else than that he regards these conventionally as both inherent and random. In other words, his postulated collisions are not to be construed as arising from the mating stars seeking each other out by virtue of their gravitational affinity, but purely and simply by blind chance. With this important consideration ever in mind, let us weigh the probabilities of such a collision coming to pass in our immediate zone of the heavens - of which alone do we know enough to speak with reasonable confidence.

The star nearest to our sun is Alpha Centauri, and it is 26,000,000 million miles distant; the next nearest is almost twice as far away. Now, by premiss, both Alpha and the sun, in the absence of the ascertained facts, might be moving haphazardly in any direction whatsoever, toward, or away, or parallel, with reference to each other. We will, however, prescribe such a movement on the part of both that shall make it possible for them to collide. Imagine, then, a straight line joining the two, and exactly at the middle thereof, and at right angles therewith, a circular diaphragm having a diameter exactly equal to said line of joinder. We will now stipulate, to begin with, that both Alpha and the sun shall possess proper motions which will cause them to impinge simultaneously on the diaphragm, but we will leave to chance just where each shall pierce it.

By thus conditioning the sun to strike within the area of the diaphragm, we have arbitrarily restricted him to a little less than one-seventh of his original liberty of movement, and so, too, with the star. By restricting both, however, at the same time we enhance the chances 
of collision, not by the sum, but by the product of these amounts, whence the probability against our premiss is, say, fifty to one.

We will now suppose both the star and sun to possess diameters of a million miles (to make computation easy), whence it would follow that (excluding the factor of gravitation) they would be able to pass each other without touching through a square or circular opening of $4,000,000,000,000$ square-miles. Obviously, the chance of either sun or star striking a particular spot on our diaphragm would be in the like ratio as the area of the spot bears to that of the diaphragm or as 2,000 , $000^{2}$ is to $26,000,000,000,000^{2}$, i. e. as 1 to 169,000 , $000,000,000$. But not only this-the sun and star must both strike the same spot and they must do so at the same instant of time, consequently we are obliged to square the terms of our ratio a second time, the arithmetical result of which, for the sake of emphasis, I will write at length, at the same time multiplying it by 50 for the reasons before given. The chances, then, of the sun and this particular star, by far the nearest of all, meeting and colliding is only 1 in $1,428,050,000,000,000,000,000,000$, 000,000 , and even this is an underestimate.

At this juncture it is possible that Professor Bickerton or his supporters may seek to fall back upon the aid of gravitational attraction to bring the stars into touch. To this position I offer three objections, namely; (1) It is contrary to the traditions of the science, traditions to which Mr. Bickerton, along with Newtonians in general, presumably acknowledges fealty, to the effect that unless the approaching star is by its proper motion headed straight at the sun, it cannot strike the latter, but instead must whirl round it in a conic-section orbit. (2) For the star to come fortuitously within even Neptune's distance of the sun, the chances would still be found only one in many quadrillions; altogether too remote a possibility to support Mr. Bickerton's claim to this theory being a "general order or plan of Nature." Lastly, if, in very truth, the star and sun should collide, not because of their 
inherent motions, but on account of their mutual attraction, the energy of their resulting critical velocities, directed, by Newton's second law, toward their respective centers, would bear to the energy of their proper-motion velocities (based on the known value of the sun's) a ratio of something like $500^{2}$ to $12^{2}$, or 1,600 to 1 , thus ensuring total and not partial impact, thereby completely invalidating Professor Bickerton's hypothesis in its very essence.

\section{The Planetesimal Hypothesis}

There are two hinges to the cosmological shutterastronomy and geology. In order to make it swing easily both ways, it is not only necessary that the facts of astronomy be successfully correlated with one another, and the facts of geology with one another, but both of these sets of facts must likewise be mutually coördinated. Nearly a score of years ago Doctor Thomas Chrowder Chamberlin, who for the past quarter-century has been dean of the scientific faculty of the University of Chicago, undertook to construct a system of cosmology from $\boldsymbol{x}$ geologist's point of view. His researches along this line have been summet $\mathrm{p}$ in his recent work, The Origin of the Earth; and the name which he gives his theory is, The Planetesimal Hypothesis.

Now, there are two fundamental astronomical conditions that especially concern geologists which must positively be supplied by any cosmological theory before it can prove acceptable, and both of these concern the sun. These conditions are, first, that a source of heat be found capable of maintaining him at practically an even average temperature over a period of the order of a thousand million years, and, second, that the source so found shall consistently explain the alternation of ice- and genial ages which have occurred in the course of the earth's geological history.

On the first of these problems Doctor Chamberlin does not, nor does he try to, throw any new light, but his chief aim is avowedly to explain the alternation of the 
cold and the warm periods. In the pursuit of this object, he informs us, was he led "into the cosmogonic fens and fogs," whence he emerged with this Planetesimal Hypothesis as his quarry.

Like Professor Bickerton, Doctor Chamberlin derives the solar system from the accidental meeting of our ancestral sun with another star; but with this difference, that whereas the former postulates actual collision, the latter contemplates only "approach to within effective tidal range". He pictures the sun as formerly a solitary star, moving under the head of its so-called proper motion, being fortuitously met or overtaken by just another such star as himself. Beginning by degrees to feel the effect of their mutual attraction, the pair quickly accelerated their approach, and in the natural course of events whirled once around their common center of gravity and then escaped from each other along hyperbolic paths. This incident he supposes to have occurred so long ago that the strange star has had sufficient time to lose itself among the other stars near us.

Coincidently with their thus drawing toward each other, our author conceives the visitor as having raised tides upon the sun (which may or may not have originally possessed axial rotation) and thus stimulated the eruptive tendency which observation reveals to be natural with him, and perhaps with stars generally. When the distance between them was still great, the tides, he opines, were necessarily feeble and the eruptive stimulus correspondingly faint; but later on, as the point of perihelion was neared, the tides increased to a high level and the accompanying eruptions became commensurately more terrific in their intensity, causing the sun to shoot out great bolts of viscous matter through the tidal cones located at the opposite ends of his diameter. While these bolts were thus in the act of rising, or hung suspended in space at heights ranging from zero to some three billions of miles (Neptune's orbit), they were drawn forward by the attraction of the passing star. Such matter as for any reason was deflected only slightly in this manner fell 
back to the sun on about the same spot whence it had issued; other matter being drawn forward somewhat more strongly, fell back also, but angularly, giving, as it were, a fillip to the sun, thereby starting or accelerating his axial rotation; while the great bulk of the matter was pulled forward with sufficient force to lift it into orbital paths more or less elliptical. Once given such motion, under Newtonian interpretation, they would retain it permanently, and in the course of time the larger nuclei, which would inevitably have resulted, would aggregate to themselves the minute particles, or "planetesimals."

Doctor Chamberlin recognizes that, in the first instance, all the orbits would probably be very elliptical, but opines that a composite of many of them ( a condition which would eventually be brought about by a multiplicity of collisions) would no doubt prove more nearly circular. Hence it is, he says, that the orbits of the planets are only slightly eccentric, whereas those of the asteroids, which have (because of their smallness) experienced only relatively few collisions, are quite capricious and, as further corroboration, he points to the fact that Mercury and Mars, the smallest among the planets, have the most eccentric orbits of all. Proceeding further, Doctor Chamberlin argues that, besides thus accounting for the circularity of the orbits of the planets, the planetesimal impacts upon the nucleating bodies probably gave rise to the direct rotations, and, furthermore, doubtless generated such retrograde rotations as are known to exist within the system.

Doctor Chamberlin seeks to explain the recurrence of ice ages in the long past, not by variations in the solar temperature, but by the periodicity of terrestrial crustal convulsions. For this he prepares the ground by denying the sufficiency of the impact hypothesis to explain any part of the earth's present internal heat, and, instead, derives this heat partly from the bodily contraction of the planet and partly from radio-activity. He argues, that during periods of quiescence this internal heat accumulates and, finally, having gained sufficient strength, forces 
an outlet for itself, upheaving the crust into mountain ranges and loading the atmosphere with immense quantities of carbonic dioxide. Now, carbon dioxide is an efficient absorber of heat, hence, in the periods when the atmosphere was rich with it-that is to say, during the interims between the mountain-making cataclysms-the earth experienced her genial climates. On the other hand, however, from the moment of the subsiding of these convulsive movements, the carbon dioxide began to be gradually extracted from the atmosphere, by plants to form coals and other carbonaceous deposits, and by precipitation in the rains and subsequent absorption in the rocks; a process which eventually brought about periods of low temperature immediately preceding the upheaval next in order of events. But let me quote from his own words (Origin of the Earth, p. 103, et seq.) :

In the sun, there is a persistent eruptive tendency of great power. At short intervals, great bolts of sun-substance are shot forth at high velocities. This takes place without any obvious outside stimulus; or, if there be such stimulus, it is not declared. Beyond question if suitable strong stimulus from without were brought to bear on the sun, such as the differential attraction of a passing star, it would respond with eruptions of much greater intensity and mass.

It thus appears that from so simple a cause as the differential gravity called into action by the close approach of one massive body to another, there may arise a graded series of eruptions ranging from fractional ejections to profound disruption and dispersion, according to the closeness of approach, the relative masses of the bodies, and their internal state. The ejected parts will pursue such courses as may be imposed on them by the new forces of attraction brought into play by the changing relations of the two bodies, both of which are necessarily in swift curving motion, while one or both are losing mass by disruptive action. **

For an illustrative case, selected to suit our problem, let our sun, in its ancestral state, be the body approached. For its partner in action, let a more massive star be chosen and, for convenience, let it be so dense and inert that its response to the reaction of the sun upon it may be neglected. In addition it will be convenient to speak of the relative changes of position of the two as if the whole motion were made by the passing star. ***

In selecting the closeness of approach, let us observe that only I/745 of the sun's substance was required to form our whole 
planetary system. There are now known to be eight planets, twenty-six satellites, and about eight hundred planetoids; probably the whole number of the latter may ultimately be found to be a thousand or so. The average mass of these minor solar attendants is thus only about $I / 745,000$ of the mass of the sun. The average mass of the planets, neglecting the planetoids and satellites, is about $\mathrm{I} / 6,000$. Even the largest planetary mass is less than a thousandth of the mass of the sun. It was not necessary, therefore, that the sun should give forth even so much as one-tenth of I per cent of its substance to form the largest planet, assuming that the whole material for the planet was ejected from the sun by a single impulse. The requirement for the earth would be about one three-thousandth of I per cent of the sun. It thus appears that the draft on the sun to supply the substance of the planets was very small relatively. This suggests that the passing star, if it had the mass we have chosen, must surely have had such slight stimulating effect as the case required. We assume therefore only a quite distant approach. $* * *$

Let it be assumed that the eruptivity of the sun was of the same order then as now. At present, the sun is almost daily shooting forth gas-bolts of vast dimensions and often at such velocities that they rise many thousands of kilometers above its glowing surface. Conservative computations assign these eruptive ejections velocities occasionally reaching one hundred or two hundred kilometers per second, though the average speed is less. Estimates by observers of high standing assign much higher velocities in certain cases, some of these rising to several hundred kilometers per second; indeed, velocities that surpass the sun's power of control have been announced. ***

It is assumed that, at the time the nebula was formed, the greater eruptions of the sun were concentrated, as now, in two belts not far from the sun's equator. It is inferred that, as the star approached from a distance, its first feeble stimulus led only to moderate ejectments of sun-substance and that these suffered so slight deviations by reason of the forward pull of the star that they did not escape striking the sun's disk on their return and so carried into the sun a little momentum acquired from the star. This momentum neutralized an equivalent amount of the momentum of the sun's rotation, then opposite to its present rotation. With nearer approach of the star, the eruptions increased in mass and vigor with increased effect on the sun's rotation. With still nearer approach, a portion of the projectiles failed to strike the sun's disk on returning and swung into orbits about it. Later, a still larger part of the increasingly vigorous projectiles passed into orbits, and these orbits grew broader, but certain portions of the projectiles continued to return to the sun and affect its rotation. 
During all this time the pull of the star was oblique to the normal ascensive lines of the sun's greater eruptions, and the sun and star worked at cross-purposes; but, as the star curved into the critical part of its path, where it made its closest approach, it passed directly over the belt of the sun's most effective eruptions, and not only the most favorable co-operation of sun and star were realized, but nearly the maximum mutual attraction. It is assumed that the greatest eruptive bolts were then shot forth, and that they were projected with the greatest velocity. It is taken for granted that the stimulus of vigorous action on the side toward the star would react as stimulus to eruption on the other side, and that nearly simultaneous bolts would issue from the proximate-and from the distal side of the sun. It is supposed that the action would be most effective when the first eruptive belt was crossed, for then the projectile forces drew on the fullest stores of eruptive potency in the sun. The second pair of great eruptions are assigned to the stage when the second belt of solar eruptions, on the farther side of the solar equator, was crossed. These two pairs of eruptive projectiles of the first order are assumed to have been the parents of the four great planets, the two outermost-with the peculiarities of the firstborn- growing later into Neptune and Uranus; the two following, favored by the pulsations set up by the previous great eruptions and by greater facilities for growth, but lacking the fullness of eruptive resources that favored the first pair, consituted the knots that grew into Saturn and Jupiter.

\section{Criticism of the Planetesimal Hypothesis}

If Newton's theory of tidal production is objectively false, as Darwin, Young, Kelvin and a host of others who have made a physical investigation of the tides reluctantly report it to be, and as I think I have deductively demonstrated it to be, of course the Planetesimal hypothesis is false also, and it would seem that nothing more need be said. The tenacity of inherited opinions, however, is unfortunately often far more powerful in molding human opinion than truth itself, and so it becomes necessary to subject the theory in question to a test by the standards generally recognized, whether those standards be, in fact, genuine or spurious.

In a former place it was pointed out that, according to Newton's Corollary, the height of the tide is a function of the thickness of the equatorial ring, and that where no 
such ring exists there can be no tide; hence the sun could, technically, bear no tides, however favorable to their creation the physical circumstances might appear to be, for the simple reason that he has no equatorial protuberance. The learned Doctor could, indeed, reply that the ancestral sun might, for all that, have had such a protuberance-but then, again, it might not.

The next question to settle is as to which was the vera causa of this planet-creating convulsion that Doctor Chamberlin describes-was it the tidal perturbation or the internal disrupting force? The Doctor himself does not make it at all clear which is which, inasmuch as he depicts the duration and progress of the process, not in accordance with the well-known spasmodic, violent, and lawless peculiarities of explosive action, but with the smooth curve of tidal flow and ebb. Although, quantitatively, the tidal force could not have amounted to more than one-millionth of the strength of the eruptive energy requisite to project the planets, all at one time, into their respective orbits, yet he sets up the lesser agent as the controller for the methodical liberation of the greater! Granting that the tidal agitation supplied exactly the stimulus requisite to call the alleged eruptive forces into action, then the latter should have broken loose, not in the modulated manner of a tidal swell, as pictured by our author, but in a sudden and maximal outburst, from which there would follow not gradual intensification of the action, but, rather, gradual subsidence to the normal state of quiescence. In short, he makes the tail wag the dog.

Next, let us compare these two dynamical agencies quantitatively: To project all the planets into their respective orbits would obviously demand the expenditure of precisely as much thermal (explosive) energy as would be generated by their fall thence into the sun. It so happens that this has already been computed for us by Sir Robert Ball (Story of the Heavens, p. 520) :

Were Jupiter to fall into the sun enough heat would be thereby produced to scorch the whole solar system, while all the planets together would be capable of producing heat which, if 
properly economized, would supply the radiation of the sun for 45,000 years.

Unfortunately, Doctor Chamberlin is silent as to the magnitude of the visiting star, contenting himself with the vague statement that "it was more massive than our sun" ; nor is he any more definite as to its perihelion remoteness, merely opining that it was "quite distant." It is therefore impossible for us to reduce to definite terms of thermal heat (for the sake of comparison) the tidal force that Doctor Chamberlin may have in mind. Supposing, though, that this amounted to as much as the radiation of the sun for a period of 16 days-a purposely much exaggerated estimate-it would still be only 1-1,000, 000 as great as his required eruptive forces. To get an idea of this disparity, it may be correctly represented by that of a small boy in a skiff attempting to tow one of our largest battleships.

That a tidal force relatively so insignificant could, by coming fortuitously to the aid of a pent-up force so incomparably greater, not only suffice to release it, but also to master and govern its methodical disbursement and application to cosmic purposes, is manifestly absurd, unless, indeed, there be shown some automatic mechanism or some purposeful intelligence directing to that end. Supposing, on the other hand, the actuality of such an enormous explosive reservoir residing in the sun, how are we to explain either its conservation and accumulation or its multi-millenially deferred deliverance? The incessant ebullition going on in the plastic body of the sun proclaims, not a storing-up of heat but its steady escape and dissipation. How, in the name of sanity, can a tide, however powerful, be postulated as liberating gases already freely escaping of themselves?

It is possible that, in deference to the recent philosophical revulsion against the thermodynamic doctrine of the impermanency of nature, Doctor Chamberlin may imagine his ancestral sun as having entered upon a decline whence it was rescued and resuscitated by this tidal elixir. In that case, one might suppose the sun to have 
acquired a solid crust, of uncertain thickness, capable of penning up the gathering tempests within. The query then arises as to just how thick that crust should have been in order to meet the requirements of the situation.

One way to dispose of this query would be to point out that the very existence of such a crust, thick or thin, being, by premiss, the result of cooling off, precludes the hypothesizing of any such rampant interior forces at all, and that, once begun, the cooling process would necessarily continue progressively to a state of total frigidity and inaction. To assert that such a decadent sun harbored a thermal reserve equal to 45,000 years' solar radiation, yet allowed its exterior to chill into an imprisoning shell, is a contradiction in terms. Furthermore, the stauncher the shell the stronger the tide required to breach it, supposing such an event to have really occurred; and this, as we shall see, raises up new difficulties.

Whether through inability or negligently, Doctor Chamberlin discreetly refrains from citing a single concrete illustration of what relation, if any, between the star's mass and its solar distance would have sufficed to meet the demands of his problem; subtly leaving upon the mind of the uncritical reader the impression that the possible choice of combinations of these two factors is practically unlimited. Let us look into this matter closely :

In the first place, we must remember that the planets do not revolve around the sun with equal velocities, but with velocities varying greatly with their central distances. Thus Mercury, the nearest planet to the sun, travels some eight times faster than Neptune, the outermost. Doctor Chamberlin assumes that all the planets alike simultaneously acquired their tangential motions from the gravitational attraction of the passing star. If this were so, why did they not all start out with practically the same speed? In order to make clear how the planets acquired their Keplerian velocities, Doctor Chamberlin ought to show us just the spot where the 
strange star might have been with reference to the string of planets, just how large it might have been, and just how far from the sun, in order to bring about the present planetary scheme. Had our author succeeded in figuring out any such combination, he would doubtless have mentioned the fact in his book; but he doesn't. Nor shall I consume the reader's time by mathematically demonstrating the impossibility of any such combination existing. This much, at least, should be plain, that inasmuch as Mercury travels faster than any other planet, the star would have to be predicated as quite close to the sun and certainly not as far away as Venus, else the latter should possess the higher velocity; which it doesn't.

Inasmuch as only terrestrial tides have come within our daily experience, the question has never specifically arisen as to what effect, if any, the mass of the body affected has on the heights of its tides. For example, suppose the sun were an exact duplicate of the earth, save in the one respect of mass, and that the two were equi-distant from each other and from the moon, would the lunar tides on the sun be then exactly as high as those on the earth, or would they be directly proportional to their mass, or would they be inversely proportional, or just what rule would obtain? In the absence of any surer guide, let us assume the tidal heights to be, inversely, functions of the masses of the bodies affected, and that the star, in order to raise upon the sun tides commensurate with our terrestrial tides, had to exceed the size of the sun as much as the latter outweighs the earth. Supposing, then, the star to have come to within $46,000,000$ miles of the sun (i. e. one-half the earth's mean distance, and one-third way between Mercury and Venus) it must, under Newton's rule of cubes, have possessed a mass some 40,000 times the solar mass and a diameter of $18,000,000$ miles! It goes without saying that a body of such immensity would have swallowed up our pygmy sun in short order, nor left a vestige behind. Moving the star out farther does not mend matters ; if to the earth's distance, its diameter would have to be increased to $36,000,000$ 
miles, and if to Neptune's, more than a billion. All this calculation is based on the exaggerated supposition that a tide upon the sun comparable to that the latter produces upon the earth would have sufficed to pry open a crust which, until then, had been able to resist the incessant straining of a jinnee a million times stronger. That Doctor Chamberlin himself is thinking of a comparatively weak tide sufficiently appears from his mild expressions: "For its partner in action let a more massive star be chosen" * * "only a quite distant approach," and "let it be so dense and inert that its response to the reaction of the sun upon it may be neglected."

In one of his veiled allusions to Professor Bickerton's theory, our author seeks to emphasize the greater probability of a "near approach" over actual collision, in which contention he is clearly justified. But, though the argument against the probabilities of his own hypothesis is thereby relatively weakened, it still remains strong enough to overthrow his, too. In order to facilitate the calculation, let us assume for the maximum field of " $\mathrm{ef}$ fective approach" a diameter of 200,000,000 miles, then the area of our original postulated diaphragm would be to it in the ratio of $200^{2}$ to $26,000,000^{2}$, or as 1 to 16,900 , 000,000 , which latter number being squared and then multiplied by 50, as previously explained in the discussion of Professor Bickerton's hypothesis, yields the probabilities against even such an approach as 14,265,500,000,000,000, 000,000 , to one!

Next to the supreme problem of the origin, maintenance, and regulation of the planetary motions, comes that of the source of the solar heat. Heretofore it has been the aim of cosmologists to seek a solution of both these problems concurrently and compatibly, but Doctor Chamberlin has unique ideas of his own. Though ostensibly professing to believe in the classical division of energy into potential and kinetic, and that old stars are thermally impoverished and moribund, he naïvely makes our ancestral and presumably senile sun overturn the order of things and hurl the planets into their distant 
places. He has just reversed the film. Having started out with the avowed object of clearing up the mystery of climatic revulsions, he has ended by only floundering deeper into the bog out of which he volunteered to lead us.

\section{The Theory of Arrhenius}

The celebrated Director of the Physico-Chemical Nobel Institute of Stockholm, Svante Arrhenius, has expounded his views on this subject of cosmology in a small volume called Worlds in the Making. As Doctor Chamberlin's theory was conceived from the viewpoint of a geologist, so is this one conceived from the viewpoint of the astrophysicist and chemist.

According to a recent doctrine, the molecules of matter are believed to be in constant motion. Usually these are so closely associated that they are much subject to collision, but in free space, where they seldom strike against one another, they are supposed to travel in straight lines at velocities varying with their kinds. Such motions when less than the "parabolic velocity" do not permit the escape of the given molecule, but when the contrary is the case, the molecule, it is declared, will never again return to the cosmic body from which it took its flight. Building upon this uncertain base, Arrhenius imagines these vagabond molecules to accumulate fortuitously in the dust nebulæ which, as I have previously explained, modern scientists assert to be borne out by light and electrical repulsive forces.

As the molecules of matter are fundamentally construed to be perfectly elastic, Arrhenius makes no constructive use of their high velocities, but the dust particles he supposes to beat each other into a glow and in this state to attach to themselves the various free molecules with which they come in contact. Thus, he says, are the comets and the nuclei of planets formed.

The orbital motions of the planets he "explains" thus (ibid., pp. 203, 204): 
By their collisions with the masses of gases which they encounter, they (the dust particles) gradually assume a circular movement about the axis of rotation of the nebula. In this rotation they condense portions of the gases on their surface, and hence acquire a high temperature-which they soon lose again, however, owing to the comparatively rapid radiation.

Our considerations lead to the conclusion that there is rotating about the central body of the nebula an immense mass of gas, and that, outside this mass, there are other centres of condensation moving about the central body together with the masses of gas concentrated about them. Owing to the friction between the immigrated masses and the original mass of gas which circulated in the equatorial plane of the central body, all these masses will keep near the equatorial plane, which will therefore deviate little from the ecliptic. We thus obtain a proper planetary system, in which the planets are surrounded by colossal spheres of gas like the stars in the Pleiades. If now, the planets have very small mass by comparison with the central body-as in our solar system-they will be cooled at an infinitely faster rate than the sun. The gaseous masses will soon shrink, and the periods of rotation will be shortened; but for those planets, at least, which are situated near the centre, these periogds will originally differ little from the rotation of the central body. The dimensions of the central body will always be very large, and the planets circulating about it will produce very strong tidal effects in its mass. Its period of rotation will be shortened, while the orbital rotation of the planets will tend to become lengthened. Thus the equilibrium is disturbed; it is re-established again, because the planet is, so to say, lifted away from the sun, as G. H. Darwin has so ingeniously shown with regard to the moon and the earth. Similar relations will prevail in the neighborhood of those planets which will thus become provided with moons. Hence we understand the peculiar fact that all the planets move almost in the same plane, the so-called ecliptic, and in approximately circular orbits; that they all move in the same direction, and that they have the same direction of rotation in common with their moons and with the central body, the sun. It is only the outermost planets, like Uranus and Neptune, in whose cases the tidal effects were not of much consequence, that form exceptions to this rule.

Our author's second aim is to point out what he regards as Nature's method of self-restoration by reversing, as it were, the thermal hour glass and starting the heat current on the downward course once more. Quoting Clausius' old maxim, "The energy of the universe is 
constant; the entropy of the universe tends to a maximum"', he proceeds :

The famous Scotch physicist, Clerk Maxwell, has conceived of this case. Imagine a vessel which is divided by a partition into two halves, both charged with a gas of perfectly uniform temperature. Let the partition be provided with a number of small holes which would not allow more than one gas molecule to pass at a time. In each hole Maxwell places a small, intelligent being (one of his "demons"), which directs all the molecules which enter into the hole, and which have a greater velocity than the mean velocity of all the molecules, to the one side, and which sends to the other side all the molecules of a smaller velocity than the average. All the undesirable molecules the demon bars by means of a little flap. In this way all the molecules of a velocity greater than the average may be collected in the one compartment, and all the molecules of a lesser velocity in the other compartment. In other words, heat-for heat consists of the movements of molecules-will pass from the one constantly cooling side to the other, which is constantly raising its temperature, and which must therefore become warmer than the former.

In this instance heat would therefore pass from a colder to a warmer body, and the entropy would diminish.

Nature, of course, does not know any such intelligent beings. Nevertheless, similar conditions may occur in celestial bodies in the gaseous state. When the molecules of gas in the atmosphere of a celestial body have a sufficient velocity-which in the case of the earth would be $1 \mathrm{rkm}$. ( 7 miles) per second-and when they travel outward into the most extreme strata, they may pass from the range of attraction out into infinite space, after the manner of a comet, which, if endowed with sufficient velocity when near the sun, must escape from the solar system. According to Dr. Johnstone Stoney, it is in this way that the moon has lost its original atmosphere. This loss of gas is certainly imperceptible in the case of our sun and of large planets like the earth. But it may play an important part in the household of the nebulæ, where all the radiation from the hot celestial bodies is stored up, and where, owing to the enormous distances, the restraining force of gravity is exceedingly feeble. Thus the nebulæ will lose their most rapid molecules from their outer portions, and they will therefore be cooling in these outer strata. This loss of heat is compensated by the radiation from the stars. If, now, there were only nebulæ of one kind in the whole universe, those escaped molecules would finally land on some other nebula, heat equilibrium would thus be established between the different nebulæ, and the "heat-death" be realized. But we have already remarked that the nebulæ enclose many immigrated celestial bodies, which 
are able to condense the gases from their neighborhood, and which thereby assume a higher temperature.

The third aim of Doctor Arrhenius is to account for the sun's heat. To this end he prepares the ground by exposing the shortcomings of Helmholtz's hypothesis, as well as of others, and then selects for his own the now familiar conception of the atomic dissociation of matter. In this particular field he is esteemed preëminent, having won the Nobel prize in 1903 for his researches along this line. To quote again from his book (p. 91):

It is quite incorrect to assert that high temperatures must necessarily decompose all chemical compounds into their elements. The mechanical theory of heat teaches us only that at rising temperatures products are formed whose formation goes hand in hand with an absorption of heat. Thus, at a high temperature, ozone is formed from oxygen, although ozone is more complex in composition than oxygen, and by this reaction 750 calories are consumed when one gramme of oxygen is transformed into one gramme of ozone. We likewise know that in the electric arc, at a temperature of about $3000^{\circ}$, a compound is formed under consumption of heat by the oxygen and nitrogen of the atmosphere. A new method for the technical preparation of nitric acid from the nitrogen of the air is based upon this reaction. Again, the well-known compounds, benzene and acetylene, are formed from their elements, carbon and hydrogen, under absorption of heat. All these bodies can only be synthetiżed from their elementary constituents at high temperatures. We further know from experience that the higher the temperature at which a reaction takes place, the greater, in general, the amount of heat which it absorbs.

A similar law applies to the influence of pressure. When the pressure is increased, such processes will be favored as will yield products of a smaller volume. If we imagine that a mass of gas rushes down from a higher stratum of the sun into the depths of the sun's interior, as gases do in sun-spots, complex compounds will be produced by virtue of the increased pressure. This pressure must increase at an immense rate towards the interior of the sun, by about 3500 atmospheres per kilometre. The gasses which dissociate into atoms at the lower pressures and the higher temperatures of the extreme solar strata above the photosphere clouds enter into chemical combination in the depths of the spots, as we learn from spectroscopic examination. Owing to their high temperatures, these compounds absorb enormous quantities of heat in their building up, and these quantities of heat are to those which are concerned in the chemical processes 
of the earth in the same ratio as the temperature of the sun is to that at which the chemical reactions are proceeding on the earth. As these gases penetrate farther into the sun, temperature and pressure are still more and more increased, and there will result products more and more abounding in energy and concentration. We may, therefore, imagine the interior of the sun charged with compounds which, brought to the surface of the sun, would dissociate under an enormous evolution of heat and an enormous increase of volume. These compounds have to be regarded as the most powerful blasting agents, by comparison with which dynamite and gun-cotton would appear like toys. In confirmation of this view, we observe that gases when penetrating into the photosphere clouds are able to eject prominences at a stupendous velocity, attaining several hundred kilometres per second. This velocity surpasses that of the swiftest rifle-bullet about a thousandfold. We may hence ascribe to the explosives which are confined in the interior of the sun energies which must be a million times greater than the energy of our blasting agents. (For the energy increases with the square of the velocity.) And yet these solar blasting agents have already given up a large part of their energy during their passage from the sun's interior. It thus becomes conceivable that the solar energy-instead of holding out for 4000 years, as it would if it depended upon the combustion of a solar sphere made out of carbon-will last for something like four thousand million years. Perhaps we may further extend this period to several billions.

As his final and main thesis, the learned savant undertakes to explain how life-germs are disseminated through the universe. First, he disposes of the argument in behalf of spontaneous generation by citing its want of ocular proof and absence of physical demonstration. He then reminds us of Schwarzschild's deduction, that particles approximating $.00016 \mathrm{~mm}$. in diameter are the most responsive to radiation pressure, and seeks to extend this category to include plant and animal spores and germs. These, he says, being borne away, literally, on the wings of light find lodgment on distant worlds, and originate new chains of evolution more or less resembling life terrestrial.

The theories above outlined, circumscribed, strained, and mutually contradictory as they may appear, repre- 
sent the supremest efforts of modern scholastic astronomy. Their very multiplicity is, of itself, in my judgment, the sufficient proof of their unsatisfactoriness and unsoundness, and demonstrates the urgent need of a cosmic philosophy big enough and broad enough to include the entire physical universe under one clear, consistent, dynamical generalization, and in which practical common sense shall count for more than baseless assumptions and frenzied speculation. 


\section{$\mathrm{X}$}

\section{THE SUN}

\section{$\mathrm{H}$}

OW wonderful the sun!

To the casual observer our two luminaries appear to be of about the same size, but the disparity between their actual sizes is almost incredibly great. In round numbers the sun is 400 times further from us than the moon, consequently their real diameters must bear to each other the same ratio. The volumes of spheres, however, are to each other as the cubes of their diameters; hence the bulk of the larger luminary is some $64,000,000$ times that of the smaller. Imagine, then, the substance of the former to be divided into this number of little moons, strung together like beads upon a strand, how far do you suppose this rope of luminous orbs would reach for decking out our system? For answer, multiply 2160 miles (the lunar diameter) by $64,000,000$ and compare the result, 138,000,000,000 miles, with the following dimensions, at the same time allowing liberally for artistic effects:

Sum of the distances from the sun

Miles

to each of his several planets ........ $6,280,000,000$ Allowance for festooning ........ 2,000,000,000 Sum of the distances from the various planets to each of the others . . . . . 43,000,000,000 Allowance for festooning . . . . . . 12,000,000,000 Sum of all the planetary orbits .....44,000,000,000 For adornment of all the subordinate systems after the same manner ..... 1,000,000,000 Surplus for latticing the asteroids together .................. 33,720,000,000 
Of the intensity of the sun's brightness an idea may be gained by a comparison of the following facts: At the distance of Neptune the sun subtends an angle of slightly more than a minute of arc, or, to cite an illustration, it subtends the same angle as a cent piece does at the distance of 200 feet-obviously too small an area to be recognized as a disc. Now, it has been repeatedly estimated, with care, that we derive 600,000 times as much light from the sun as we do from the full moon; hence, putting two and two together, it follows that the solar spark yields as much light to the inhabitants of Neptune as would a battery of 666 full moons such as our own.

To the novice it might seem a comparatively easy matter to determine the temperature of the sun, but that it is by no means so is shown by the great diversity in the estimates made at various periods by recognized authorities; varying, as they do, between 6,000 and upwards of a million degrees. In his recent work, The Sun (pp. 109116), after describing the four methods which he deems the most dependable, Doetor Abbot says: "Hence we conclude that there is a high probability that the average temperature of the apparent photosphere exceeds $5860^{\circ}$, or even $6260^{\circ}$, on the absolute Centigrade scale, and may be as high as 7,000 absolute Centigrade." In passing let me record my substantial agreement in this estimate.

However, that there is still a mystery behind this one of temperature yet to be explained away, will appear from the following considerations : Construing the earth's disc as part of the surface of a sphere 186,000,000 miles in diameter (i. e., double the earth's distance from the sun), and comparing this surface with that of the sun, whose diameter is 865,000 miles, and, further, remembering that the surfaces of spheres are to each other as the squares of their diameters, it develops that the radiation of each square foot of the sun's area must answer for the heating of each 46,000 square feet of the earth's area, considered as a disc. We should not forget, however, that the sun's rays fall on the same hemisphere only half 
of the 24 hours and that it is a hemisphere, and not a flat disc, with which we have to deal. Making corrections for these facts, we find that, instead of our former result remaining at 46,000 , it must be increased to 184,000 square feet, being equivalent to a small city block 425 feet each way, or, in the farmer's standard, 4- $1 / 4$ acres.

Here we must call to mind that superimposed upon this block is a great column of air, variously estimated at from 50 to 150 miles in height, but attenuating rapidly with the altitude. Not only, however, does the air decrease in density with altitude, but it decreases in temperature as well; and where the column may be supposed to end, there is no confining wall, but the doorway is leff wide open, permitting the free escape of warmth into the boundless outdoors of space, where the reigning temperature is generally believed to be absolute zero, or $-273^{\circ} \mathrm{C}$. Inasmuch as it is impossible to make computations without definite figures to begin with, let us try to approximate the facts, as nearly as we may, by assuming that the atmospheric column in question is equivalent to another column of air of sea-level density 7 miles in height and possessed of an average temperature of $0^{\circ} \mathrm{C}$. It is such a volume as this, then, 184,000 sq. ft. $x 36,000$ feet, that modern scientists suppose to be maintained $273^{\circ} \mathrm{C}$ above the absolute by the unaided radiation from $a$ single square foot of solar surface! We may emphasize these figures by mentally dividing this great column into smaller proportional dimensions. Imagine a hall 100 feet in the clear covering a ground space equivalent to a small city block, then the contents will be 1-360 of our column. Again, let it be assumed that the outside temperature is $0^{\circ} \mathrm{C}$ and that that within the hall is maintained at $27.3^{\circ} \mathrm{C}$ (the main doors being all the time kept wide open) how large, think you, need the miniature sun be to serve as the furnace? The cubical contents of the hall being only 1-360 that of our atmospheric column, and the lift in temperature only 1-10 as high, there would be required a heat source possessing only 1-3600 of a square foot of surface, or 1-5 inch square, indicating a sun no 
larger than a buck-shot! In this estimate, be it noted, we have omitted to take into consideration the oceans, which, according to the teaching of our scientists, are maintained above freezing point solely by the sun's radiation. Were we to allow for this added achievement on the part of the real sun, the miniature one need be scarcely more than half as large as the buck-shot previously arrived at! I earnestly call upon the reader to ponder these wonders seriously in order that he may hereafter the better realize the necessity of discarding, as inadmissible, all solar hypotheses based on the doctrine of conservation of energy, and in order, also, that he may more keenly appreciate the heretofore overlooked role that the ebullition of the sun plays in the intensification of his radiation.

Not less amazing, when we attempt to reconcile it with the doctrine of conservation, is the reckless profligacy of Nature. All the planets taken together do not intercept more than ten times as much of the sun's radiation as the earth does, yet the latter grasps only a paltry $1-2,000,000,000$ of it; the rest being uselessly dissipated in the depths of empty space, and to all appearance lost forever. What human spendthrift ever so lax and abandoned as to squander at the rate of $\$ 200,000,000$ for a single dollar's worth! Nor is the sun the only celestial profligate, for the stars, one and all, vie with him in these excesses. Compare with this state of facts what Sir Robert Ball says (Story of the Heavens, p. 579) to the effect that were the sun composed of the best of coal, and this burned in pure oxygen, it would burn itself out in less than 6,000 years; and that passage from Doctor Newcomb, previously quoted, in which he says, that were the sun merely losing energy like an ordinary hot body cooling off, it would be so cooled off in the course of 3,000 or 4,000 years as no longer to radiate much heat!

There is every reason to believe, from spectrosopy as well as analogy, that the sun is composed of the same materials as the earth, and much in the same proportion; but, strange to relate, the former's density is only one- 
fourth as great, despite the circumstance that his integral attraction is nearly thirty times greater. By the logic of current theories he should have a diameter of scarcely half the size. Why, then, is he so distended? Doctor See has estimated the superincumbent pressure at a depth of one-tenth the sun's radius at 21,000,000 atmospheres, and the pressure at his center should be at least $100,000,000$ atmospheres. What, then, is the physical state of his central substances?

One of the most peculiar things about the sun is the manner of his rotation on his axis. Carrington and Spoerer, noted observers of the sun showed:

1. That the sun rotates around an axis inclined about $7^{\circ}$ to the plane of the ecliptic, and so that the axis points midway between the polar star and Vega to a position in right ascension $18 \mathrm{~h} .44 \mathrm{~m}$. and declination $64^{\circ}$. 25 days.

2. At the solar equator the rotation occurs in about

3. The period of one complete rotation increases on either side of the equator equally, and is about $27-\mathrm{T} / 2$ days at $45^{\circ}$ north or south solar latitude.

As to the high degree of the roundness of the solar dise this quotation from Doctor Abbot will show (The Sun, p. 26) :

Poor has lately maintained that observations indicate that the sun's equatorial and polar diameters vary relatively as much as $0 . I^{\prime \prime}$ during a sun-spot cycle of eleven years. According to him, the equatorial diameter is the larger at sun-spot maximum, and the polar diameter the larger at sun-spot minimum. Ambronn, however, denies that this is supported by the observations, and Moulton opposes so large a variation on theoretical grounds.

That such phenomena as sun-spots exist has been known for more than four centuries. These spots are not always visible, but come and go in cyclical periods, increasing from a state of almost complete absence for (according to Newcomb) 4.62 years and then decreasing for a further space of 6.51 years-making up a total period of 11.13 years. It must be noted, however, that 11.13 years is only the mean interval, the individual 
periods ranging between 7.3 and $\mathbf{1 7 . 1}$ years. Young has described the formation and life history of sun-spots in these words:

There is no regular process for the formation of a spot. Sometimes it is gradual, requiring days or even weeks for its full development, and sometimes a single day suffices. Generally, for some time before the appearance of the spot, there is an evident disturbance of the solar surface, manifested especially by the presence of numerous and brilliant faculae, among which, "pores" or minute black dots are scattered. These enlarge, and between them appear grayish patches, apparently caused by a dark mass lying veiled below a thin layer of luminous filaments. The veil grows gradually thinner, and vanishes, giving us at last the completed spot with its perfect penumbra. The "pores", some of them, coalesce with the principal spot, some disappear, and others constitute the attendant train. When the spot is once completely formed, it assumes usually an approximately circular form, and remains without striking change until its dissolution. As its end approaches, the surrounding photosphere seems to crowd in upon and cover and overwhelm the penumbra. Bridges of light, often many times brighter than the average of the solar surface, push across the umbra, the arrangement of the penumbra filaments becomes confused, and, as Secchi expresses it, the luminous matter of the photosphere seems to tumble pell-mell into the chasm, which disappears and leaves a disturbed surface marked with faculae, which in their turn subside after a time. As intimated before, however, the disturbance is not unfrequently renewed at the same point after a few days, and a fresh spot appears just where the old one was overwhelmed.

The spots usually appear not singly, but in groups-at least, isolated spots of any size are less common than groups. Very often a large spot is followed upon the eastern side by a train of smaller ones; many of which, in such a case, are apt to be very imperfect in structure, sometimes showing no umbra at all, often having a penumbra only upon one side, and usually irregular in form. It is noticeable, also, that in such cases, when any considerable change of form or structure shows itself in the principal spot of a group, it seems to rush forward (westward) upon the solar surface, leaving its attendants trailing behind. When a large spot divides into two or more, as often happens, the parts usually seem to repel each other and fly asunder with great velocity-great, that is, if reckoned in miles per hour, though, of course, to a telescopic observer the motion is very slow, since one can only barely see upon the sun's surface a change of place amounting to two hundred miles, even with a very high magnifying power. Velocities of three or four hundred miles an hour are 
usual, and velocities of one thousand miles, and even more, are by no means exceptional.

The average life of a sun-spot may be taken as two or three months; the longest yet on record is that of a spot observed in I840 and I84I, which lasted eighteen months. There are cases, however, where the disappearance of a spot is very soon followed by the appearance of another at the same point, and sometimes this alternate disappearance and reappearance is several times repeated. While some spots are thus long-lived, others, however, endure only for a day or two, and sometimes only for a few hours.

As to the causes of sun-spots Doctor Abbot says (The Sun, p. 188):

The causes which produce sun-spots, being as yet doubtful, or perhaps it is better to say entirely unknown, the causes of their periodicity and of the irregularity of the periods are, of course, also unknown. Attempts have been made to connect the period with the times of revolution of the planets, and, indeed, the mean length of the sun-spot period is not far from the period of the revolution of Jupiter (II.86 years). No satisfactory case for a connection between these phenomena is yet made out.

During his total eclipses the sun is seen to be surrounded by a pale luminous veil, called the corona, which during sun-spot periods is fairly regular all around, but in the quiescent periods is confined to his equatorial regions. It has been remarked of the corona, as matters requiring explanation; first, that it appears to partake of the uniformity of the sun's rotation, and, second, that comets which have been observed to pass through it suffered no retardation.

The chromosphere is a layer of incandescent gases having a distinctly reddish tint, and comes next below the corona; but whereas the latter is never observable except during total eclipses, the former can be seen at any time with the aid of telescope and spectroscope combined. Hydrogen is its chief component.

The so-called prominences or protuberances are upward projections in the chromosphere, but exist only temporarily. They are of two kinds, eruptive, which arise from some violent explosive action, and quiescent, which assume graceful tree-like forms and seem rather to evolve than spring. 
Next beneath the chromosphere comes the reversing layer, relatively dark, of uncertain depth, and containing the cooler gases which are responsible for the Fraunhofer lines in the solar spectrum.

And, finally, the shell or layer that will engage our attention here most, and which holds within it the key to them all-the photosphere.

We see, then, from the premises, that the sun presents not one only but a complexity of enigmas, all of which must be solved consistently with each other. His longevity; his occasional lapses and recoveries; the intensity of his light and heat; his density; his eruptive character; his sun-spots; their periodicity; his corona; its unresistingness; his absence of oblateness; his axial rotation per se; the cause of his equatorial acceleration; his share in the genesis of his system; his beginning and his destiny; his dynamical relationship to the stars in general; his relationship to comets and meteors; his path in space; the source of his magnetism; his connection with such terrestrial phenomena as earthquakes, electrical storms, climatic reversals, and the like-all these we shall treat of in detail and prove them intimately and causally interdependent.

\section{SOLUTION OF THE SOLAR PROBLEMS}

One of the many serious objections to the Nebular Hypothesis is the fact that the residual mass, to wit, the sun, does not revolve on its axis with anything like the velocity demanded of it. According to Kepler's third law, this velocity should be at least 200 times more rapid than it is in nature. Furthermore, according to Laplace's idea, the entire solar body should revolve as a rigid solid, notwithstanding the mobility of its component materials; whereas there is noted a decided acceleration of the equatorial regions, amounting to as much as ten per centum over what it is in the middle latitudes. Attempts have been made by both Chamberlin and See to account for this phenomenon; the former by supposing that his ancestral sun may have had a different direction 
of axial rotation prior to its encounter with the strange star, and that both this old and the new rotation imposed by the encounter persist together; while the latter attributes the phenomenon to the infall of meteoric matter.

The explanation that I submit is this: Under the principle of equilibrium as hereinbefore expounded, the sun is the king-pin of the self-balancing, rotating machine known as the solar system. As a member of that system, he is obliged to aid in this balancing process to the extent imposed upon him by Nature. Between him and the planets, however, there is this fundamental distinction, namely, that whereas the planets have but one way open to them-orbital movement-whereby they can comply with the equilibristic law, the sun, being in the very center and astride the fulcrum, has two ways, to wit; first, by axial rotation and, secondly, by oscillating or "see-sawing" to and fro across the fulcral point. Now, we all know that, however complex and multifarious her operations, Nature never forgets herself or becomes confused, but carries all her processes along automatically and concurrently in perfect harmony. Accordingly, the sun does not choose either of the balancing methods open to him to the total exclusion of the other, but pursues both of them consistently. Is it not obvious, then, that, on this theory, the equatorial rim of the sun being farthest from the center should partake more of the orbital nature of movement, while the more central parts, being closer to the fulcrum, should seek the more direct course of shuttling? Of course, were the sun a rigid solid, he would necessarily rotate as a whole; but he is far from answering this description, being, on the contrary, fluid throughout, so that all his parts are measurably free to follow their respective equilibristic impulses.

Pursuing this train of reasoning further, let us conceive the sun severed in half on the line of his equator and then study his internal evolutions, fixing our attention now, not on his structure, which will be specially treated later, but on the torsional stresses that intestinally afflict him. To aid in clarifying the explanation, 
let us assume the section thus presented to view to consist, from center to circumference, of many concentric layers; then would all of these layers possess some velocity of rotation about the sun's axis, and in the same sense. However, not one of these would rotate uniformly with its neighbor on either side, but each would travel a shade faster than the adjacent inner layer and a little slower than the adjacent outer layer; producing by this process an enormous amount of friction. It is to this friction that I attribute the electric and magnetic qualities of the sun.

While thus we have the solar interior exposed to view, let me utilize the occasion by pointing out several other peculiarities in the sun's structure. One of these concerns the distribution of his density. Granting that the chemical constitution of the sun, qualitatively and proportionally, is identically the same as the earth's (as we have good grounds for assuming) and bearing in mind that the advantage of self-compressional power lies overwhelmingly in his favor, why is it that his density instead of being decidedly greater than that of our earth is only a quarter of hers? It is all well enough to say that the difference is due to the patent fact that the sun is vastly hotter than our planet, that the application of heat alters the state of substances from solid to liquid and thence from liquid to vapor, or gas, and that in each of these several states the volume of the given substance is greatly altered; but all this is only empirical, and not enough. Though solids and liquids do indeed expand and contract with heat, they do so only within very narrow limits; but gases derived from them seem to possess incredible expansive capabilities. Clearly, the distension of the sun cannot be accounted for on the score of expanded solids or liquids as such, but must seek its explanation solely in the buoyancy of gases. But here we meet with another difficulty; the sun, though very much lighter, bulk for bulk, than the kernel of the earth, is still 1.4 times heavier than water; how, then, could the gases exist as gases in a space less than their own liquids would 
require? Besides, who can guarantee that a ball of gas the size of the sun, or for that matter a gaseous ball of any size, unconfined, will preserve its identity and remain intact and globular?

Doctor Abbot assumes that the sun is entirely gaseous, but refrains from discussing the manifest objections to that assumption. It is a fact known and commented on by every solar observer, that at least the larger sun-spots preserve their identity, and even their outline, for weeks and sometimes months on end, and it is authentically recorded that the great spot of 1840-1841 lasted as much as eighteen months. How Doctor Abbot and other Doubting Thomases, beholding these wounds in the sun's side, can perversely persist in declaring him not matter, but matter's ghost, is beyond my fathoming.

Many scientists-probably the majority of themappear to believe in a fourth state of matter, as some mathematicians do in a fourth dimension. The reason for this hypothesis lies in their endeavor to specify an effect for the enormous pressures brought to bear on the central parts of such immense bodies as the earth, Jupiter and the sun. Some have gone so far as to invent a description of this supposititious state-just as they dogmatize about the nature of the ether-and picture it as of a "waxy" consistency. A little reflection ought to convince these philosophers, not only of the unlikelihood of such an anomalous state of matter existing, but also of its undesirability from the standpoint of theory. The mystery here demanding solution is the sun's lack of density, and this mystery is only rendered altogether impenetrable by imagining his figure packed solidly to the core.

Mature deliberation on the problem of the solar density can lead to but one satisfying conclusion, a conclusion, too, that leads us by a well-blazed path to the clearing-house of practically all the present enigmas of the sun and stars.

To begin with, physicists have demonstrated by practical experiments that there is what they call a 
critical point in gases; signifying that, given a certain temperature (varying, of course, with the substance), no application of pressure, however great, that may be brought to bear will avail to reduce the gas to the form of a solid or liquid. Now, if this generalization is valid, then it necessarily follows that the heart of the sun is gaseous, since there the temperatures prevailing are demonstrably above the critical point of every known chemical element. But though the interior of the sun is gaseous, it does not follow that the whole globe is. On the contrary, when we consider his high internal temperatures, and his high density as compared with what he ought to possess were he in a solely gaseous state, we soon come to realize that the sun can be nothing else than an enormous inflated solid or liquid shell. Not only does this conclusion obviate the postulation of a chimerical fourth state of matter, and account for the persistence of sun-spot identity and the enigmatic low density of the sun, stars and planets, but, as we shall presently see, it also supplies the clue to practically every mystery of star-life and star-behavior from the moment the star crystallizes out of the nebular dust until it dissolves into nebular dust again.

After all, what is this doctrine of the critical point except a general recognition of the explosibility of all substances by percussion, or crushing: Dynamite may be detonated by raising its temperature, by a sudden blow, by friction, or by strong pressure. So may any and every other substance. "Give me," said Archimedes, "a lever long enough and I will move the earth", and so I say, give me a press powerful enough and I will detonate any known substance, whatever be its temperature, from gunpowder to granite. Apropos of this statement let me quote from the pen of that world-wide authority, J. W. Gregory, Professor of Geology at the University of Glasgow (Geology of To-day, p. 157):

The ordinary rocks on the earth's surface are crushed into powder when subject to a weight of somewhere between two and thirty tons to the square inch. Hence if a column of ordinary rocks were built from 3 to 5 miles high, the base would be crushed 
by the weight of the upper part. Rocks are often found in such a condition of strain that they fly to pieces when exposed on one side in a deep mine. In some mining fields the rocks cut through in driving a mine tunnel suddenly explode owing to the strain of the overlying weight, just as a spring may snap when overloaded. Fragments are thrown from the rock face in 'rock blasts', and have caused many fatal accidents.

This detonation of rocks (and other materials as well, including metals) is no more than should be philosophically expected. That the three states of matter, solid, liquid and gaseous, must follow each other in a cycle at peril of destroying Nature's very life seems all but axiomatic. Were the fact otherwise, matter, once caught in the trap of gravitation and held down by superincumbent layers, would become perpetually staled and nullified; which is repugnant to sound reason. We know from experience that the heavier the pressure brought to bear, the less space does the compressed substance occupy. Were this rule to apply indefinitely, it would mean nothing less than the final extinction of matter by shrinkage into no space at all. Where does this trend cease and the recovery begin? How does Nature restore the balance? What logical thing remains for the tortured molecules to do, but to rebel and bombard the walls of their prison till these yield as to an acid and vouchsafe escape?

Heretofore it has always been assumed as quite a matter of course that matter thus gravitationally entrapped in the interior of cosmic bodies is forever rendered subservient to the ever accumulating material overhead. It seems never to have suggested itself to the minds of the physicists that the burdened materials could ever find a way of release. Yet what deduction could be more simple than that, given a sufficient body of rock ripe for exploding, it would not need to await emancipation from the tardy hand of man, but, seeking the path of least resistance, would tear its own way, here or there, through to the surface? It is in this essentially explosible nature of matter, under varying pressures, that 
I find the chief cause of volcanic eruptions and earthquakes, and the solar eruptions.

Now, explosiveness is characterized by the abruptness of its happening. When the critical condition within the bowels of the earth is well advanced, but not yet altogether ripe for a spontaneous letting go, the impending catastrophe may be precipitated by some foreign agency, as, for example, by a great solar eruption-how will be explained later. The point to be impressed in this place is, that the exploding crisis is not indefinitely deferable, and that, sooner or later, the explosive tendency within the sphere catches up to and finally overwhelms the repressive forces. The effect of these belchings out of the earth's interior is to relieve the distension of her crust in the neighborhood of the orifices of escape and allow subsidence to the normal level; following which event the gases go on accumulating again, until the like action is repeated-a sort of earth-breathing, one might say. This alternate subsidence and elevation of the earth's crust is a long-standing scientific curiosity, and has heretofore been sought to be explained by the socalled principle of Isostasy; meaning, that the various areas of the earth's crust are equilibrated against each other. That this principle of isostasy is fundamentally sound, it seems to me, should go without saying; only it requires to be treated, not so much as a prime cause as a concomitant one of minor potency.

\section{The Anatomy of the Sun}

Returning again to the sun, my conception of him is that of an enormous bounding shell of molten matter enclosing and mightily compressing an inner spherical chamber of superheated gases, whose buoyancy, in turn, supports the shell. In short, the sun is, in principle, constructed on the order of the soap-bubble, only his walls are proportionally far thicker.-How thick?

It can be shown by a very simple calculation, based on the rule that the volumes of spheres are to each other as the cubes of their diameters, that were the third-of-a- 
million earths that compose the sun crowded, in their solid state, into his outer part, they would make a shell about 47,000 miles thick, equivalent to one-fourth his present volume. Of course this cannot be the case actually, seeing that these counterparts of our earth, as we are assuming them to be, are largely composed of substances too volatile to be able to resist the high solar temperatures. Moreover, we must allow for the weight of the gases occupying the central reservoir, and for the weight, also, of the solar atmosphere. It is not altogether guesswork if we allow for these claims about one-half the shell's thickness, reducing the latter to, say, 25,000 miles, in round numbers.

A few peculiarities of this shell demand attention. One of these has to do with the observed inclination of the sun's axis, which prima facie should, according to my theory of the stellar resultant and systemal equilibrium, parallel the axis of the earth. As a matter of fact, however, the solar axis is inclined, not $23-1 / 2^{\circ}$, but only $7^{\circ}$, to the plane of the ecliptic. What explanation can there possibly be for this apparently fatal discrepancy?

My answer is this: that were the sun rigid to the same degree as the earth, he would undoubtedly, like Mars, lean as does the earth, but not being rigid, he ceases ipso facto to be a trustworthy criterion of the main question. Prior to the ocular discovery of the sun's equatorial acceleration, and that from his middle latitudes to his equator this acceleration is gradual and continuous, and even reducible to a formula, what astronomer would have supposed such a thing physically possible? When, therefore, I say, as I now do, that the solar shell consists of many plies, and that each ply has its own velocity of rotation and its own PLANE of rotation, it may sound quite as incredible to the same ears. According to my interpretation, the innermost ply of the solar shell revolves about an axis nearly, or quite, parallel with the axis of the earth, while his outermost envelope, at the equator, revolves about one that nearly approximates a perpendicular to the plane of the planetary orbits. 
A second peculiarity about the sun that is not true of the earth is, that the former exhibits no oblateness of figure whatsoever, but presents at all times a sensibly circular disc. Were the sun as rigid as the earth, he would be flattened at his poles, but, because of his inflation, his rotundity is being perpetually retrimmed. The ball of our planet lacks elasticity and, as in the case of a ball of putty, its settling and flattening become more and more confirmed as time goes on. Were a close physical examination of the sun humanly possible, it would be found that the northern end, or base, of his shell is appreciably thicker and heavier than its southern, but this in no way affects his external symmetry, inasmuch as his interior buoyancy maintains his shell exactly centered upon his center of mass.

\section{The Solar Process}

Without pausing at this juncture to point out the source of the sun's heat, save to assert that it is a function of his mass, and judging him simply by analogy with the earth, whose temperature increases with the depth, we are justified in assuming that his temperature, too, rises steadily from his photosphere to his center. This naturally prompts the query as to what effect this condition has upon the character of the shell's chemical composition at the various depths. A preliminary illustration may assist in clarifying the answer:

Suppose you were handed a mixed pailful of finely pulverized, insoluble materials known to contain equal parts of ten different minerals, all evenly graded in an ascending scale according to specific gravity; how would you go about the task of separating them? Without bothering to cast about in our minds for an artificial way, let us put the burden on Nature and see how she would do it. We will search for a very deep and narrow well and, finding it, we will dump on its surface, as abruptly but as gently as possible, the entire contents of the pail, then with the eye of imagination, to which all things are transparent, watch the result. We will suppose, further, that 
the water in the well is just 10 fathoms deep. Clearly, there would ensue an interesting race between the ten substances for the bottom, in which contest the test of fitness would, of course, be that of weight, or more technically speaking, specific gravity; and when the affair has been concluded we shall find, piled up on the bottom, a column of ten thin layers ranged in the order of their heaviness. But what of the status of the race at its various stages? In the first fathom, beginning at the top, we shall see all the ten minerals inextricably mingled, but in this stretch the lightest of all will become so distanced that in the second fathom we shall be able to distinguish but nine sorts, in the third but eight, and so on until the heaviest particles begin to touch bottom.

Now, something of this sort occurs in the shell of the sun, except that here the tests of fitness are two instead of only one, and these two are, first, specific gravity (perhaps it were more accurate in this case to say atomic weight), and, second, refractoriness, or resistance to volatilization. Imagine now, if you please, the earth ground into fine powder and sprinkled over a limited area of the solar surface, and for the sake of simplicity suppose, further, this powder to be classifiable into ten distinct grades, as in our previous illustration, and, lastly, suppose the solar shell to be likewise differentiated into ten successive strata with temperatures progressing downwardly. Is it not obvious, now, that there would here ensue a process of distillation, a sort of refining or smelting process, in which the diverse materials would, in the order of their volatility, be successively driven off as they sank (by reason of their avoirdupois) into lower and lower levels of higher and still higher temperatures? It follows, then, as a matter of deduction, that the lining of the central chamber of the sun, or shall we say its facewall, is almost, if not quite, homogeneous and composed of a substance all but perfectly refractory, and that thence on outward the layers gain steadily in heterogeneity until we reach the surface.

In this process we see that the sun is something more than a dispenser of light and heat; he is also a smelter of 
minerals, providing man with the ores, which may not inaptly be termed cosmic pig metal. How these materials reach the earth we shall presently discover; for that they do, and that they have reached her in comparatively recent times is sufficiently attested, not only by the fact that these heavy ores are to be found here in "veins" and "pockets", but that they are to be found on the surface. Had the earth been originally her present size, and molten, the heavier materials must inevitably all have sunk toward her center and only froth and scum remained to blanket her.

Though I have spoken of the sun's strata, both in discussing his differential rotations and the chemical constitution of his shell, and shall yet continue so to speak, yet I wish the reader to understand that there are no sharp lines of demarcation between them, but that the changes up and down, though certain and pronounced, melt imperceptibly into each other. The reason lies, of course, in the great variety of the natural chemical elements and compounds, and in their abundance in the solar economy. Assuming that the graded temperatures of the shell, level by level, are invariable, and that the pressures are equally so, it follows that every element and compound regularly explodes every time it reaches its particular critical depth. Such explosions necessarily cause eruptions through the photosphere, giving rise to jets, geysers, fountains, or prominences, whichever the reader may think best to call them. In proportion to their deep-seatedness and severity, these explosive gases not only change places themselves, but they carry before them in their outward rush great quantities of debris from the intervening layers to greater or less heights beyond the photosphere, whence, being normally condensed and turned back by the cosmic cold, they rain down upon the photosphere in a ceaseless hail of slag and cinder-only to begin the same process over again.

Briefly, then, the solar process consists in the continuous explosive conversion of the sun's interior substances into gases by virtue of the pressure of the super- 
incumbent layers falling toward his center, the space allowing for such fall being vacated by the said gases, which rise to the surface upon the wings of their own explosive force, there render up their heat, condense, and regain their original energy of position as part of the great solar press - an unending cycle of mechanical combustion and gravitational resynthesis.

This cyclical process of converting gravity into heat and heat into gravity again, finds a remarkable parallel in the terrestrial transformation of water into vapor, rain and ocean, of which were one link unknown it would be infinitely more difficult to divine. In the solar heatgenerating cycle, the descending jets, or geysers, answer to our rain, perpetually flooding the whole of the sun's surface, and falling, with the regular continuity of the Amazon, toward his interior, there to suffer "evaporation" by his self-pressure instead of by his rays and thence to rise again in fountains of life-giving warmth.

It is plain to be seen that the shorter the distance that a substance must sink before reaching its critical level, the shorter the cycle of its action and consequently the more frequent its periodical eruptions. On the other hand, for that very reason these superficial eruptions are proportionately mild. It is from the pinnacles of these jets-ever freshly new, incandescent, and unencrusted-that we derive our extraordinary supply of light and heat. In short, they pump us heat and light, as it were; which accounts for two things; first, for the puzzling intensity of the solar radiations, and, secondly, for the keeping measurably cool, by their "exhaust", the magma from which they issue. They are doublecarriers, as it were, conveying their cargoes of heat out into the cold sky, exchanging there their cargoes of heat for cargoes of "cold", and bearing these latter back for the sun to expend his excess energy upon.

The so-called "rice grains" of the sun are nothing more or less than the geyser pinnacles above mentioned. They are the ephemeral ebullitions in the magma, appearing and disappearing in endless repetition and profusion. 


\section{Of these Professor H. H. Turner in his A Voyage in Space (p. 214) says:}

By a tragic accident he [M. Hansky, a Russian astronomer] was drowned, and no one else has paid the same attention to photographing these rice grains on the sun; but he obtained a sufficient series of pictures to show at what a great rate they are moving about. Even in a few seconds the pattern becomes quite differently arranged, as you can see by comparing one of M. Hansky's pictures with another. They must be moving at great speed, some of them perhaps at 100 miles a second $* * *$ The whole surface of the sun is in a state of constant turmoil.

And on p. 244 he adds :

When I tried these different ideas, I concluded that the particles (in the corona) were behaving as a fountain behaves, being both shot up and falling down again $* * *$ My conclusion is that there are a large number of solid particles in the corona, because there is a great deal of polarized light; and other astronomers have found the same thing.

So much for the superficial eruptions, but what of those more deeply seated? The cycles of these are naturally longer; all the more severe too, for that very reason. Late researches have led to the knowldge that the "solar constant" is not so constant after all, but varies rhythmically by periods of five days, of ten days, and so on, proving the occurrence of fluctuations in the sun himself. All of these variations are easily accounted for by postulating a series of eruptive levels, each with its own characteristic interval of manifestation.

\section{The Sun-Spots}

One of the periods of which we have been speaking is, of course, that of the sun-spots. In this phenomenon we have an example of sufficient distinctness and legibility to enable us, by a close study of available data, to determine the solar level from which it emanates. This is not exactly an easy task, for the reason that, as before stated, the blast carries before it in its ascent vast quantities of miscellaneous debris from all of the superior layers which requires to be differentiated out. The test, 
of course, consists in discovering which is the most refractory element or group of elements most conspicuously represented in the pertinent spectra; and a good deal also depends upon the age of the spot at the time the spectra are obtained.

Some may infer that I mean that these deep-seated blasts occur sporadically, and without any preliminary preparation of the surface. I do not mean this especially, though I admit the possibility of such happenings. The time being ripe for the coming event, I presuppose, rather, a series of eruptions, from the top layer to the next, and so on, opening a path of least resistance down to the lowest layer affected in each individual case. With each succeeding deeper detonation the original superficial vent is widened more and more until there yawns at last a well thousands of miles in depth, ripped through from below. This remains open until the internal pressure is sufficiently relieved, and then ensues a falling and filling in until the gaping wound is completely healed. That the girth of the sun should diminish slightly as a result of these belchings is self-evident, but the difference is too small to have been measured, though Professor Poor, it seems, has detected some such difference.

Owing to the differential rotations within the solar body, as previously described, and the friction caused thereby, the pent up gases are electrified before their escape, and, after escape, rising hundreds of thousands of miles above the sun's surface, they produce and perennially keep in repair the coronal veil that envelopes him. "There is a cycle of changes", says Doctor Abbot, "supposed to be identical with that of sun-spot frequency. As the corona can be observed only at total solar eclipses, the march of the cycle of changes is as yet only imperfectly known, but for the last half century it has been observed that there are long equatorial coronal streamers at the time of sun-spot minimum while at maximum of sun-spots the corona extends only to moderate distances but nearly uniformly in all directions from the 
sun". A comparison of the photographs he gives (The Sun, pp. 132, 134) will show that at sun-spot maximum the corona is very much more dense than at minimum, confirming the justice of my assertion that it is the emanations from the spots that renew the veil. Heretofore the Newtonians have shrunk from admitting this very natural conclusion, realizing as they did the impossibility of reconciling a materially dense corona with the non-retardation of the comets that have been seen to traverse it.

It has been observed, time and again, that solar eruptions on a grand scale are usually accompanied by magnetic disturbances on the earth, by tidal waves, by earthquakes, or by all of them together, and the causal connection has until now been one of complete mystery. It has, indeed, been suggested that the sun at such crises is exceptionally charged with electricity and that this somhow finds its way across the void; but this idea is recognized to be far-fetched even by its proponents. The true explanation is this:

Some sun-spots have been observed as much as five times as wide as the earth, and there have furthermore been witnessed from time to time solar eruptions upward of 200,000 miles in height, with initial velocities exceeding 100 miles a second. Such convulsions as these necessarily portend a substantial displacement of the center of mass of the sun and ipso facto of the entire solar system, and cause perturbations to every individual planet, satellite and comet within the latter, including, of course, our earth. This is bound to be the case, whether we view the planetary system in the conventional light of Newtonian astronomy or in the new aspect of an equilibrating unit. To make use of a homely simile, the sun at such times is like a great spider in the act of shaking his gravitational web. Now, (to change the figure) although we, the passengers, on this great ship may not be able to sense these perturbations directly, yet the earth herself does, and so while the solar convulsion lasts, she gently wavers in her orbit and sways 
upon her axis in rhythmical sympathy with her stricken lord. The inevitable result is that the oceans are caused to shake profoundly in their huge basins, producing not only tidal waves, but also, by the friction on their beds, augmenting the supply of electricity normally furnished by the diurnal tides. The effect of these perturbations on the solid parts of the earth is sometimes, though perhaps not usually, accompanied by earthquakes. The reason for this is not that the perturbations are the vera causa of, but that they precipitate, earthquakes nearly ripe for spontaneous manifestation, much as the slamming of a door may cause the glowing coals of a grate fire to collapse when near the point of doing so of themselves.

Knowing as we do by ocular evidence of the habitual periodical occurrence on the sun of these eruptions, big and little, the question suggests itself whether the sun may not be subject, at longer intervals than we possess records of, to vastly greater convulsions. A phenomenon that seems to lend color to this inference is that of "new stars". "These", says Doctor W. W. Campbell, "appear with great suddenness at points where previously no star of catalogue brightness (that is, as bright as the ninth magnitude) was known to exist, and occasionally, according to photographic observations, where no star as bright as the twelfth magnitude was recorded. They reach maximum brilliancy in a few days or a few weeks, pulsate through a considerable range of brightness for a few additional weeks, and thereafter decline more or less continously until they become comparatively faint stars. In some cases they assume approximate constancy as faint stars, and in others they seem to go beyond the reach of telescopic power, and later become visible again as faint objects". What is thus clearly true of some stars may reasonably be true of others, and of our sun; not simply once, but repeatedly.

Just such cataclysms as these have, as a matter of fact, occurred again and again to our luminary in the long geological past; not all of them, of course, equally severe, yet comparably so. Violent though they are, however, 
they are far from being total, nor should it be inferred that they occur at regular intervals any more than do the sun-spots. It is to these solar backslidings that I attribute the alternation of genial-and ice ages, for which scientists have so long been groping for explanation in vain; and to them do I also attribute, in chief measure, the enormous alterations of continental levels ushered in with the glacial epochs. Obviously, the sudden liberation of a great store of high-temperature gases would greatly reduce the sun's radiation for a long time to follow, and centuries would probably pass before he recuperated to normal, and still other centuries ere he could succeed in dissolving away the glaciers accumulated during his prolonged lapse. As for the changes of level, these may easily have been due to the convulsive shakings of the solar web. These would, as a matter of course, precipitate earthquakes on a grand scale, fracturing the crust in a multitude of places; and through the fissures thus created the imprisoned gases would make haste to escape, thereby not only giving rise to numerous volcanoes and much fouling of the atmosphere, but incidentally letting down the previously distended crust. Nor should we here neglect to take account of the lateral stresses due to the gravitation of the land masses toward the north pole, for it is just at such a time of equilibristic readjustment as this that this lateral pressure should make itself most felt and improve the occasion by raising up the great mountain chains.

The problem as to just how often these central explosions of the sun occur may find its answer in the response geologists shall give to the question as to the frequency of ice ages. If, in the absence of definite knowledge, we assume the earth to be a billion years old and the average interval between successive ice ages 100,000 years, there should have been, to date, 10,000 such ages and 10,000 such cataclysmal explosions. It is very doubtful, however, whether geology, on account of obliterations of its records, can supply a surer answer to this query than solar physics may more directly and speedily yield. 
With the escape of the buoyant gases from within him, the sun would naturally collapse upon himself, and there would follow a long period of rejuvenation during which his radiation, color and brilliancy would all be those of a star of lesser mass; and during this same period, which must be regarded as an abnormal and fitful stage, he would, furthermore, exhibit fluctuations in all the three attributes mentioned, making of him, for the time being, a typical variable star.

Although solar and stellar explosions are automatically produced by the causes just outlined, it does not follow that these are the sole agents. There must always lie in the back-ground the possibility of collision, not so much with another star, which is a very remote possibility indeed, but with a great comet. The comet of 1840-1841 may have been such a one, and had it been fired by its parent star a shade more accurately, it might have struck the sun amidship, punctured his inflated integument, and precipitated upon us a dire calamity.

\section{THE GENESIS OF THE SOLAR SYSTEM}

In such catastrophic explosions of the sun, then, be their causes what they may, we have the secret of the genesis of the nuclei of the planets and the method by which these nuclei have been gradually built up into the great bodies we now see. The planets were not created full-grown and full-panoplied, but they have added layer on layer, development to development. Indeed, every biological and geological fact proclaims the truth of this dictum, as every well-informed scientist will freely concede. The objections to the hypothesis of self-explosion on the part of the sun in the past and its heretofore total exclusion from consideration, have not been on account of any intrinsic defect, but because it left out of the reckoning the origination of the rotatory motions of the systems and, besides, contravened the dogma of the conservation of energy. The first of these objections is met by the newly-discovered principle of the Prime Resultant; the second will be considered in a following chapter. 
The pent-up gases, escaping as they do in these catastrophic cases from under a hydrostatic pressure of millions of atmospheres, in tearing their way through the superincumbent 25,000-mile shell naturally carry with them billions upon billions of tons of solar magma in various stages of metallurgical reduction, according to the solar level from which they respectively emanate. No doubt the major part of this upheaved material falls back upon the sun, but a vast amount of it is carried on outward-past Mercury, past Venus, past the earth, on even to Neptune, the outermost planet-spreading itself into a disc-shaped nebula as it goes; disc-shaped because the sun-spots are known to seek the solar equator. Indeed, some of these ejecta do not even remain within the system, but forge onward into space, distancing the solar gravity, as it were, and finally cross the border line of neighboring systems, there to become the cometary and meteoric vassals of other suns and stars. Inasmuch as the disruptive force is single, and the ether of space opposes no resistance, we should naturally expect that the outer planets would be fed proportionately more of the lighter materials of the solar carcass, and vice versa as to the planets nearer him. At any rate, this accords well with the physical facts, for the superior planets are by far the least dense.

The violence of the ordeal ended, the nuclei originally, and now the matured planets, under the gyratory rule of the Prime Resultant, continue on in the even tenor of their way and proceed to sweep up by their gravitational suctions the nebular material littering their respective orbital zones. It is quite true that the nebula, under the principle of systemal equilibrium, tends from the first to accomodate itself to the Keplerian law of areas, in which case, if that goal were immediately realized, the ingathering process would be defeated; but it is also true that this adaptation requires a long period, during which the differentiation of orbital speed between the coursing planets and the relatively stagnant medium immensely facilitates the gleaning process. In spite of 
this advantage, however, wisps of the most tenuous matter will always remain uncaptured and continue to litter the zone of the ecliptic indefinitely. These wisps, being illuminated by the sun outside the boundaries of the earth's shadow, are responsible for the phenomena known as the Zodiacal Light and Gegenschein. Incidentally, be it added, this residuary nebular matter is forever seeking to preserve its equilibrium, in pursuit of which it hovers near the plane of the ecliptic, as part of the main vortex; and where it pervades the earth-moon subordinate vortex, it affects the plane of the lunar orbit.

When I say that the planets devour this nebular matter, I do not mean to exclude from the cannibalistic feast. the asteroids, satellites, or even the meteors, that help to. make up our system. All of these share impartially, according to their respective gravitative voracities. Furthermore, they stand the same risk of being struck by the flying missiles. This suggests the question as to what should be the direct effect upon terrestrial life were such a catastrophe as I have described to take place today.

Granting that the earth is the built-up product of 10,000 such contributions from the solar mass, and that the same thing is true of all the rest of the planets and satellites, then, since all these together equal 1-750 of the sun, it follows that the latter loses on each such occasion only 1-7,500,000 of his corpus, or, say, 1-20 of an earth. Since the earth's dise, as previously computed, intercepts. only $1-2,000,000,000$ of the sun's light, of course it would likewise intercept only the $1-2,000,000,000$ of $1-20$ of six sextillions of tons of weight, or $150,000,000,000$ of tonsequal to 750 tons to the square mile, or, say, 1.25 tons to the acre, taking in the entire area of the earth's surface. Of course this solar hail would not be evenly distributed, unequal fragments being promiscuously scattered here and there, while the night side of the planet and other sheltered places might escape scot free. The real increment to the planet's growth would not be acquired by this direct bombardment, but by the subsequent slow gleanings from the nebula. 


\section{$\mathrm{XI}$}

\section{THE STARS AND NEBUL㞋}

66 T E made the stars also" is the curt phrase with which the Mosaic cosmogony dismisses these wonderful diadems of the sky, and, milleniums later, the great Newton himself knew nothing to add about them in the way of definite knowledge. During the past century, however, beginning with Bessel's and Henderson's successful determinations of the parallaxes of Alpha Centauri and 61 Cygni in the 1830's, the discovery of the principle of spectrum analysis by Kirchhoff in 1859, and Sir William Huggins' application of the Doppler principle to the study of spectra for determining the radial velocities of stars, in 1868, immense progress has been made-but, only, be it frankly said, in the way of gathering material, rather than in the work of actual construction. Let me particularize:

\section{Stellar Motions}

First, as to stellar motions. I trust I am not misjudging the aims of astronomers in supposing that their detailed study of individual stars and of their several proper and radial motions is not esteemed by them an end in itself, but a stepping stone, merely, to an ultimate understanding of the organic constitution of the universe as a unit and a system. Indeed, if the latter be not the true purpose, and if the mere multiplication of unclassifiable and unintelligible data of such things as, observations on variable stars, star spectra, radial motions of 
stars, proper motions of stars, distances of stars, magnitudes of stars, and the like, is the sole end in view, then in the name of common sense and wholesome economy let the farce be stopped. There is but one way to justify all this labor and expense of accumulation and to lift work of the same sort in the future above the plane of drudgery and mental dissipation, and that is, by finding a noble and structural use for it all.

Suppose that mankind, notwithstanding all the investigations with telescope and spectroscope hitherto made, should have continued in the belief that the earth is the center of the universe and that the firmament revolves around it daily; what genuine value would all our detailed knowledge possess? The knowledge both of the earth's rotation on her axis and around the sun is absolutely indispensable to a sane interpretation of the solar system. But it is no more essential to this purpose than is the knowledge of the form of the sun's path in space to a sane interpretation of the stellar motions and system. It constitutes all the difference between truth and error, between a yes and a no, whether the sun is speeding in a straight line or in a curve.

In asserting with such positiveness (v. q., p. 12, ante, the rectilinear nature of the sun's path Doctor Campbell has not only vented his own personal views, but has likewise stated the only logical conclusion that the Newtonian doctrines of uncaused motions and negation of equilibrium allow. About the only thing of great significance that this study of the proper and radial motions of stars-a study which represents a perfectly incredible expenditure of valuable time, skill, energy and moneyhas developed, is the supposed existence of star-streams, for which the investigators confess their inability to suggest any explanation, and whose existence runs counter to every Newtonian tradition. Under my system of interpretation these streams are not streams at all, but an optical illusion created by the movement of our solar system in its gyroscopic course from east to west and the fall of the ecliptic; much as the ancient notion that the 
heavens rotated daily around our earth was such an illusion. By parity of reasoning, the so-called vertices of preferential motion are likewise the effect of identically the same cause; and so, likewise, is the locus of the solar apex, as hitherto determined, apparent merely, and not real. The true course of the sun is that of recession (westward) from the celestial point of the vernal equinox along the equinoctial colure; the latter's 'length (186,000,000 miles) constituting an are of about $50^{\prime \prime} .2$ of the sun's orbit, having its concave side toward the position of our earth in winter, and continually sinking toward the Vertex, or gravitational pole of the ecliptic, at the rate of some six miles per second. In attempting to visualize this description and to truly appraise the illusory character of these "streams" the reader is cautioned to take into the reckoning the phenomenon of precession at its face value, and not to discount it, as astronomers now do, under the rulings of Newton's misconception of its cause. The sun, under their conception, should seem to tend toward R.A. $270^{\circ}$ and Dec. $+33^{\circ} .23$, but his actual path is as I have described.

Generally speaking, the movements of stars are far too slow, and the labor of ascertaining them in the first instance, and of afterward piecing them together, far too tedious and difficult, ever to make the general mapping out of the stellar field into minor individual systems well worth while or, perhaps, possible. Enough of this sort of work has already been done, however, and more is now in progress, to reveal the tendency of vast star-clusters to arrange themselves into spiral conformations, indicating the operation of some common cause. What, I pray, can this cause be other than gravitation-not centripetal attraction by itself, which logically should destroy the isolated system (all the faster because of its isolation), but that attraction in combination with the stellar resultant reinforced by the equilibristic principle: The clue to all such observed arrangements is to be found, if at all, in first determining as nearly as can be from observation the directions of the shortest axis and of the 
longest axis of the given cluster, and then angling for the line of the prime resultant that best fits the situation. In this connection it will be illuminating to compare this passage from Professor Proctor's book, already referred to (Our Place Among Infinities, pp. 201-202):

Father Secchi of Rome speaks thus of the distribution of stars within a certain very bright portion of the Milky Way in the constellation Sagittarius, as revealed by the powers of the fine refracting telescope of the Roman Observatory:- "There are large stars and lucid clusters; then a layer of smaller stars certainly below the twelfth magnitude; then a nebulous stratum with occasional openings." But what startled him and all to whom he showed it, was the regular disposition of the stars in figures so geometrical that it is impossible to regard them as accidental. "They are for the most part like the arcs of a spiral; one can count as many as ten or twelve stars of the ninth and tenth magnitude-following each other on the same curve like the beads on a rosary; sometimes they seem to diverge from a common center, and, strangely enough, it usually happens that either at the center of the rays, or at the beginning of the branch of a curve, there is a larger star of a red colour. It is impossible to regard such an arrangement of the stars as accidental."

That scholastic astronomy, notwithstanding its wealth of detailed knowledge, remains primitively ignorant of the general principles governing the movements of stars, as well as of the structure and internal activities of the individual stars, and that some radical reform in astronomical theory is imperatively called for, will appear from the subjoined quotations. The first of these is taken from Doctor Campbell's book (p. 216):

It is not easy to explain why the velocities of stars should increase with their effective ages, for we are accustomed to think of all matter as equally old gravitationally. Why should not the materials composing a nebula or a Class B star have been acted upon by gravitational forces as long and as effectively as the materials in the Class M stars? Are stellar materials in the antestellar state subject to Newton's law of gravitation? Does gravitation become effective only after the processes of combination are well under way? Is it possible that the gaseous matter composing a nebula is acted upon as effectively by radiation pressure as by gravitational attraction? The observed fact of the dependence of stellar velocity upon the spectral class is so new that these comments and questions make no pretensions to the 
status of a solution; but I am unable to suggest any other directions in which we should seek for the explanation.

And the second is from the recent work of Professor A. S. Eddington, of the University of Cambridge (Stellar Movements, p. 255) :

The problems on which dynamics would be expected to throw some light are numerous. Why have the stars in the early stages very small velocities? Why do these velocities afterwards increase? In particualr, how do the stars acquire the velocities at right angles to the original plane of distribution, which cause the latest types to be distributed in a nearly spherical form? How are the two star-streams to be explained? What is the meaning of the third stream, Drift $O$ ? Can the partial conforming to Maxwell's law be accounted for? What prevents the collapse of the Milky Way?

Some of these problems seem to be at present quite insoluble. Indeed, it must be admitted that very little progress has been made in the application of dynamics to stellar problems. What has been accomplished is rather of the nature of preparatory work. It has been shown that stellar dynamics is a different study from gas-dynamics, and, indeed, from the theory of any type of system that has yet been investigated. A regular progression may be traced through rigid dynamics, hydro-dynamics, gasdynamics to stellar dynamics. In the first all the particles move in a connected manner; in the second there is continuity between the motions of contiguous particles; in the third the adjacent particles act on one another by collision, so that, although there is no mathematical continuity, a kind of physical continuity remains; in the last the adjacent particles are entirely independent. A new type of dynamical system has therefore to be considered, and it is probably necessary first to work out the results in simple cases and to become familiar with the general properties, before attempting to solve the complex problems which the actual stellar universe presents. This has been the mode of development in the other branches of dynamics.

\section{Star Structure and Characteristics}

Granting to all stars the same chemical constitution in kind and ratio, I hold that their temperature, density, velocity, color, and brightness are all functioned on the mass, and, further, that their spectra are ordinarily reliable criteria of their masses. By this I do not mean that there is any exactly commensurate relationship or 
correspondence between all of these characteristics, but merely that, the bigger the star the hotter its temperature, the thinner its density, the slower its velocity, the whiter (or more bluish) its light, the brighter it is, and the more refractory its normal spectrum. This rule is subject to one general qualification, namely, that all stars are liable to collapse (as a result either of spontaneous explosions due to clogged radiation, or of puncturing by flying missiles from other stars) and also to variations (e. g. sun-spots), which are in the nature of collapses, only minor in degree and quickly periodical in recurrence. In these collapsed or semi-collapsed states, the star, by reason of the sudden escape of great quantities of its superheated gases, drops for a spell into a lower order of stars, not so much because of the actual loss of substance, which is relatively insignificant, but because of its decided fall in temperature, whereby its phenomena are for the time being modified. We will now consider the various characteristics separately.

Temperature. According to current notions, based as they are on the doctrine of the conservation of energy, all the stars, the sun, and the planets are in the process of cooling down from an initial state of incandescence; hence, argue the scholastics, the duller a star the older. In the next chapter I shall attack this law of conservation and expose its hideous absurdity, establishing in its stead the proposition that heat is a staple product of Nature's, and that gravitation, as represented in quantity of mass, is a perpetual generator of it.

Density. Were the density of every cosmic body the very same, then mass and volume could be treated in mathematical computations as synonymous terms. It is, however, far from being the same; each planet having its own particular density, the sun having his, and every star presumably having its. There must be some physical cause or causes for this contrariety, What are they? These three occur to me: (1) Difference in chemical constitution, (2) Self-compression, (3) Expansion by heat. 
That there is considerable difference in the chemical composition of the celestial bodies-if not in the number of elements represented, at least in their relative proportions-seems altogether likely; but there is no way of ascertaining the extent of this difference in any particular case. Some of my readers may suppose this knowledge obtainable from the spectra of the stars, but these give no clue whatever as to either the quantities, absolute or relative, nor, since the revelation of an element in the spectrum depends on the temperature intensity, do these spectra even settle the problem as to the mere number of elements. Except, therefore, where we can assign some definite ground for hypothesizing otherwise, we have no option but tentatively to assume that all stars, if not exactly, are yet approximately enough alike chemically to validate comparisons between them on this basis.

Having thus ruled out the factor of differential chemical composition, not because of its immateriality but because of its indeterminateness, we are relegated to the two remaining factors of self-compression and temperature. These two I associate, making the latter depend on the first as a causal effect. Construing density to decrease with temperature increase, we may begin by imagining all stars cold to start with, and all of them virtually of the same density. This last would, of course, not be exactly true, inasmuch as the larger stars, because of their more powerful self-compression, would necessarily pack their contents into smaller compass; but this, you can see, is a negligible consideration inasmuch as the condition requiring to be elucidated is not why the big bodies are more compact but why they are vastly less compact than the small. As said before, then, density being taken as initially the same for all bodies, mass and volume become synonymous, and we have for the ratio of the integral attractions of cosmic bodies in general the formula,

$$
\frac{\mathrm{M}}{(\sqrt[8]{\overline{\mathrm{M}}})^{2}}: \frac{m}{(\sqrt[3]{m})^{2}}
$$

in which $\mathrm{M}$ is the mass of the large body, $m$ that of the 
smaller, and ${ }^{8} \sqrt{\mathrm{M}}$ and ${ }^{8} \sqrt{m}$ are their respective radii; the latter terms being squared and placed under the line in conformity with the law of inverse squares. Substituting, by way of illustration, the sun's mass for M and the earth's (unity) for $m$, and working the problem out, we shall find that the sun's integral attraction (were his density the same as the earth's, instead of only onefourth, as it is) would be 69 times as great, whereas, computed on the basis of his real density, this ratio is only 27.6 to one! What, I pray, becomes of the tremendous surplus of this potential attraction (by which name we will hereafter refer to it) over the observed? So far as my reading has extended, I have found no suggestion about it anywhere, much less an attempt to fit it into the web of scientific theory. Yet what could be more likely a priori than that this surplus energy is utilized in the wise economy of nature for two things, first, to gaseously inflate the sun for certain cosmic ends, which I have already outlined, and, secondly, to generate the light, heat, and, perhaps, magnetism that is radiated so lavishly to the planets? Without going into the complicated minutiæ, it can be shown by mathematics that the density of stars diminishes as the "potential attraction" increases, and in making this statement I am not unmindful of the apparent, but only apparent, contradictions of some of the planets; and by the same process of computation, it can further be proved that the shell, though it increases in absolute thickness, diminishes relatively to the length of the radius with the star's growth.

(In passing, be it said, the reason why the sun's density seems to be greater than that of the major planets is because the ball of the former being luminous, we see it directly, despite his tremendously deep and dense atmosphere. Were the ball to lose its brightness, we should see him as a dark disc whose diameter would appear much greater because it would then include the thickness of his atmosphere; and his density would suffer in the computation accordingly). 
Velocity. Owing to two misconceptions hitherto prevailing, namely; (1) that dull stars are old, and hence presumptively massive, and (2) that proper motions are "inherent", Newtonians have been unable to formulate any rule whatsoever regarding stellar velocities. These latter, in their philosophy, are as capricious and arbitrary as the "inherent" motions themselves. The new principles which I submit are these:

(1) It follows mathematically from Newton's law of inverse squares and the law of the energy of motion, that if two bodies of different mass be conceived as occupying space alone, they will approach each other with velocities inversely proportional to the square roots of their respective masses. Thus dull stars being according to my hypothesis small, they are therefore quick moving.

2. Save in the cases of binary stars, where the circulatory relation is patent, current astronomy regards all star movements as rectilinear and dynamically independent. On the contrary, I regard all the stars as organically interrelated in the greater-cosmos, much as the individual soldiers are constituent parts of a great army. Thus, the earth and moon form by themselves the simplest of all systems-a binary; the sun and his planets another, more complicated; the sun and one or more of his near neighbors a still higher system, whose extent and membership we have not yet been privileged to discover-and so on. You can see here that the minor members necessarily move more rapidly than the major, for the substantial reason that they have all the various motions in cumulation. It is therefore only to be expected that the smaller stars, in whatever relation they may find themselves, should, caeteris paribus, travel with greater celerity than the more cumbrous; as, in fact, they do. Star velocity, then, is a function of the mass, not directly, but inversely as the square roots.

Brightness. Finally, as to the intrinsic brightness of stars. If it were possible to determine which is intrinsically the brightest of all stars, it would be equal to 
finding at the same time the very hottest and the very biggest as well-biggest not only in girth but in mass. Furthermore, it would be the least dense, and (less certainly) the most sluggish in movement. The reason for adding this last parenthesis is that pivot-stars (by which I mean such as, by reason of their relatively great size, preëmpt the central positions) have little motion besides that imposed by the direct action of their stellar resultant, whose strength varies, of course, in the different regions of the heavens, and may possibly attain, in exceptional instances, a high maximum.

"But", you may ask, "how are we to distinguish between those stars that are intrinsically the brightest and those that are brilliant only because of nearness?" The answer is twofold: (1) by the color of the given star, and (2) by its spectrum; which characteristics in all cases corroborate each other. The more refractory the spectrum (that is to say, the more involatile the elements it reveals) the brighter the star in itself. In choosing the monuments, therefore, for celestial surveying, care should be taken to select a series of Class B stars (which, according to my interpretation, are the very largest), whose color and spectra both are nearest alike. Of course the visual magnitudes of these stars, owing to their diverse distances, will vary widely, even though their intrinsic brightness, as I claim, is quite the same. It can readily be seen, then, that in this scale of differing apparent magnitudes based on a choice of stars equally bright intrinsically and of the same order of size, we have a most satisfactory and reliable means to gauge the stellar distances; and, secondly, inasmuch as these stars are by parity of reasoning the slowest-moving, we have at the same time reasonably stationary celestial pins from which to estimate the wanderings of stars less stable. This principle need not be limited to Class B stars, but can be applied whenever desired and convenient to a series of stars drawn from any other isochromatic spectral class.

It follows deductively from the premises that the bigger stars, being by hypothesis hotter for that reason, 
volatilize a correspondingly larger percentage of their component substances; thereby increasing the size of the inner chamber of gases relatively to the thickness of the shell for these reasons, namely: (1) by direct augmentation of the quantum of the gas at the expense of the shell, (2) by enhanced expansion of that gas due to the increased temperature, (3) by pushing the shell out that much farther, thereby enlarging the area over which the shell-substance must spread itself, and (4) by virtue of the circumstance that the weight of the shell, under the law of the inverse square, diminishes with its distance from the center, rendering easier its further uplifting. But let us now consider the effect of all this on the texture and conduct of the shell itself:

In the first place, the gradual distillation-out of the more volatile substances necessarily simplifies its chemical constitution and correspondingly affects the quality of the spectrum.

Secondly, the increasing predominance in the geysers of the more refractory materials which, as we all know, in their incandescent state are dazzlingly brilliant, naturally augments the luminosity of the star as a whole.

Thirdly, the hotter the shell the more plastic or fluid is it; hence the freer the ebullitions, the less liability to clogging, and the more multitudinous the geysers, whose pinnacles it is that cast off the radiance. This feature not only helps to account for increased brilliancy of stars with growth, but explains why the very bright stars are never found among the long-period variables, save, of course, those of the eclipsing variety.

Finally, the fact that the shell of a star, notwithstanding its increase in absolute thickness with the star's growth, declines relatively to the other stellar dimensions, cannot fail eventually to weaken the star's structural stability and to bring about its automatic bursting and dissolution into a nebula. Nebulæ thus evolved are obviously of enormous extent, not only because of the maximal size of their parent stars, but also because of the maximal pressure under which the central 
gases were confined and from under which they all the more violently escape. There is, then, an arbitrary limit imposed by Nature beyond which no star can grow, howsoever favorably it may be situated for further growth, and howsoever stout may be its armor against cometary missiles from without. Here, in this simple explanation, we have harmoniously coördinated the following important considerations: (1) How the greater nebulæ are formed; (2) Why stars, though differing greatly in mass, yet observe a certain order of size, like trees and animals; (3) Why there is not and can never be a great "central sun"; (4) The dispersive force by which the destructive centralizing tendency of gravitation is counteracted and the balance of Nature automatically maintained. Just how large a star may become is another matter. Even this problem, however, is susceptible of mathematical determination within reasonable approximation, though we cannot stop to deal with this complex problem now. In my opinion Doctor Campbell greatly overestimates the possibilities, when he says (Stellar Motions, p. 158) :-

The parallax of Canopus can scarcely exceed .oI or .02 of a second as a maximum. We can scarcely doubt that Canopus is radiating certainly 1000 and perhaps 100,000 times as much light as the Sun. If the effective radiating power of its surface equals that of the Sun, the surface must be fully Iooo times as great as the Sun's. Its corresponding volume would be 31,000 solar volumes. Its mass must greatly exceed the Sun's mass, probably between 1000 and 30,000 fold.

When stars of very large size, and of precarious structure because of their relatively thin shell, are subject to the stress of an especially powerful stellar resultant of short stem (a condition which occurs when many stars are in close proximity), they are forced to rotate on their axes with extreme rapidity, enough so at times as to flatten them out sufficiently thin to cause their poles to coalesce, and so to bring into being two stars of smaller size. This phenomenon is called starfission and, though rare, is well-authenticated by the observers. In the majority of cases, it appears, this 
unique feat of surgery, when Nature attempts it, turns out a success, the sundered ends are successfully sealed and cauterized, and the severance of the celestial Siamese twins becomes an accomplished fact. However, there is at least one case where a failure in this sort of cosmic chirurgery seems to have been scored and resulted in a double-nebula instead. Harking back to Darwin's tidal evolution theory, the reader may argue, as indeed Darwin and his adherents do, that since the fission of stars can and does take place, the earth might have flung off the moon. The cases, however, as the sincere reader will see on second thought, are not parallel at all. In this connection compare this interesting passage from Doctor Campbell's book, (p. 291):

The two stars [Beta Lyræ] are enormous in size, but of very low density. They are so close together as to be almost in contact. The two bodies are in form approximately prolate ellipsoids, with their longer dimensions in the line joining the two bodies. The immediate cause of the variable brightness is due, in large part, to the eclipsing of one body by the other, but there are probably other factors entering to a minor degree, such as tidal ebb and flow, which must exist, as the orbit seems to be slightly eccentric.

\section{VARIAble Stars}

In a sense all stars may be said to be Variable, for all alike have explosive graduated shells. In the very large stars, however, the shells are much more homogeneous and much more fluid, so that the ebullient action is practically uniform. I am speaking now, not of eclipsing variables, whose changefulness is manifestly due to the intervention of companion bodies, but of those whose flickering is due to a change that goes on in the star itself. In the previous chapter I have already given the reader my version of the true nature of sun-spots and the reasons for their periodicity, and he has only to apply the same principles to the analysis of particular cases and phases of star variation. In this engrossing study, however, two exceedingly important points must be ever borne in mind, namely; (1) that a very high percentage 
of variables are abnormals, and (2) that abnormal stars are invariably subnormals. By this I mean, that after the collapse of a star, whether by spontaneous eruption or by puncture, it for a time drops to the temperature of stars of lesser mass, and, secondly, that in this collapsed condition it is bound to exhibit changes in its lightespecially fitful changes. For illustration, compare the similar behavior of a coal or wood fire. Let it, however, not be concluded that only recently collapsed stars are subject to these variations, for our own sun is now in a normal stage for a star of his size, yet his sun-spot cycle is certainly a true variation. Apropos of this subject, let me quote another passage from Doctor Campbell (Stellar Motions, p. 283) :

A study of the periods of variable stars brings out most curious relations. The periods clearly have preferences for certain lengths. There are a large number whose variations from maximum to minimum and back to maximum are completed in approximately one day, and many whose periods are half a day or less. As the period lengthens from one day, the number of variables decreases rapidly until we reach the few of eleven-day period. There are relatively very few variables with periods between II days and I Io days. Variable-star nature seems to abhor this interval. Beginning with I Io days, the number of variables increases rapidly, with increase of period, up to a maximum at 345 days; and the number of variables then decreases rapidly until we reach periods in length approximately 450 days. There are few with periods longer than 450 days, and our information concerning them or their periods is extremely meagre. It is of interest to note that Omicron Ceti, with average period 33I days, is but one of a great number which make up the maximum near 345 days.

Long-period variable stars differ from short-period variables in important particulars. The former vary in brightness through a wide range, usually from three to eight visual magnitudes. Short periods, on the contrary, have small ranges of brightness, varying through 0.2 of a magnitude or less up to a maximum of I $1 / 2$ magnitudes, with few exceptions. The long-period variables are all reddish in color, apparently indicating that the atmospheres of these stars are dense, absorbing the violet rays and transmitting the waves of longer length, or that we are dealing with low-temperature radiations. Short-period variables, on the contrary, are all yellow or white in color. Chandler has found that there exists a relation between the length of period and red- 
ness. To quote him: "The redness of variable stars is, in general, a function of the lengths of their periods of light variation. The redder the tint, the longer the period." Whether the conditions which produce redness are also the cause of long periods is an unsettled question. These long-period red variables do not conform to definite time schedules. Their maxima may precede or may follow prediction by a fortnight or a full month, but the average length of twenty-five consecutive periods will differ almost not at all from the average of the twenty-five preceding or following periods.

Of the reasonably bright short-period variables there are about Ioo which pass from maximum to minimum and back to maximum within less than thirty days; nearly all of these, within ten days, and some of them within a few hours. In all these cases maxima and minima arrive on time, and the periods of most of them are known within a second. One cycle of change is almost exactly a duplicate of the preceding and following cycles.

The key to all changes and states of brightness in stars obviously lies in two things, namely; (1) in a close analysis and comparison of the pertinent spectra, and (2) in a systematic determination and tabulation of the luminosities of the various minerals (metals, more especially) at different temperatures. I note that some work along this latter line has already been attempted, but nearly all of it remains to be done.

Entering into the matter is this feature also: Inasmuch as, according to my version, the light of a star comes to us, not from its level surface but from its ascending geysers, it follows that the swifter the velocity with which these rise toward us the higher radial velocity should the star's spectrum indicate. Furthermore, where the star is variable it should appear at its maximum brightness when its deeper-seated, more refractory, substances are ebulliated; which is to say, when the geysers are thrown up highest and fastest. This reasonable deduction is curiously confirmed by the action of the classes of variables known as Cepheids and Geminids; speaking of which Doctor Campbell says (p. 306):

Interpreted, we have the astonishing result that every star investigated has its maximum brilliancy at or very near the time of greatest velocity of approach toward the solar system, and the 
minimum brilliancy at or very near the time when the bright star in the system has its maximum velocity of recession from the solar system. These are keys which give promise of unlocking many secrets of the Cepheids and Geminids. What can be more remarkable than that variable stars of this class should be at their brightest when they are moving rapidly toward the observer and at their faintest when they are moving rapidly away from him?

\section{The Nebulae}

Nebulæ are at once the ghosts of departed stars and the corpora of other stars yet to be born. They are not primordial, but transitional forms; the first star preceded the first nebula. They differ fundamentally from the original cosmic dust in having passed through the stellar crucible and consequently undergone considerable chemical rearrangement and combination. Furthermore, although much of it may have been lost in the cataclysms that gave them birth, they retain in their nebulous condition some remnant of the motion possessed by them as member stars of the systems that have vanished.

Owing to their explosive origin, nebulæ are naturally capricious and nondescript in form, and there is little to be learned from their incipient shapes. Their sizes, however, vary so widely as to demand categorical explanation. I have already recounted at length the production of the smaller nebulæ by the spontaneous explosion or puncturing of stars, and of large nebulæ by the bursting of maximum-sized stars from sheer over-growth. All of these, however, might perhaps result in rather too simple and regular forms to suit every nebular outline, and there might remain also a doubt as to whether the destruction of even the biggest star would commensurate with the size of the biggest nebula. To account for the extraordinary cases, then, such as Eta Argus or Orion, for example, I suggest that the explosion of some monster star in the near vicinity (as stellar distances go) of a cluster of other large stars might cause the puncture of several more, each succeeding explosion, moreover, creating a new center of destruction. In this way any 
size or irregularity of nebula that the heavens may present to the telescopic eye can be accounted for.

No sooner is the nebula spread out-nay, while it is yet in the very act of spreading-it begins to react to the subtle but all-powerful influence of its stellar resultant and to struggle within and upon itself for gravitational equilibrium. This, of course, is a tediously slow process, for the reason that the projectile momenta of the ejected material require to be mastered and disciplined. However, fortunately for science, we do not need to wait to see one particular case through before arriving at conclusions, since there are many thousands of these curious objects in the sky in different stages of deployment and evolution, so that by putting two and two together we can write the individual histories of all. Hitherto the teaching has been, that the moment of momentum of any segregated system cannot change, and that a nebula is to all intents and purposes a system and amenable to the rule. But that here, again, fact and theory do not gibe, note these words of Kapteyn:

The phenomenon of the increase of velocity with the evolutional stage of the stars must give rise to speculation as to its cause. The observational results contained in our table naturally lead us to conclude that the matter from which the stars originate must have little or no velocity. How is this possible under the influence of the combined attraction of the rest of the system? Is it not as if gravitation had no effect on the cosmical matter in its primordial state? If this be so, as soon as matter changes from this state to another in which gravity begins to act, or to act freely, motion will arise, and it is evident that, as a rule, the motion must be accelerated, at least during immense periods, so that the longer the period lapsed since the birth of the stars the greater must be their average velocity.

It would take us too far afield to enter into a detailed discussion of the numerous capricious forms of nebulæ, caprices that the very nature of their origin as hereinbefore set forth sufficiently accounts for; and we shall therefore confine this inquiry to the far more important consideration of the origin and significance of spiral nebulæ, which obviously involve structural dynamics of basic character. These peculiar nebulæ so far outnumber 
all others put together that the inference is unescapable that they are the advanced stages of a cosmic process by which all nebulæ are being molded. They are simply systems of planetary bodies enshrouded in star-dust expelled by the explosion of their central sun or suns, which they are gradually redding up by their gravitational suction while regularly rounding their orbits. To visualize such a spiral nebula you need only imagine our sun to explode profoundly, and then picture in your mind's eye what convolutions should take place in the nebula by the action of the planets. The exaggerated sizes predicated of some of the nebulæ are no hindrance to Nature's disciplinary powers. Be a nebula as large as it may, it still has its master stellar resultant, it still remains subservient to the inexorable law of equilibrium, the law of the balance-arm, Kepler's laws, the law of the logarithmic spiral, the law of gravitation-all one and the same in essence. By way of exemplifying these remarks let me cull still another extract from Professor Eddington's book (p. 241) :

It is generally believed that the spirals predominate enormously over the other classes of nebulæ; and, as the whole number of nebulæ bright enough to be photographed has been estimated by E. A. Fath at 160,000 , they must form a very numerous class of objects. They are seen by us at all inclinations, some, like the Whirlpool Nebula, in full front view, whilst others are edge-on to us and appear as little more than a narrow line. An example of the latter kind is also illustrated in Plate IV. In all cases, where it is possible to discriminate the details, the spiral is seen to be double-branched, the two arms leaving the nucleus at opposite points and coiling around in the same sense. From the researches of E. v. d. Pahlen, it appears that the standard form is a logarithmic spiral. The arms, however, often present irregularities, and numerous knots and variations of brightness occur. Unlike the planetary and extended nebulæ the spectrum shows a strong continuous background; bright lines and bands are believed to occur, at least in the Great Andromeda Nebula; hut they are of the character of those found in some of the early type stars, and are distinct from the emission lines of the gaseous nebulæ.

The distribution of spiral nebulæ presents one quite unique feature; they actually shun the galactic regions and preponderate in the neighborhood of the galactic poles. The north galactic pole 
seems to be a more favoured region than the south. This avoidance of the Milky Way is not absolute; but it represents a very strong tendency.

In the days before the spectroscope had enabled us to discriminate between different kinds of nebulæ, when all classes were looked upon as unresolved star-clusters, the opinion was widely held that these nebulæ were "island universes", separated from our own stellar system by a vast empty space. It is now known that the irregular gaseous nebulæ, such as that of Orion, are intimately related with the stars, and belong to our own system; but the hypothesis has recently been revived so far as regards the spiral nebulæ. Although the same term "nebula" is used to denote the three classes-irregular, planetary and spiralwe must not be misled into supposing that there is any close relation between these objects. All the evidence points to a wide distinction between them. We have no reason to believe that the arguments which convince us that the irregular and planetary nebulæ are within the stellar system apply to the spirals.

It must be admitted that direct evidence is entirely lacking as to whether these bodies are within or without the stellar system. Their distribution, so different from that of all other objects, may be considered to show that they have no unity with the rest; but there are other bodies, the stars of Type $M$ for instance, which remain indifferent to galactic influence. Indeed, the mere fact that spiral nebulæ shun the galaxy may indicate that they are influenced by it. The alternative view is that, lying altogether outside our system, those that happen to be in low galactic latitudes are blotted out by great tracts of absorbing matter similar to those which form the dark spaces of the Milky Way.

If the spiral nebulæ are within the stellar system, we have no notion what their nature may be. That hypothesis leads to a full stop. It is true that according to one theory the solar system was evolved from a spiral nebula, but the term is here used only by a remote analogy with such objects as those depicted in the Plate. The spirals to which we are referring are, at any rate, too vast to give birth to a solar system, nor could they arise from the disruptive approach of two stars; we must at least credit them as capable of generating a star cluster. $* * *$

The two arms of the spiral have an interesting meaning for us in connection with stellar movements. The form of the arms-a logarithmic spiral-has not as yet given any clue to the dynamics of the spiral nebulæ. But though we do not understand the cause, we see that there is a widespread law compelling matter to flow in these forms. 


\section{Nebula- and Star Spectra}

The manner of the formation of nebulæ, as just described, seems to me to supply the key to the peculiarities of thuir spectra. For the freshly liberated gases will be expelled to unequal distances, and the lighter elements the farther. The outer envelopes of the nebula should therefore consist largely of hydrogen and similar gases; and if these envelopes be sufficiently dense so as to be opaque to the light of the other incandescent elements ranged behind them, the predominance of such lines in celestial spectra should cease to surprise. Given sufficient allowance of time in which to act, gravitation would of itself dispose the elements in the same order, that is, the lighter on the outside.

The so-called "green" nebulæ are therefore younger than the "white", and, by the same token, naturally larger, from not having been condensed by long exposure to the cold of space. Their youthfulness is further attested by the fact that less than five per cent of nebulæ are green, a circumstance which opens interesting avenues to the determination of the relative ages of nebulæ in general. The Milky Way, being the densest portion of the sidereal system, is naturally richest in all sorts of nebulæ, but particularly in the percentage of those of this green, or ultra-gaseous, type. The difference between the various classes of nebulæ is therefore accidental rather than fundamental or generic. The manner of their formation (that is, by explosion) naturally arrests and masks such proper motions as their parent star may have had just prior to the catastrophe, so that the new-born nebula is obliged to "find itself", as it were, and to acquire its new motions by a protracted process of gravitational acceleration. As the "dust from the explosion" settles and clears, the nuclei, which were always there, but only hidden from our sight, loom gradually into view, and then we perceive a spiral nebula, evenly poised like a giant pinwheel on a center, recrudescing into a new cycle of existence. 
The double spectra of stars are never visible except where two bright stars are knowingly examined at one time, as in the case of binaries, or where there is evidence of some special eruption in progress on the body under examination. In the latter case the confusion of spectra is due, I opine, to the violent geyser-like ejection of gas from one section, while the other part of the surface remains normal. A suggestive illustration of this may be seen in certain comets, which now and again exhibit luminous spurs, or jets, directed toward the sun. Moreover, geyers that rise must concurrently fall back by the action of gravity, thereby producing double spectra, indicating both plus and minus radial motions.

\section{The Rings of Saturn}

In the large sense, all celestial bodies may fairly be included under the generic name of stars, for even the smallest of them may conceivably be the embryo of a Canopus or Arcturus gestating in the fertile womb of Time. Such enormous planets as Jupiter and Saturn are not very distant approximations to lucid stars, as may be inferred from the phenomena of the "great red spot" characterizing the former, and the "rings" that render Saturn so gloriously unique among telescopic objects. My explanation of these wonderful appendages is that Saturn has, in comparatively recent times-probably as late as the Neolithic age-been profoundly shattered by an explosion, from causes such as I have outlined, thereby giving birth to a genuine, if relatively minute, nebula, which, under the tutelage of the Prime Resultant subsequently took on permanent vortical motion. 


\section{XII}

\section{GRAVISTATIC HEAT}

T $\mathrm{N}$ earlier chapters we have seen how grossly Newtonians have been undervaluing the functions of the principle of universal gravitation in the domain of planetary and stellar motions. They have employed it negatively rather than positively; destructively rather than constructively; as a clog, a brake, a restraint, instead of as a never-failing well of motive power. They accept the so-called rectilinear motions as gifts out of the oceult, not in the single particular of momentum or velocity, but in those of adaptation and direction as well; and they employ gravity merely to lasso and tether the self-flying stars and planets. In the same way they postulate the rotations of the sun and planets as ultimate facts, or, at least, as conditions inherited from prior motions which themselves were self-existent; and then they invent an explanation of tides in which the role of gravity is to retard those rotations and eventually to bring them to a standstill.

Furthermore, we have seen how, following out this medieval style of speculation, and perceiving, but misinterpreting, the exquisite balancing of our system from day to day and year to year, they invented another abstraction, namely, the law of conservation of moment of momentum, which is conceived to control celestial motions, not through the ordinary channels of physical cause and effect, but by some sort of teleological ordination or oversight vested in abstract energy as matter's 
master and mentor. But this preposterous assumption fails to correlate the phenomenon of the moon's acceleration, and the whole subject is consequently as awry as ever.

It is this paganish dogma of conservation of moments that constitutes the chief pillar of the so-called doctrine of

\section{Conservation of Energy}

"The quantum of energy within any isolated system is absolutely invariable in amount".

I peremptorily challenge this teaching as a gross libel on Nature and a. wanton perversion of the plain facts. Laymen, in general, seem to have imbibed the impression that in this generalization science has struck rock-bottom, and that it constitutes a virtual guarantee of the perpetuity of the universe and a solid foundation upon which to anchor the science of physics for all time to come. No conclusion could be farther from the truth. The classical English work on this theme is that of Prof. Balfour Stewart, and was first published in the year 1870 . This is how he concludes:

We are led to look to a beginning in which the particles of matter were in a diffuse chaotic state, but endowed with the power of gravitation, and we are led to look to an end in which the whole universe will be one equally heated inert mass, and from which everything like life or motion or beauty will have utterly gone away.

Although you and I can have no personal concern in the ultimate fate of the physical universe so far as our present existence is concerned, yet I imagine that we all experience a greater or less philosophical interest in the academic question as to whether the universe had a beginning and whether it shall ever have an end. For my part, the doctrine that the universe is a vast machine in the act of running down from an original state of woundup-ness to a futile and inglorious fiasco, is little short of blasphemy. Nor do I believe that I am alone in this sentiment. The perseverance with which all ranks of 
scientists have sought for generations to account for the well-established longevity of the sun leads me to believe that they also sense this conclusion. In a word, the doctrine of conservation is a priori false.

The commonest definition of energy is, "The power that can or does cause a physical change." In their efforts to visualize the operation of this supposititious principle of conservation in the conduct of nature, it has become usual with scientists to liken the imaginary original supply of it to a great reservoir of water advantageously situated on the summit of a high mountain whence it descends by degrees, circulating and meandering through material nature, appearing now in the guise of motion, then as heat, again as magnetism, anon as light, once more as heat, and so on back and forth, to and fro, indiscriminately, until the final end. This descent from higher to lower potential is spoken of as the "degradation of energy", and the end of the whole process is pictured as a dead sea below the level of which energy can drop no further, and where absolute stagnation will prevail. They have given a name to this figurative sea, namely, "Wärmetod", being the German for "heatdeath". For all energy is conceived by them to pass eventually into the single form of heat. Indeed, the relation of heat to motion is supposed to be so well understood and established that their equivalents have even been reduced to tables to make dynamical computations easy. The study itself is called Thermodynamics, and these are its two so-called "laws":

1. For every unit of energy of any kind that disappears a unit of another kind appears.

2. Heat can only pass from a warmer to a colder body.

You will here perceive that between the original strategic position of the store of energy while in the reservoir and its final inert condition in the sea of Wärmetod, there exists an abysmal distinction. In the latter state, all kinds of energy having become resolved into heat 
alone and all bodies having, by construction, arrived at the same neutral level of temperature, it is apparent that Nature has betrayed herself into a stalemate. Though the sum of energy may still exist, in contemplation of theory, it is, by the same theory, recognized as all foredoomed one day to become forever inert and useless. Conservationists themselves distinguish between kinds of energy by calling the higher levels or potentials, as contrasted with those of lower grade, available, and those of the lower, unavailable. That is to say, all energy except that at the very foot of the course is held to be at once available and unavailable-available as to the lower part of the runway, unavailable as to its upper reaches. As the descent is forever going on, they tell us, the quantum of unavailable energy is constantly increasing at the expense of the available. Here, then, in this general sense, as in the special case of gravitation, we are taught that the primordial, the positive, the motive forces of the universe of matter are losing their virtue and that nature is in an irremediable state of decay.

The extremes to which scientists have shown themselves willing to go in the pursuit of this execrable doctrine are all but incredible, extending even so far as to the open and unreserved repudiation of the principle of universal gravitation itself! Let me ask the kind reader to turn now again to the passage previously quoted from Professor Soddy (v. p. 16) before reading further.

In these lines Prof. Soddy talks as impassionedly as if he had a grievance against Gravitation for having too long imposed on scientists as a real thing instead of a mere assemblage of letters. In Mr. Soddy's gospel, position and power are interchangeable terms, while gravity itself is a low impostor that should be excluded from the polite purlieus of scientific language. But if position, as such, is power, then the energy positioned in Wärmetod is not properly characterizable as energy at all; for how can that be energy which can never in the future by any possibility do work; or how can position continue to be energy when, even in this technical sense, it has ceased 
to be? To talk of energy as becoming unavailable for all time is to assert its becoming non-existent absolutely.

There are but three possible hypotheses by which the relation of matter and energy may be explained, namely; first, by construing them as identical; secondly, by regarding them as separate entities, and, thirdly, by supposing energy to be a state of matter, or, in other words, that it is only, the way matter has of manifesting itself to our senses. Of these hypotheses the first was known as the theory of Phlogiston and prevailed all through the lifetime of Newton; the second is that now universally taught in the schools, and the third is my own.

In the narrow limits of current theory the marvelous force of gravitation cannot be made to fit, so the doctrinaires are little by little sophistrizing it altogether out of existence. In their philosophy gravitation is a tether, a brake, a clog. In the field of planetary motions they have even cast doubt upon the actuality of central attractions, because they cannot reconcile it with the secular acceleration of the moon. In thermodynamics they have degraded it into a mere accident of situation. At best, they treat it as a reservoir of power soon drained, instead of as the never-failing well it really is. They say it is not creative; that whatever it is, it will all eventually be converted into heat and perish in stagnation and triviality.

In my philosophy, on the contrary, gravitation is the marvel of all marvels, the immortal fecund mother of all other so-called forces in nature. When gravitation first entered into matter, it made every part of it a permanent magnet, unchangeable, imperishable, and obedient to an unswerving law. Whether a given mass be hot or cold, gaseous, solid or liquid, separate or in combination, its inherent power to attract and be attracted remains forever identically the same. The power of gravitation cannot be crowded into matter, nor can it be abstracted from it-like the widow's cruse, the world of matter preserves its fulness. Gravitation needs not to be fed, nor rested, nor fostered. Every instant it wells up afresh. The at- 
traction that the earth exerts today upon the moon, the sun upon his planets, the stars upon each other, is not the same that will be employed to-morrow or next day; the supply for the future, Nature will evolve as it is required. The attraction of gravitation exercised to-day had no existence yesterday, whether as such, or as heat, or as electricity, or as any other entity. Though always flowing afresh, its supply is not being in the least depleted, nor is Nature being despoiled of anything beyond. Further than this, the force of gravitation is multiple. The earth, for example, attracts the sun no more and no less than it would were the moon out of the way, yet it attracts that body too, to say nothing of all the other celestial bodies-each of them with precisely the same strength as though only the two existed. Again, the power of gravitation makes itself felt over the abysses of space instantly, not, as in the case even of light itself, after a space of seconds or years. Finally, it cannot be eclipsed, as by the interposition of the earth between the sun and the moon. Ponder this marvel! Force emanating out of nothing, uniform, all pervading, instantaneous in action, eternal,- the Hercules condemned forever to perform the labors of the universe; to hold and draw the planets in their orbits; to turn them round their axes; to stoke the suns; to heap the tides; to scatter the rains; yes, even to point the mariner's compass with the magnetism of tidal friction! An unthinking, rigidly-conditioned force, yet not ending in monotony or ruin, as modern science teaches it must, but emerging in order, automatism, perpetuity, and kaleidoscopic change! Has current science made the most of this amazing energy?

Having cast about so long, yet vainly, for an explanation of the persistency of the solar heat, why not patiently consider the merits of gravitation as a last resort? Behold the sun tempestuously ebullient after a service of more than a hundred thousand times the span of human history, when, as Newcomb has said, were it an ordinary body it would have cooled off in a trifle of 4,000 years. Behold Jupiter and Saturn, elder brothers of our 
earth, that, according to the doctrinaires, were set out to cool ten million centuries ago in the absolute zero of space, yet even they, mere dwarfs as compared to the sun, are still glowing with fervid heat. Behold the starry host, whose genesis lies in darkest shadow; yet in the present we see them brilliant and serene without a hint of senility about them. Surely there is a principle of vitality about this universe of ours that the wise have overlooked!

In the commercial field scientists have for years been seeking to devise methods and means for economizing mechanical power. To some extent they have succeeded, but they have invariably discovered that, for all their skill and care, every successive step in the processes of conversion,-from coal to heat, from heat to steam, from steam to mechanical motion, from this motion into electricity, etc.,-there is a leakage which defies fathoming. Yet in spite of their failure to trace these losses and to demonstrate quantitatively the verity of the doctrine of conservation, they ignore these lessons entirely and cling unreasoningly to this their pet delusion. They seem to proceed on the assumption that phenomena must adapt themselves to theory, not theory to the phenomena. Now, a little reflection ought to convince all that in inanimate nature these transformations, which are continually and uninterruptedly occurring, are likewise amenable to just such leakages as these, and that were the doctrine indeed true, the descent from high potential to the lowest should run its course so fast as scarcely to last a year. Given a mountain reservoir with the sluices left wide open, the rush for the sea would be short, swift and ruinous; given a highland brook continuously replenished by the rains, the flow of power will be equable and perennial. The mills of Nature are not driven by an avalanche, but by steady evolvement of power.

Our doctrinaires tell us that there is no such thing as creative energy in nature. They construe the operation of an infinity of years as if it were but a momentary act. They tell us that the earth attracts the moon and holds 
her to her course, but they fail to recognize that the gravitational power which the earth will bring to bear during the next turn of the satellite does not yet exist, but must spring into existence during the ensuing thirty days. Where, I demand, shall it come from? From an accumulated store of energy? If so, how comes it that this store has not already exhausted itself in the eons past? How comes it that there has not been during astronomical history even the slightest evidence of the depletion of this strange power? It ought to be plain to every thinking mind that gravitation is creative in its nature, creative because matter is dynamical in essence.

By some strange quirk the scientists have excogitated the lunacy that when a weight comes to rest upon the ground, gravity is done for, that its energy ceases. They reason thus because they have trained themselves to confound the idea of space with the antithetical idea of power; in fact they actually identify these two diverse things in their processes of ratiocination. Let me ask, what is it that moves the train-the locomotive, or the track? The locomotive, of course. Now what is it that causes the apple to fall from the tree-gravity, or the free space between the twig and the ground? Gravity, of course. Does gravity, then, act on the apple only while the latter is in the state of falling, or did it not also pull on it while it was yet on the tree, and will it not continue to pull upon it forever, after it has found a permanent resting place on the earth? I expect you to answer in the affirmative. We all agree, then, I trust, that the effective cause for the fall that has taken place is not so unany feet of bare space, but gravity, be gravity what it may. Now gravity has this peculiar property, that it waxes with exertion, not wanes; that is to say, its force increases indefinitely with the shortening of the distance. The attraction upon the apple when lying on the ground is therefore stronger than when it still clung to the tree, stronger than when it was in the act of falling, stronger than at any time in the past. Yet the Conservationists say, in effect: 
"Given this force known to be capable of producing a specific mechanical result, then if the same be increased to a maximum and so maintained indefinitely, its effects will altogether cease and disappear, nor will any other sort of mechanical consequences appear in their place!"

Furthermore, Conservationists in their blind zeal confuse the element of force with the element of velocity, and claim, for example, that a mountain resting on its base produces no mechanical effect, for the bare reason that it is not in motion. The formula by which they thus conjure is this:

$$
\mathrm{E}=1 / 2 M V^{2}
$$

in which $\mathrm{M}$ is the mass, $\mathrm{V}$ the velocity, and $\mathrm{E}$ the energy. Obviously, if there be no movement, $V$ becomes zero, and the substitution of this value in the equation, be the mass as big as it may, will reduce the energy to nothing. Nobody denies that gravitation continues to go on drawing; but we are told that, once settled on a permanent base, the weight of an object, whether a mole-hill or a mountain, counts for nought.

Scientists, furthermore, divide available energy into two sorts-potential, or that which stands in reserve against future use, and kinetic, or that which is in the act of doing work, or being converted. In order to get the scientific view authoritatively before the reader let me present this extract from the illuminating essay on $E n$ ergy by Professor John Gall, formerly of Canning College, Lucknow :

A reservoir of water situated on the summit of a hill is more valuable than the same body of water at a low level. This is owing to the store of energy which it possesses in virtue of its position; and as the water descends this energy may be turned to good account in driving machinery. If the reservoir is situated at a lower level than the machinery the water becomes useless as a motive power. A cross-bow when bent, the main-spring of a watch coiled up, a labourer primed for work with a sufficient supply of food, are instances where the energy is due to position.

We have already seen that when a body is projected vertically upwards it possesses kinetic energy in virtue of its motion. As it ascends its velocity and consequently its kinetic energy de- 
creases, but at every point of its ascent it has gained a quantity of potential energy equivalent to the kinetic energy lost, and when the body ceases to rise, the kinetic energy vanishes and the energy becomes wholly potential. In every position of the body the relation expressed by the following equality always holds good:-

Kinetic Energy + Potential Energy = A constant.

When the projected body begins to ascend, its energy is entirely kinetic, because while at the surface of the earth the potential energy of a heavy body is zero. On reaching its highest point the kinetic energy has vanished, and potential energy appears in its place. As the body descends, the energy is again reconverted from potential to kinetic. In positions intermediate between the highest and lowest points the energy consists partly of one kind and partly of the other, and as the body moves from one point to another, a constant transformation of one form of energy into the other is constantly taking place, subject always to the condition that the sum of the two remain constant.

You will perceive from this statement that clearance for fall, not the power that causes the fall, is looked upon as the vital factor. Is not this prima facie absurd? Gravity, it is admitted, pulls the object downward before the fall began, during the fall, and after the fall, but the Conservationists deny to its operation the production of any effect save for the short span of the fall and the brief moment of the settling of the object after. It is a clear case of mistaking identities. Proceeding on this manifestly false tack, our doctrinaires have in all their theorizing assumed the force of gravity to be innocuous and sterile just when, under the letter and spirit of the law of the inverse square, it rises to its maximum! Let us pursue this train of reflection further:

Knowing, as we cannot fail of doing, that it is in the nature of gravity to be sleeplessly active in the control and movement of stars and systems, does it not appeal to your reason that it should be equally persistent and cosmically productive in situations where molar movement is obstructed? Is it good sense to say, or to suppose, that by bringing power and matter in great quantity together, there must ensue an end to activity and that Nature thus stupidly engineers her own defeat? If gravitation can whirl about stellar masses with ease and 
expedition, do you see no logical reason for assuming a priori that, balked in producing molar movements, it may under the wise management of Nature be diverted to the no less vital task of stimulating molecular excitation and thereby generating heat?

Behold there an enormous mountain of a billion-tons weight. Put in its place an enclosed cone of papier maché of like dimensions; what effect, if any, should such substitution exert upon the supporting shelf? According to the interpretation of the Conservationists, there would be no effect whatsoever experienced by the shelf, except, possibly that its natural elasticity might slightly raise its level with the removal of the weight. The real mountain, say they, produces no thermal results so long as it maintains its level and sinks no lower, for it is not the power that counts but the space through which that power acts! It is upon this particular dictum that the Helmholtzian theory of the source of solar heat is founded, and that the future degradation of the luminous stars into cold, dark clinkers is predicated. In thus failing to distinguish between the dynamical potentialities of diversified weights and treating them all as precisely equal, scientists have, in my opinion, been guilty of a grave and incalculably costly blunder.

The spectroscope informs us that a rare few of the nebulæ are self-luminous-they must therefore be hot. How came they so? What more natural than to attribute the phenomenon to the grinding and crushing of the inner substances by their parent stars, not into powder, simply, but into molecules, atoms, electrons-as fine as you will, even to the point of torpedoing themselves into these great glowing mists?

In a previous chapter I pointed out the property of substances in general to explode by percussion or by sheer pressure; leaving in some minds, perhaps, a lurking doubt as to whether the principle of the critical point of gases can come into play unless a very high temperature be first provided. My contention here is, that, even were the cosmic body composed solely of solid, refractory ma- 
terials that had attained a condition of stable compactness, the process of the generation of heat would continuously go on forever. In my conception, hotness is the natural state of matter when under extreme pressure. Compression is neither more nor less than perpetual impact. Let me make this matter certain:

If a pound-weight were to alight on your toes by falling from a height of six feet or so, it would cause you a moment of sharp agony; but supposing no bones to be fractured, the pain would be soon over. Suppose, again, that you were to allow the weight to drop from precisely the same height as before, but this time on the pan of a spring balance (you noting at the same moment the force of the impact as registered on the scale) and that you should then procure a boulder of this greater weight and merely rest it on your toes-would you not suffer in this case, not merely for a passing instant, but for as long as the boulder remained in position? Most certainly! But the Conservationists, were they to argue consistently with their pet dogma, should say that the boulder would pain you only in its first placement, when it was yet in motion; but that afterward, when it had become motionless, it could not hurt any longer, because then it would have no velocity and consequently possess no energy, whether for paining or for generating heat!

Now, while it is quite true that matter does not sense pain as humans do, yet it (or its molecules) experiences in a state of compression certain irritations analogous to pain, just as it does 'when struck a blow. Compression, by forcing the molecules closer together, excites them to reaction, multiplies their collisions, and produces the condition we call heat. Though our mountain may no longer be sinking, yet as long as it remains superposed on its shelf, its basal strata will endure constant strain and develope a perennial supply of warmth, which, despite the natural cooling of the surface, will maintain the average temperature of the mass above that of its surroundings until the end of time. To some minds long accustomed to think otherwise, this statement may sound paradox- 
ical, but the discovery of radium a score of years ago should serve as a lesson to them not to be too skeptical. The phenomena of Nature loudly proclaim its truth-the boiling sun, the lucid stars, the volcanic earth, the fervid Jupiter, Saturn, Uranus and Neptune-or do they not?

Static pressure, I repeat, or, more specifically, gravistatic pressure, is a perpetual generator of cosmic heat and makes for the permanency of the physical universe as we now behold it. Collisions and impacts, on the contrary, breathe of the accidental, the transitory, the insufficient. The tacks that draftsmen use may easily be driven into the board by merely pressing the thumb upon them, but they can just as well be driven in with blows of a hammer. Once driven home, you may either continue the pressing or the pounding, but the tack goes, and can go, no farther. Science says this surplus pressure is not conserved, but that the excess blows are. I deny it.

The force of a hammer blow may be ascertained in terms of dead weight by merely letting fall on the pan of a spring scale and noting the poundage it registers. With such blows as this you may drive in a stake, or beat an anvil, or do many other things you might have in mind to do. If you cared to, you would not need to drive in the stake with the hammer at all, but you could hunt up a boulder exactly the weight of the hammer-blow as indicated on the scale, and this boulder, if merely rested on the top of the stake ought to press it into the ground quite as surely. If instead of driving in the stake, you choose to beat the anvil, you will not displace the latter, but you will heat it. Now, if you please, put the hammer aside and bring the boulder and lay it on the anvil and you will find that the boulder will warm the anvil as much as did the hammer-blows-and continuously. And why should this not be so? For how, I pray, is the anvil going to distinguish between a rapid succession of such blows, divided by no interval, and absolute continuity of the bearing weight? It is no more necessary for gravitation to go the roundabout way of first causing a fall in order to bring about the impact as a means of producing 
heat than it was for Charles Lamb's Chinese to fashion the fagots into a hut before starting the blaze for the roasting of the pig.

It is a customary thing in arguing for the doctrine of the conservation of energy to cite the illustration of raising a dumb-bell and directly letting it drop to the ground, and confining the attention to this simple case. It is explained, that in elevating the bell the lifter expends precisely the same number of units of muscular energy which the bell will develop in thermal units on being let go and striking the ground. "Muscular units expended, are compensated by positional units gained, and positional units lost, in their turn, are compensated by thermal units gained; and so on." But suppose the bell is too heavy for you to so much as budge?

$\mathrm{Ah}$, that is a question which you are supposed to be polite enough never to ask. It seems as plain as can be that when you lift the dumb-bell, you use up a certain amount of muscular energy; but it seems just as evident, too, does it not, that when you pull and pull on an iron stanchion, or try to lift a heavy rail, without effecting the slightest movement in either, you are quite as certainly expending muscular energy? In the case of the dumb-bell our good friends tell us these units of muscular energy are replaced in the shape of kinetic energy by the fall of the bell, thus balancing the dynamical ledger; but they do not volunteer as to how they are compensated in the second instance.

Doubtless you have seen a team of horses straining to overcome a rise in the road and being lashed mercilessly by a brutal driver. Ten minutes pass, perhaps, with no progress made, and the mired wheels stick fast. Now, had all gone well and the horses succeeded without delay or interruption in attaining the top of the hill, your Conservationist would unctuously explain that the muscular energy expended by the animals is fully and accurately compensated by the elevation of the load; but he will have little if anything to say as to what becomes of that same amount of energy where the load remains sta- 
tionary. If he tells you the energy in this second instance is lost, he thereby denies the very principle of conservation itself which he professes to believe and uphold. If, on the other hand, he seeks to claim that the dead resistance to the advance of the wheels produces heat, then he concedes precisely what I contend, namely, that static pressure produces heat. And if static pressure produces heat, then what matters it whether this pressure be occasioned by horseflesh, by steam, by electricity-or by gravity? In short, supposing the horses capable by their pulling strength of sustaining exactly their own weight at the end of a pulley, then were you to kill them and pile their carcasses on top of the stalled wagon, the thermal effects on the ground under the wheels would be exactly the same as in the first instance when they were drawing unavailingly.

Again, suppose, in passing along a walk flanking the precipitous side of a hill, you should notice a loose stone just above your head menacing the safety of passers-by and that you should reach up and gently lower the stone to the level of the walk. In this case, by lowering the stone slowly and by laying it softly on the ground you will have done two things; (1) destroyed so many units of your muscular energy, and (2) destroyed about an equal number of the potential units of the stone-but without, however, adding a single unit of any other sort of energy in substitution. Or, by way of variation, suppose you were to lift the dumb-bell to arm's length, but instead of dropping it or lowering it at once you were to keep holding it aloft until you grew weary, and then to lower it again with the utmost slowness,-could any Stewart or Soddy convince you that the energy units gained by this stunt exactly counterbalance the muscular effort you have put forth?

I emphatically deny the first "law" of conservation, namely, that for every unit of energy of any kind that disappears a unit of another kind appears. It is prima facie absurd, implying as it does that all work done is stored energy. In my philosophy, energy is a gesture 
or manifestation of matter itself, not an intangible something independent of and acting through matter. Nor is energy matter, or matter energy, but matter is energetic. I peremptorily challenge this doctrine of conservation as experimentally false, inasmuch as the finest adjustments of the physicist's laboratory invariably exhibit a steady evanescence of energy, with each successive step of transmutation, impossible to trace or to account for and whose resurrection in a new form rests on no surer foundation than bald inference. I challenge the doctrine, again, as philosophically abhorrent, in that it denies the potential integrity of the universe and peccantly diverts its original capital to current needs.

To illustrate: It is Newton himself who is entitled to the credit of originating the conception that the earth's gravity holds the moon in perpetual leash, and that were this power to run out or forget to act, even for a second, the moon would fly off into space. What, then, can be plainer than that the unique force of gravitation is creative, that it evolves afresh in every new instant of time, and that they who deny the existence of such a source of vitality and recuperation in Nature simply misstate the fact? But Newton confined his attention to only one side of the shield; he considered only the matter of centripetal force, but the centrifugal he left to teleological speculations. Now, a creative force on one side serving as the "action" can be evenly counterbalanced on the other only by a like creative force to serve as the "reaction." The centrifugal force, too, then, must evolve freshly out of Nature, and should it run out instead of the central attraction, the solar system would promptly collapse and crash together. In fine, in order to keep the planets gyrating around the sun-that is to say, merely to make them change their places, without at the same time either adding to or taking away any of their so-called "energy of position"-demands the constant exercise of those two enormous sources of creative energy, namely, the sun's gravity, and the stellar resultant. The present teaching is, that to start a body and to stop 
it require the putting forth of equal amounts of energy, but that the transportation in between requires none. I maintain, in opposition, that change of place of any physical body, even along the level, involves a positive destruction of units of energy (using this term in the conventional sense). In brief, the activities of nature-of translation as well as of warmth-literally consume energy, and to offset this loss, I say, nature must and does evolve it.

Finally, consider the case of an electric crane. Imagine one of these to pick up a ton billet and then immediately to drop it. Here your Conservationist will glibly expound that the electrical energy expended in this act is replaced by an exactly equal number of potential units of energy, and that these latter, in turn, are later replaced by a like number of thermal units when the dropped billet strikes the ground. But now suppose that instead of allowing the burden to drop on the instant, the crane should retain its grip and continue to sustain the load, stationary, in mid-air, for, say, an hour. Query: Would, or would not, this performance absorb more "cur'rent" than the first? Most assuredly it would-more than a thousand times as much. What, then, becomes of this excess energy: how is it replaced? It certainly does not transmute into potential energy, for, by premiss, the weight continues stationary. Nor does it pass to any appreciable extent into heat. The true status of the matter is that the "force" of gravity on the one side of the dynamical equation cancels and destroys a specific quantity of "energy" on the other side. The first "law" of thermodynamics, in this case at least, is therefore contradicted and consequently thrown open to general attack and doubt. Moreover, since energy, so-called, is not necessarily "conserved", the attempted distinction between "force and energy, or, force or energy, to wit, that the former is not conserved, whereas the latter is, falls to the ground. In a word, gravitation is a form of energy. 
In order to show the reader how near scientists have already come to laying hold upon these truths, had it not been for their obscuration by the doctrine of conservation, let me quote two passages from standard works. The first of these is taken from Ganot's Physics, Art. 465, (Ed. of 1877, retained in that of 1910).

If a body be so compressed that its density is increased, its temperature rises according as the volume diminishes. Joule has verified this in the case of water and of oil which were exposed to pressures of 15 to 25 atmospheres. In the case of water at $1.2^{\circ}$ C., increase of pressure caused lowering of temperature, a result which agrees with the fact that water contracts by heat at this temperature. Similarly, when weights are laid on metallic pillars, heat is evolved, and absorbed when they are removed.

The second of the extracts is to be found in Professor Richard A. Proctor's Our Place Among Infinities (pp. 117, 118):

At exceedingly high temperature, much greater pressure, and therefore much greater density, can be attained without liquefaction or solidification. And in considering the effect of pressure on the materials of a solid globe, we must not fall into the mistake of supposing that the strength of such solid materials can protect its substance from compression and its effects. We must extend our conceptions beyond what is familiar to us. We know that any ordinary mass of some strong, heavy solid-as iron, copper, or gold-is not affected by its own weight so as to change in structure to an appreciable extent. The substance of a mass of iron forty or fifty feet high would be the same in structure at the bottom as at the top of the mass; for the strength of the metal would resist any change which the weight of the mass would (otherwise) tend to produce. But if there were a cubical mountain of iron twenty miles high, the lower part would be absolutely plastic under the pressure to which it would be subjected. It would behave in all respects as a fluid, inasmuch that if (for convenience of illustration) we suppose it enclosed within walls made of some imaginary (and impossible) substance which would yield to no pressure, then, if a portion of the wall were removed near the base of the iron mountain, the iron would flow out like water from a hole near the bottom of a cask. The iron would continue to run out in this way, until the mass was reduced several miles in height. In Jupiter's case a mountain of iron of much less height would be similarly plastic in its lowest parts, simply because of the much greater attractive power of Jupiter's mass. Thus we see that the conception of a hollow interior, or of 
any hollow space throughout the planet's globe, is altogether inconsistent with what is known of the constitution of even the strongest materials. $* * *$ The effect of pressure in rendering iron and other metals plastic has been experimentally determined. Cast steel has been made to flow almost like water under pressure.

You may perhaps wonder whether Mr. Proctor, who penned these statements a half century ago, may not have been premature in the making of them, and that later researches (during the greatest investigating age of all time) may have contradicted him. No, he was not mistaken, nor does contemporary science throw the slightest cloud of doubt upon either the facts or the inferences as he gives them. Just as Jupiter was then he still is, and such he has been, according to present scientific opinion, for as long, or even longer, than the age of the earth - say a thousand million of years. Why is he still so hot after a million times as long as (according to the orthodoxical Newcomb), had he been an "ordinary" body, should have been sufficient to cool him down from a solar temperature to a condition such as the moon is in to-day? Jupiter is more than five times as far from the sun as our earth is, consequently he receives only 1-27 as much solar heat, area for area, and the ether that surrounds him is colder than liquid air. Why, I insist again, is he still hot, even to glowing? Shall we follow the example of the Conservationists and lazily sit down and wait for the planet to cool, or shall we not rather seek explanation for the phenomenon in routine natural processes?

By actual experiment physicists have demonstrated again and again that the metal, lead, can be liquefied in the hydraulic press. They interpret this phenomenon in the light of their doctrine of conservation as signifying that in its molten state the metal returns just as many units of heat, and no more, as the mechanical energy devoted to its compression. In their haste and obsession it seems never to have occurred to any of the experimenters to watch and wait to see whether the lead will cool down completely to the temperature of the surround- 
ings. As in the case of the vacuum-tube experiment they blindly prejudge the issue.

But let us reason the thing out in a common-sense way. Suppose the experiment to have taken place up to the point where the lead is liquefied, in the cylinder, and still under the full pressure of the piston. We will now assume, to begin with, the possibility that, given time, the lead will cool off and become solid, notwithstanding the continuance of the pressure. At this latter stage imagine the piston to be unclamped completely and then immediately reclamped with precisely the same effort as in the first instance, what effect will the proceeding have on the lead? Will the latter be liquefied afresh? How so? Surely the second clamping, by merely restoring the former conditions, cannot compress the ingot to less compass than before; and without accomplishing this much, according to general acceptation, the lead must remain in the solid state. Alternatively, suppose the metal in this case should nevertheless become liquefied; then in the name of reason why did it not stay liquid in the first place. Again, if no change at all should occur and the ingot under the second application of the pressure retained its solidity just as it was immediately prior to the moment of unclamping, then all the energy expended in the second act, though by premiss precisely the same as in the first, would be utterly lost, not being compensated by any increase of temperature whatsoever in the lead. Shall we then precipitately conclude that, given a particular mass of lead that has once before been liquefied by a certain intensity of pressure, it can never again be liquefied save by a higher pressure than other lead samples would require; resulting at the end of a series of such steps in a specimen of the metal altogether impervious to compression? Such a conclusion cannot be true, surely, for it would be tantamount to saying that gold is not always gold, lead not always lead, nor iron always iron.

When Proctor, therefore, speaks of a cubical iron mountain being able to liquefy its base, and when the 
world of science looks approvingly on and applauds that statement (which it does), they open our way to a choice of these two deductions; (1) that it is the common trait of all cubical iron mountains twenty miles high to liquefy their bases, or (2) that some do but others do not, depending on whether they have ever gone through the performance before. Of these, the Conservationists throughout their ratiocinations show that they unqualifiedly adopt the second as a kind of basic principle, and accordingly they are looking forward to the day when all of Jupiter's mountains of iron, and of lead, and of other materials will have learned not to liquefy their bases and to stay chill against all pressures.

To my mind the thing could not be more obvious than it is, that a mountain of iron which by its pressure once succeeds in liquefying its base will hold that base in a state of liquefaction indefinitely. When lead assumes the fluid form in the hydraulic press, it does so simply because that is the natural condition for it to take on when under that amount of stress. When either iron or lead is in the fluid state, it means they are hot because Nature has ordained that fluid iron, or fluid lead, and a high temperature shall forever go hand in hand. The bearing of heavy loads continuously oppresses matter as it oppresses the human slave, the latter experiencing pain and weariness, the former evincing its discomfort in "molecular inflammation," as it were, or heat. Jupiter, like the sun and stars, is hot for keeps.

Let it not be inferred, however, that all substances react to gravistatic pressure to an equal extent. They no more do so than they so react to acids, or than asbestos reacts to fire in the same way as does ice. With the first spurious law of conservation out of the way, we can now go on experimenting unprejudicedly with the various substances, resignedly allowing Nature to lead the way and refraining from seeking to dictate to her at every turn. Is there, to your mind-in view of the sun whose light and heat have demonstrably endured for millions upon millions of years with no sign of failing, and in 
view, also, of the countless stars of heaven that so fearlessly defy extinction-anything inherently repugnant or illogical in the suggestion that Nature knows the secret for turning to account the enormous compressional foree of gravitation at her command, just as she knows how to employ that force in maneuvering the stars and planets? Is it any less reasonable to suppose that static pressure continuously warms the sun than to postulate the Nebula to have been "inherently" incandescent, or that his high temperature was generated by the impacts of meteors so intensely frigid as to "freeze the earth in which they imbed themselves", or that he is "getting hotter because he is cooling off", or that radium is the cause, though, admittedly, no trace thereof has ever been discovered in the solar spectrum"?

The hypothesis that heat is an entity independent of matter will not bear the probe of truth. If this were the case, the application of high compression should always produce warmth, whereas the fact is it sometimes produces cold. Indeed, heat itself is sometimes utilized in producing cold-for instance, in the manufacture of artificial ice and in liquefying air. I allude here, however, more especially to the peculiarity of water in being at its greatest density, not at its freezing point, but at about two degrees above it. Experiment shows that the compression of this mineral does not warm it at all, but actually colds it, its temperature depending, not upon the vigor of the compressing power, but upon its own density while under, or not under, compression. Its state of highest density once attained and established, I maintain, will permanently hold the water at a fixed low temperature, provided, of course, that of the surroundings is not greatly altered in the meanwhile. In other words, just as lead under excessive compressions will perpetually maintain a temperature superior to that of the surrounding atmosphere, so will water, under similar compression, maintain a lower.

Here we come face to face with a remarkable provision of Nature that is at the same time an indubitable 
corroboration of this reasoning. We have already called attention to the phenomenon that the earth's temperature increases one degree Fahrenheit for about every 60 feet of depth. At this rate of increase the temperature at 12,480 feet, the average depth of the ocean, should be no less than $212^{\circ}$. In direct contrast with this, deep ocean soundings all over the world have shown that the bottom waters are invariably just a little above zero (that is to say, at the temperature appropriate to water's greatest density). On the one hand, then, we have the earth-body being made warm by compression, threatening the destruction of our planet by the accumulation of central fires; and, on the other, an automatic cooling device for conveying this surplus warmth up through the sea-in its passage serving its own good purpose in tempering the water to the needs of the living creatures that inhabit it. Indeed, were it not for this immense additional source of heat supplied by Mother Earth herself, the oceans would long ago have frozen to a great depth (not solid to the bottom, however, for the reasons shown), and not only would all existing life never have come into being, but it is more than doubtful whether any life whatsoever could have got a start.

From time to time the suggestion is revived to sink a well two or three miles deep for the purpose of tapping the earth's store of internal heat; but nothing has come of it as yet, so far as I am aware. That such a project, if carried out in earnest, would bring with it many surprises, perhaps not all of an agreeable character, is scarcely to be doubted. However, there is an alternative method which to my mind is much more feasible. This is to construct presses of suitable materials capable of bringing to bear the highest possible leverage on, say, a body of lead or iron, imbedded in which should be a coil of pipe made of some stout, refractory material, through which water might be kept circulating. Under these conditions the pressure-liquefied metal would continuously preserve a high temperature and steam would be generated without interruption, according with the capacity 
of the machine, the nature of the materials employed and the intensity of the pressure applied.

With this wonderful and inexhaustible source of energy at command mankind can reckon calmly on the future, and view with equanimity the hitherto terrifying prospect that our present visible supplies of unmined coal will become exhausted within the next two centuries. By pressure coolness, too, can be produced, though not to corresponding extremes, by merely allowing water to cool to its lowest point of density and, while in that state, stoutly confining it against future expansion.In this connection, permit another quotation from Professor Stewart (Conservation of Energy, pp. 118, 119):

It may be shown that if the proposition (of conservation) be true, under certain test conditions we ought to obtain certain results-for instance, if we increase the pressure, we ought to lower the freezing point of water. Well, we make the experiment and find that, in point of fact, the freezing point of water is lowered by increasing the pressure, and we have thus derived an argument in favor of the conservation of energy.

Or again, if the laws of energy are true, it may be shown that whenever a substance contracts when heated, it will become colder instead of hotter by compression. Now, we know that ice-cold water or water just a little above its freezing point, contracts instead of expanding up to $4^{\circ} \mathrm{C}$; and Sir William Thompson has found by experiment that water at this temperature is cooled instead of heated by sudden compression.

Let us scan the claim Mr. Stewart makes that this instance he speaks of is a "proof of conservation." In the first place, it will not be denied by any Conservationist that the work done in turning the compression screw is equatable in terms of units of thermal energy. Suppose, now, that a flame were applied to the container until the water was restored to the temperature it had prior to compression, and the clamp then released. What now would be the state of our dynamies account in profit and loss in terms of units of energy? On the side of profits there would be positively nothing, while the losses would be treble, and include the mechanical energy of clamping, of unclamping, and the heat of the flame-yet the status at the end would be precisely what it was at the outset! 
How much more reasonable to say that when under heavy compression water is naturally cold, and as naturally remains so while the load stays on.

The theory that energy is something apart from matter and that heat is energy, whereas its absence is a negation, does not bear out in practice. If you will take two exactly similar metallic vessels, fill both of them with water and tightly seal them and then place one of them over a hot flame and the other out in the bitter cold, you will learn that both alike will rend themselves. One will burst by gaseous expansion due to heat, the other by the swelling of the water into solid ice; in the former case you must wait until the heat accumulates, in the latter, until the last vestige of it has departed! The result in both cases is the same. Why then do they call heat energy, but coldness not? Again, to produce a quart of liquid air requires the waste of a great deal of heat, but the former, by virtue of its frigidity, will accomplish wonders that heat could never begin to do, or undo. The Conservationists will tell you that the work done by the liquid air is in fact a compensation for the thermal energy expended in its liquefication, but what about the work of condensation on the sun performed by the cold of space? Is the inexhaustible cold of space, also, a "product of thermal expenditure"?

The conductivity of earthy matters is notoriously poor, that of marble, for example, being only about 1-100 that of silver, and brick-earth only about 1-200 of the same. The thickness of a few inches of clay in the walls of a crucible will protect the workmen from a fire within it of two thousand degrees, and more. Now, the temperature of the earth's crust varies at the snail's pace of only one degree for 60 feet of earthy matter. Which, I pray, is the more reasonable inference: that the central heat is oozing out at this amazingly odd rate, or that the temperature gradient is due to the fact of gravistatio heat being generated in situ? Anent this subject, let me quote the testimony of Alfred Russell Wallace (Is Mars Habitable, p. 40): 
In order that the problem may be understood and its importance appreciated, it is necessary to explain the now generally accepted principles as to the causes which determine the temperatures on our earth, and, presumably, on all other planets whose conditions are not wholly unlike ours. The fact of the internal heat of the earth which becomes very perceptible even at the moderate depths reached in mines and deep borings, and in the deepest mines becomes a positive inconvenience, leads many people to suppose that the surface-temperatures of the earth are partly due to this cause. But it is now generally admitted that this is not the case, the reason being that all rocks and soils, in their natural compacted state, are exceedingly bad conductors of heat.

A striking illustration of this is the fact, that a stream of lava often continues to be red-hot at a few feet depth for years after the surface is consolidated, and is hardly any warmer than that of the surrounding land. A still more remarkable case is that of a glacier on the south-east side of the highest cone of Etna underneath a lava stream with an intervening bed of volcanic sand only ten feet thick. This was visited by Sir Charles Lyell in I828, and a second time thirty years later, when he made a very careful examination of the strata, and was quite satisfied that the sand and the lava stream together had actually preserved this mass of ice, which neither the heat of the lava above it at its first outflow, nor the continued heat rising from the great volcano below it, had been able to melt or perceptibly to diminish in thirty years. Another fact that points in the same direction is the existence over the whole floor of the deepest oceans of icecold water, which, originating in the polar seas, owing to its greater density sinks and creeps slowly along the ocean bottom to the depths of the Atlantic and Pacific, and is not perceptibly warmed by the internal heat of the earth.

In boring tunnels through the Alps several independent preliminary surveys are made with infinite care, so that the work may be prosecuted from both ends at once with the certainty of accurately meeting in the middle. What would the chances be of meeting thus fortuitously? Now, we have just such a parallel instance in the case of the earth's heat, for, just about five feet under the surface the temperature is uniform the year round and exactly the mean of that of the atmosphere above. How comes it that scientists have never thought worth while to comment on this amazing coincidence,-that the heat alleged by them to have been implanted in the earth tens 
of millions of years ago, and ever since engaged in escaping, should, just in our day and hour, happen so precisely to match the mean surface temperature, which owes itself to a totally independent cause! What was the temperature a hundred feet below the surface a hundred, a thousand, a million years ago? and, since theoretically so much hotter than now, what should have been the effect upon animal and plant life?

If current theory were really correct, our planet would be a very unsafe one to dwell upon, for a central heat of $300.000^{\circ}$ (as indicated by the temperature gradient) would speedily disrupt it, or, rather, there should now be no earth. According to my theory, the rate of such increase, being dependent on the strength of gravity, must diminish the farther down we get, so that the maximum calculated temperature would not be more than half the amount stated. As a matter of fact, it is far below this figure, because of the extensive internal water-circulation that doubtless exists.

Were the planet ever to increase to a great size, like that of Jupiter, for instance, the intense heat generated would quickly dissipate the present oceans, and in time cause the earth to acquire the same general characteristics as that giant orb.

We thus see that the earth's economy provides what it demands, a running supply of heat, and a uniform one. The extreme secular variation implied by the kinetic theory, as currently understood, or by the radium theory, would have proved just as inevitably fatal to earth-life as a secular variation in the sun's temperature. Nature does not dole out treasured heat, but evolves it as she goes along, and with the same lavishness she displays in all else.

In this process of gravistatic-heat production we have the clue to why the universe did not long ago perish by agglomeration into a single mass, and why it never can do so. Without some adequate dispersive force, constant mutual attraction must prevail in the long run and 
in the end bring about a crash that should leave nothing but a stupendous clinker to mark the tomb of Nature.

It is inferrable that all matter has run the celestial cycle many times, so that the chances are a myriad to one that a given planet has not grown by simple accumulations of primordial dust, but owes its nucleus to a fragment from its own sun or a neighboring star. Or, rather, to fragments, for the stars are unquestionably plastic, so that the future planet gains separate existence in the form of a jet of coarse spray divided into many globules, which separately congeal and afterward cling together as a unit when their mutual attraction triumphs over the unequal dispersive effect of the explosion that begat them. That all planets are spherical, follows from the law of gravitation, provided their parts are sufficiently small or mobile. Were the fragment but an irregular and very rigid block its future shape would depend upon its size alone; one very large would melt with the fervor of its self-generated heat, whereas a small one, such as an asteroid or a meteor, might be and stay any odd shape.

\section{LIFE IN THE UNIVERSE}

One of the labors of the chemist is to synthesize compounds for various uses in the arts, and in the pursuit of this object he is often obliged to try hundreds, perhaps thousands, of combinations and permutations before he attains the particular end he desires; if he attains it at all. Thus far, science has never succeeded in experimentally synthesizing protoplasm, the basis of life, or, at least, protoplasm that actually lived. Speculating with regard to gravitation, we may well ask whether that power which, as we have seen, inspires all the activities of the universe, may not, directly or indirectly, be the secret of life-whether, so to speak, life may not be a sort of transcendental form of it, the "will to live" of Schopenhauer. In the sun, in every star, in every planet, indeed, we see the crucibles in which Nature tries out her materials, combining, dissociating, and recombining them, again and again, endlessly, to work out her evolutionary pur- 
poses. When, finally, the star explodes, its scattered parts, though in the main alike, nevertheless carry severally something unique in their chemical make-up that has its peculiar cosmic value. Is it, then, unreasonable to conceive that some one, or many, of these may fortuitously contain in generous measure all the ingredients of this mysterious protoplasm, and, becoming buried in the planet far enough from the surface to enjoy just the right degree and uniformity of gravistatic temperature requisite for prolonged incubation, in the end germinate life?

The size of a planet determines its character absolutely. If over-large it generates too much gravistatic heat to make life possible on its surface; if too small, it does not generate enough to keep its oceans from freezing, and life is again rendered impossible. Since, however, it is the nature of planets to grow, such as are too small, like Mars and the moon, may hope one day to emulate the earth in fecundity, but the major planets are doomed to perpetual barrenness, although they may once have been viviferous.

A close analysis of these and many other facts, such, for example, as the inclination of the earth's axis, the small eccentricity of its orbit, the nice adjustment of its size "gravistatically" to the work required of it, its favorable distance from the sun, the possible fortuity involved in the origination of life, etc., make it seem not entirely impossible that terrestrial man is the only creature in the universe capable of comprehending and appreciating its stupendous beauty and scope. The thought has its terrors as well as its inspiration.

The evolutionist, the physicist, the geologist, the biologist, may all find in gravistatic heat the explanation of many of their enigmas; such, for example, as the assimilation by Nature of her heterogeneous substances into combinations beneficial to plant and animal life; or the distillation of mineral gases, oils, and the like; or the internal structure of the earth; or the explanation of marine life, and so on. 
Having passed the habitable stage, nothing lies in store for a planet but to grow up into a sun or a star, with a retinue of planets to circle around him. Eventually his Gargantuan appetite for devouring lesser surrounding bodies will create of him a vortex menacing to the integrity of the universe, and Nature in very selfdefense will have to decree his extinction. One day comes the crisis. In an instant his huge bulk bursts asunder and is scattered broadcast, with the speed of light, into surrounding space. Some fragments enter other systems carrying with them more or less gravitational disturbances; but most of his substance remains within his whilom domain in the form of a nondescript nebulous cloud enveloping a hailstorm of meteors and larger fragments that instantly begin the process of equilibristic readjustment to the altered conditions; a process requiring a year of ages, and ending at last in a new system of worlds.

Here among the stars, then, do we find Nature acting consistently with her established order on earth; building only to tear down, bearing children that they may die, creating, so there may not be wanting something to decay. Or shall we not rather reverse the philosophical order and say, that she destroys only that she may improve, that she sacrifices the individual, not wantonly, but for the sake of the well-being, salvation, and evolutional development of the universal Whole?

This destruction of stars is as sure as fate, and seemingly as fortuitous as is physical death among mankind. What star shall be the next to explode is a matter of inscrutable chance, and for this reason the stellar map is so wanting in symmetry. But symmetry never has been, nor ever will be, either a guaranty of permanence nor a consummation devoutly to be wished. That variety is the spice of life is a truth recognized by Nature long before she ever thought to create man. Ruling though she does by the most inexorable of laws, she yet manages to evolve infinite diversity, so that no two objects are ever precisely alike. Planetary systems, 
planets, men, flowers, snowflakes, though faithful types in all things of their respective classes, yet possess individual traits distinguishing them from all others of their kind. 


\section{XIII}

\section{THE PLANETS MARS AND VENUS}

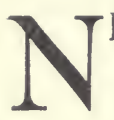

EXT to the earth itself, Mars is by far the most interesting of the primary planets, notwithstanding that it is the second smallest. The reason for this lies partly in the fact that on account of its nearness and its favorable location for observation we can obtain a closer and better view of its surface than we can of any other planet, but mainly in the mystery of its so-called "canals" and the question as to whether it is inhabited by an intelligent race of beings. When very closest to us, Mars is still over $35,000,000$ miles distant, and this distance varies all the way up to $250,000,000$ miles, depending on whether the planet is on the same side of the sun as the earth or not. Inasmuch as the diameter of the planet is only about half (4,200 miles) that of our earth, simple computation will show that, when very nearest, it subtends the same visual angle as a 25-cent piece does at a distance of 700 feet! It should therefore not surprise the reader to learn that there has been a great deal of controversy between first-class observers, armed with the best of instruments, as to whether the "canals" exist at all, not to mention the even more indistinct details which Lowell and others claim to have detected.

The first astronomer to call attention to these lines on the Martian map was the Italian, Giovanni Virginio Schiaparelli (1835-1910), in 1877, who called them canali, which, it is said, does not precisely correspond in meaning with the English word "canals" as subsequently 
employed to designate the same marks. With the closer study of these mysterious lines as his main object, Doctor Percival Lowell (1855-1916) established, in 1894, an observatory of his own at Flagstaff, Arizona, and a year later propounded his now celebrated hypothesis that the canals are the handiwork of a race of intelligent beings who still inhabit the planet and continue utilizing the canals for the purpose for which they were laid, to wit, irrigation.

The fact that Mars is half again as far from the sun as we are, diminishes, of course, the amount of light and heat it receives. This quantity, varying inversely with the square of its distance, amounts to less than fourninths that which the earth enjoys, area for area. Needless to say, the effects of this deprivation of the chief requisites for the preservation of life, assuming its existence, must be very serious indeed, and they constitute one of the chief difficulties to be overcome by the advocates of the Lowellian contention. The surface gravity of the planet, whose mass is but 1-9 of our earth, is reckoned to be 38 per cent as great, so that a rock which on our planet would weigh a hundred pounds would scale but 38 pounds there.

According to Doctor William H. Pickering, who, now that Lowell has passed beyond, is probably esteemed the chief authority on Mars in this country, as he has long been on the moon, in his article in the Americana on the planet describes the canals and other surface features in these words: "They consist of narrow dark lines, generally straight, forming a network over the whole surface of the planet. At their junctions we often find small black dots, known as lakes or oases. Large areas of the planet, called seas, are of a dark gray color, but most of the surface is yellow, or, if observed by daylight, orange. The cause of all the dark regions is probably vegetation, with the exception of the two very black lines which are seen to surround the snow caps when they are melting. These two lines are temporary in their nature, and form the only true oceans of the planet. Occasionally they 
attain a breadth in some places of 300 or 400 miles, and are then found to be of a dark blue color. The polariscope shows that, unlike the rest of Mars, their surfaces are shiny. The yellow regions are thought to be deserts. They cover more than half the entire surface. Very marked changes sometimes appear in the finer details when the snow is melting most rapidly. At the approach of the Martian autumn those parts of the dark areas that are near the poles are seen to fade out and turn yellow so as to be indistinguishable from the planet's soil".

\section{Lowell's Theory of an Inhabited Mars}

Doctor Lowell had long been a close student of Mars and a prolific writer on this his favorite theme, when, in 1909, he published his Mars as the Abode of Life, in which he summarized the whole subject. As basic facts, besides those enumerated above, for his theory, he cites the following (I quote his exact words only in part) :

1. "The northern snow-cap diminishes from $78^{\circ}$ to $6^{\circ}$, the southern dwindles from $96^{\circ}$ to nothing.

2. 'Mars' surface is singularly devoid of irregularities. The more minutely it is viewed the more its levelness grows apparent. Calculation shows that heights even of very moderate elevation should be visible if such existed and none show.

3. "Excluding the polar caps, the surface consists of large robin's-egg-blue patches indiscriminately placed upon a general background of rose ochre, the relative areas being $3 / 8$ ths to $5 / 8$ ths. The tints frequently vary in shade and grade off insensibly into each other, thus making regions of intermediate color but the precise borders of which are not decipherable by the eye". The ochre regions he construes to be deserts of sand and rock intrinsically of that color. "White dots too are scattered over the disk, dazzling diamond points that deck the planet's features to a richness beyond the power of pen- 
cil to portray, so minute are they that good seeing is necessary to disclose them".

4. The fact that the canals appear on the dark blue regions as well as on the ochre leads him to assume that both are land; the former, in his opinion, being probably the basins of evaporated oceans and now covered with vegetation.

5. The canals follow the ares of the planet's great circles, so that we who look centrally down upon them see apparently straight lines. This studious regard for choosing the shortest distance, he opines, implies not only provident economy in design, but also a very high degree of technical knowledge and skill. Necessarily, there are many points of intersection, and, strange to say, at the majority of these there are circular dots about 75 miles in diameter, which in color correspond with the canals. These Mr. Lowell conceives to be oases, Martian cities, as it were, environed by irrigated farms. Not this alone, these canals connect with certain caret-shaped spots that appear to be the "salient points" of the blue-green patches; and from certain of the oases canals branch out numerously, always in straight lines, to other oases, forming a sort of open network. They always lead to definite destinations, never stop short as rivers might do, and, again unlike rivers, they preserve a surprising uniformity of line throughout.

6. In the first days of summer of each hemisphere these canals, he states, begin to grow in distinctness, starting at the polar cap soon after it commences to thaw and thence gradually continuing down to the equator. At such times, too, certain of the canals have a trick of pairing, or doubling.

7. The aggregate length of the canals is enormous, probably as much as 40,000 miles, or three times the circumference of the Martian globe. Five hundred and twenty-two of them have been mapped, the shortest being not less than 250 miles in length and the longest, the Eumenides-Orcus, stretching the enormous distance of 
3450 miles. At least a dozen extend to points far within the polar circles, and would no doubt be found to reach even to the pole itself, were their ends not obscured by the sharp retreating figure of the globe in that high latitude.

After thús carefully setting out his facts, Mr. Lowell proceeds to his theorizing somewhat as follows:

1. According to the molecular theory and the mathematical analysis by Clerk Maxwell, the molecules of hydrogen possess velocities of about seven miles a second, which is slightly higher than the critical, or parabolic, velocity at the earth's surface, and very much higher than this same velocity at the surface of Mars. Now, Doctor Johnstone Stoney has suggested that a logical consequence of this molecular pecularity should be that small bodies like the moon would, because of their feebleness of attraction, gradually lose these swifter-moving molecules and, in the course of years or centuries, become denuded, not only of a large part of their original atmosphere, but more particularly of their original supplies of water, of which mineral hydrogen is an indispensable constitutent. As scientists in general concede this deduction of Doctor Stoney, and as the dead moon is universally believed by them to be devoid of both air and water, or practically so, the inference is that Mars, though more slowly, is following the moon's example and gradually deteriorating into a desert planet at the end of a protracted period of exalted biological development. Mars, then, Mr. Lowell concludes, is an arid planet, and to its inhabitants, if any, water must be at a very high premium.

2. Water is essential to life. There being no oceans, the streams too must be dried up. The alternative is presented of death from thirst, or of recourse to the only fresh water supply remaining, to wit, the polar snows.

3. Naturally this necessity prompted to the Martians the canal idea. Of course the system now in evi- 
dence was not constructed in a day; it was the result, rather, of slow growth, developing painfully in inverse ratio to the failing sources of supply.

4. The undertaking, he opines, was far less formidable than a similar one would be here. First of all, the flatness of the surface would obviate much grading and leveling. Then, again, on account of the lesser surface gravity, the efficiency of the machinery would be perhaps quadrupled. If to these positive advantages be added the suppositions that the Martians are gigantic, and further advanced in the mechanical arts than ourselves, the objection on the score of mere physical difficulty is largely reduced.

5. The surface being flat, every point within as well as beyond the polar circles would be in stable equilibrium. This means that the water would not descend through the canals at any useful speed without artificial propulsion. Observation proves to him that the canals show activity of a certain kind immediately following the incipient thawing of the snow-cap, and that this active movement travels equatorward at the rate of 51 miles a day for a distance of 3300 miles. As to the nature of this change we can judge only from appearance, which consists in a gradual darkening and broadening of the canal lines, and this is construed by him to be due to stimulated vegetation along their borders.

6. The ancient "sea bottoms", he infers from their color, are regions of vegetation. Yet they too exhibit "canals", not only entering from the nearest pole of supply, but rising again into the arid regions nearer the equator. This feat of canalizing the basin of an ocean and thence raising the water to the farther shore $\mathrm{Mr}$. Lowell cites as a striking evidence of Martian engineering prowess.

7. The distance of Mars from the sun, its source of heat, is not so great as absolutely to preclude the existence of animal life. Mars' summer is much longer than ours, and planetary life is determined rather by the mild- 
ness of summer than by the asperity of winter. Mars' mean temperature he estimates at about $48^{\circ} \mathrm{F}$.

8. Unlike the snow caps of our Aretic and Antaretic regions, those of Mars are not permanent, but disappear all but totally every recurring summer season, and are redeposited during the succeeding winter with the same unfailing regularity. Reminding us of the fact that the solar warmth is only 4-9 as great as here, Doctor Lowell argues from these two premises combined that the polar snow deposits must be very scant indeed to be so easily and quickly dispersed.

9. Philosophically speaking, the evolution of life from matter is the order of Nature. Mars, whether or not older than our planet, presumably cooled earlier, both because smaller in size and more remote from the sun. Hence it has already run the gamut of evolution, and, by the principle of survival of the fittest, the present Martians must be the highest products of their race. Historically, they have witnessed the birth of a world and seen it pass through its seven ages into that of the sere and yellow leaf. Even the seas have dried up, partly by absorption into the interior and partly by the escape of the molecules into the outer air. The race is at bay, being driven slowly, but none the less inevitably, to extermination by the road of thirst; for the snow-caps must be thinning year by year, and eventually must altogether vanish never to reappear.

\section{Criticism of Lowell's Theory}

As a scientist, Doctor Lowell was an excellent fictionist. Becoming early enamored of his pet idea, he pursued it relentlessly, in season and out of season, twisting the most intractable of facts to suit his preconceptions; reasoning not to a conclusion, but to support a conclusion previously irrevocably resolved upon. Let us scan his arguments dispassionately with the view of allowing ourselves to be guided by the plain facts rather than by predilection. 
1. First of all, there is the question of temperature -climate. When we say that the sun sheds only 4-9 as much heat on Mars as on our earth, what does the statement mean? By general understanding, Mars, without any solar warmth at all, would possess a surface temperature in the neighborhood of the absolute zero, or $-461^{\circ}$ F. and so would the earth. Even receiving the generous quota of heat we do, the mean annual temperature of New York City is only $53^{\circ}$, of Pittsburgh, $62^{\circ}$, and of Flagstaff, where Lowell himself was stationed, $58^{\circ}$; in other words, the sun's heat, according to common acceptation, serves to raise the superficial temperature of our planet $60^{\circ}+461^{\circ}$, or $521^{\circ} \mathrm{F}$ !! Four-ninths of this number is $232^{\circ}$, which being deducted from $-461^{\circ}$ gives us the exceedingly low average temperature for Mars of $229^{\circ}$ below zero, or $261^{\circ}$ below the freezing point of water! This is surely a long way from the $+48^{\circ} \mathrm{F}$. that Mr. Lowell claims to have derived as the mean temperature for that planet!

2. Vegetation is the food basis of all animal life. Even granting a decline of but $20^{\circ}$ in our mean annual temperature, complicated with the same sort of disastrous fluctuations we have now from week to week, day to day and even hour to hour; what food of value for the sustentation of human life could be grown outside the tropics? And allowing there a reduction of an additional twenty degrees would inevitably put an end to all terrestrial vegetation, save lichens, weeds, grasses, and the like. Yet this reduction would not be more than oneseventh the temperature disparity between the two planets.

3. And did Professor Lowell in his zeal really succeed in persuading himself into the belief that, given sufficient ice-water, vegetation in the arctic zones would proceed as speedily as in the tropics? His own words leave no room for doubt that such is his meaning. For, blindly enamored of his theory, he proceeds, in close logical sequence, to describe how first the coming sun thaws the snow cap, then how the water is made to descend by arti- 
ficial aid toward the equator in the timed flow of 51 miles daily for the huge distance of 3,000 miles, and how, in precise step with this flow, the canals, beginning with the uppermost in latitude, successively darken with the revived growth of vegetation. Dramatically he contrasts the regular poleward trend of our sprouting season with the reversed order on Mars, and seems to derive a discoverer's elation from the circumstances that one is as regular and sequential as the other! Now, there are three conclusive answers to this pretty fancy which Mr. Lowell, for the sake of bolstering up his pet idea, studiously ignores. One is, that unless Mars were much hotter than our earth, the frost in the polar latitudes, where Lowell assumes vegetation to get the earliest start, must be perpetual, and vegetation altogether impossible, whether with or without water, be it warm or cold. The second reason is, that the water freshly melted from the polar drifts would be veritable ice-water, and would be no stimulant to plant-life in a warm, let alone in so frigid a climate; and, third, it is only by screwing up his estimate of Mars' temperature to the last notch that he can convince himself or others that life, even on Mars' equator, is possible, not to speak of canals and oases at its very poles! Then there is the sensible, economical reason, that the canny Martians of the tropics, did they actually exist, should long ago have learned to take thought for the future and to provide stores of water against the opening of the new season, so as not to be dependent upon their Esquimaux for their early spring vegetables!

4. The southern snow cap at the full covers over a quarter of the planet's surface, and the northern cap considerably more than a fifth. The snow must come from evaporation, must it not? But Mr. Lowell denies the existence of any evaporating surface save only of his canals! Were the caps a permanent feature, this point might be overlooked; but the snow disappears every summer, and of course must be as often restored. It seems to me that if Mr. Lowell had not had his fantastic theory in mind, he would have reasoned, sensibly, that the caps 
could not come into existence from the evaporation of his canals alone. The caps being so extensive, and the solar heat so feeble, there must exist an exceptionally large evaporating surface. He might have reasoned further that the vapors from the canals wouldn't extend his theory the courtesy of proceeding intact back to the poles, but would, after the manner of atmospheric humidity, spread in all directions, and come down in rain or snow indiscriminately over the whole surface. His snow eggs are laid without the aid of the mothering ocean.

5. On a planet where warmth is surely as much of a desideratum as water, we should expect to find settlements only in the very warmest belts. The hypersophisticated Martians, however, have proportionately as many canals and oases in the very heart of the arctics as in the best favored regions, and, marvel of marvels, underneath the very snow caps themselves! For proof, consult Mr. Lowell's published maps.

6. Since these canals appear to be feasible everywhere, why did it not occur to the Solons of the planet to surround the snow supply with a belt line, and thus save themselves the manifestly superfluous task of boring a multiplicity of canals through countless miles of what must be hard-frozen soil?

\section{The Author's Views on Mars}

Analogy teaches us to expect seas on Mars, and until better proofs are brought forth than have yet been advanced in rebuttal of this reasonable presumption, we are logically bound to favor it against highly speculative hypotheses such as that of Lowell's. It must be confessed that there is something wonderfully fascinating, to myself as well as to the Lowellians, in the idea that on the planet so near us there may be a race of supermen with whom future earth-born generations may one day hope to communicate, if only by signals; but let us not allow this fancy to cloud our sober judgments. Those of 
us who do not care to surrender the idea may keep on clinging to it if they like, but for the sake of truth and sanity let us not wittingly deceive ourselves with plain delusions.

There is a maxim of law, "Where the reason of the law ceases the law itself ceases," and the spirit of it may well be applied in this place. What purpose are the canals intended to subserve? Lowellians reply, "Irrigation". If it can be shown, then, that there is an ample supply of clouds and moisture on Mars, the motive for building the supposed canal system falls, and the whole theory perishes with it.

When the first edition of this work was in preparation (1911) I sought almost in vain for published testimony supporting my deduction (from my theory of tidal rotation) that Mars is plentifully supplied with oceans; and though more of such testimony may have existed at the time, all I could uncover was what is contained in this paragraph, quoted from that edition:

In one place in his book, Mr. Lowell graphically describes a great Martian storm, in which the cloud was estimated by him to be flying at a height of I4 miles above the planet's surface. He expresses the opinion that this cloud was composed of sand, "because its color was almost that of the planet's surface," which, as we already know, he assumes to be desert. Now if correspondence of color between surface and cloud counts for anything, then the cloud, according to my theory of a frozen-over ocean, should have consisted of snow ; and the issue narrows as to whether sand or snow is the likelier element to be found at an altitude of fourteen miles in a case where the atmosphere is admittdly rare.

Since that year, however, two oppositions of the planet (which occur about two years apart) have taken place, and it has been most carefully studied by a chain of expert observers in various parts of the world led by Prof. W. H. Pickering himself; and by him the results have been elaborately reported, with photographs, in Popular Astronomy, beginning with its January, 1914, number. From these articles I cull only a small part of a great wealth of material to the same effect:

The clouds are sometimes so widespread and numerous, or perhaps so thin, like our cirrus, as to partially conceal the detail 
of a whole hemisphere. This was the case in 1894, and again at one of the more recent oppositions. Photography shows (Harvard Annals 53, 167) that an equatorial belt of cloud is often present, but this must be very thin, since it has not as yet been observed visually. As regards color, it may be noted that our own terrestrial clouds are by no means as white as snow. Sometimes when the sun is low and behind us, two clouds may be seen, the nearer of which is much darker than the other, though it is not possible for either of them to be in shadow. The cause of this seems to be a matter of theoretical interest which has been neglected by our meteorologists.

The more conspicuous Martian clouds lie along the limb of the planet, where their existence can be most readily detected by their irregular distribution. They are also found upon the terminator, especially near opposition, but on account of their wider separation from one another they are not so conspicuous there as upon the limb. Since they are found both at sunset and sunrise, it is believed that cloudy nights are not infrequent upon the planet. On account of its comparatively low pressure, the atmosphere of Mars must at certain seasons contain a larger proportion of water vapor than that of the earth. Some of this would be precipitated at night by the cold, and cloudy nights are therefore what we might naturally expect.

It is believed that the annual circulation of moisture from pole to pole takes place upon Mars as upon the Earth, chiefly by floods and through the atmosphere. Upon the Earth our floods are taken care of, except in a small way, cosmically speaking, by our oceans, but on Mars they must be much more severe. Even upon the Earth, however, where large masses of land occur and the snow fall is heavy, as in Siberia, the spring floods offer a startling demonstration of the intensity of our sunlight. Compared to one of these huge Siberian freshets, our own western floods sink into insignificance.

On January 13, I914, Professor Lowell reported that the Martian southern cap was beginning in stippled patches of hoarfrost, between longitudes $30^{\circ}$ and $60^{\circ}$. Our own next view of this region was on February 7 , when it was occupied by a cloud I 200 miles in length. The cloudy area increased in size the next night to 1800 miles, but two nights later was again smaller measuring 1500 miles. February 12 it had entirely disappeared. The following night all the south polar regions were recorded as very dark, with no trace of cloud. February I4 a crescent of haze lay along the whole length of the limb and part of the terminator, extending half-way to the center of the disk, entirely concealing the polar regions. After this the area in question passed around to the other side of the planet, and we saw no more of it. We are quite clear however that there was no hoarfrost or permanent 
snow area there on either February I2 or I3. The writer does not believe that the hoarfrost is ever seen far from the sunrise terminator, and he would suggest that what Professor Lowell interprets as hoarfrost is described here as cloud. The appearance noted here has a white pearly lustre, clearly fainter than the snow at the north pole, but brighter and much whiter than the desert areas of the planet.

White cloud is occasionally seen in the equatorial regions close to the limb, but when it gets nearer the center of the disk it turns yellow, owing doubtless, as previously stated, to its thinness, and to the fact that we see the soil between separated small clouds. Our own cumulus clouds often have a bluish tint, even near thin centers, when they are forming or dissolving, and are therefore thin enough for us to see our blue sky through them.

It is the writer's impression that the meteorology of Mars resembles very closely that of the earth, much more closely than has heretofore been supposed. Clouds are numerous on both planets but they are more so in the daytime with us, and possess greater density. It is believed for theoretical reasons that the northerly and southerly components of their winds are more marked than ours, that their daily range of temperature is greater, and that their precipitation usually occurs at night, but except in these respects the meteorology of the two planets is practically identical. $* * *$

For many years Martian observers have been in the habit of reporting marked changes as occasionally occurring upon the planet, but the writer believes that a considerable proportion of these, perhaps a third, were due simply to shifting clouds. Thus the numerous and marked changes in size of the southern polar cap at this opposition are almost certainly due to nothing more complex than the formation and dissolution of cloud areas. The sudden appearance of two lakes and four canals in the Protei Regio on December 3I, where on the previous night only a whitish area was visible, was presumably due to the clearing away of clouds. Possibly the fact that certain narrow canals were seen south of the Sabaeus in January, which were not seen on February 14 to 16 , though near the center of the disk, with seeing I2 and ro, is due to their having been hidden by invisible, because partially transparent, cloud masses. (April, I9I4)

When any of the large polar marshes, but especially Acidalium, come around the sunrise limb, they are usually followed at a distance, sometimes as great as 200 miles, by a yellow or white area of considerable size. The former color indicates cloud, and is the commoner of the two. The white probably indicates a thin layer of new fallen snow. It must be thin, since it never persists to the central meridian. On October 9, Martian date February 48, a pure white area one-third the size of the 
polar cap and of the same whiteness and brilliancy, following the Adicalium marsh stretched from the sunrise limb to within $45^{\circ}$ of the central meridian. It must have been an unusually heavy fall for Mars, for this would indicate that it persisted until nearly II o'clock in the Martian morning. Its size was rather difficult to determine for, unlike the snow cap, it had no sharply defined southern boundary. It stretched about 600 miles south from the polar cap, or through some $16^{\circ}$, to latitude $37^{\circ}$, and must have covered nearly a million square miles of surface in the visible hemisphere, and very likely much more on the other side of the planet. (Dec., I9I5).

The snow cap is now appreciably smaller than it was at the last opposition, at the same season of the year. Since as we have already seen it was much larger earlier in the season than at the former opposition, we can hardly doubt but that they have been having rather warmer weather on Mars this year. It will be of interest to note if this means a warmer summer than usual for our Earth.

It may be mentioned here that although we have watched the planet under very satisfactory circumstances throughout the whole of the spring of its northern hemisphere, yet not a trace of green has been detected in that region. The impression gathered has been that all the dark northern areas, and most of the canals observed have been simply marshes, or moistened soil. Possibly greens will appear in their autumn or before. On the other hand the greens of the southern hemisphere, during their autumn, have been at times very striking. They have now all disappeared.

The writer would point out that the clouds always lie over the so-called desert regions of the planet, apparently being precipitated as soon as the fertile regions are reached. This is clearly shown in Figure 14, where the cloud following Charontis is also indicated, half covering Elysium. This was not an unusual feature in this region in the Martian mornings, indicating doubtless that Charontis like Acidalium is composed of moist or marshy land. Incomplete canals, i. e. elongated marshes, disappearing in the desert, are shown in many of the drawings, by most of the observers. Such a thing like an incomplete canal was formerly supposed not to exist. (Dec., I9I6).

Unlike our polar ice caps, which are perpetual, Mars' snow caps totally disappear every recurring summer season, and just as regularly reappear during the ensuing autumn. This circumstance has led Lowell and astronomers in general to suppose that the snow sheet must be exceedingly thin to yield so completely and so readily to the sun's warmth, especially when it is remembered how 
feeble must be the solar rays, not simply at Mars' distance but at his poles. Their error lies in assuming that there is only one way by which snow can be dissolved, namely, by heat; whereas there is this other-BY FLOoDING. Lowell, in words almost emphatic, describes Mars as being remarkably smooth, or level, no roughness of any sort being discoverable on his surface. We have only to surmise, then, that in the frigid season the polar oceans freeze solid and $d r y$, thus supplying a resting place for the snow, and that, during the warm season following, this ice either disintegrates and loses itself, along with its cargoes of snow, in the ocean; or that the ice fields are periodically swept over by tides from the warmer equatorial zones; or that the rains wash down the snow until it turns to slush and so loses its virgin whiteness. Surely, there is no mystery here!

Mars' polar caps are exceptionally extensive, and for their production demand the existence of a relatively large ocean surface. This requirement is rendered more imperative by the sun's remoteness, and is even further accentuated by the compulsory exclusion from the available evaporating surface of that part of it covered, for the time being, by the opposite cap.

If Mars' seas bear a similar ratio to its solid kernel as ours do to the earth's, three-fifths of his surface must be covered to the depth of a mile or more. This assumption, it may be said, is sustained by my theory that the earth's axial rotation is due to the sun's tidal action. Mars rotates on its axis once in $24 \mathrm{hrs} .40 \mathrm{~ms}$., a period reasonably consistent with the earth's case, especially when their respective masses and solar distances are taken into the reckoning.

The center of gravity of Mars (according to my argument regarding the earth) lies toward its north pole, and conformably we can see the outcropping of land there revealed by the persistent presence of $6^{\circ}$ of northern cap, whereas the southern cap seasonally disappears completely.

The conclusion is inevitable: The hitherto supposed "lands", with the possible exception of the blue-green 
patches, must, after all, be seis, and the riddle of the markings must be solved on this basis.

Now, it goes without saying that oceans in their fluid state often exhibit great waves; but these quickly vanish and leave no permanent signs. The insistent reality about Mars, however, is its frigidity, and this fact ought long ago to have prompted the thought that the oceans may be normally frozen over, in which state they can as easily carry surface markings as the most rigid land, and more regular ones, besides.

It would be an error to jump to the conclusion that Mars' oceans must be frozen solid, for decidedly they are not. For one thing, the planet could not rotate unless they were mobile, as I have previously shown; and, for another, the gravistatic heat generated by the planet is probably sufficient to prevent total congelation. Nor should we fail to bear in mind, that the water at the bottom of a deep ocean cannot freeze, because of its density there being constantly maintained above that required for freezing.

That Mars' gravistatic heat is by no means a neglibible quantity may be proved by a simple calculation. Mars' superficial gravity being .38 , one would have to descend toward his center about 150 feet for each degree of increase in temperature, yielding a maximum theoretical temperature at that point of about 40,000 degrees, or an average of 20,000 for the whole mass. This is a much lower temperature than our earth theoretically generates and may seem insufficient until we call to mind that Mars' rarer atmosphere and lesser gravity both operate to greatly lower the freezing point of the water, and that ice is a bad conductor of heat as compared with convection currents in a purely fluid sea.

The excessive external cold must evince itself somehow, and this it does by freezing a thick crust over a major portion of the ocean surface. Nevertheless, even such a surface may experience seasonal changes, and this is the real key to the whole mystery. It remains for us now only to reason by natural steps just what would take place under such conditions. 
Going toward our own north pole we come eventually to a parallel of latitude of perpetual frost. In the case of Mars this critical parallel is nearer the equator than it is with us. During the warm season the ice-crust thaws to the depth of a very few feet, but not entirely through, except, perhaps, very late in the season, and then only right at the equator where the crust is thinnest. So cold must be the planet that the sun would be powerless to cause evaporation were it not for the accidental circumstance mentioned a moment ago that, on account of the diminished surface gravity and lower atmospheric pressure, the boiling point of water there is reduced from our $212^{\circ} \mathrm{F}$. to about $100^{\circ}$, and the thawing point correspondingly.

The process of evaporation takes place from the surface of this glacial crust, or rather from the surface of the thaw-sheet submerging that crust, during the daylight hours of Mars' protracted northern spring of 199 days and his scarcely shorter summer of 183 days. The nights are relatively cold, and during them much snow falls upon the thaw-softened surface, thus giving birth in the course of time to a vast superficial litter of disintegrated ice extending far into the high latitudes.

When this condition reaches a maximum, this superficial sheet of thaw-water is of itself sufficiently deep (or perhaps is sufficiently reinforced by the subjacent waters breaking through the softened crust at or near the equator) to reflect in its movements the tidal effect of the sun. Waves of wide scope are formed, which progress with a high degree of regularity over the smooth, solid ice shelf, sweeping the glacial detritus before them until, having reached the limit of their range, they deposit their cargoes in long, geometrical tidal lines, which then form embryonic ridges, or dams, for future waves and new cargoes to respect and add to ; until in the end the accumulations grow to a width capable of arresting our attention even over this vast abyss of space. Veritable congealed tidal waves are they, following, by the very law of their formation, the great circles of the planet, and supported 
from beneath by the continuous, far-spreading, permanent ice-crust. As the sun moves northward or southward, the new waves that keep forming take slightly different courses, and give rise to new "canals."

Now, when waves touch or cross they "interfere," and create at the points of intersection hills of double height and width. This, I take it, is the origin of the "oases", and of the curious "carets". On this theory it ceases to be mysterious why both canals and oases are so segregated, why so many of them affect the polar regions, and why they connect the "salient points."

The dams, being composed of ice, would melt away were they not seasonally recruited. But they are so recruited, as the gemination process eloquently attests. The seasonal or capricious lightening and darkening of the canals may be aptly explained by fresh snowfalls, or by the meltings of old snows; doubtless as common and natural climatic phenomena there as here, and not especially significant.

The same explanation ought to suffice for similar general variations of color. The brilliant white diamond points which Mr. Lowell describes can assuredly be nothing else than islands capped with snow-his own theory of an exclusively land surface precluding him from advancing the same natural solution. Should any of these ever be detected changing its place, it could be explained as a floating iceberg decked with snow.

My conclusions, then, as to the character of Mars' surface are: That the planet is largely enveloped by its oceans; that these in turn are frozen into great solid, unbroken ice-blocks reposing on their pressure-liquefied bases, and that whatever markings and irregularities may exist on their surfaces, whether comporting with Mr. Lowell's reported observations or not, are the natural result of seasonal changes and tidal wash. Under these conditions the planet is not habitable by any but the very lowest forms of life, and can never in the past have been any better fitted to support life than it is today. 


\section{The Pianet Venus}

We have now seen that the proportion of water, in one or another of its forms, to the solid ball is practically the same in the cases of both Mars and the earth; and we seem therefore constrained to conclude that the same thing is true of Venus. Now, since Venus is almost as large as the earth, its gravistatic heat suffices to preserve its oceans in a fluid state, all the more so from the fact that it receives about twice as much solar heat as does our planet. Venus therefore rotates on its axis. Again, since the planet is so close to the sun, its atmosphere is immensely more humid than ours, and its surface consequently shrouded in perpetual and universal fog; so that we can never hope to obtain even a glimpse of its real face.

If, now, we assume that life is not a happy accident of Nature's, but the inevitable outcome of her inherent energies and laws, we are driven to admit the existence of life on Venus, but not necessarily of intelligent life. Judging from terrestrial analogy it should rather be marine and amphibian in character, on account of the difficulty of lung-breathing; and, moreover, it should be confined to the arctic regions because of what must be the insupportable torridity of Venus's lower latitudes. 


\section{XIV}

\section{THE MOON}

THE general reader would naturally suppose that since we are able to form an idea regarding the

1 character of Mars' surface, which, at the best, is more than 140 times as far from us as is our satellite, it should be a comparatively simple matter to construe the lineaments of the moon. Such, however, has not proved to be the case. In round numbers the moon is only 240,000 miles from us, and has a diameter of 2163 miles, being a fraction over half that of Mars. When viewed through a telescope of about 400 diameters (which is found by experience to be the most satisfactory, everything considered), it is possible to perceive, though as a mere point, any feature upon its surface as large as an ordinary city block.

There are two peculiarities about the moon which have puzzled astronomers beyond measure and which until the initial appearance of this work, in 1912, had escaped their true solution. One of these is that it presents only one side toward us, and the other, that its topography appears to be so abnormally volcanic in character. 


\section{PROBLEM OF THE MOON'S MOTION}

It is truly singular that axial rotation, the cause whereof savants have never yet managed to guess, has been assumed to be the natural thing, whereas a condition of inertness and stability is all their dynamical premises give them the right to expect. So far as we know, Mercury (the smallest of the planets) and the only satellites of other planets susceptible of sufficiently definite telescopic examination (namely, some of Jupiter's) exhibit the same idiosyncrasy of motion as does the moon. It is positively unthinkable that such a uniformity of rotation can be the result of mere chance; but, on the contrary, it must be due, not only to a similarity of causes, but to such causes as inevitably lead to the one result. Astronomers have invariably approached this problem by assuming initial rapid rotations (not attempting to explain them) and thence toning these down, with their imaginary tidal brakes, to fit the observations.

As a matter of fact, the moon doesn't rotate on its axis in any true sense; that is to say, it hasn't an inherent motion of that character, any more than a balloon could be said to have were it also to circumnavigate our globe. Not having any fluid oceans, our satellite has simply settled into a position of stable equilibrium, ballast down, on the familiar principle of the loaded die.

In this attitude the moon makes a circuit about the earth every $27-\mathrm{r} / 2$ days, the plane of its orbit being approximately the same as that of the earth's round the sun, so that we have what are known as lunar phases.

Now, the moon has a peculiar trick, in rounding from full to last quarter, of seeming to turn gently to the east so as to hide a part of that edge and simultaneously expose an equal segment or crescent around the westerly limb. After passing the quarter, the body swings just as gradually backward until at "new", were it then visible, we should see its face precisely as it is at full. In the latter half of its circuit the same maneuver is repeated, except that there we get to see an extra cres- 
cent around the other, or easterly, edge. This phenomenon is what is known as the "longitudinal libration".

In order to solve this peculiarity it will be necessary to mention another fact or two by way of preliminary. The gravitational attraction of the sun on the moon, after all allowances as to distances are made, is a little more than twice that of the earth. Hence it may be asked why the moon does not settle with its center of gravity turned sunward instead of earthward, in which case it would show every side of itself to us, but keep one side perpetually directed toward the sun. The answer to this is not difficult. The test is not one of degree of power alone, but of differentiation of power. For the sun being some 400 times more distant than the earth, the angle formed by two lines drawn from his center to the two points marking respectively the center of figure and the center of gravity of the moon (for the two are by no means identical) will be only 1/400 as great as a similar angle formed by lines so drawn from the earth's center. Hence the sun cannot bring his greater strength so well to bear. It is a question of leverage, or twist. Now, as the moon progresses in her orbit she keeps shifting her angular position relatively to these rival attractions of sun and earth, and this it is that produces the libration in question. Thus, when she is at new, and again at full, the sun and earth act along the same straight line, but when she is at the quarters their attractions conflict most, and the moon swings east or west accordingly.

Here there arises an interesting problem on which we may now gain some light: How far would the moon need to be removed from the earth toward the sun in order to convert her from a satellite of the earth into a primary planet? As matters stand, when the moon passes between the other two, her motion is relatively retrograde with reference to the sun, but direct with reference to the earth. To effect the conversion, then, from satellite into planet, she must exactly reverse this order of motion - a process which obviously would not take effect spasmodically but by infinitely slow degrees, and 
during which she would gradually become more and more distinctly "retrograde" in character. In order for the sun to succeed in "wrenching" the earth and moon apart in this way, his leverage on the moon must, of course, be increased relatively to that of the earth, and as leverage varies inversely with distance, we easily percerve that the moon will become a planet when

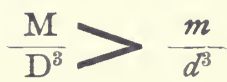

in which M represents the mass of the sun, D his lunar distance; and $m$ and $d$ the mass and lunar distance of our earth respectively. Substituting the known values for $\mathrm{M}, \mathrm{D}$, and $m$ and performing the operation, we obtain for the value of $d, 1,330,000$ miles.

In a former chapter I explained how the earth and moon together are kept revolving around the sun by the action of the Prime Resultant, so that, including it, we have three rival forces continually pulling at the moon, all together being capable of accounting from instant to instant for all her varying movements. If, now, to these were added an inherent arbitrary rotation, as predicated by current theory, it is plain that the moon's safety would be imperilled. Like a weather vane she has to be, and she is, free to respond automatically to the eddying currents continually playing upon her, only in this case the "currents" are gravitational instead of atmospheric in their nature.

\section{PROBLEM OF THE LUNAR SURFACE}

Generally speaking, the moon has a mottled appearance, consisting of great drab splotches on a silver shield. In Galileo's lifetime, and for many years afterward, these splotches were looked upon as seas, and they are still so called by the Latin equivalent, maria. However, they are no longer regarded by scientists in this light, owing to the fact that the improvement of the telescope long ago revealed many permanent markings on the beds of the supposed oceans which, of course, could not exist 
on a liquid surface. In addition to this ocular evidence that the term maria is a misnomer, observers of the satellite unanimously certify that they have never been able to discover any rain clouds in the lunar atmosphere. Indeed, even in this matter of atmosphere the moon appears to be no less destitute, the proof whereof consists in the circumstance that when stars are occulted by her, as occasionally happens, they disappear and reappear suddenly without exhibiting any signs of atmospheric refraction.

This poverty of the moon in water and air may be due to one of four reasons; first, to a scantiness in the original endowment of the body in these two elements, or, second, to the surbsequent escape of their swifter molecules, as suggested by Doctor Stoney, or, third, to percolation and absorption into the moon's interior, or, fourth, to transformation into another state or substance whereby they have become masked and unrecognizable. Of these four hypotheses, it seems, contemporary scientists have finally settled upon the second as the nearest approximation to the truth yet proposed (though still admittedly far from satisfactory) while the last, and, as I hold, the true one, seems never heretofore even to have been thought of, much less discussed.

The most remarkable thing about the strange markings on the lunar ocean beds is not the fact of their existence, nor even the peculiarity of their shapes, but the singular resemblance which they bear to the extraordinary structures that exist in such profusion on the continental areas. The same forces that modeled the first class must have unquestionably modeled the second, The only substantial difference between the two orders is one of scale, those on the continents vastly exceeding the others in height; although there exists this minor distinction, that the configurations occupying the bottoms exhibit more of a ruinous appearance.

Compared to the earth's, the lunar surface is ever so much rougher, and these roughnesses differ so fundamentally in their general character as to compel the con- 
viction that they owe themselves to radically different modeling forces. Let us, if you please, examine some of the principal of these

\section{Characteristic Lunar Surface Features}

Craters. Undoubtedly the most striking of these features is what is known as the craters, a name derived from their likeness to the mouths of terrestrial volcanoes, - a resemblance, however, that is only skin deep, and means nothing. To make out the general contour of these peculiar figures, no higher telescopic power is required than Galileo had available, and the probability is that with his first glance through the instrument he interpreted them as indeed volcanic; and that interpretation is the one which, in spite of later arrivals, commands the largest following to this day. The only other hypothesis that may be said to have gained anything like general acceptance among professional astronomers is that formulated by Professor G. K. Gilbert, in 1892, and known as the Bolide or Impact theory, which holds that the Craters are the pit holes made by the blows of fallen meteorites.

These lunar craters vary incredibly in size, ranging as they do from more than a hundred miles in diameter down to a size hardly perceptible through the best telescopes; indicating clearly that, for aught we can say, they may exist even as narrow as a few feet, or even a few inches; which is what I claim to be actually the case. In the multiplicity of their visible number these craters are no less remarkable, running literally into the thousands; and they are, also, amazingly diversified as well-yet, for all that, there is no mistaking their general kinship. This latter consists in two things, namely, that they all possess a surrounding wall or circumvallation, and, second, that the land craters are bolder in relief and more sharply outlined than their squat brethren on the maria floors. To all intents and purposes, indeed, the maria themselves are indistinguishable from the craters, save in the one particular of size. 
Their individual differences, however, are legion. In some craters the encompassing walls are as much as four or five miles in height, while in others, even of very large ground plan, the rampart is scarcely tall enough to be distinguishable; in some the enclosed plain is as level as a floor, in others it is studded with cones whose peaks rise as high as the rampart itself; in some the wall is practically complete and of fairly uniform height, while in the case of a near neighbor the very reverse may be the case. In those craters where the walls are very tall, they are terraced on the inside, while, in those whose ramparts are low, or of moderate altitude, the inside wall is usually sharply precipitous. There are not a few instances where two, or even several, crater walls impinge, and one appears to mount higher on the ruins of the other or others. On the other hand, there are some isolated craters that have great breaches in their bastions, breaches that cleave clear down to the ground and continue thence as canyons for scores and hundreds of miles through a most rugged country, not following the valleys, mark you, but straight through the highest mountains.

Yet, though all the foregoing enumerated details are severally of great importance, and should be rehearsed by the conscientious student with attentive care, the two most significant, because explicatory, facts about these craters are, first, that the deeper the crater the more does the bottom of its pit extend down below the general level of the surface, much as though some giant had dug out a well and piled the excavated earth around the brim, thereby making the shaft doubly deep; and, second, that, steep as may be the inner wall, the outer invariably slopes gently away to nothing, unless some obviously independent formation rises to obstruct its normal trend.

WHIte RAys. "The most puzzling feature of the surface", says Professor E. W. Brown (Americana), "consists in a series of white rays or streaks which radiate from a few of the principal craters in every direction. In their brightness they mask all other shades of tint on 
the surface and seem to continue their course, sometimes for hundreds of miles, quite independently of the nature of the country they cross. Professor W. H. Pickering, however, who has studied the systems carefully, considers that their actual length has been much exaggerated and believes that the apparent length is due to lines of small craters from which they emerge. The most remarkable system is that starting from the crater Tycho, itself of a brilliant whiteness, and giving the whole region the appearance of a globe cracked by internal pressure-a suggestion made by Nasmyth, who actually cracked a glass globe in this way and obtained a striking resemblance".

Seas. Of these the same writer says, "The so-called seas of the moon are simply portions of the surface darker in color than the average and very much less broken up by craters or mountain ranges. These form the main features of the face seen at full moon. They are crossed by thin lines known as rills or clefts which run in all directions, sometimes straight and unbroken for hundreds of miles, even intersecting ranges of mountains and craters and reappearing on the other side. These rills are generally two and rarely exceed ten miles in width, their depth varying from 100 yards up. A curious feature of a different kind is an absolutely straight cut-the great Alpine valley-some 83 miles long, which crosses a range of mountains and under low magnification looks as if some wandering celestial body had grazed the surface"'.

Mountains. These are described in the classical work of Nasmyth and Carpenter (The Moon, Chap. X) in these words, which I would ask the reader to study carefully in preparation for the explanation presently to be offered:

In turning our attention to these features [mountain ranges, peaks, and hillchains] we are at the outset struck with the paucity on the lunar surface of extensive mountain systems as compared with its richness in respect of crateral formations, and a field of speculation is opened by the recognition of the remarkable con- 
trast which the moon thus presents to the earth, where mountain ranges are the rule and craters like the lunar ones are decidedly exceptional. Another conspicuous but inexplicable fact is that the most important ranges upon the moon occur in the northern half of the visible hemisphere where the craters are fewest and the comparatively featureless districts termed seas are found. The finest range is that named after our Appennines. It extends for about 450 miles and has been estimated to contain 3000 peaks, one of which-Mount Huyghens-attains the altitude of I8,000 feet. * * * Another considerable range is the Alps situated between the Caucasus and the crater Plato. It contains 700 peaked mountains and is remarkable for its immense valley I80 miles long and about 5 broad that cuts it with seeming artificial straightness and that, were it not for the flatness of its bottom, might set one speculating upon the probability of some extraneous body having rushed by the moon at an enormous velocity, gouging the surface tangentially at this point and cutting a channel through the impeding mass of mountains. $* * *$ At first thought it might appear that the great mountain ranges were produced by bodily upthrustings of the crust of the moon by some sub-surface convulsions. But such an explanation could hardly hold in relation to the isolated peaks, for it is difficult, if not impossible, to conceive that these abrupt mountains, almost resembling a sugarloaf in steepness, could have been protruded en masse through a smooth region of the crust. *** We believe they may be regarded as true mountains of exudation, produced by the comparatively gentle oozing of lava from a small orifice and its solidification around it, the vent, however, remaining open and the summit or discharging orifice continually rising with the growth of the mountains.

\section{Lunar Surface Changes}

The reader, I trust, recognizes the impossibility of discussing in detail the multitudinous data on this subject of lunar changes in the short space of a single chapter, and I shall therefore compress what must here be said in the narrowest possible space. To give him, therefore, the most information on this fascinating subject in the fewest and clearest of words, I shall quote,-rather disconnectedly, I regret,--from Professor Pickering's invaluable contributions as they have recently appeared in Popular Astronomy (Nos. 219, 223, 237, 238) to which the interested reader is earnestly referred: 
The writer feels the lack of a generally recognized nomenclature to designate certain features upon the moon, particularly those areas of considerable size which darken with the approach of the lunar summer, that is towards lunar noon, and fade out again in the lunar autumn, that is towards sunset. They correspond to the Martian "seas", but since that term is already in use on the moon for other and much larger objects, he suggests the term "fields", which has the incidental advantage over "canals", etc., of indicating what is really meant, that is plains or slopes covered with some form of vegetation. $* * *$

The barren and unchanging region to the south of the crater will be referred to as the "mare". The brightness of two other regions, the "floor" and the "inner field," has been estimated. The latter refers to that small portion of the inner slope of the crater lying directly between the "source", or point from which the canals originate, and the crater floor. This region changes very notably in shape as the lunation progresses. Between the "field" and the crater lies a bright triangular area which contains several more or less variable canals. Indeed, nearly all of the small dark areas on the moon are variable under high illumination, when well seen, either in shape, or density, or both. Except in the case of a few small spots very near the equator, never has any variation been observed on the moon near the time of sunrise or sunset, that is to say near the terminator. ***

Nearly everyone who looks at the moon through a telescope confines his attention exclusively to these regions, because they furnish striking views, are easily identified, and because what is seen is easy to understand. It is largely due to this fact that the impression that the moon is lifeless has so long maintained its existence, in spite of the facts to the contrary. The only time when the moon is interesting to one who is really used to its surface, is when changes are taking place upon it, and these only occur far from the terminator, that is during the lunar summer time. Then they are conspicuous all over the surface to any who will take the trouble to watch and to study them carefully. **

At colongitude $30^{\circ}$ each of the outer canals fork, but the four branches are very faint. The next day they reach their maximum length of 24 miles from source to terminus. Besides the two main canals others are seen in the immediate vicinity. In the dark region just outside the crater they are seen branching in all directions giving somewhat the effect of a country road map. In places one could scarcely travel three miles without crossing one. They are of all degrees of faintness from that of the two main canals already described, down to the extreme limit of visibility. The narrowest visible, however, can hardly be less than 200 feet in width. 
The lunar atmosphere is very possibly considerably more dense than is generally supposed. We know but little about the refractive powers of very rarefied atmospheres when the mean free path of the molecule is of appreciable length. It is not likely however that its density exceeds a few millimeters, and in that case ice when warmed would pass directly into vapor without passing through the liquid form. However tempting the idea might be, and their appearance certainly suggests it, these canals cannot therefore be irrigating channels either natural or artificial. $* * *$

In summarizing our observations it cannot be impressed too strongly on the reader that neither the dark fields nor the canals owe their blackness to shadow. Shadows are most conspicuous at lunar sunrise and sunset, and are absolutely invisible at full moon. These markings are invisible at sunrise and sunset, and are most conspicuous at full moon. Since the outer canals are wholly invisible at sunrise, though the region is well shown, and the inner ones are similarly invisible at sunset, they cannot be due either to troughs or ridges. The blue black color of the fields and canals can only be due therefore to a discoloration of the surface, which develops alike on slopes and levels in the lunar summer, and is invisible in the spring and autumn. We know of no mineral which acts in this manner, and the only possible explanation therefore seems to be that it is due to a covering of vegetation.

Scarcely any professional astronomers look at the moon now-a-days; it is left wholly to amateurs. Many astronomers write text books on astronomy. New editions are constantly being brought out in which they repeat the well worn statement that the "moon is without air, water or vegetation." It is impossible for a single writer to make headway against such odds. Statements and facts relating to bodies like the moon and Mars, which few astronomers ever look at, make progress but slowly. This is because the text-book astronomers never read them, nor do they believe the observers, if they do. There are too many other things that they must read. ***

The writer first began advocating the existence of vegetation on the moon a little over twenty years ago, and has been writing about it ever since. Other astronomers had suggested it before him, but for the last hundred years the matter has been somewhat in abeyance, the last prominent astronomer who strongly favored it being Sir William Herschel. The writer hopes that in another twenty years the idea will have gained some currency, but alone, without the help of others, it is hopeless to expect to accomplish much. $* * *$ 
In colongitude $55^{\circ} .9$, the whole eastern face (of Pico) is resplendent with freshly formed snow, yet not quite so brilliant as at the higher levels, perhaps because on the lower and steeper slopes small projections of rock more frequently interrupt the bright surface. $* * *$

An interesting phenomenon is exhibited at this colongitude, when spots $a$ and $d$ begin apparently to steam. In a few hours thick clouds of vapor are thrown off, especially from $d$, so that its outlines become very hazy, and quite different from its earlier and later appearance, and from the other spots about it. Its color becomes decidedly bluish, and the fog or mist in a thin transparent stream is swept off across the mare to the south, as indicated in Figure 9. The action becomes less violent about colongitude, $90^{\circ}$, and by $95^{\circ}$ has ceased altogether, as a usual thing, although it was observed on one occasion at the base of $d$ as a very faint haze as late as II $5^{\circ}$. All the spots have occasionally been recorded as slightly hazy at about this time, but none of the others are at all comparable in this respect to $d$. At colongitude $76^{\circ} .8$ the ridges of Teneriffe have been recorded as steaming from end to end. In the meantime neighbouring bright spots were perfectly sharp and distinct. This steaming might be either a case of melting snow or of volcanic activity, the steam condensing into ice crystals and falling as snow. The writer rather favors the latter view, since the other spots do not exhibit it. $* * *$

That certain bright spots such as Linné are always hazy is well known. The peculiarity of Pico $d$ is that it is hazy only at specified seasons on the moon, when other neighboring and similar spots are sharp. $* * *$

Turning now to quite a different type of formation, we find scattered over the moon's surface, but especially in the equatorial regions, a series of small craterlets whose interior walls under all illuminations are of dazzling brilliancy. The floors of the smaller ones are also bright. One of the best known of these is Mosting A, some 5 miles in diameter, 3000 feet deep, and situated in longitude $5^{\circ}$, latitude $-3^{\circ}$. On January 27 , I9I 5 , at colongitude $56^{\circ} .3$ the shadows still showed within the crater, but had entirely cleared the center of the floor, which appeared of a uniform brilliant white, without detail. On March I9, I9I3, 6r ${ }^{\circ} .0$, a minute black dot was detected at the center, of less than half a mile in diameter. It was also observed Feburary I8, I9г3, $66^{\circ} .0$. On January 28 , I9I $5,70^{\circ}$.I, the dot was clearly seen, was about a mile in diameter, and was of the same darkness as the region surrounding the crater. Accompanying the dot, and reaching from it towards the north, three-quarters of the way up to the rim. was a faint dark band one mile in breadth. $* * *$ 
The point of first interest perhaps in this investigation is to find when the spots reach their minimum size. Within the craters, the dark areas appear and disappear at about the same interval before and after midday, which therefore seems to be the time when the snow presents the smallest area. In the case of Linné, Professor Barnard and the writer agreed that the minimum occurred one terrestrial day after the lunar midday. $* * *$ It would certainly be of interest to prepare a series of drawings of the craterlet near Littrow B, and determine when its minimum occurs. In the case of Linné and apparently also of Littrow, the white spot is invisible both at sunrise and sunset. Just why this should be so is not very clear, but it would seem to indicate that the moisture can only escape from the vent about midday, and that towards sunset it all evaporates. Towards noon the evaporation occurs before it can get far from the vent, hence the spot is smaller, although brighter at that time than earlier or later. ***

In the case of the mountains, Pico, Pico B, and Straight Range, most of the white spots grow smaller the longer the sun shines on them. Those on the west side of the mountains, towards the rising sun, are of full brightness when the sun first strikes them. Those on the east do not deposit until the sun has been shining on the region for a day or two. It appears as if the ground some little way beneath the surface must be heated up before the moisture can escape. ***

The writer has sometimes been asked, "What reason is there to believe that there is ice upon the moon?" The answer is: "For the same reason that we believe there is ice upon Mars, because the phenomena observed can be more readily explained that way than any other." Whether the ice is deposited upon the surface, or floats as minute crystals just above it, in the form of surface clouds or fog, is not yet clear, but it is believed it occurs in both forms. Where the boundaries are sharply defined, it lies upon the surface. Where the boundaries are indistinct and hazy, as for instance in the case of Linné, it is still uncertain. In the case of the bright rays surrounding Tycho, it is thought the ice crystals are supported in the lunar atmosphere like those terrestrial cirrus clouds to which we give the name of mare's tails. $* * *$

The question whether water formerly existed upon the moon in larger quantities than at present is usually answered in the affirmative, but generally for wholly erroneous reasons. We often hear it stated that the so-called seas are simply old ocean bottoms, - a most improbable view. A casual examination of the lunar surface shows that they were formed at a period subsequent 
to the creation of the larger craters, since we find numerous of these craters partially ruined and dissolved in them, evidences of fusion being everywhere visible. The seas really owe their existence without doubt to extensive fissure eruptions, such as have occurred in past ages in India and in some of our North-western states. During this period extensive areas of the Moon's crust sank beneath the liquid surface and were dissolved in it, much as often occurs at the present time on a small scale in Kilauea, Hawaii. The bottoms of the great majority of the larger craters were also dissolved or softened at this time, indicating that the crust was thin. After this period was over, a second era of volcanic activity began. The craters produced, however, were much smaller than those of the first period, and indeed are comparable in size to some of our own larger terrestrial craters. They are found chiefly upon the maria and smoother crater floors, and may be recognized by their dark color.

That there never was very much water on the moon's surface is rendered certain by the lack of extensive areas of erosion. Nothing is seen at all comparable in extent to what an outside observer would notice in inspecting the earth under similar circumstances. It is probable that all or nearly all of the large craters were originally furnished with central peaks, but in most cases these were destroyed when the floors were liquefied. Of the peaks which are left, doubtless all at one time, as in the case of our terrestrial volcanoes, gave out large quantities of steam and volcanic gases. On account of the rare lunar atmosphere, due to the small force of gravity, this moisture would have been immediately precipitated as rain or snow, so that the erosion would have been confined to, and concentrated about the vents.

In the lunar craters the only evidences of erosion are in fact found on these central peaks, but so marked is the effect in these places, that only a minority of the smooth cones of large size are left, while in the majority of cases we find that they have been partially cut, and sometimes almost completely worn away. $* * *$

It would seem as if there could be but little doubt that the brightest regions shown in these drawings owe their brilliancy to snow. In order to withstand the evaporation due to a tropical sun, unprotected by any appreciable atmosphere, in latitude $\mathrm{II}^{\circ}$, this snow must be rather deep, even if it is being constantly renewed from the Moon's interior. It must be remembered that none of the bright lines shown can be less, allowing for irradiation, than 50 to Ioo yards in width, and none of the circles much less than half a mile. In a rough and jagged region there would be many small steep areas where snow would not lie, but which would become visible to us only, on account of their small size, 
by a diminution of the general brilliancy of the whole area. We might naturally expect therefore that some snowy areas would appear to be brighter than others. $* * *$

A very casual examination of the Moon will show that under high illumination nearly half of the craters exhibit white patches on portions of their interior slopes. Very little attention has ever been paid to them by selenographers. They are sometimes visible as soon as the Sun rises upon them, but more often they gradually develop under the action of the solar rays. They lie on the slopes most directly exposed to the Sun, and as the Sun moves across the lunar sky, they shift from the eastern to the western side of the craters. In the southern hemisphere they circle round the crater by way of the south, and in the northern by way of the north. Even quite near the equator the circling occurs to a certain extent. The whiteness is frequently due to small irregularly shaped bright areas, which are sometimes related to small craters, but more often they lie on some steeply inclined interior slope. They rarely occur on the outer slopes of the craters.

Let one more quotation suffice, this time from the article of Professor Russell W. Porter, entitled Moonscapes (Pop. Astr., No. 238), which is embellished with three instructive ideal views of the moon that cannot he distinguished from Arctic landscapes, or rather snowscapes. He says:

Our nearest neighbor, the moon, is a case in point. The writer, in viewing her surface through his sixteen inch reflector in the comfort of a closed observing room, has frequently caught himself transported to that body, and, in imagination, viewing her scenery from some crater lip or the vast expanse of one of her sea floors. Having himself spent many years above the Arctic Circle, he was struck by a strange likeness of the moon's general aspect to our own polar regions. The long reaches of the frozen polar ocean, traversed by immense pressure ridges and tidal cracks, the dazzling whiteness and clear cut shadows, the desolation and loneliness - all seemed to find a counterpart in the lunar appearance. The accompanying moonscapes were the natural outcome.

\section{Current Theories Regarding the Lunar Surface}

The Plutonic or Volcanic Theory. Since, in our superlatively practical age, we find astronomers, even those of an agnostic turn, ready to subscribe to such 
superstitions as uncaused motions, self-rotating nebulæ, and the like, it is easy to excuse Galileo and his contemporary sympathizers for accepting, out of hand, the preposterous notion that the so-called craters of the moon are veritably volcanic. But that such an infantile idea has been able to survive the discovery of the mechanical equivalent of heat and command at the present moment the confidence of the great majority of learned scientists, is to me one of the most cryptic of modern mysteries.

It is not a difficult matter to compute what the moon's temperature should have been at the close of the still-credited Laplacian cosmic process, and the calculation has been made thousands of times. Thus, Doctor Lowell, in his Mars as the Abode of Life (p. 23), says, "On the principle that the heat caused by contraction was as the body's mass, this state of things on the surface of vur satellite is unaccountable. The moon should have a surface like a frozen sea, and it shows one that surpasses the earth's in shagginess." His calculation, he reports, gives the satellite's temperature as only $-27^{\circ} \mathrm{F}$. "To point out that any volcanic action could be produced by this quantum of heat", he adds, "is superfluous". All this was long known to astronomers, without, however, in the least influencing their doctrinal teachings. Finally Darwin, with a view to helping matters, came to the rescue with his tidal-evolution theory by which he sought, among other things, to account for the moon's originally high temperature by plucking it bodily from the much more massive, and hence much hotter, earth.

It then came to be taught that the ruggedness of the lunar surface is due to the happy combination of these two factors, namely, first, the possession by a small body of a cosmic temperature appropriate to one 80 times larger, and, second, a coincident reduction in the weight of substances; the former factor supplying the desired augmentation of the eruptive forces behind the volcanic activities, and the latter accounting for the relatively farther distance to which the lighter-weight debris was driven. It was, in fact, somewhat analogous, in their 
minds, to the imaginary transplanting of a strong man from here to the moon, where his power to lift weights would be sextupled.

Struthiously shutting our eyes, as the astronomers here do, to all the ifs and ands by which this ingenious deduction has been arrived at, let us provisionally accept it as true and see how well or ill it fulfills the requirements.

In the first place, there is the planet Mars, which is midway in mass between the earth and moon, yet not $a$ single crater or, in fact, any protuberant irregularity at all has yet been descried upon his surface! Judging from these two bodies, Mars and the moon alone, then, we should naturally conclude that the smaller the cosmic body the more rugged. But proceeding another step higher, we find this rule already violated; for the earth is very much rougher than Mars, yet far less so than our satellite. Now, inasmuch as our Vulcanists are universally agreed in holding that our planet, too, is a shrinking body and that the asperities on her surface are due solely to this cause and the kindred cause of volcanic upheaval, it follows that the only salvation for the Volcanic hypothesis is to establish that the moon, when she gained her supposed separate existence, must have been far hotter than Mars and at least as hot as the parturient earth. In fine, the Vulcanists are logically forced to be Tidal-evolutionists, and their theory consequently rests on Darwin's admittedly "wild speculation impossible of verification".

Secondly, investigation has shown that the earth's temperature rises one degree for every sixty feet of depth, and it is only fair to presume that this rule held relatively true throughout her postulated contracting process. Judging by the existing gradient, the temperature at the earth's center should be about 320,000 degrees F. as against, say, $550^{\circ}$, absolute, near the surface. If, now, we conceive the earth to be divided into 81 concentric strata, all of them of equal mass, the temperature of the outermost should average only that degree marking 
the middle of its thickness, or, by calculation, $1250^{\circ} \mathrm{F}$., abs., while the average temperature for the entire planet should be no less than 80,000 degrees! Now, Darwin's idea was not that the moon was explosively ejected out of the heart of the earth, but that she was flung off from its periphery by centrifugal force. Had the former act been postulated, it would be good logic to say that she bore with her the average temperature of the planet, but inasmuch as she was flung off from the outside, her hotness could not have been greater than that of the crust, the very coldest part of the planet, and theoretically very little above what the satellite should have possessed had she separately agglomerated!

Finally, to cut short a long list of criticisms, it is not true that, even had the moon originally possessed the same temperature as the earth, her volcanic displays would have been more devastating in the ratio of six to one. The solar gravity is 27.6 times that of the earth and 165 times that of the moon, yet he ejects matter at times as far above his photosphere as the moon is from the earth. Anyone who has ever watched quarrymen at work knows that "tamping" is a very important part of the blasting process. A heavier charge lightly tamped will not begin to create as much havoc as a lighter one well tamped. Ramming the charge too tight has ruined many a good gun and robbed its owner of his life. The heavier the weight of the superincumbent materials the longer are the explosions delayed and the severer when they come.

The biggest crater on the earth is said to be that of Haleakla, Hawaii, possessing a diameter of less than seven miles; and there are not more than a half dozen terrestrial craters that could be made out by a lunar observer, though armed with our best telescopic instruments. On the moon, on the contrary, there are literally thousands such, a half dozen of the largest being upwards of a hundred miles in diameter, and thence dwindling to invisible sizes. To account for all these dynamically and adequately, Darwin should have accouched his moon 
from the sun, not from the earth; and she should have been as diminutive as Eros, besides.

The Bolide Theory. By way of an alternative hypothesis, Professor Grove K. Gilbert, whilom president of the Philosophical Society of Washington, in an address before that body in 1892, expounded the notion that the earth may primevally have possessed a ring like that of Saturn, and that the lunar asperities were produced by its collapse. The reader will find an excellent presentation of this view in Popular Astronomy, March, 1917, by Donald Putnam Beard, from which I quote:

OrIGIN. If we conceive the moon as an edifice which had its foundation in a ring or shoal of meteors encompassing the primeval earth, and similar to the giant planet Saturn (the meteoric constitution of whose rings was spectroscopically demonstrated by Keeler in I895), and if we imagine this shoal gravitating together and building up our satellite by accretion, no violence is done the essential principles of Laplace's immortal Nebular Hypothesis. Meteors replace molecules, that is all, as long ago pointed out by the late C. A. Young. The mechanical behaviour of a meteor swarm containing individual masses and endowed with the ordinary velocities of meteors would be precisely similar to a nebulous mass of continuous gas.

The mathematical analysis of the mechanical conception of a Saturnian ring is not in place in a discussion of this nature, but by imparting to the postulated meteors in the swarm orbits not widely variant from that of the moon's, and in a similar direction, their initial velocities at impact were small as compared with those created by the moon alone. Since the course of these moonlets were parts of curved orbits with the moon at their focus, they cannot justly be considered as straight lines. By restricting these meteors to a thin plane ring, and assuming a fairly equable distribution through this plane, the distribution of impact angles deduced by Gilbert yields a curve in which 58 per cent deviate from the vertical less than $20^{\circ}$; 70 per cent less than $30^{\circ}$, while 80 per cent fall within $40^{\circ}$ from the true vertical. To the vertical infalls consequent upon this condition is due the prevalent circularity of the craters and obviates a resort to R. A. Proctor's improbable suggestion of an elastic return to circularity.

Laboratory experiments with a lead disk 5.5 inches in diameter and about 0.5 inches thick as a target, into which .22 caliber bullets of the same material were fired, demonstrate experimentally the effects produced by the impacting moonlets upon the 
moon's surface. Interesting replicas of the moon's crater forms were thus obtained by the writer. $* * *$

OVERLAP. An instance in which a larger crater overlaps and partially obliterates an earlier and smaller formation is shown in Maurolycus, in the roughest portion of the moon. The observed fact that there are comparatively few of these examples is eagerly taken by the volcanic advocates as proof positive that the moon's craters are defunct volcanic formations. But the very paucity of instances, far from proving the truth of the vulcanists' contention, is mutely eloquent in our defense, since the probabilities would be overwhelmingly against the survival of this species of "overlap" crater. Yet this superposition of larger over smaller craters is exemplified by Longomontanus, Maurolycus, Hainzel, Schiller and others.

Sculpture. The peculiar plateau of Wargentin and Phoclydes are striking examples-in more than one sense-of some tremendous lava deluge. The first-named object is a smooth, nearly circular mesa 54 miles across and filled nearly to the level of the lowest point of its rim with solidified lava. That Wargentin does not reign alone in his unique grandeur is proclaimed by the partial filling of Gassendi, Letronne and Hippalus to the north; craters which experienced a community of origin with Wargentin and the neighbouring depressions.

As the result of moonlet impacts in the adjacent maria and the fall of lithic dust from their conflagrations, Boscovich is scarcely to be recognized as a crater, while Julius Caesar and LeMonnier have nearly lost their characters. To the vaporization of the more massive bodies the many "ghost craters" on the moon owe their partial effacement, typified by Fra Mauro, Fracastorius and Cassini. As Doctor See wrote concerning these dim spectres of the desolate lunar Hades: "So far as one can see, only two explanations are tenable: I. The deposit of cosmical dust from the heavens, and from conflagrations arising in the impact of satellites. 2. The partial melting down of the walls by the conflagrations which produced the maria, so that only an outline of the original crater walls can be traced."

The southern boundaries of the great Imbrian lava deluge visioned forth as occurring far down the vista of the ages were determined by Pitatus and Hippalus, while southwestward the onslaught of the impacting planetoid's molten flood attained Posidonius and eastward it lost itself in the Oceanus Procellarum. By this memorable world-wide cataclysm, which at one stroke wrought the Maria Imbrium, Nubium and Humorum and the encricling ramparts known as the Apennine and Caucasus ranges, "were introduced the features necessary to a broad classification of the lunar surface." 
Lunar "Valleys". A veritable "Valley of the Moon" is the Rheita Valley. This is a shallow groove of varying width with a shorter off-shoot on the south end. It runs from the eastern edge of the crater Rheita southwestward more than I85 miles to Rheita $d$; its breadth varies from II to 25 miles, with a maximum depth, according to Beer and Madler, of about I I,000 feet. A long, narrow cleft resembling a rill, starts from near Piccolomini and trends southwestward more than 450 miles to near Metius, which temporarily interrupts it ; but it continues its course beyond that crater and to the right of the Rheita Valley.

However, the Alps Valley, a straight defile traversing the lunar Alps range, is the most interesting of them all, betraying an exceptional character which demands for its origin an exceptional explanation. A trough-like flat-bottomed groove trending east-north-east by west-south-west clean across the Alps range; it is 83 miles long by from three and one-half to six miles in width, and from its positioning bespeaks kinship to the Imbrian deluge, thus uniting the furrow group of the western district with the eastern sculpture system.

BRIGHT RAYS. The system of brilliant rays which radiate from the crater Tycho down the lunar disk, like luminous parallels of longitude, and also the wavy streaks converging upon Copernicus; the lesser systems of Proclus, Kepler and Snellius, are the most enigmatic phenomena of the moon's surface. Those emanating from Tycho extend for vast distances across the lunar disk; in one instance - that of the one crossing the Mare Serenitatis-near 18,000 miles. Straight as the famed canals of the desert planet Mars, they seem not to mind obstructing craters or elevations in their predetermined path. As a contrast, those radiating from Copernicus are branched and wavy and much shorter than the Tychonic phenomenon. ***

Most conspicuous at full moon, under the vertical solar illumination, they seem to be superficial colour-streaks only, and one can be seen on the inner floor of Saussure, near Tycho, and may even be traced up its inner cliffs, like a vein of volcanic trap piercing sedimentary rock-strata on our own planet. This is a treacherous analogy, however, as Mr. R. S. Tozer has pointed out. "The lowest visible stratum on the moon is dark, the configuration of the edges of the light coloured portion showing plainly that the darker portions extend underneath. *** Whence, then, the light coloured lava?

These brilliant rays cannot be inner material extruded from beneath a crust rent by tidal stresses, since an exact restoration of level which would not cast shadows at sunrise or sunset along hundreds of miles would be practically impossible. But the suggestion advanced by Mr. William Wurdemann of Washington, D. C., seems more plausible; viz., that "a meteorite, striking the 
moon with great force, spattered some whitish material in various directions." Furthermore, Professor Gilbert, in the lecture previously adverted to, made the prophetic suggestion that "perhaps the free iron and nickel of meteorites may stand sponsor for free sulphur or phosphorus in moonlets."

When astronomers undertake to theorize, there appears to be no limit to the violence of the assumptions they permit themselves. Because one planet out of eight, Saturn, has a ring, it is taken for granted that the earth had one, and a fantastic hypothesis is straightway built upon the gratuitous idea. No one seems to ask, or care, whether the size of the planet can have any bearing on the matter, or whether the distance and measurements of the ring are conditioned in any way, or whether the sizes of its component particles are possibly limited by a natural law, or how such rings come into existence, or how, in collapsing, they do so upon themselves instead of upon their primary, or how they can collapse at all under the Newtonian traditions. The exterior diameter of Saturn's ring is only 173,000 miles, whereas the diameter of the moon's orbit is 480,000 miles-almost three times as great; nor is there any sign in his ring of any considerable nucleus comparable in magnitude to our moon. Should anyone suggest that the disintegration of such a structure would result in the creation of another Saturnian satellite instead of a meteoric shower upon that planet, he would deservedly be looked upon askance.

About the only characteristic marks on the moon's surface that this impact theory even remotely fits is the simple lunar crater, not all the craters, but only that sort of crater that exhibits a small shallow pit without central cones. It does not explain the depression of the pit floors below the general level of the surface, or why some of the craters biggest in diamter have quite low ramparts and exhibit no inner depressions worth mentioning. It does not explain the inner terraces, nor the non-destruction of the existing central cones, nor the strange coincidence of groups of small craters huddled within the enclosures of some of the larger ones. Nor does it explain the remarkable phenomena of sugar-loaf mountains, the 
stupendousness of the Apennine range, the strange immunity from petty bombardment of the vast areas called maria, the wonderful chain of bluffs hemming in the maria, the several-hundred-miles-long ravines, and the like. Least of all does it account for the thousand and one diurnal variations in the map and color of the moon, of which, thanks to such keen and conscientious observers as Professor Pickering, the recorded evidence is rapidly accumulating-since the first edition of this book appeared.

\section{The Moon's Topography Explained}

Paradoxical as it may sound, the real surface of the moon is as smooth as that of Mars! Equally paradoxical may ring the statement that the lunar oceans have risen from their beds and taken up their permanent abode on the dry land. In plain English, they have disappeared, not into outer space, but into sNow, and the mountains and craters that we see are nothing more nor less than the fantastic sculpturings of one Jack Frost. Like the man in the fable who could not see the woods for the trees, so astronomers have been all along failing to see the lunar oceans because of the heaped-up snow! The text books tell us that snowflakes are so exceedingly porous, and absorb so much air in their creation, that a single inch of rainfall is equivalent to a ten-inch fall of snow. Fancy, if you please, some strange freak of Nature whereby all the waters of our oceans and rivers and lakes should be converted into the "beautiful" and settle upon our continents and islands never to return again as water to its ancient beds, what a wonderful and strange sight our earth would present!

This is precisely what has happened to our moon. This is why her surface is so magnificently sculptured, why we see no rain-clouds or oceans upon her, why her atmosphere has "disappeared"-why, in short, she presents the curious aspect and asperities she does. 
Here you may interpose, "Then how do you account for the fact that the same sort of thing hasn't happened to the earth and Mars?" Easily enough. With respect to the sun, the moon does not rotate on her axis in just the same way as she does with respect to the earth, but turns completely round, so that every point on her surface experiences a day one of our fortnights long followed by a night of equal length. In short, her days and nights are virtually two seasons, summer and winter. This strange arrangement, as you can see, accentuates many fold the contrast between the seasons, and causes us to ruminate how the thing works out. We have only to use our eyes - and brains-to satisfy ourselves.

By analogy, we are bound to presume that the moon possesses, or at one time did possess, oceans in about the same ratio to her mass as the earth, Mars and Venus. It is true this is only a presumption; nevertheless it places the burden of proof on those who assert the opposite. The question then confronts us: What effect should these lunar changes of season naturally have on the waters of her streams and oceans? That these would undergo great evaporation under a steady stream of sunshine two weeks on end goes without saying, even were the moon's gravity as great as the earth's, a fortiori in that her gravity is but 1-6 as strong.

The question then arises: Would the seasons evenly counterbalance each other, so that the summer's warmth would completely counteract and undo the gelid effects of the preceding winter? This question is not one for argument, but for objective fact, and the moon's visibly glacial condition is the answer. We have only to suppose that at the beginning of the process, whenever that may have been, the first winter deposited a greater litter of snow than the ensuing summer succeeded in clearing away, so that a residual sheet of snow-how large is immaterial, seeing that time was not of the essence-remained at the end of the first day (or summer) as the nucleus for later accumulations to come. Thus, day by day, with accelerated growth, the store of snow and ice 
accumulated until all the water was congealed and all that the winters had thereafter to do was to keep up the status by merely making good the day's inroads.

Of course, we cannot see the changes taking place on the dark or hidden side of the satellite, but we can easily judge of them from what we can perceive going on on the areas we do see; and these latter are so easily understandable that a formal interpretation ought scarcely to be required here. However, they are so interesting in themselves that we shall consider some of them, by way of illustration, to show how simply to construe even the most complicated formations are.

Maria. These are really ancient ocean bowls from which their whilom contents have taken flight in the form of white flakes, which, settling on the dry surfaces, have made these their permanent abode. Owing to the geometrical fact that the areas of small spheres are, relatively to their mass, larger than in larger spheres, the oceans of the moon were proportionally shallower, averaging, in fact, only about a half mile in depth. As the levels in these receded because of the snow exodus, naturally the protuberances (of uneven height) on the bottom became successively exposed with lapse of time, creating new islands and new resting places for the flakes. Now, it is self-evident that the nearer the pinnacles of these originally submerged protuberances lay to the surface of the sea, the sooner would they have become exposed and the sooner would the flakes begin building upon them; but it is also true that, unlike on land surfaces, such sub-aqueous irregularities are the exception rather than the rule, hence snow mounds on the sea-beds should not only be lesser in magnitude, but fewer in number.

Inasmuch as, according to our premiss, there was never a let-up to this peculiar process of ocean-robbing, the time eventually arrived when the entire floor became virtually uncovered, presenting in reality a land scene in which every bar and sink-hole showed-save, however, that on all the emerged spots there rested columns of snow, more or less squat, and that the sink-holes were 
normally empty. I say normally empty advisedly, for the reason that during the hot season, as we can see today, a good deal of the snow naturally thaws again and trickles down into the lowest places, forming there black pools which contrast vividly with the scant vestiges of snow that may be found in these recurrently flooded regions. Between the blackness of such pools, however, and driven snow, there are, of course, all gradations of white-though no colors - to be seen. These diverse shades are due to one, or to the combination, of two causes; first, to the soaking of low-lying snow as a result of thawing, and, second, to a seaming and corrugating of other snow surfaces, especially on slopes, by irregular thawing, slipping, and settling; the dense shadows of the ridges in the furrows marring the general brightness.

It would be unreasonable for us to expect to find on our ocean beds, could they be revealed to our eyes, the same perfection and profuse diversity of configurations that our land surfaces exhibit, and the same thing is true of the lunar maria. These tell us just what they are. The "ruined" mounds, craters, and other structures we there see are merely the abortive efforts of the Ice King when his supply of raw material had about given out. Apropos of this, I cannot forbear to quote the lucid impressions of an amateur astronomer, the late Mr. John A. Cook, as set forth in Popular Astronomy (No. 235):

For more than thirty years I have been studying and observing the Lunar surface in an amateurish way, using instruments ranging in size from two to ten inches, and have arrived at conclusions at variance with those, so far as I know, held by the great selenographers, living and dead, regarding the above mentioned features.

We find them scattered about over the floors and shores of the Ocean and seas. When found fully down on the sea floor they often present but the merest trace of a ring. Those on the shore will show that part down in the sea destroyed, while that portion of the crater on higher ground is intact.

Writers speak of them as Ruined walls, Submerged walls, Melted walls, and often as destroyed by some unknown cause.

I have searched the works of Neison, Pickering, Elgar, Lohrmann, Fauth, Nasmyth \& Carpenter, Serviss and a host of other more general works to find if some one would not suggest water 
erosion, and if not why not, to account for their ruined condition, but have failed. The opinion seems to prevail that they have been submerged, inundated by molten matter welling up from the interior burying them. As this would require material enough to spread over between two and three millions of square miles, and to a depth of more than a mile, and as we find no trace of such a lava outflow except in the seas, if there, I find it difficult to accept the theory, even with my great respect for the above named masters of selenography.

Craters. The flatness of Mars, a larger body, indicates that the moon should be essentially fully as flat, if not more so. Such a land surface, we know from daily observation, is ordinarily exceedingly cut up by channels into all sorts of irregular islands and peninsulas, diversified by equally miscellaneous small expanses of water of varying depth. Let us pick out, to begin with, a large lake of considerable depth and possessing a smooth floor, and question ourselves what sort of a snow structure would be likely to result were the water to be set to boiling on the coldest day of an Arctic winter. The rising vapor would, as a matter of course, meeting the icy air, transform itself into snow, and, unless driven far by winds, descend and settle on the nearest land, namely, the margins of the lake, where they would continue to build themselves up as long as there was neighboring water being evaporated. Of course, a good deal of the snow would fall back upon the water, but there it could not lie, unless, indeed, the lake froze over, which, in turn, would mean the cessation of the chain. But let the process continue until the lake went dry, what shall we then have? Surely nothing else but a simple lunar crater-a cavity hollowed out of the earth surrounded by a solid wall of white.

If instead of a single lake, you will imagine another one adjoining it, and much deeper, you will find at the end of the operation one complete wall encircling the secand or deeper lake encroaching upon and "ruining" the wall of the first. Let there be an island or islands in the lakes, and you.will have examples of a peak or peaks, whose thickness and height will depend in large measure 
upon whether they were originally submerged, and if so to what depth.

Just as there may be peaks within craters so there may be craters within craters. The difference consists in this, that whereas the peaks are solid columns mounted on islands from snow supplied by the vapors risen from surrounding pools, the craters are hollow chimneys grounded upon the margins of enclosed wells sunken into the floor of the main basin. That is to say, some pools, both large and small, when baled out, exhibit not only protuberances on their floors, but also pit holes that still remain filled with water, and these sub-pools by going through the same modus operandi as did the original one give birth to sub-craters. In fine, every dent in the moon's surface that normally would be filled with water becomes a crater by the conversion of its contents into snowflakes and the settling of the latter on the brims.

The very largest craters on the moon are, literally, the maria themselves, for here was the chief source of the snow supply. Around their borders on all sides, therefore, stand high snow cliffs, some of them very precipitous; while at other places there are terraces, or perhaps gentle slopes masking the elevated character of the mesas or plateaus at their backs. These differences are due to the varying natural depth of the ocean bed at the shore line. If the offset is abrupt, the gathering water from the summer thaws washes and undermines the base of the glacial cliffs, keeping them sharply trimmed, whereas if there is a natural beach, a different order prevails.

When snow is piled too high, its own weight causes it to collapse or telescope upon itself, resulting in two things; first, the top layer flattens out and overhangs the base, forming a lip, which in time loses its balance and falls, forming a terrace or talus; this event being perhaps followed by another similar avalanche farther on, provided the pile be sufficiently deep; and, secondly, the increased weight finally reaches an extreme limit, where It liquefies and squashes out the undermost layer into the flanking pools. These two processes, major though 
they are, are yet beyond the range of our telescopes to reveal, and rest solely upon deduction. Taken in connection with the continuous repair work being performed by the ever-rising snow, they suffice to keep the lunar structures looking remarkably clean-cut and permanent, a circumstance which explains the absence of signs of "weathering" on the moon.

When the process of glaciation originally began, the lunar atmosphere was, of course, very much denser than it is now, because the snow-that-was-to-be had not yet absorbed it. As a consequence, the meteorological conditions at the outset were much different from now, for whereas the winds doubtless then played a strong role in scattering the snows far and wide over the continental plains, these snows are now quiescently restricted to the immediate neighborhood of the ancient pools, accentuating thus the magnitudes and salient features of the lunar sculpturings, more particularly of the craters, at the expense of the inland prairies.

In elucidating a general principle it is usually much simpler and more satisfactory to choose a concrete example. Let us picture to ourselves, therefore, a circular cistern twelve feet in depth and a hundred feet in diameter. Instead of the walls being vertical, however, let us imagine them sloping downward toward the center of the bottom, but so as to leave a circular flat space, say 25 feet in diameter. Now, for the sake of easy reference, conceive of this slope as cut into four steps, each a yard in height. We shall then have a structure resembling the pit of a Roman amphitheater. Again, let us picture standing on the bottom a number of granite blocks respectively 2, 4, 8 and 10 feet in height, to represent natural protuberances. Let us now fill the cistern to the brim with water, thus submerging all the blocks, and start the natural process of evaporation and snowing, limiting at the same time the snowfall radius to, say, a thousand feet; for we must not overlook the present quiescence of the moon's atmosphere, on account of which the snow spreads but slightly. 
Now, until the water in the cistern has evaporated down to the level of the first tread (beginning from the top), the falling snow cannot gain a footing on the water surface, but must settle only on the brim and a slight distance beyond, its depth gradually decreasing to our prescribed snowfall limit. A yard's depth of water, area for area, being equivalent to ten times that heighth of snow, we shall have gained by the evaporation of the first yard a snow rampart all around the brim, say five feet high, sloping gradually backward to nothing. I have just stated that the snow could settle only on the brim, but this is not precisely true, for we must not forget the tallest of the granite blocks, which for one-third of this time has been exposed. It, too, is capped with snow, say to the depth of two feet.

Now suppose another equal period to elapse, and the cistern's depth reduced to the level of the second tread. This time the brim's cap has increased by another five feet in heighth, and the slope behind it in slightly less proportion, because the rising snow brim has in a measure further blocked the spread of the snow. Again, the first tread having all this time been clear of the water, it, too, is covered, and to a depth of five feet; the tallest granite island has also gained six more feet in height; and the second one of eight feet, having been above water twothirds of the period, disports a snow cap of four feet. Thus the process goes on till we reach the circular area at the bottom. In this area we have finally four islands standing in a shallow pool of water less than a yard deep. But note that the first block uncovered is not the tallest now, for its pinnacle being, let us say, only two feet square, lacks the area necessary to sustain a taller column. As a matter of fact the third block has much the broadest top of any, and though still shorter than either the first or second, must in time, as the result of future processes of evaporation and snowfall, grow to a great height; yet, being itself limited in area also, it can perhaps never reach the altitude of the encircling rampart, which by now, let us say, has risen to the imposing alti- 
tude of possibly thirty feet, and will continue rising, given the necessary supply of snow, until it telescopes upon itself by its own weight. But to return to the moon:

Naturally, the melted water that manages to seep back into the basins must, while the sun is still high, immediately undergo again the process of evaporation. Owing, however, to the frigidity of the planet's quiescent atmosphere, as well as to its own self-built prison walls, the rising mist changes into snow before it escapes from the caverns, in which condition it is, of course, undetectable as vapor. Some of this snow doubtless spreads promiscuously over the planet's surface, but a major share of it remains to restore and heighten the old walls. This piling-up process cannot go on indefinitely without eventually causing the peaks to telescope upon themselves from time to time, partly on account of their own overgorged weight, and partly because of the undermining of their bases by the periodical flooding of the bottoms. This telescoping process gives us the key to the terraced effect so generally observed on the inner sides of the crateral ramparts and supplies the reason for the otherwise surprising precipitousness of their faces. It also explains why the mountains and ramparts automatically preserve a maximum uniformity of height, and why the great ringwalls present their characteristic squashy, convolute appearance.

Mountains. Selenographers have in the past regarded as the most puzzling of all facts about the moon "the presence of the most important mountain ranges in the featureless districts termed seas, where the craters are fewest." By my hypothesis, the mystery becomes a mystery no longer. In the oceans, when full, there could not, of course, have been separate pools; hence no craters. But there were doubtless natural islands. These latter, situated as they were in the very heart of the snow-producing regions (these maria) accumulated the tremendous loads, that now mark them to us as "mountains".

The island of Cuba is 730 miles long, the lunar Appennines 450 miles. Imagine rapid changes of the sea- 
sons to occur on the earth as they do on the moon and that the snows from one winter to another would keep on accumulating until Cuba should be crowded with all the snow it would hold, what a wonderful mountain range would it not make- "in the midst of a featureless plain" -and how like to the lunar Apennines! As for the Valley of the same name, that is merely the vacant trough of the sound that originally separated the island from the mainland.

There would, however, be a great difference between the height of the Cuban range of snow mountains and the 30,000-foot Apennines, inasmuch as the surface gravity here is so much greater. The latter mountains are at their maximum possible height, for, however much they may be snowed upon, they cannot grow taller, for the simple reason that their base becomes liquefied and runs out pari passu. Now, snow is snow wherever it may be, and if piled on the island of Cuba would, other things equal, act as it does on the moon. Other things are not equal, however, particularly in this matter of the surface gravity, as just suggested ; consequently our $\mathrm{Cu}$ ban range could, automatically, never exceed a mile in altitude.

White RAYS, RILLS, etc. Imagine, if you please, an immense marsh 500 miles or so in diameter irregularly cut up into all sorts of small patches of land separated from each other by narrow strips of water of varying depths, with here and there a larger expanse of land bearing small ponds or lakes; and picture to your mind's eye how such a scene would be transformed by such a process of glaciation as I have described. In such a case, soon after nightfall, every natural ledge of land, and every ledge that by the sun's evaporation had become uncovered, would again become decked with fresh snow, the depth varying, of course, according to the controlling circumstances. Indeed, twenty-four hours after the sun had disappeared below the horizon, and perhaps earlier, every square inch of the lunar landscape, every nook and cranny, including even the beds of all the streams, lakes 
and maria, would be covered with the mantle of wbite, and this state of things would continue unmodified all through the long night until close upon dawn.wsowith dawn, however, would begin the great thaw, and the solar artist would occupy himself the ensuing day in etching out the land shapes by, at first, melting the snow where thinnest, which is to say, on the ancient water beds, and subsequently accentuating his artistic effects from hour to hour. In fine, the white rays are neither more noriess than the persistent sdeaper depositsmofi sniw piled on the broaderisand noriginally more ralevatedit and seceptive patches of the marishilandi agrim

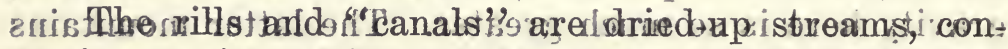

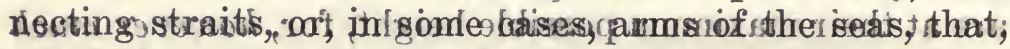
should ithe usnow tall smelt saivayo entiorety, swould fill with waterpto therbring. orille reason whysthey neven fill up with \&nbw isoswelymotehardto understiand, thThough the

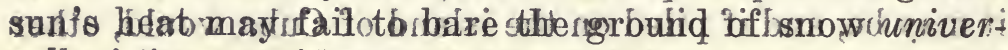
sally, yitiis not said thatuherd wes not thaw grealt quantities of titt a ways sor, evien, st haitr ssomid roff the dand imays not tbe

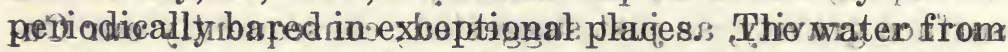

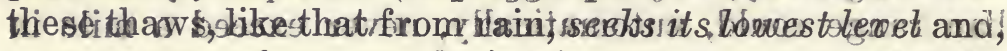
as a matter of course, drains into the ancient channels and pogls. I Arriving there it dissolves what snow may have previouslynittled in thems ior tumbled intor them

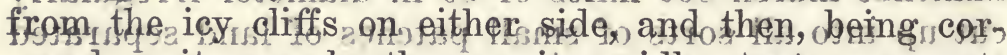

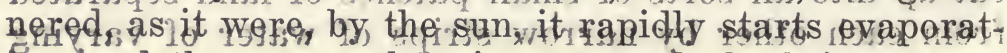

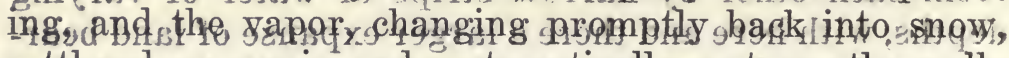
settles dewn again and six tematicaldy westores the walld

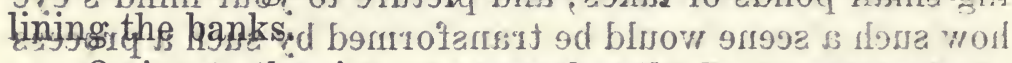

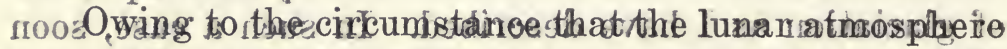
has now found bufialcinbthe [snow:moumods, there lave nos aits

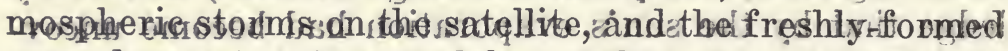

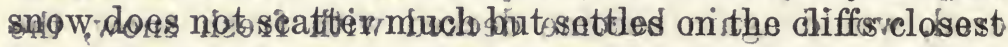

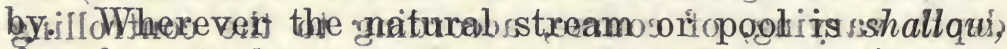
theref $\phi \mathrm{r} \mathrm{e}_{\text {, the }}$ thouer are the adjacent walls, and, converse-

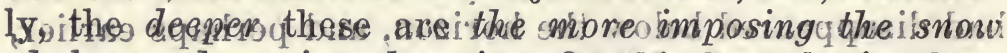

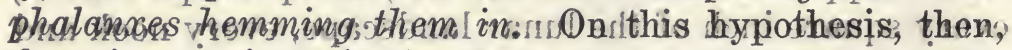

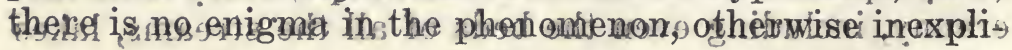


cable; that these supposed "fissures in the lunar crust" cleave straight through the very ruggedest of mountains and abhor the valleys; for this is preeisely what should be expected of them. Nor is there any greater marvel in the observed fact that such clefts and the pits of the deeper eraters actually delve down deep below the natural level of the surface, thereby adding to the "cavernous" .effects' In terrestrial volcanoes the floor of the crater, far from being deeper down than the surrounding regions, is invariably much higher - a most significant distinction.

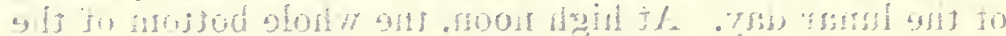

General Reirlemomons.

9\};:1.)

"LUNAR SURFACE CHANGS. The author of all these'is, of course, the sun. At midnight on the moon what little air there is should be absolutely dry, all of its moisture having been frozen out of it and deposited upon the surface in the form of frost and snow. On the deepest beds of the sea this coverfet would be but a mere sprinkle, having fallen after the descent of night as the last act in the drama of the day.

Now, on the moon, owing to the exceedingly light atmospheric pressure, water boils at a much lower temperature than here-let us say at $40^{\circ} \mathrm{F}$., though this can scarcely be more than a close guess. For this reason the thawing process begins early-immediately with, or probably an hour before, dawn-so that (as there is no light refraction there) on the very lowest places such as the mariafloors where it lies at its lightest, the snow may be effectually fooded over before observers can even glimpse its presence. Granting the reasonableness of this inference, it is easy to understand why areas seen to be just whitening as they pass into the night may at their succeeding dawn appear to have lost their whitenes from some nocturnal cause; instead of from the freshets caused by the returning day.

As the sun rises on the lunar landscape, he first shines upon the western sides of the hills; the eastern remaining in shadow, illuminated only by the reflection from the slopes opposites Wherever his rays impinge, 
there the process of thawing goes on and the water of thaw naturally flows down the icy slope or seeps down through the porous snow, until it reaches the pool at the bottom. Once there, the water is warmed to a point of boiling $\left(40^{\circ} \mathrm{F}\right.$., remember, on the moon), and, rising into the thin atmosphere, is quickly reconverted into snow flurries, which to us look to be clouds of vapor and which selenographers have heretofore been construing as "noxious gases from the moon's volcanic vents."

All this, let it be understood, occurs in the morning of the lunar day. At high noon, the whole bottom of the crater, let us say, is directly open to view, and then the pool ought to appear at its largest and blackest, were it not that just then the snow cloud is probably at its densest. With the passing of the meridian, the eastern wall is now being shone upon by the afternoon sun, and inasmuch as the rays start this time with the vertical instead of with a slant, the eastern melting proceeds much more rapidly at the outset, increasing, say, till 48 hours past noon of the lunar day, and thereafter fading to the minimum at sunset. During this half of the process we see the original pool-now in cold shadow-lose its black aspect and freeze over and whiten with a skim of snow, while, diametrically opposite on the crater floor, another pool forms from the draining of thaw water off the eastern slope, giving off fresh clouds of vapor which, being immediately transformed into flakes, settles wherever it can. I use the word immediately advisedly, for Arctic explorers tell us that in those regions the transition of atmospheric vapor into snow is accomplished directly and without the formality of clouds. Commenting on this phenomenon, Prof. W. B. Wright (The Quaternary Ice Age, p. 19) says:

All the explorers who have persisted into the interior (of the Arctic and Antarctic regions) are agreed as to the fine-grained character of the snow which falls there. Among the most interesting of the observations made by Nansen are those bearing on the origin of this snow. He calls attention to the great clearness of the atmosphere during nearly the whole of the time occupied by his journey. On only a few days was the sky overcast, and 
when snow was falling it was still possible to see the sun. The fine dusty snow appears to be precipitated directly from the lower layers of the atmosphere without the preliminary formation of clouds. It is what is known as frost snow in some parts of Norway.

When I use the word "pool", the reader is not to presume that I necessarily mean a circular or sub-circular shape. Its shape may indeed be such, but if so, it would be purely accidental. It all depends on the configuration of the natural water courses, and these, as everyone knows, may be forked and connected up in any sort of way imaginable. Let a deep lake, for instance, be connected with a distant one by means of a channel, then the appearance at noonday would be that of a dumb-bell crater, with a very black handle connecting the two basins. It so happens, in the special case of Aristillus, which Prof. Pickering discusses, that the natural configuration of the crateral basin is such as the dark lines and areas he describes indicate. The basins must possess some shape.

There are, however, it appears, some broader changeful areas in the midst of level plains, far distant from the crateral regions, and upon these Prof. Pickering seems to rely most securely for his contention that there is "vegetation on the moon." My own opinion is that these areas, being far remote from the snow producing pools, are covered with horizontal glacial sheets less than a score of feet in thickness, which either become sopped by the midday thaws into a dull slush or, in places very far remote from the pools, may even be so thin as to melt away entirely and leave considerable spots wholly bare of icy covering. Obviously, under $m y$ theory of lunar conditions, vegetation and animal life there are alike out of the question, and candor compels me to add that, even under the volcanic hypothesis of the origin of the moon's features, which Mr. Pickering champions, I cannot see the reasonableness of his view, best qualified of all men though he may be to speak at first hand on this subject.

Why are there no clouds to be seen on the moon, where so much water exists? My answer is, that vapor 
cannot arise direetly from a crystalline surface, but must

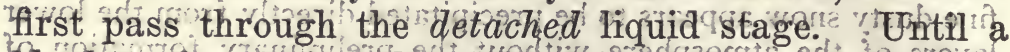
blanket of snow is reduced fo at leasterte condition of a very shallow slush, it will never give off "steam". On the moon there is indeed an immense amount of thawing going on during the day but the fluid water where in itmmediate contact with orystallic ice, preseryes its liquidity and seeps down into and through the porous spongy snow underneath. Not only this, but such little vapor as does form obviously becomes quickly frosted by the "air"' and settles immediately. Either of these explanations alone, or at.least the two acting combinedly, sufficiently explain the anomaly of the moon's cloudlessness as well as the minor fact that the snow peaks are oftentimes perceptibly whiter than their lower, water-soaked slopes.

ALBEDo. Were the surface of the moon perfectly smooth and covered with new-fallen snow, her albedo (i. e., her general brightness) would far surpass the actuality. The deficiency is due to several things, for instance; first, the strong shadows thrown upon her by her own hills, secondly, the slushiness of certain regions, thirdly, the seaming and corrugating of her snow surfaces due to repeated sopping and irregular settling, and, finally, the darkness of her maria. Paradoxically, her dark side is intrinsically whiter than her illuminated face, for then her frozen-over maria and pools are universally covered over by fresh sprinkles of snow.

Absence of Atmospheric Refraction. This peculiarity has heretofore very properly been attributed to a paucity of air; some saying that this was carried off by a passing comet; some that it was probably absorbed by porous volcanic rocks; some that it may have been drawn into the moon's interior by causes unknown; some that its lighter molecules escaped into space, and still others that the satellite never had any more atmosphere than it has just now. As the reader has already learned, my own view is, that it has been almost totally absorbed by union with the water in the manufacture of snow crystals. 
But there is still another reason. When Prof. Langley invented the bolometer about forty years ago and tested it out on the full moon, he was dumbfounded and disconcerted to find that her heat radiation, instead of being much hotter than at new, is really virtually the same. Nevertheless, he courageously reported the fact as he found it, and was severely ridiculed by no less an authority than the great Proctor himself. Since then, the world of science has been trying to wriggle clear of this evidence; which, however, refuses to down. The sun-lit side of the moon, I reassert, is intensely cold, and, being so, the consequent uniformity of her atmospheric temperature from top to bottom greatly diminishes its refractive qualities.

You may wish to ask me why the moon does not rotate, seeing that she was primevally provided with liquid oceans. For this there are two reasons, either one of which would suffice singly. One of these is, that the satellite's gravistatic heat is only a small fraction of the earth's, being in fact only thirty degrees higher at the depth of two miles than it is at, say, 100 feet below her surface. The second reason is, that inasmuch as the lunar oceans could never have exceeded a half mile in depth on the average, and since the weight of water there is only one-sixth of what it has here, the load on the bottom waters could not have been more than fifteen atmospheres and consequently fell far short of the amount requisite to compress them beyond their freezing density. 

Notations 
$2501+-$ 






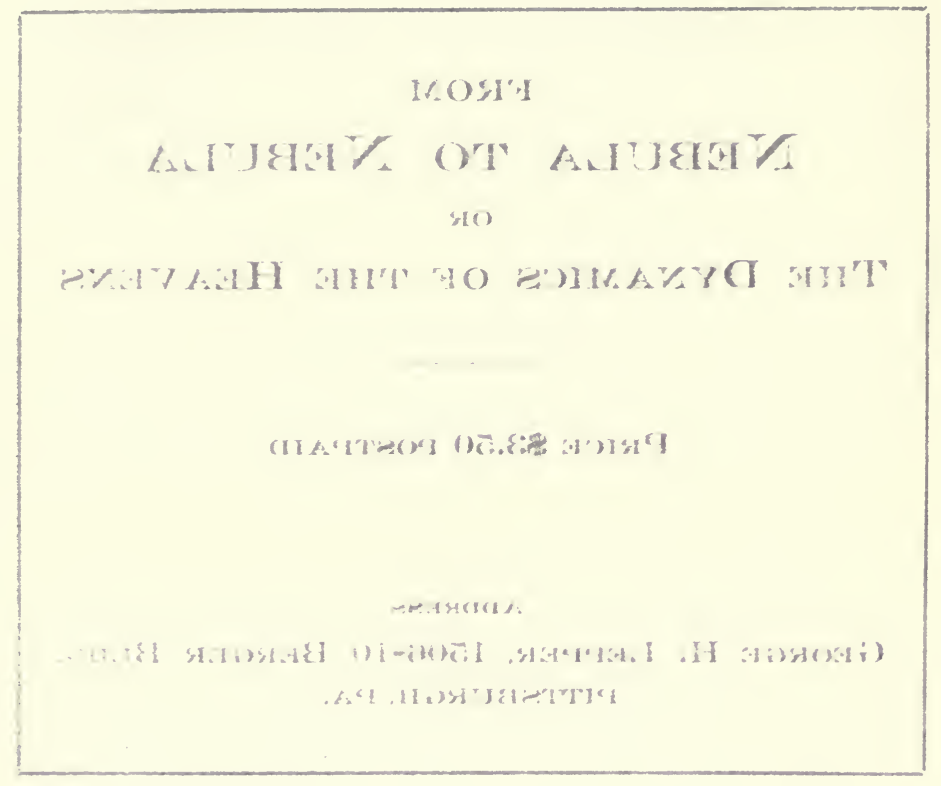




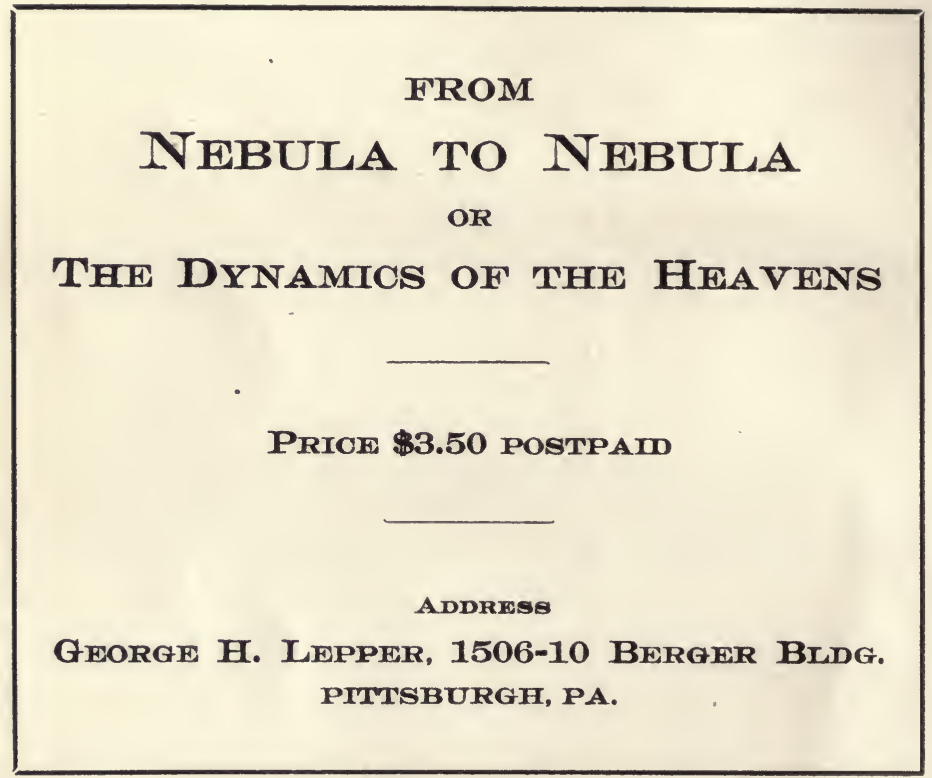


THIS BOOK IS DUE ON THE LAST DATE STAMPED BELOW

AN INITIAL FINE OF 25 CENTS WILL BE ASSESSED FOR FRILURE TO RETURN THIS BOOK ON THE DATE DUE. THE PENALTY WILL INCREASE TO SO CENTS ON THE FOURTH DAY AND TO \$1.00 ON THE SEVENTH DAY OVERDUE.

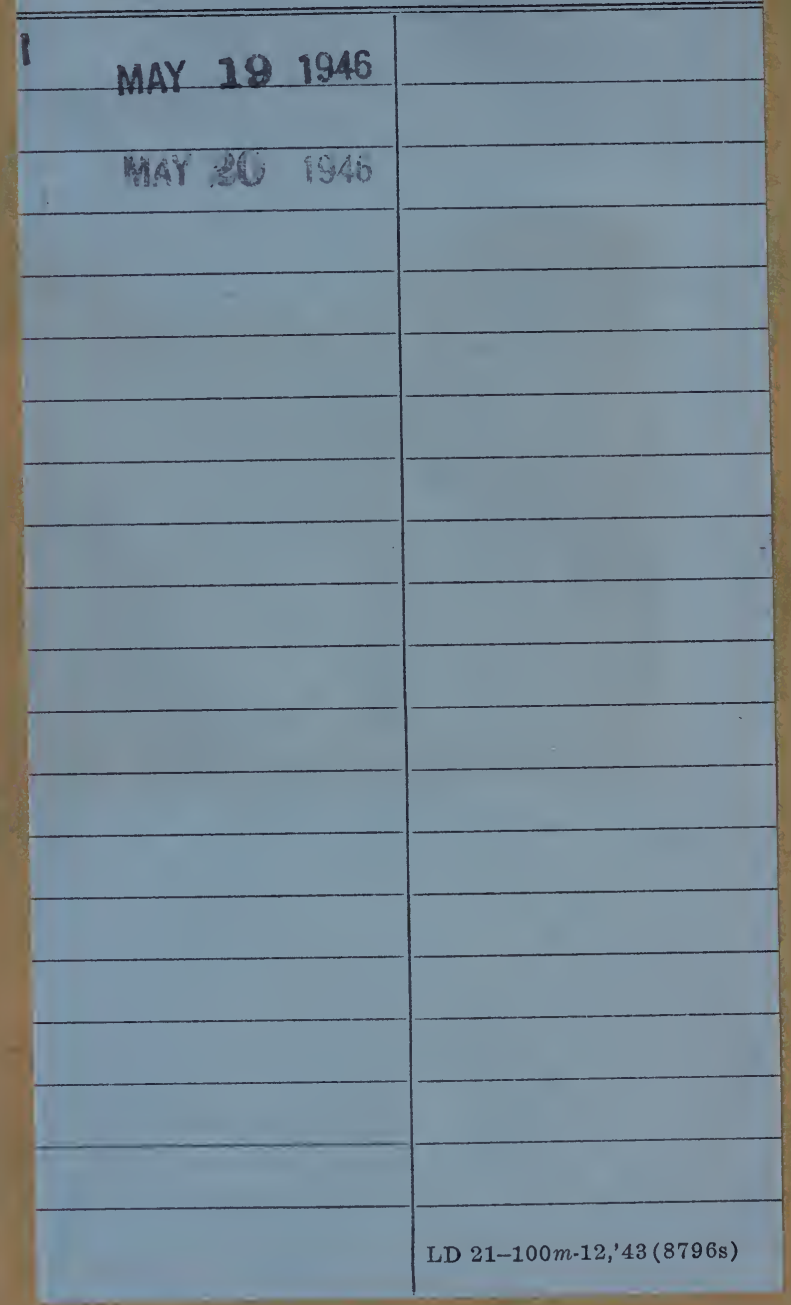


YC 22470

,

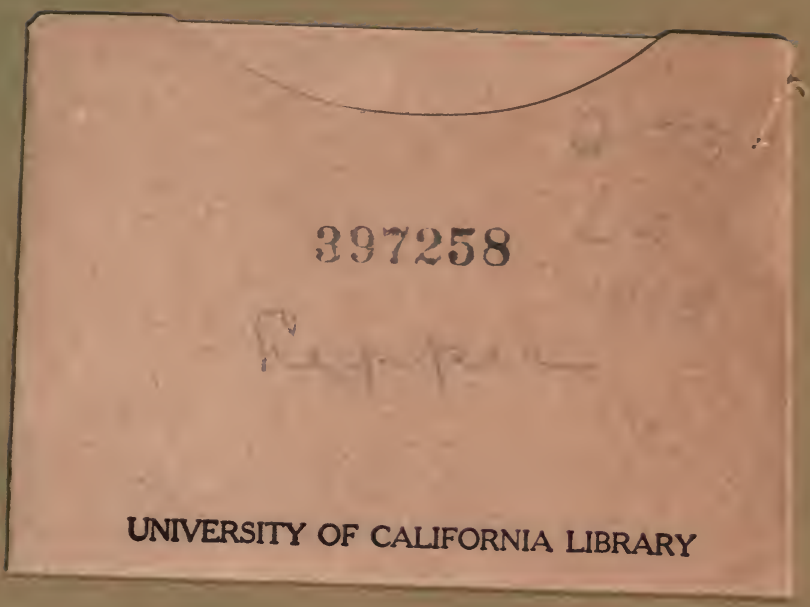


